\title{
The Effect of Ionising Radiation on the Luminescence Properties of Fluoroperovskites
}

\author{
Joseph Schuyt
}

\author{
A thesis \\ submitted to Victoria University of Wellington \\ in fulfilment of the requirements for the degree of \\ Doctor of Philosophy \\ in Physics
}

Victoria University of Wellington 



\section{Acknowledgements}

This thesis contains work performed primarily at Victoria University of Wellington over the past three years. The completion of this work was made possible through the support of several individuals and organisations well-deserving of acknowledgement.

I would firstly express my immense gratitude to my supervisor Dr. Grant Williams, for offering me an honours project that developed into this $\mathrm{PhD}$ thesis, and for his invaluable guidance, support, contributions and discussions over the years. I would similarly express my gratitude to my secondary supervisor Dr. Shen Chong for his assistance and encouragement in completing this work.

I would like to thank Dr. Andy Edgar for introducing me to a range of experimental equipment and techniques and for his sound scientific advice. I would also like to thank all past and present members of the Radiation Imaging and Detection Group for laying the foundations on which this work was built.

The development of the 2D dosimeter system would not have been possible without Don Peat of i2M Labs and his engineering expertise and advice. I also thank those at Callaghan Innovation that assisted with sample syntheses and characterisations, namely Dr. David Clarke, Dr. Sebastiampillai Raymond, and Dr. Robert Breukers.

This project received funding from the New Zealand Ministry of Business, Innovation and Employment, The MacDiarmid Institute for Advanced Materials and Nanotechnology, and Victoria University of Wellington, for which I am very grateful.

Finally, some personal thanks are in order. To my fantastic family, of whom there are far too many to name, for their endless support. To my wonderful friends, old and new, for providing both encouragement and distraction when needed. And lastly, thanks to Maria and India, the most inspirational people I know. 



\begin{abstract}
The luminescence of crystalline compounds can be used to monitor many physical phenomena, including doses of ionising radiation. Optically stimulated luminescence (OSL), thermoluminescence (TL), and radiophotoluminescence (RPL) have been successfully employed in dosimetry. However, few materials possess both the structural and luminescence properties required for medical dosimetry. This thesis aimed to investigate the luminescence features of the class of compounds known as fluoroperovskites. Emphasis was placed on studying the effects of irradiation on the luminescence properties, such that the compounds could be evaluated regarding potential applications in clinical dosimetry. Samples were primarily characterised using photoluminescence (PL), radioluminescence (RL), OSL, RPL, TL, and transmittance spectroscopy.

OSL was observed in the majority of samples due to the existence of electron trapping F-type centres. F-centre/Mn complexes were observed in all $\mathrm{AMgF}_{3}: \mathrm{Mn}$ compounds after irradiation and the energy levels of the complexes in each compound were experimentally determined. The most promising potential dosimeter host material was the near tissue-equivalent $\mathrm{NaMgF}_{3}$. When doped with $\mathrm{Mn}^{2+}$, the compound exhibited RPL via the formation of F-centre/Mn complexes and OSL via several trapping centres. The RPL could be probed independently to the OSL such that the compound could function as a hybrid OSL/RPL dosimeter. In the $\mathrm{NaMgF}_{3}: \mathrm{Ln}$ compounds, RPL occurred via the radiation-induced reduction $\mathrm{Ln}^{3+} \rightarrow \mathrm{Ln}^{2+}$ for $\mathrm{Ln}=\mathrm{Sm}, \mathrm{Dy}$, and $\mathrm{Yb}$. The reduction $\mathrm{Sm}^{3+}$ $\rightarrow \mathrm{Sm}^{2+}$ was highly stable and could be non-destructively probed independently to the OSL. The Sm doped compound also exhibited radiation-induced conductivity that could be coupled with the RL, such that the compound could function as a real-time hybrid optical/electrical dosimeter. Charge kinetics, thermal quenching, and binding energy models were developed and applied to the compounds.

Finally, a two-dimensional readout system was designed and constructed. The capabilities of the system were evaluated using the OSL of $\mathrm{NaMgF}_{3}: \mathrm{Eu}$ and $\mathrm{NaMgF}_{3}: \mathrm{Mn}$. Sensitivities to doses from $<10 \mathrm{mGy}$ to $>1$ Gy were obtained along with sub-millimetre spatial resolutions.
\end{abstract}





\section{Contents}

Contents vii

1 Introduction 1

2 Theoretical Background 5

2.1 Dosimetry ......................... 5

2.1.1 Interactions of Ionising Radiations with Matter . . . . . . . . 5

2.1.2 Dosimetric Quantities . . . . . . . . . . . . . . . . 9

2.1.3 Medical Uses of Ionising Radiation . . . . . . . . . . . . . 10

2.1.4 Modern Dosimeters . . . . . . . . . . . . . . . . . . . . . . . . 12

2.2 Luminescence . . . . . . . . . . . . . . . . . . . . . . . . . 14

2.2.1 Electronic Transitions . . . . . . . . . . . . . . . . . 14

2.2 .2 Band Theory of Solids . . . . . . . . . . . . . . . 16

2.2 .3 Dopants and Defects . . . . . . . . . . . . . . 16

2.2.4 Photoluminescence . . . . . . . . . . . . . . . . 18

2.2.5 Radiophotoluminescence . . . . . . . . . . . . . 21

2.2 .6 Stimulated Luminescence . . . . . . . . . . . . . . . 21

2.2 .7 Persistent Luminescence . . . . . . . . . . . . . . . . . . . . . 23

2.2 .8 Radioluminescence . . . . . . . . . . . . . . . . 23

2.2.9 Radiation-induced Currents . . . . . . . . . . . . . . 25

2.2.10 Transition Metals and Crystal Field Theory . . . . . . . . . . . 26

2.2 .11 Lanthanides . . . . . . . . . . . . . . . . . . . 27

2.3 Fluoroperovskites . . . . . . . . . . . . . . . . . 28

2.4 Summary . . . . . . . . . . . . . . . . . . . 32

3 Experimental Methods $\quad 33$

3.1 Sample Fabrication . . . . . . . . . . . . . . . 33 
3.1 .1 Polycrystalline Samples . . . . . . . . . . . . . . 33

3.2 Materials Characterisation . . . . . . . . . . . . . . 34

$3.2 .1 \quad$ X-ray Diffraction . . . . . . . . . . . . . . . . . . 34

$3.2 .2 \quad$ X-ray Irradiation . . . . . . . . . . . . . . . . 35

3.2 .3 Optical Transmission . . . . . . . . . . . . . . . 35

3.2 .4 Photoluminescence . . . . . . . . . . . . . . 36

3.2 .5 Radioluminescence . . . . . . . . . . . . . . . . . . . 39

3.2.6 Thermoluminescence . . . . . . . . . . . . . . . . 40

3.2 .7 Magnetisation . . . . . . . . . . . . . . 41

3.2 .8 Conductivity ..................... 41

3.3 Dosimeter Systems _ . . . . . . . . . . . . . . . . . . . 42

3.3 .1 CCD Camera Detection . . . . . . . . . . . . . 42

4 Luminescence of $\mathrm{Mn}^{2+}$ in Fluoroperovskite Hosts 43

4.1 Photoluminescence of the Mn-doped Fluoroperovskites . . . . . . . . . . 44

$4.1 .1 \quad \mathrm{NaMgF}_{3}: \mathrm{Mn} \ldots \ldots \ldots \ldots . \ldots \ldots 4$

$4.1 .2 \quad \mathrm{KMgF}_{3}: \mathrm{Mn} \ldots \ldots \ldots \ldots \ldots \ldots \ldots$

$4.1 .3 \quad \mathrm{RbMgF}_{3}: \mathrm{Mn} \ldots \ldots \ldots \ldots \ldots \ldots \ldots \ldots$

$4.1 .4 \mathrm{CsCdF}_{3} \mathrm{Mn} \ldots \ldots \ldots \ldots \ldots \ldots$

4.1.5 Comparison and Summary . . . . . . . . . . . . 55

4.2 F-centre/Mn Complexes in Fluoroperovskites . . . . . . . . . . . . . . 59

$4.2 .1 \quad \mathrm{NaMgF}_{3}: \mathrm{Mn} \ldots \ldots \ldots \ldots$

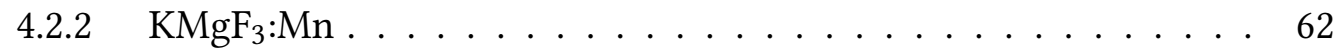

$4.2 .3 \mathrm{RbMgF}_{3}: \mathrm{Mn} \ldots \ldots \ldots \ldots \ldots \ldots$

4.2.4 Comparison and Summary . . . . . . . . . . . . 65

4.3 Dosimetric Properties of $\mathrm{NaMgF}_{3}: \mathrm{Mn} \ldots \ldots \ldots$. . . . . . . . . 69

4.3.1 Optical Absorption . . . . . . . . . . . . . . . . . . . 69

4.3.2 Photoluminescence . . . . . . . . . . . . . . . . 71

4.3.3 Radiophotoluminescence . . . . . . . . . . . . . . . 73

4.3.4 Optically Stimulated Luminescence . . . . . . . . . . . . . . 75

4.3.5 Radioluminescence . . . . . . . . . . . . . . . 78

4.3 .6 Summary and Discussion . . . . . . . . . . . 80

4.4 Dosimetric Properties of $\mathrm{CsCdF}_{3}: \mathrm{Mn} \ldots \ldots \ldots \ldots$

$4.4 .1 \quad$ UV Irradiation . . . . . . . . . . . . . . . . . . 82

$4.4 .2 \quad$ X-ray Irradiation . . . . . . . . . . . . . . . 87 
4.4 .3 Radioluminescence . . . . . . . . . . . . . . . . . 88

4.4 .4 Thermoluminescence . . . . . . . . . . . . . . . 89

4.4 .5 Summary and Discussion . . . . . . . . . . . . . 91

4.5 Chapter Conclusions _. . . . . . . . . . . . . . . . . 93

5 Luminescence of the Lanthanides in $\mathrm{NaMgF}_{3}: \operatorname{Ln} \quad 97$

5.1 Photoluminescence of $\mathrm{NaMgF}_{3}: \mathrm{Ln} \ldots \ldots \ldots$. . . . . . . . . 98

$5.1 .1 \mathrm{NaMgF}_{3}: \mathrm{Sm} \ldots \ldots \ldots \ldots . \ldots \ldots$

$5.1 .2 \quad \mathrm{NaMgF}_{3}: \mathrm{Eu} \ldots \ldots \ldots \ldots 1$

$5.1 .3 \mathrm{NaMgF}_{3}: \mathrm{Dy} \ldots \ldots \ldots \ldots$

$5.1 .4 \quad \mathrm{NaMgF}_{3} \mathrm{Yb} \ldots \ldots \ldots$

5.1.5 Establishing the Lanthanide Levels in $\mathrm{NaMgF}_{3}: \mathrm{Ln}$. . . . . . . . 109

5.1.6 Summary and Discussion . . . . . . . . . . . . . 113

5.2 Dosimetric Properties of $\mathrm{NaMgF}_{3}: \mathrm{Sm} \ldots \ldots \ldots$

5.2.1 Optical Absorption . . . . . . . . . . . . . . . 116

5.2 .2 Magnetisation . . . . . . . . . . . . . . 117

5.2 .3 Optically Stimulated Luminescence . . . . . . . . . . . . . . 120

5.2.4 Radiophotoluminescence . . . . . . . . . . . . . . . . . . 122

5.2 .5 Radioluminescence . . . . . . . . . . . . . . . . . 125

5.2.6 Thermal Quenching of the Luminescence . . . . . . . . . . . . 131

5.2.7 Radiation-induced Conduction . . . . . . . . . . . . 136

5.2 .8 Summary and Discussion $\ldots \ldots \ldots 14 \ldots$

5.3 Chapter Conclusions . . . . . . . . . . . . . . . . . . . . . . 145

6 Development of a Two-dimensional Dosimeter System 149

6.1 Description of the Initial System . . . . . . . . . . . . . . . . . . 149

6.2 2D Dosimetry with $\mathrm{NaMgF}_{3}: \mathrm{Eu} \quad \ldots \ldots \ldots \ldots$

6.2.1 Initial Characterisation . . . . . . . . . . . . . . . . . 151

6.2 .2 2D Characterisation . . . . . . . . . . . . . . 154

6.2 .3 Summary and Conclusions . . . . . . . . . . . . . . 156

6.3 2D Dosimetry with $\mathrm{NaMgF}_{3}: \mathrm{Mn} \ldots \ldots \ldots$

6.3.1 Initial Characterisation . . . . . . . . . . . . . . . . 157

6.3.2 2D Characterisation . . . . . . . . . . . . . . 158

6.3 .3 Summary and Conclusions . . . . . . . . . . . . . . . 161

6.4 Development of the System _ . . . . . . . . . . . . . . . . . 162

6.5 Discussion and Conclusions _. . . . . . . . . . . . . . . 165 
7 Conclusions 169

References 175

A List of Publications

193 


\section{Chapter 1}

\section{Introduction}

Luminescence is widely used as an umbrella term to describe the various phenomena whereby a substance emits light. Luminescence phenomena occur widely in nature, where examples include the bioluminescence of the firefly [1] and the thermoluminescence of diamond [2]. The range of potential mechanisms via which the final result is the emission of light necessitates a large number of sub-classifications, of which photoluminescence, electroluminescence, and optically stimulated luminescence are a few. The existence of these numerous phenomena, coupled with the ubiquity of optics in modern day technologies, has led to the development of many devices that operate using luminescence. Some obvious examples include fluorescent lights [3], light-emitting diodes [4] and lasers [5]. Alternative applications include storage phosphors [6], passive emergency lighting [7], luminescence dating [8], and radiation dose detection [8, 9]. The latter two applications are inextricably linked, as the thermoluminescence used for dating occurs only after exposure to background radiation. This work is largely concerned with the potential applications of luminescence phenomena as regarding radiation dose monitoring.

The initial reports on the observation of X-rays by Röntgen in 1895 [10], the following reports on the phenomenon of radioactivity by Becquerel in 1896 [11], and the discovery of the new highly-radioactive elements radium and polonium by Curie in 1898 [12] motivated the development of an entirely new field of study concerned with the physics and applications of ionising radiations. Ionising radiation is now used regularly in applications related to security [13], diagnostic radiography [14], non-destructive industrial testing [15], and radiation therapy [16], among others.

The use of ionising radiation in a medical context is most relevant to this work. Radiotherapy, the technique by which malignant tissues are destroyed via exposure to 
high doses of highly penetrating X-rays or $\gamma$-rays, relies on very precise beam and dose control in order to minimise damage to surrounding healthy tissues [16]. It is of vital clinical importance to verify the delivered doses in terms of both magnitude and spatial variations as a means of quality assurance. The accidental deliveries of doses to patients exceeding those outlined in treatment plans can have extremely negative consequences, and have demonstrated that experimental quality assurance is essential [17]. Additionally, some novel radiotherapy techniques, such as microbeam radiation therapy, require new devices capable of measuring steep dose gradients over small spatial regions [18]. New devices designed to measure radiation doses, or dosimeters, must be developed in order to keep up with developments in the field of radiation therapy.

The use of luminescence phenomena in dosimetry is a relatively recent scientific development. The thermoluminescence of crystals for the purposes of radiation dose monitoring was suggested in 1953 [19]. Since then, commercial dosimeters operating on the bases of thermoluminescence [20], optically stimulated luminescence [21], and radiophotoluminescence [22] have been developed for a range of radiation-related applications.

The class of ionic compounds known as the fluoroperovskites has proven to provide excellent host materials for several luminescent ions, including transition metals and lanthanides [23-26]. Phenomena including optically stimulated luminescence, thermoluminescence, and radioluminescence have been observed in various samples, all of which could be used for radiation monitoring [27-29]. The particular compound known as neighborite, chemical formula $\mathrm{NaMgF}_{3}$, is considered extremely promising regarding applications in radiotherapy quality assurance due to its near tissue-equivalence, where the compound is known to absorb energy akin to human tissue at high photon energies [30].

This work presents several studies into the luminescence features and mechanisms of some fluoroperovskites doped with various luminescent ions. The effects of exposure to ionising radiation on the luminescence features of the compounds were investigated. One intention of the work was to establish the techniques by which radiation doses could be quantifiably monitored using the luminescence of the compounds. To this end, phenomena including radioluminescence, photoluminescence, and stimulated luminescence were explored in the compounds. A second intention was to elucidate the mechanisms via which radiation dose monitoring was made possible, particularly with regard to the charge kinetics of compounds during and after irradiation. Several analysis techniques and models were developed to better describe the physics of the compounds. A third 
intention was to demonstrate that the materials investigated herein could function as two-dimensional dosimeters suitable for applications in radiotherapy quality assurance. A dosimeter readout system was constructed to achieve this goal.

The main host material of interest throughout this work was $\mathrm{NaMgF}_{3}$ due to its near tissue-equivalence. Other compounds were investigated to provide a point of reference regarding the luminescence and radiation-sensing properties, and in order to improve on the physical descriptions of those phenomena that appeared across multiple compounds. Following this introduction, Chapter 2 will discuss the more pertinent aspects of the background theory necessary in understanding the forthcoming analyses and discussions. The emergence of luminescence as a quantum phenomenon, the physics of ionising radiations, and the properties of the fluoroperovskites will be considered. Chapter 3 will provide details regarding the numerous experimental methods and apparatus used in the characterisation of the materials. Chapter 4 will focus on the luminescence of the fluoroperovskites $\mathrm{NaMgF}_{3}, \mathrm{KMgF}_{3}, \mathrm{RbMgF}_{3}$, and $\mathrm{CsCdF}_{3}$ doped with manganese. The luminescence features before and after irradiation will be discussed in detail and the physical properties of each compound compared. The dosimetric properties of $\mathrm{NaMgF}_{3}: \mathrm{Mn}$ will be presented. Chapter 5 will focus on $\mathrm{NaMgF}_{3}$ doped with various lanthanide ions. The complimentary methods of dose monitoring will be discussed, and information on the band structure of the host compound relative to the set of lanthanides will be presented. The dosimetric properties of $\mathrm{NaMgF}_{3}: \mathrm{Sm}$ will be examined. Finally, Chapter 6 will detail the development of a dosimeter system that was designed and built to detect radiation doses in two-dimensions using the optically stimulated luminescence of doped fluoroperovskites. 



\section{Chapter 2}

\section{Theoretical Background}

The numerous phenomena whereby light is emitted by a substance are a regular topic of study in the scientific world. A majority of these phenomena are physically described via the various forms of luminescence, where some external stimulation of a substance results in the transformation of the stimulating energy into visible light. Luminescence studies have led to the development of several ubiquitous technologies. This work aimed to investigate the luminescence properties of the class of compounds known as the fluoroperovskites and to evaluate potential technological applications. The primary application of interest was ionising radiation detection. The work also aimed to build upon our fundamental understandings of luminescence phenomena in different materials. This chapter introduces the key concepts required to understand the data and discussions presented in later chapters. The possible interactions of ionising radiations with materials, the various luminescence phenomena explored, and the key properties of the fluoroperovskites will be discussed.

\subsection{Dosimetry}

\subsubsection{Interactions of Ionising Radiations with Matter}

By definition, ionising radiation is any radiation possessing sufficient energy to directly, or indirectly, ionise the atoms or molecules of a material [31]. There exist several types of ionising radiation that differ physically. These include electromagnetic waves, such as X-rays, subatomic particles, such as electrons, and ions, such as $\alpha$-particles [31]. In the context of this work we are primarily concerned with electromagnetic radiation. Photons with wavelengths below $100 \mathrm{~nm}(12.4 \mathrm{eV})$ are commonly considered to be of 
sufficiently high energy to be classified as ionising. The high-energy photons interact with materials via several mechanisms that are dependent on both the photon and material properties. The two most significant interaction mechanisms are (1) absorption via the photoelectric effect and (2) inelastic scattering via the Compton effect. These processes are illustrated schematically in figure 2.1 .
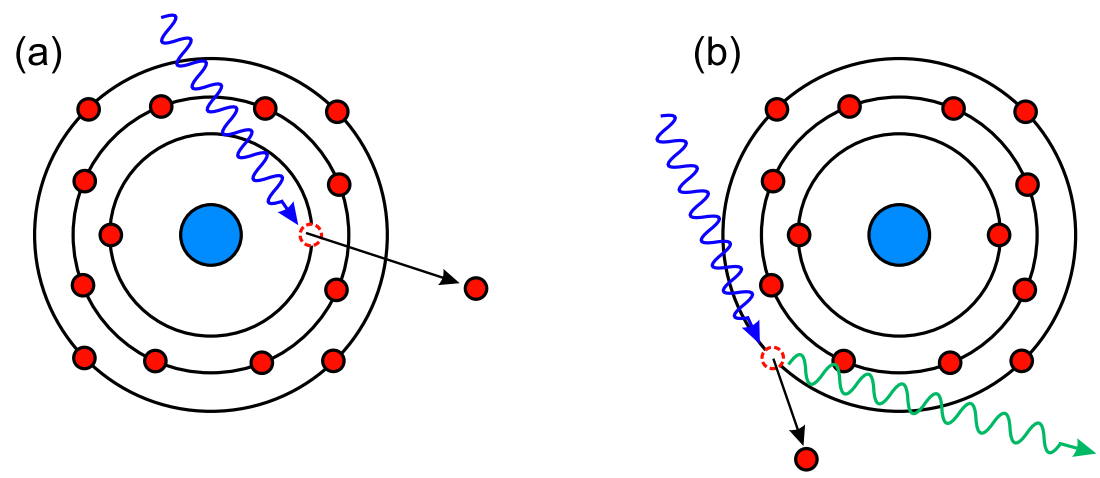

Figure 2.1: Schematic representations of (a) the photoelectric effect and (b) Compton scattering.

When a high energy photon interacts with a tightly bound orbital electron of an atom, the photon may be entirely absorbed by the electron. Consequently, a large amount of energy is transferred to the electron, ultimately ionising the atom. This phenomenon is known as the photoelectric effect, and the ionised electron as a photoelectron [16, 31]. Photoelectrons generated in this way typically possess enough energy to ionise other atoms in the photoelectrons path, such that a single photoelectron is likely to produce a number of secondary ionisation events and secondary photoelectrons.

The probability of a photoelectric absorption interaction occurring is strongly dependent on the energy of the incident radiation and the atomic species impacted by the radiation. This relation can be expressed via the photoelectric attenuation coefficient, commonly written as [31]:

$$
\mu_{\mathrm{PE}} \propto Z^{n} / E^{3}
$$

where $Z$ is the atomic number of the atom, $E$ is the energy of the photon, and $n$ varies between 3 and 4 depending on the incident photon energy. $\mu_{\mathrm{PE}}$ is proportional to the probability of an interaction occurring. Consequently, photoelectric absorption is often the dominant interaction for photon energies $<100 \mathrm{keV}$. As photon energies increases to several hundred $\mathrm{keV}$, other processes become more probable. When the material of 
interest is a compound, we define the effective atomic number, $Z_{\text {eff }}$, as [32]:

$$
Z_{e f f}=\sqrt[n]{\sum_{i} f_{i} Z_{i}^{n}}
$$

where $f_{i}$ is the mass fraction of the element with atomic number $Z_{i}$ and $n \approx 4$. For compounds, $Z_{\text {eff }}$ substitutes for $Z$ in equation 2.1.

When a high-energy photon interacts with a loosely bound electron in a material, inelastic scattering occurs. This process is known as Compton scattering. As this is a scattering phenomenon, only a part of the photon energy is absorbed by the electron. The final product of the interaction must then be (1) a photon scattered an angle $\theta$ relative to the incident photon, with lower energy than the incident photon, and (2) a recoil electron with some kinetic energy supplied by the incident photon. These products are defined via the formula [31]:

$$
\lambda^{\prime}-\lambda=\frac{h}{m_{\mathrm{e}} c}(1-\cos \theta)
$$

where $\lambda$ and $\lambda^{\prime}$ are the wavelengths of the incident and scattered photons, respectively, $h$ is the Planck constant, $m_{\mathrm{e}}$ is the electron rest mass, and $c$ is the speed of light. Again, we may describe the probability of Compton scattering occurring using the relevant attenuation coefficient, $\mu_{\mathrm{CS}}$. However, in contrast to the photoelectric effect, $\mu_{\mathrm{CS}}$ is nearly independent of $Z$ for all atomic species. Furthermore, $\mu_{\mathrm{CS}}$ initially increases with $E$. Consequently, as incident photon energies increase to above several hundred $\mathrm{keV}$, Compton scattering becomes the dominant interaction process.

When a photon has energy equal to or greater than the rest energy of two electrons (i.e. $\geq 1.022 \mathrm{MeV}$ ), an interaction with the strong field of an atomic nucleus may result in the creation of an electron and a positron. This process is known as pair production. The pair production attenuation coefficient, $\mu_{\mathrm{PP}}$, increases with both $Z^{2}$ and $E$ provided the threshold energy condition is met, and therefore pair production dominates at very high photon energies [31].

The linear penetration of photons through matter is ultimately described by an exponential equation [31]:

$$
I=I_{0} \exp \left(-\mu_{\mathrm{T}} x\right)
$$

where $I$ is the transmitted intensity, $I_{0}$ is the incident intensity, $x$ is the penetration depth, and $\mu_{\mathrm{T}}=\mu_{\mathrm{PE}}+\mu_{\mathrm{CS}}+\mu_{\mathrm{PP}}$ is the total linear attenuation coefficient. Per the previous 
discussions, $\mu_{\mathrm{T}}$ will vary significantly depending on the photon energies involved and the material of interest.

When discussing the effects of ionising radiation on matter it is of great importance to account for the fact that, while the aforementioned processes clearly result in the deposition of energy in a material, some energy will subsequently escape the material. This can be made clear by considering the events following a photoelectric absorption interaction. Firstly, a single electron absorbs a photon and is ionised from the atom. The photoelectron continues to ionise additional electrons from additional atoms, depositing some energy with secondary electrons upon each collision. However, as the electrons travel they will radiate energy in the form of Bremsstrahlung radiation, and this radiation and the energy it carries can escape the material. In the case of Compton scattering, the scattered photon may escape the material, such that only the energy absorbed by the electron is retained. The total energy delivered to the material will therefore be lower than the energy of the incident photon in most cases. In order to describe these effects, the mass-energy absorption coefficient, $\frac{\mu_{\mathrm{en}}}{\rho}$, is introduced. The mass-energy absorption coefficient describes the actual energy absorbed by a material, generally as a function of the incident photon energy. Significant variations in $\frac{\mu_{\mathrm{en}}}{\rho}$ occur across different atomic species and compounds, as demonstrated in figure 2.2 for human tissue, the fluoroperovskite $\mathrm{NaMgF}_{3}$, and elemental $\mathrm{Pb}$.

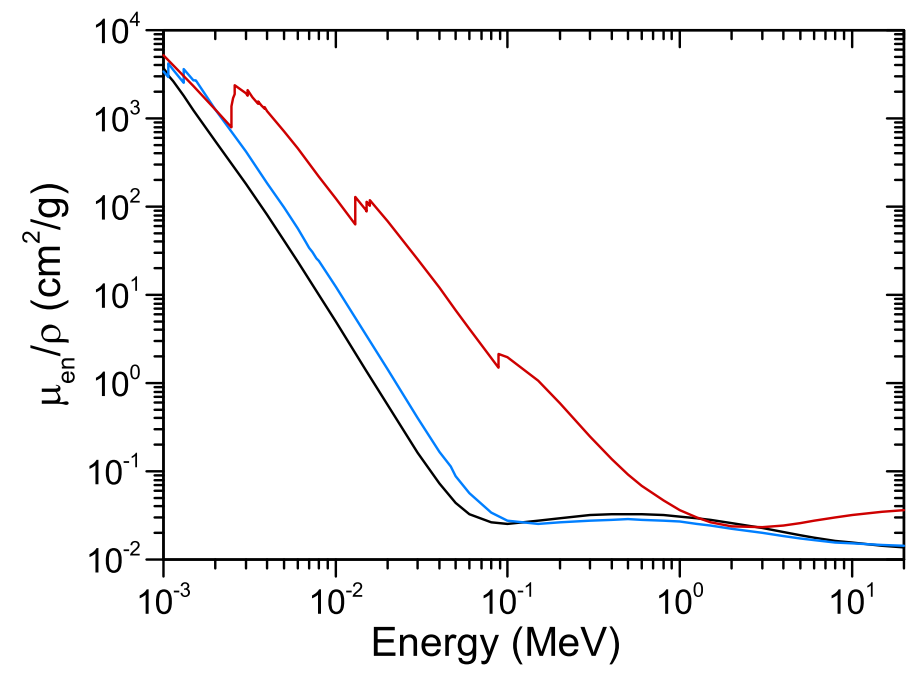

Figure 2.2: Mass-energy absorption coefficient, $\mu_{\mathrm{en}} / \rho$, for tissue (black), $\mathrm{NaMgF}_{3}$ (blue), and $\mathrm{Pb}$ (red). [33]

In the context of medical diagnostics and treatments, it is necessary to understand the effects of ionising radiation on the human body and its constituent components. In 
a broad sense, radiation effects can be separated into two general categories: stochastic effects, and deterministic effects $[16,34,35]$. The severity of each effect varies as a function of the total dose absorbed by the body. The general behaviours of each effect, in terms of severity, are shown in figure 2.3.

Stochastic effects are, by definition, probabilistic. Such effects are most commonly discussed in the context of low radiation doses, e.g. $<0.1 \mathrm{~Gy}$. The most commonly discussed stochastic effect is the induction of cancers [34]. Ionising radiation incident on organic tissues causes the ionisation of molecules within cells and the consequent creation of free radicals [35]. Free radicals are highly reactive and can damage neighbouring cells, resulting in extensive internal damage, such as the creation of chromosomal aberrations [16]. Damaged chromosomes may incorrectly repair and reproduce, ultimately forming cancers. In most cases any radiation-induced cancers will not form until several decades after the radiation exposure occurs [34], highlighting the difficulty in establishing any deterministic method of evaluating low dose exposures. The stochastic effects of radiation are commonly described by the linear no-threshold (LNT) model, whereby the probability of a radiation-induced cancer forming increases linearly with radiation dose at approximately $5.5 \% / \mathrm{Sv}$ [34].

Deterministic effects are those that occur consistently during and after high dose exposures $[16,34,35]$. Such exposures can cause symptoms such as acute radiation syndrome, physical burns, and death. Deterministic effects generally present at doses on the order of 1 Gy and above, whereupon the degree of internal cell damage is sufficient to guarantee mass cell death $[16,36]$. For whole-body doses the mortality rate increases rapidly with dose, diverging significantly from the LNT model (figure 2.3). Despite the apparent dangers, the deterministic effects of radiation are exploited for many applications, including the sterilisation of medical equipment, and more pertinently in the treatment of cancers. The intention of radiotherapy is to induce mass cell death in a malignant tumour, ultimately resulting in the destruction of the cancer, while mitigating damage to the surrounding healthy tissues [16].

\subsubsection{Dosimetric Quantities}

As the sources, interactions, and effects of irradiation can vary significantly, a range of different physical quantities have been used to describe radiation phenomena both historically and presently. In general, these quantities can be broken into several categories: values associated with the source, as in radioactivity; values associated with the field, as in particle fluence; values associated with interactions, as in mass-attenuation coeffi- 


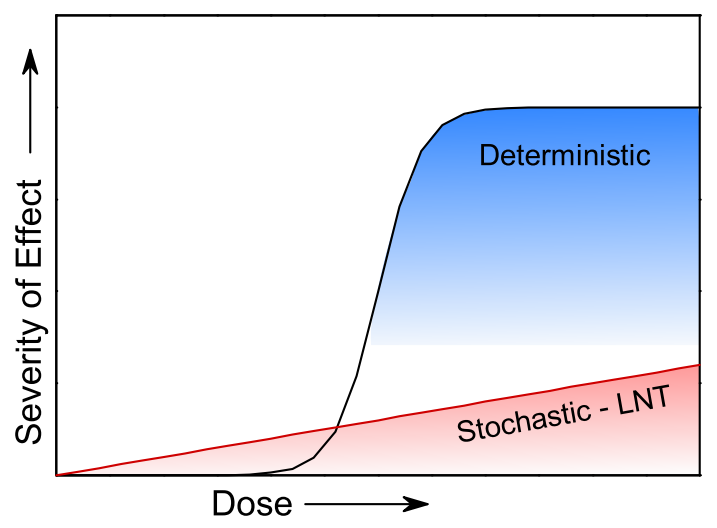

Figure 2.3: Comparison of the linear-no-threshold model for stochastic effects and the threshold model for deterministic effects, versus radiation dose.

cients; values associated with energy transfer, as in absorbed dose; and values associated with radiation protection, as in dose equivalent [31]. In the context of this research we are concerned primarily with those quantities used to describe the absorption of energy by matter.

Throughout this work radiation doses will be discussed in terms of the absorbed dose, $D$, defined as the mean energy imparted to a substance per unit mass [31]. This is mathematically defined as follows:

$$
D=\frac{d \bar{\varepsilon}}{d m}
$$

where $\bar{\varepsilon}$ is the mean energy deposited in a material of mass $m$.

Absorbed dose is typically measured in units of Gray (Gy), where $1 \mathrm{~Gy}=1 \mathrm{~J} / \mathrm{kg}$. The dose rate is given by:

$$
\dot{D}=\frac{d D}{d t}
$$

and is measured in Gy/s. It should be noted that $D$ is by definition specific to the material under discussion. As such, in the discussion of radiation doses, the material of interest should always be specified.

\subsubsection{Medical Uses of Ionising Radiation}

The unique properties of ionising radiation have been exploited medicinally in both diagnostics and treatments. Some applications and techniques are briefly discussed here, in terms of the key science of the technique and the medically beneficial result. Radiation 
doses commonly encountered in medical applications are summarised in table 2.1.

Likely the most prevalent medical application of radiation is that of projectional radiography, i.e. X-ray imaging. A person is exposed to low doses of X-rays in order to capture internal images of the body in a non-intrusive, non-destructive way. Standard radiographs deliver doses on the order of $0.1 \mathrm{mGy}$ to a patient [14] and are considered to have no significant detrimental effect. The diagnostic capabilities of radiography arise from the high penetration probabilities of X-rays. A significant fraction of the X-rays used for this purpose pass through the human body, per equation 2.4, allowing the radiographer to produce a shadow image. Different parts of the body absorb different quantities of the radiation due to their different linear attenuation coefficients. For example, the high calcium content of bone relative to the surrounding tissue provides excellent contrast. In some cases an iodine-based agent is injected into the blood stream to improve the image contrast, in particular when imaging the cardiovascular system. The detectors used for X-ray imaging are specifically designed to be highly sensitive to X-rays in order to decrease the doses required to produce high quality images. As such, diagnostic X-ray detectors are typically composed of materials with high effective atomic numbers.

Computed tomography $(\mathrm{CT})$ is a technique of increasing popularity where $3 \mathrm{D} \mathrm{X}$ ray images are generated, again for diagnostic purposes. This is achieved by obtaining multiple 2D images about an axis, then computing the 3D image. As multiple 2D scans are required the dose delivered to a patient increases significantly, typically up to $1-10$ mGy [14]. Despite the increased risk that comes with higher doses, the use of CT as a diagnostic tool has increased dramatically over the last several decades [14].

Nuclear medicine is the field in which radioactive substances are used for both diagnostics and treatments [16, 37]. Physiological functions may be imaged by detecting the radiations emitted by some substance. Additionally, cancers may be treated by administering radioactive substances directly to the affected area. The doses delivered in these contexts vary greatly depending on the substance and the application [16].

Radiotherapy describes the direct application of ionising radiation to cancers, where the aim is to control or destroy the cancerous tissue [16]. The basic idea is to deliver as high a dose as possible to the target cells in order to destroy the malignant DNA, while simultaneously minimising the dose delivered to the surrounding healthy tissue. This has been achieved in several ways, the most straightforward being the precise control of incident radiation beams. Specific beam geometries and contours may be constructed such that the target volume receives a cumulative dose sufficient to destroy the cancer, while other areas receive significantly lower doses. In some cases cancerous cells have 


\begin{tabular}{ccc}
\hline Treatment & Dose (mGy) & Biological effect \\
\hline \hline Radiography & 0.1 & $\begin{array}{c}\text { Minor risk of cell damage and the eventual } \\
\text { formation of cancers. }\end{array}$ \\
\hline Computed tomography & $1-10$ & $\begin{array}{c}\text { Increased risk of cell damage and the } \\
\text { eventual formation of cancers. }\end{array}$ \\
\hline Radiotherapy & $1000-10000$ & Mass cell death. \\
\hline
\end{tabular}

Table 2.1: Radiation doses received during standard medical procedures and the potential effects on cells.

higher radiosensitivities than healthy cells [38], increasing the likelihood of successful treatment. Additionally, healthy tissues generally repair themselves faster than cancer cells [16], such that the radiation doses used for radiotherapy are often fractionated into a series of smaller doses.

The direct application of radiation is often delivered as an X-ray beam generated by a medical linear accelerator (linac) [16]. The produced photon beams typically have energies on the order of several $\mathrm{MeV}$, and the cumulative doses delivered are typically on the order of 1 - 10 Gy [16]. The doses are necessarily highly destructive to living systems and so great care must be taken in both the planning and treatment stages by a trained radiation oncologist. A mandatory precaution is the quality assurance of the linac irradiations, where precise doses and their distributions must be measured with an accuracy near 99\%. Dosimeters exist specifically for this application. However, the development of novel radiotherapy treatment techniques, e.g. microbeam radiation therapy [18], has highlighted the need for new dosimeter systems capable of accurately measuring complex dose distributions in two- and three-dimensions.

\subsubsection{Modern Dosimeters}

The applications of ionising radiations in many occupations have resulted in the development of numerous devices intended to detect radiation doses, i.e. dosimeters, primarily for health and safety purposes. Here, the state of the art of dosimetry is briefly discussed, primarily in terms of the different devices used for dose detection. There are many dosimeters available commercially [20, 21, 39-41], and a far greater number of materials currently being researched and developed for eventual dosimeter applications [42-48]. This reality reflects the range of circumstances in which radiation dose monitoring is required. 
One of the first developed dosimeters was the ionisation chamber [40], and these devices retain their place as a gold-standard in dose monitoring. The detector is composed of a chamber filled with some gas. A voltage is applied across the chamber, producing an internal electric field. In the absence of irradiation, the gaseous medium is largely nonconductive, such that a near-zero current is measured. Incident radiation ionises the gas, producing free charges and an electric current that is proportional to the real-time dose rate. Some major disadvantages of the device include the high voltages required to generate sufficient currents, the strong sensitivities to humidity and thermal effects, and the limits on miniaturisation. Despite these disadvantages, ionisation chambers are still used worldwide in a range of applications, including radiotherapy quality assurance.

Some occupations, e.g. radiation oncology, involve the worker being exposed to a large number of very low doses over time. In this case, dosimeters are required that monitor the cumulative dose over time, ensuring that the worker is not exposed to a total dose above an established limit. This predicates the field of personal dosimetry. Dosimeters applied in this manner must be unobtrusive and capable of measuring very small doses over significant time periods. Tissue-equivalence is an advantage. This role was once filled by film-badge dosimeters, composed of a piece of film sensitive to radiation, encapsulated in a badge that was then worn on the person throughout their work [49]. The primary advantage of these dosimeters was that doses were monitored passively, where the readout was performed by developing the film after some established monitoring period. However, these films were one-time use and had complicated stability issues.

Electronic dosimeters have largely replaced film. Electronic dosimetry describes the range of devices that interact with radiation to produce a measurable electrical effect. Examples include the diode-based dosimeters and MOSFET dosimeters that use physical changes in the properties of semiconductors to determine dose exposures [41]. These devices typically yield readings in real-time, providing a means of immediately identifying any unintended or emergency exposures, such as those caused by radiation leaks. An up-and-coming dosimeter device suitable for radiotherapy quality assurance is the diamond dosimeter [50-52]. The operating principle of the devices mimics that of the ionisation chamber: a voltage is applied over a thin piece of synthetic diamond, and the electrical current is monitored before and during periods of irradiation.

A significant portion of modern dosimeter research has focused on materials showing stimulated luminescence (SL) phenomena. Stimulated luminescence dosimeters include thermoluminescent dosimeters (TLDs) [20,53] and optically stimulated lumines- 
cence dosimeters (OSLDs) $[21,39,54]$. SL devices operate passively. The sensitive materials store radiation-induced charges within their volume that can later be stimulated with heat (TLDs) or light (OSLDs). The stimulation results in the release of the stored charges, recombination events, and the emission of light, where the emission intensity is proportional to the dose received by the material. There are numerous advantages to such devices. Detection is entirely passive, as no electrical input is required to store the dose information. A wide range of materials show SL phenomena, meaning devices can be tailored to very specific applications. Additionally, devices can be miniaturised to function as precise point dosimeters, or incorporated into films to function as accurate $2 \mathrm{D}$ dosimeters. Commercial examples of OSLDs and TLDs include $\mathrm{Al}_{2} \mathrm{O}_{3}: \mathrm{C}$ [21], $\mathrm{BeO}$ [39], and $\operatorname{LiF}[20]$.

Finally, radiophotoluminescence (RPL) dosimeters have found applications in several fields $[18,22]$. The devices are generally based on measuring the changes in luminescence in silver-doped glasses, where radiation exposure induces oxidation and reduction events in the various silver valences $\left(\mathrm{Ag}^{0}, \mathrm{Ag}^{+}, \mathrm{Ag}^{2+}\right)$. The primary advantage of RPL as a dosimetry technique is that it is passive, all-optical and non-destructive, making it ideal for the monitoring of cumulative radiation doses [22].

\section{$2.2 \quad$ Luminescence}

\subsubsection{Electronic Transitions}

Atoms and ions are composed of a dense atomic nucleus consisting of protons and neutrons, characterised by atomic number $Z$, and electrons that orbit the nucleus, characterised by specific atomic orbital functions. One of the crowning achievements of the $20^{\text {th }}$ century was the advent of quantum mechanics, during which wave-particle duality and the quantisation of orbital energies were established [55]. A consequence of energy quantisation is the emergence of energy gaps between electronic states that are entirely inaccessible to an electron. Transitions between these states occur at fixed energies, and it is this property of atoms that leads to the variety of luminescence features in different materials.

In the mathematical formalism of quantum mechanics, a system can be described in terms of a Hamiltonian operator, $\mathscr{H}$, that operates on electron eigenstates, $\psi_{a}$, with eigenvalues, $E_{a}[55,56]$. The Hamiltonian operator describes the kinetic and potential energies associated with the system. A transition between states is characterised by an 
initial eigenstate $\psi_{i}$ and a final eigenstate $\psi_{f}$, separated by some energy gap $\Delta E$, and the probability of a transition occurring is proportional to $\left|\left\langle\psi_{f}\left|\mathscr{H}_{\text {if }}\right| \psi_{i}\right\rangle\right|^{2}$. A system in an energetically favourable ground state is unlikely to spontaneously transition to an excited state. In contrast, a system in an excited state is likely to spontaneously transition to the ground state, where the energy difference may be ejected from the system as a photon.

A system may be externally excited into a higher energy state via optical stimulation, where the electromagnetic field of a photon may interact with the electric and magnetic dipole moments of an electron to deliver energy to the system. When the photon energy, $E_{\gamma}$, is equal to the energy difference between $\psi_{i}$ and $\psi_{f}$, the transition may occur. However, the likelihood of any transition is dependent on a variety of factors. For example, electric dipole transitions are approximately $10^{5}$ times more likely to occur than magnetic dipole transitions [55]. The conservation laws regarding parity, momentum, energy and spin must also be satisfied, and this condition leads to several selection rules that govern electronic transitions.

An electric dipole driven transition has a probability proportional to the value of $\left|\left\langle\psi_{f}\left|\mathscr{H}_{\text {if }}^{\mathrm{e}}\right| \psi_{i}\right\rangle\right|^{2}$, where $\mathscr{H}_{\text {if }}^{\mathrm{e}}$ is the Hamiltonian for an electric dipole interaction. $\mathscr{H}_{\text {if }}^{\mathrm{e}}$ is of odd parity, such that if the initial and final states are of the same parity, the expression $\left\langle\psi_{f}\left|\mathscr{H}_{\text {if }}^{\text {e }}\right| \psi_{i}\right\rangle$ will be of odd parity and the transition probability will be zero. Therefore, an electric dipole transition requires that the initial and final states change in parity. The opposite occurs for magnetic dipole transitions, where the magnetic dipole Hamiltonian $\mathscr{H}_{\text {if }}$ is of even parity. Magnetic dipole transitions therefore require that the initial and final states must be of similar parity [55].

The electronic states of a system are typically governed by three main interactions: (i) the Coulomb interaction $\left(\mathscr{H}^{\prime}\right)$, (ii) the spin-orbit interaction $\left(\mathscr{H}_{\mathrm{SO}}\right)$, and the interaction with the atomic nucleus $\left(\mathscr{H}_{0}\right)$. These interactions lead to well-defined quantum numbers that summarise the electronic configuration of the system, namely spin $(S)$, orbital angular momentum $(L)$, and total angular momentum $(J)$ quantum numbers. The energy levels can then be described by spectroscopic term symbols of general form ${ }^{2 S+1} L_{J}$, provided the spin-orbit interaction is weak relative to the other interactions. As the spin wavefunction is directional and of odd parity, any transition for which the spin number changes is forbidden. Therefore, allowed transitions must satisfy $\Delta S=0$. Furthermore, in order to conserve orbital momentum we require that $\Delta L=0, \pm 1$, and that $\Delta J=0, \pm 1$, though where $J=0 \leftrightarrow 0$ is forbidden. These additional selection rules are summarised below [55]: 
1. $\Delta S=0$,

2. $\Delta L=0, \pm 1$,

3. $\Delta J=0, \pm 1$, provided $J \neq 0 \longleftrightarrow 0$.

The luminescent species discussed in this work will have their electronic states denoted by the spectroscopic term symbols where possible.

\subsubsection{Band Theory of Solids}

When a large number of atoms coalesce to form a lattice, the periodicity of the atoms and orbital interactions produce energy levels resembling continuous bands [55]. This occurs due to the overlap of atomic orbitals as they are brought closer together. The Pauli Exclusion Principle, which states that no two electrons can occupy exactly the same quantum state, causes the overlapping orbitals to split in energy and, as the number of constituent atoms becomes very large (on the order of $\mathrm{N}_{\mathrm{A}}$ ), these split energies can be approximated by continuous bands. This phenomenon is conceptually illustrated in figure 2.4 (a).

Depending on the chemical and electronic properties of the lattice, the bands may be separated by some energy gap such that there forms two distinct bands, designated the valence band and the conduction band. The energy difference between the highest energy orbital of the valence band and the lowest energy orbital of the conduction band is known as the band gap energy, commonly denoted $\varepsilon_{g}$. Depending on the value of $\varepsilon_{g}$, materials are classified as one of three material types (Fig. $2.4(\mathrm{~b})$ ): 1) insulators, 2 ) semiconductors, and 3) conductors. In conductors, $\varepsilon_{g} \approx 0$ such that electrons can freely travel through the higher energy bands. In insulators, $\varepsilon_{g}$ is on the order of several $\mathrm{eV}$, such that electrons cannot occupy the conduction band in the absence of external stimulation. Semiconductors occupy the intermediate values of $\varepsilon_{g}$, with low band gap energies, and they may or may not conduct depending on the external environment and internal doping. In the context of luminescence studies, insulators and semiconductors are the main materials of interest. In this work we are concerned primarily with highly insulating compounds, where $\varepsilon_{g}>5 \mathrm{eV}$.

\subsubsection{Dopants and Defects}

In the context of this work, dopants are luminescent ions incorporated into a host lattice in relatively low concentrations $(<5 \%)$. The dopant ions have their own unique 
(a)

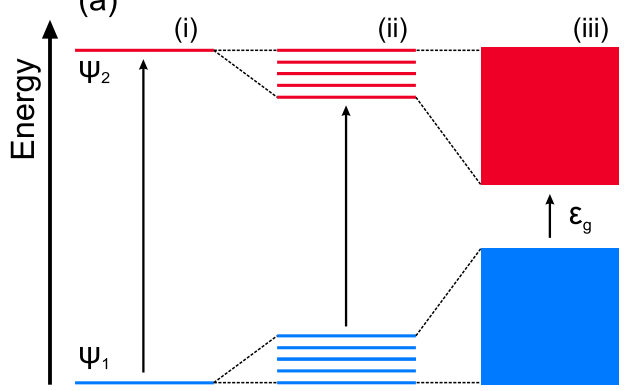

(b)

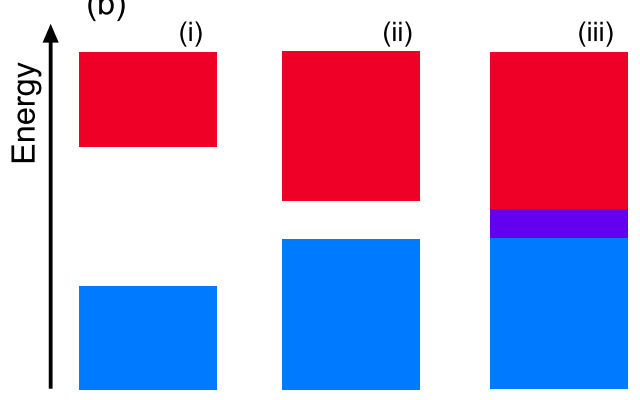

Figure 2.4: (a) The emergence of a band gap as a result of atomic clustering. Blue lines are ground state energy levels and red lines are excited state energy levels for (i) an individual atom, (ii) a cluster of several atoms, and (iii) a crystalline solid. (b) Schematic band gaps of (i) insulators, (ii) semiconductors, and (iii) metals.

electronic structures relative to the ions that constitute the host compound, and consequently introduce new energy levels to the system. As they are spatially localised, either individually or in the form of small clusters, the introduced energy levels remain discrete and can emerge within the band gap of the host compound. This property of dopants gives rise to the efficient, tunable luminescence features observable in a wide range of insulating compounds.

For a dopant ion to incorporate into an ionic host material, it must find some stable configuration within the lattice. In most cases dopants will substitute an ion in the compound that possesses a similar ionic radius and/or a similar electronic valence. Dopants may also occupy interstitial positions in the lattice. In any case, the crystalline lattice will be altered slightly to accommodate the foreign ion, and this may manifest in several ways.

When the ionic radius of some dopant ion, $D$, is different to the ionic radius of the host ion for which it substitutes, $A$, the crystal lattice will often expand or contract in response. If $D$ has a different valence to $A$, e.g. the substitution $D^{3+} \leftrightarrow A^{+}$, the introduced charge imbalance will be compensated for. This property of ionic crystals (charge compensation) has several consequences related to material's band structures and defect production. For highly insulating compounds, the most common consequence of charge compensation is the simple addition, or removal, of an ion with opposite, or equal, charge. As such, adding dopants to materials often introduces ion vacancies or interstitial ions that affect the charge kinetics of the compound. In addition to the structural changes and the emergence of new luminescence features, dopants can affect the charge kinetics within a compound by themselves acting as charge trapping sites and recombination pathways. 
Material's defects can be divided into four main categories: point defects, line defects, planar defects, and bulk defects. The production of some defects in a material during synthesis is practically unavoidable, though precise synthesis techniques can allow for some control over the defect concentrations. Perhaps the most common defect in ionic crystals is the negative ion vacancy e.g. the removal of a constituent $\mathrm{Cl}^{-}, \mathrm{O}^{2-}$, or $\mathrm{F}^{-}$[55]. Removal of the negative ion leaves a vacancy with an effective positive charge that is capable of trapping electrons, forming a structure somewhat analogous to the hydrogen atom. Upon electron capture these centres are known as F-type centres, where in the simplest case a single anion vacancy traps a single electron and an F-centre is formed. F-centre trapping states are located within the band gap and are generally stable, giving rise to broad absorption bands and luminescence features. Mathematical treatments of the centres have been explored by various authors [55]. Most relevant to this work are the findings of Smakula, who provides a means of estimating the F-centre concentration, $N_{\mathrm{F}}$, from the optical absorption, via the formula [57]:

$$
f N_{\mathrm{F}}=0.87 \times 10^{17} \mathrm{~cm}^{2} \alpha_{\mathrm{m}} W_{\mathrm{eV}} \frac{n}{\left(n^{2}+2\right)^{2}},
$$

where $f$ is the oscillator strength of optical transition, $\alpha_{\mathrm{m}}$ is the peak absorption coefficient of the centre, $W_{\mathrm{eV}}$ is the full-width at half-maximum (in $\mathrm{eV}$ ) of the absorption, and $n$ is the refractive index of the material at the peak absorption wavelength. A range of other defects exist, including positive ion vacancies, interstitial ions, antisite defects, and impurity substitutions, all of which affect the material and the luminescence properties to some extent. A schematic illustrating several prominent point defects that form in ionic crystals is shown in figure 2.5 .

\subsubsection{Photoluminescence}

Photoluminescence (PL) is the phenomenon whereby a material absorbs light of some wavelength and subsequently emits light of an equal or higher wavelength [55]. The process is readily understood with reference to the electronic orbitals of individual atoms and ions (figure 2.6 (a)). Optical stimulation can cause an electronic transition, most commonly from the ground state, $\psi_{\mathrm{GS}}$, to some short-lived excited state, $\psi_{\mathrm{ES}}$. The excited state will have some probability of spontaneously returning to the ground state and this relaxation may produce a photon. The probability of such a relaxation gives rise to PL lifetimes that are specific to unique transitions. For example, when the probability of radiative decay from $\psi_{\mathrm{ES}}$ to $\psi_{\mathrm{GS}}$ is high, the average lifetime of $\psi_{\mathrm{ES}}$ will be short $(\sim 1 \mathrm{~ns})$. 


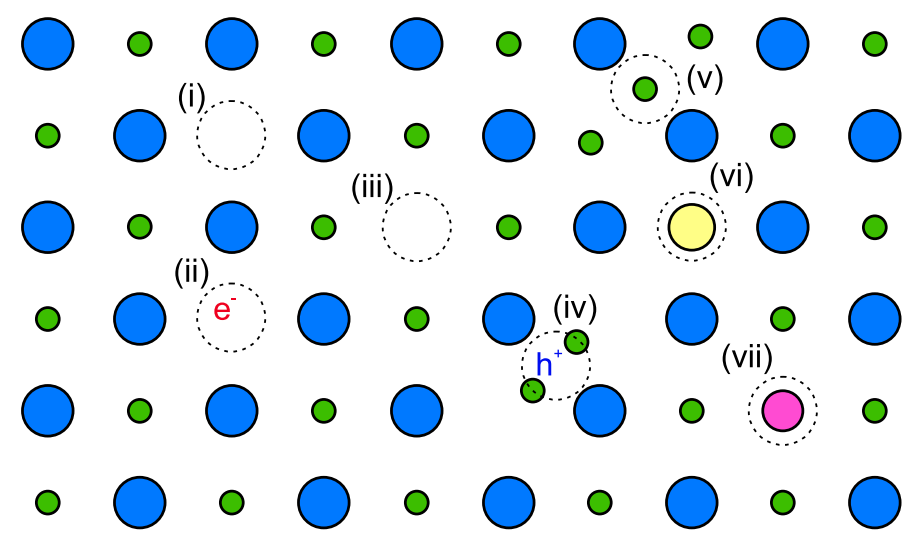

Figure 2.5: Schematic illustrating the structure of several point defects in an ionic crystal. Blue circles are cations and green circles are anions. Dashed circles indicate the defects of interest: (i) negative ion vacancy, (ii) F-centre, (iii) positive ion vacancy, (iv) $\mathrm{V}_{\mathrm{k}}$-centre, (v) negative ion interstitial, (vi) negative ion substitution, (vii) positive ion substitution.

(a)

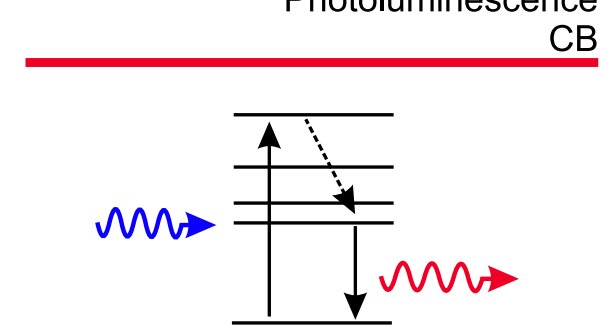

VB

(c)

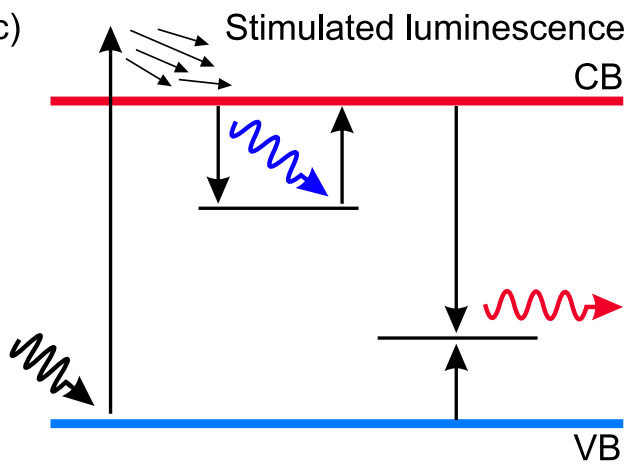

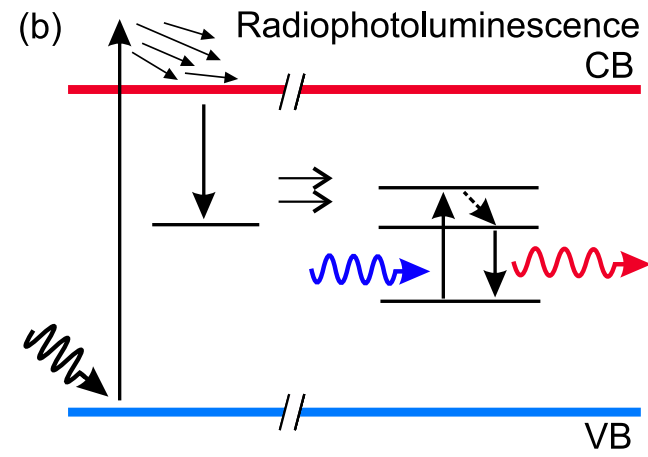

(d)

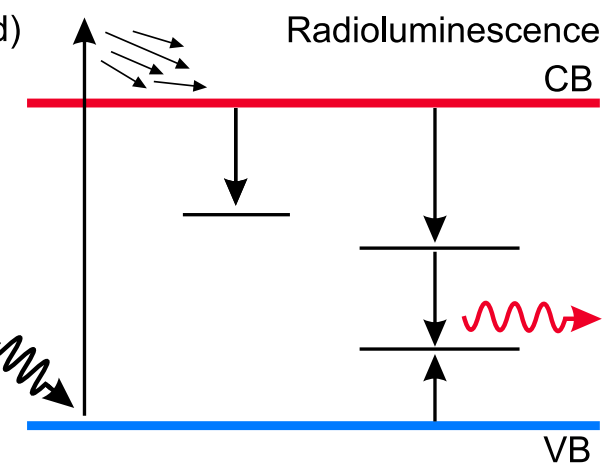

Figure 2.6: Schematic diagrams of the luminescence mechanisms (a) photoluminescence, (b) radiophotoluminescence, (c) stimulated luminescence, and (d) radioluminescence. Blue solid lines represent the valence band and red solid lines represent the conduction band. States within the band gap are represented by black lines. Solid arrows represent possible excitations and decays of electrons. The black, blue, and red waves represent $\mathrm{X}$-ray stimulation, optical stimulation, and optical emission, respectively. 
Consequentially, allowed transitions have characteristically short PL lifetimes, whereas forbidden transitions have characteristically long PL lifetimes ( 1 ms).

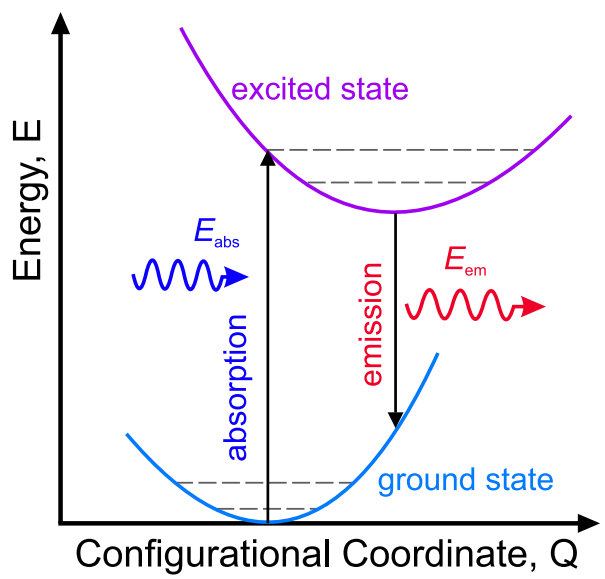

Figure 2.7: Configurational coordinate diagram schematic for a system with a single excited state.

Ions incorporated into a crystal lattice will almost always produce PL emissions at a lower energy than that of the PL excitation due to lattice vibrations known as phonons [55]. Electronic excitations necessarily alter the local electric field of the luminescent ion, leading to a relaxation of the system while an electron is in an excited state. The configurational coordinate model is commonly used to understand this phenomenon, schematically illustrated in the harmonic approximation in figure 2.7. Potential curves for the ground state and excited state are shown as a function of configurational coordinate, $Q$. Both curves have minima at different $Q$ due to the different electron-lattice coupling of each state. In this scheme, optical (radiative) transitions are vertical, and the absorption occurs from the bottom of the ground state to the first available point on the excited state curve. The electron, now in the excited state, non-radiatively relaxes to the minimum of the excited state curve, before radiatively relaxing to the ground state. It is clear that in this case the energy required to excite the system, $E_{\mathrm{abs}}$, is greater than the energy of the emitted photon, $E_{\mathrm{em}}$, due to the intermediate non-radiative relaxation steps.

As the PL characteristics of an ion are directly dependent on its electronic structure, PL spectroscopy has proven effective in fingerprinting luminescent species and the crystalline environments that they occupy. The energy levels of free ions are well established such that comprehensive reference sets are available for comparison to experimental data [55]. Perturbations to the free ion levels are expected due to various influences, including crystal field effects and the presence of defects in most compounds. A great deal 
of physical information can be obtained about a material via PL spectroscopy, hence its universal use in luminescence studies.

\subsubsection{Radiophotoluminescence}

Radiophotoluminescence (RPL) occurs when exposure to ionising radiation produces new PL-active centres in a material $[22,58]$ and is therefore a sub-category of PL. The standard example of RPL is one of valence conversion, e.g. the radiation-induced reduction of a trivalent ion, $D^{3+}$, to its divalent state, $D^{2+}$, where $D^{2+}$ exhibits PL under an appropriate optical excitation [43] (figure 2.6 (b)). RPL therefore provides a very straightforward means of measuring radiation doses, as the emission intensity of the RPL centre will generally increase with total radiation dose up to some saturation dose. The major advantage of RPL as a dosimetry tool over alternative luminescence-based mechanisms is the stability of the effect and the ease of readout. RPL centres can commonly be non-destructively probed using low-energy stimulation wavelengths [43], meaning a material can be read multiple times and intermittently evaluated even while cumulative doses are monitored. When dose monitoring is completed, the RPL centres can be reverted to their initial, pre-irradiation configuration via high-energy optical stimulation or thermal stimulation, and therefore RPL dosimeter compounds tend to function as reusable dosimeters. Common RPL dosimeter materials are mostly limited to the silverdoped glasses [22, 58], where dose monitoring occurs via PL analysis of the various Ag valences that form during irradiation.

\subsubsection{Stimulated Luminescence}

Stimulated luminescence (SL) phenomena occur when a material traps radiation-induced free charges and can subsequently be stimulated into releasing those charges, where the recombination process results in the emission of light $[8,9]$ (figure 2.6 (c)). As SL cannot occur without the initial production of free charges, the phenomenon is uniquely suited to radiation monitoring and imaging, and has found commercial applications in the field $[21,39,54]$. The general process is described by figure 2.6 (c). Ionising radiation generates free charges in the conduction and valence bands of a host compound that will travel some finite distance before they are trapped by a defect or recombine with a charge carrier of opposite sign. SL can occur only in the former case, when charges are trapped in states within the band gap, and the potential barrier is sufficient to prevent the emptying of the traps in the absence of some external stimulation. The energy required 
to liberate the charge from the trap, i.e. the trap depth, determines the stability of the trap at room temperature, where trap lifetimes can range from fractions of seconds to centuries. However, the energy required to liberate the trapped charges can be provided in the form of optical or thermal stimulation. The choice of either optical or thermal stimulation gives rise to the phenomena of optically stimulated luminescence (OSL) [8] and thermoluminescence (TL) [9], respectively, both of which are frequently exploited in radiation dose monitoring. As charges are collected during irradiation without readout, SL provides a means of passive dose monitoring.

SL requires that recombination centres exist, such that when trapped electrons and holes are released and recombine the energy escapes in the form of light. Recombination centres can be intrinsic defects, e.g. the $\mathrm{F}^{+}$-centre in $\mathrm{Al}_{2} \mathrm{O}_{3}$ [59], or dopants, e.g. $\mathrm{Eu}^{2+}$ in $\mathrm{KBr}: \mathrm{Eu}$ [8]. In the latter case the SL emissions and the PL emissions will have similar features, where any minor variations will be due to the influence of defects localised to the dopant that are necessary for SL to occur. SL also requires the presence of electron and hole traps. The means of monitoring radiation doses is largely dependent on the concentration of defects. As the material is exposed to radiation, the concentration of trapped charges increases in a proportional manner. However, for sufficiently high radiation doses, the concentration of trapped charges will approach the total trap concentration and the SL intensity will saturate. Therefore, the radiation dose response behaves asymptotically, placing an upper limit on the dose any particular compound can accurately measure. Furthermore, SL processes are inherently destructive of the stored signal, as the concentration of trapped charges is depleted upon measurement. Consequently, SL dosimeters must be manufactured to be resistant to any environmental bleaching of the stored charges.

The most common SL technique used for dosimetry is continuous wave OSL (CWOSL) [8]. A material is continuously stimulated with light of a constant intensity, while the OSL emission intensity is monitored as a function of time. The emission follows a characteristic decay curve as the concentration of trapped charges decreases. The structure of the decay is dependent on several factors. In the simplest case, where a single trap is stimulated and responsible for the OSL emission (figure 2.6 (c)), the OSL vs. time can be described by a standard exponential decay.

After a material is irradiated there will be some concentration of filled traps, $n_{t}$, that can be optically excited into recombining via some luminescent centre, with a probability $p$, during optical stimulation at a constant intensity. In this case the concentration of 
traps will deplete according to:

$$
\frac{d n_{\mathrm{t}}}{d t}=-p n_{\mathrm{t}}
$$

Equation 2.8 has exponential solutions. The OSL intensity is directly dependent on the rate at which the trapped charge recombines, such that the OSL intensity, $I_{\mathrm{OSL}}$, will vary as:

$$
I_{\mathrm{OSL}}(t) \propto\left|\frac{d n_{\mathrm{t}}}{d t}\right|=p n_{0} \exp (-p t) .
$$

However, most materials contain several different trapping defects, multiple recombination pathways, and exhibit significant rates of retrapping. The OSL decay curves are complicated by these features.

\subsubsection{Persistent Luminescence}

Persistent luminescence (PersL) can be described as a subset of SL. When free charges are trapped by defects with very small trap depths, ambient temperatures may be sufficient to liberate the charges and produce recombination luminescence [7]. In this case, trapped charges begin to escape and recombine during and immediately after the irradiation period. PersL is commonly referred to as afterglow, and is the mechanism that gives rise to many glow-in-the-dark technologies, though where irradiation comes in the form of UV or optical stimulation rather than X-ray stimulation. The appearance of PersL from a material immediately indicates that room temperature traps exist, complicating any other radiation-induced luminescence processes. Practical applications of PersL in the realm of radiation detection have not been thoroughly explored, though the intensity of the PersL decays should be proportional to the total radiation dose in the same way as the other SL phenomena.

\subsubsection{Radioluminescence}

Radioluminescence (RL) is the phenomenon whereby light is promptly emitted during radiation exposure. In the general case, the emission intensity will be proportional to the real-time dose rate, and therefore $\mathrm{RL}$ is useful in the active monitoring of radiation doses $[60,61]$. RL occurs when radiation-induced free charges (photoelectrons + secondary electrons) directly recombine via some luminescent centre. A schematic of a one-trap, one-recombination centre system exhibiting RL is shown in figure 2.6 (d). 
Radiation incident on a RL-active material will generate a large number of excited electrons in the conduction band of the host compound. Similar to the discussed SL processes, the charges will migrate via the host bands until they reach a recombination centre or are trapped by some defect. The recombination of electrons and holes via a luminescent centre results in energy transfer to the centre, leaving it in an excited state from which radiative decay is possible. As the recombination centres observed in RL are often PL active, the luminescent species responsible for RL can often be identified via PL spectroscopy.

In the ideal case, all radiation-induced electrons and holes would recombine via a single luminescent ion, such that the emission intensity was directly proportional to the radiation dose rate. However, the presence of defects in materials often complicates the time-dependence of RL emissions. The concentrations of electrons $\left(n_{\mathrm{c}}\right)$ and holes $\left(m_{\mathrm{v}}\right)$ in the conduction and valence bands, respectively, will be affected by the accumulation of charge in traps. The time-dependencies of $n_{\mathrm{c}}$ and $m_{\mathrm{v}}$, along with the concentration of trapped charges, $n_{\mathrm{t}}$, are described by the following equations:

$$
\begin{gathered}
d n_{\mathrm{c}} / d t=\Gamma \dot{D}-R_{\mathrm{R}} N_{\mathrm{R}} n_{\mathrm{c}} m_{\mathrm{v}}-d n_{\mathrm{t}} / d t \\
d m_{\mathrm{v}} / d t=\Gamma \dot{D}-R_{\mathrm{R}} N_{\mathrm{R}} n_{\mathrm{c}} m_{\mathrm{v}} \\
d n_{\mathrm{t}} / d t=A_{\mathrm{t}} n_{\mathrm{c}}\left(N_{\mathrm{t}}-n_{\mathrm{t}}\right)
\end{gathered}
$$

where $\Gamma$ is the number of electron-hole pairs generated per unit dose, inversely proportional to the band gap energy, $\dot{D}$ is the radiation dose rate, $N_{\mathrm{R}}$ and $N_{\mathrm{t}}$ are the total concentration of recombination centres and traps, respectively, $R_{\mathrm{R}}$ is related to the rate of electron-hole recombination at the recombination centre, and $A_{\mathrm{t}}$ is related to the rate at which the trap captures conduction band electrons.

The RL emission is then

$$
I_{\mathrm{RL}} \propto R_{\mathrm{R}} N_{\mathrm{R}} n_{\mathrm{c}} m_{\mathrm{v}}
$$

with some constant of proportionality related to the luminescence centre itself (e.g. the transition probability) and the configuration of the readout optics.

Real systems often prove more complicated than the one trap, one recombination centre model suggests. The presence of several defects will produce traps with differ- 
ent concentrations and trapping probabilities. Additionally, RL emissions may occur via several recombination pathways. Determining the dose rate directly is complicated by the time-dependence of the emissions. To minimise this effect, some compounds are delivered a high priming dose in order to saturate the trap concentrations [27]. In other cases, algebraic manipulations of distinct emission intensities may be employed in order to achieve a signal that does not vary in time [62]. RL has been exploited in the development of several fibre-optic dosimeters [15, 46, 63], though these often suffer from the aforementioned effects, as well as undesirable luminescence from Cherenkov radiation and the fibre itself.

\subsubsection{Radiation-induced Currents}

Radiation-induced currents (RICs) are analogous to the photocurrents produced in many semiconducting compounds upon exposure to light. As incident radiation on wide-band gap materials excites electrons to the conduction band by either the photoelectric effect or Compton scattering, the application of an electric potential across the material should result in a flow of charges and an electric current [64]. The intensity of the generated current will be largely dependent on the configuration of the setup, e.g. the thickness of the sample, quality of the electrical contacts, voltage etc., and hence the effect is often discussed in terms of radiation-induced conductivity, where conductivity is a materialspecific quantity. The intensity of the induced conductivity is proportional to the band gap of the material, as the number of electron-hole pairs produced per ionisation event is directly related to the band gap. The energy required to create a single electron-hole pair is typically estimated as $3 \times \varepsilon_{g}$, such that a single X-ray photon with an energy of $\sim 20 \mathrm{keV}$ will produce several thousand electrons.

The dose dependence of RICs is generally described by the Fowler equation $[51,52]$ that may be written as

$$
i_{\mathrm{RIC}}=\gamma \dot{D}^{\Delta}
$$

where $i_{\text {RIC }}$ is the radiation-induced current, $\gamma$ is a constant, $\dot{D}$ is the radiation dose rate, and $\Delta$ is a constant between 0.5 and 1 . The value of $\Delta$ is indicative of the trap distribution throughout the material, where $\Delta=0.5$ in the absence of traps or several recombination centres, and $\Delta \longrightarrow 1$ when traps and other centres have a significant influence over the carrier lifetimes and concentrations. 


\subsubsection{Transition Metals and Crystal Field Theory}

Sections 2.2.1 and 2.2.2 introduced the concept of quantised electronic orbitals, where an electron can only transition between well-defined states with fixed energies relative to the ground state. The orbital energies of the valence electrons of a free ion are defined primarily by their interactions with the atomic nucleus and with other electrons, such that the Hamiltonian for the free ion my be written

$$
\mathscr{H}_{\mathrm{F}}=\mathscr{H}_{0}+\mathscr{H}^{\prime}+\mathscr{H}_{\mathrm{SO}}
$$

where $\mathscr{H}_{0}$ is the interaction with the nucleus, $\mathscr{H}^{\prime}$ is the Coulomb interaction, and $\mathscr{H}_{\text {SO }}$ is the spin-orbit interaction.

Incorporating an ion into a lattice introduces additional interactions that will perturb the energy levels relative to the free ion. The perturbations are commonly described using the concept of a crystal field [55]. The crystal field imposed on a luminescent ion is mostly composed of the static electric field produced by the adjacent ligands. It is assumed that the ligands are fixed at a mean distance from the ion of interest. The Hamiltonian of the system is then

$$
\mathscr{H}=\mathscr{H}_{\mathrm{F}}+\mathscr{H}_{\mathrm{CF}}
$$

where $\mathscr{H}_{\mathrm{CF}}$ is the crystal field Hamiltonian that depends on the number, orientation, and fields produced by the neighbouring ligands. Depending on the magnitude of $\mathscr{H}_{\mathrm{CF}}$ relative to $\mathscr{H}_{\mathrm{F}}$, we specify the crystal field to be strong, intermediate, or weak.

Crystal field theory is particularly useful in the study of transition metal ions characterised by valence electrons occupying partially filled $3 d$ shells, including as $\mathrm{Mn}^{2+}$, $\mathrm{Fe}^{3+}$, and $\mathrm{Ni}^{2+}$. The $3 d$ levels are typically low-lying relative to the ground state of the ion such that intraconfigurational $3 d^{n} \longrightarrow 3 d^{n}$ transitions occur at energies corresponding to the visible region of the electromagnetic spectrum. The $3 d$ electrons are exposed to, and consequently strongly interact with, the crystal field. This leads to a significant breaking of the degeneracy of the five $d$-orbitals due to their different spatial configurations, and transition metals in solids are typically analysed by considering the case of a strong crystal field.

In an octahedral $\left(O_{h}\right)$ crystal field, characterised by $M \mathrm{~F}_{6}$ octahedra, where $M$ is a transition metal, the $d$-orbitals are split into a pair, denoted $e_{g}$, and a triplet, denoted $t_{2 g}$, as illustrated in figure 2.8 (a) [55]. The energy separation between the two components defines the crystal field splitting parameter $10 D q$. 

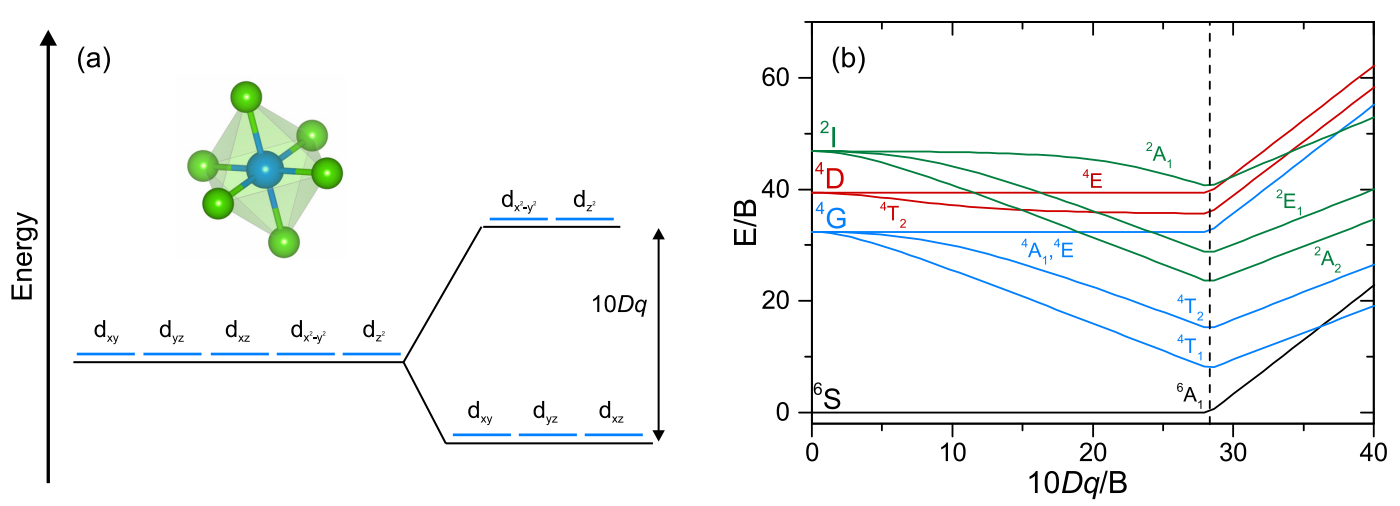

Figure 2.8: Crystal field splitting for $3 d^{n}$ ions. (a) Energy of the $3 d$ orbitals in a spherical crystal field (degenerate) and in an octahedral crystal field (split by $10 D q$ ). (b) TanabeSugano diagram for $3 d^{5}$ ions, e.g. $\mathrm{Mn}^{2+}$.

Analyses of the luminescence spectra allow one to determine the degree of the crystal field splitting and therefore obtain information on the local environment about the ion. The work of Tanabe and Sugano [65] assists greatly in these analyses, where the Tanabe-Sugano diagrams for all $3 d^{n}$ ions may be referenced. These diagrams describe the splitting of the energies of the free ion states as the crystal field strength increases, as shown in figure 2.8 (b) for the $3 d^{5}$ configuration, in terms of both $10 D q$ and the Racah parameter $B$, where $B$ describes the magnitude of the inter-electron repulsion for the transition metal in the $M_{6}$ structure.

\subsubsection{Lanthanides}

The lanthanides $(\mathrm{Ln})$ constitute a series of chemical elements, ranging from La to Lu (atomic numbers 57-71), that exhibit unique physical and luminescence properties that have made them useful in several applications, including storage phosphors [66] and dosimeters [28]. The outer electronic configuration of the lanthanides is $5 s^{2} 5 p^{6} 4 f^{n} 5 d^{1} 6 s^{2}$ and many of their properties arise from the partially filled $4 f$ shell that populates for increasing atomic number [55]. Lanthanide ions typically lose their $5 d$ and $6 s$ electrons, giving rise to a range of possible intraconfigurational $4 f^{n} \longrightarrow 4 f^{n}$ transitions that can produce luminescence. In contrast to the $3 d$ shells of transition metals, the opticallyactive $4 f$ levels are well shielded from the crystal field by the outer $5 s$ and $5 p$ electrons. Perturbations to the free ion energy levels are therefore described by weak crystal field interactions, such that the energy levels vary only slightly across different host compounds. Other possible transitions include $4 f^{n} \longrightarrow 4 f^{n-1} 5 d^{1}$, where the position of the unshielded $5 d$ level varies significantly across different ions and host compounds. 
Lanthanides most commonly incorporate into host materials in their highly stable trivalent states, $\mathrm{Ln}^{3+}$, and a wealth of data are available for the ions in a wide range of different compounds [67-71]. The work of Dieke [71] led to the development of the socalled Dieke diagrams that provide the energy levels of all $\mathrm{Ln}^{3+}$ in a compound. However, as crystal field effects are minimal between hosts, the diagrams can be used to approximate the energy levels of any $\mathrm{Ln}^{3+}$ in any host compound, and therefore have been incredibly useful in the study of lanthanide luminescence for over 50 years. The Dieke diagram for all $\mathrm{Ln}^{3+}$ in $\mathrm{LaCl}_{3}$ is shown in figure 2.9.

The trivalent lanthanides may exhibit alternative stable oxidation states, such as $\mathrm{Ce}^{4+}$ and $\mathrm{Eu}^{2+}$. As the $\mathrm{Ln}^{3+}$ ions are stable in most compounds, it is possible in some cases to alter the valence via some external stimulation such as thermal treatment or exposure to ionising radiation. The $\mathrm{Ln}^{3+}$ ions may act as electron or hole traps in a compound [67], and may lead to SL and RPL phenomena. $\mathrm{Ln}^{2+}$ ions tend to have $5 d$ levels at significantly lower energies than their $\mathrm{Ln}^{3+}$ counterparts and therefore exhibit additional luminescent transitions that are optically accessible [55]. Compared to the $\mathrm{Ln}^{3+}$ ions, few data exist regarding a majority of the $\mathrm{Ln}^{2+}$ ions [72], and thus the study of divalent lanthanides is encouraged where possible.

\subsection{Fluoroperovskites}

The fluoroperovskites are a class of ionic compounds characterised by the chemical formulae $A B F_{3}$ and crystal structures similar to that of the mineral perovskite $\left(\mathrm{CaTiO}_{3}\right) . A$ and $B$ are monovalent and divalent cations, respectively, and $\mathrm{F}$ is fluorine. Due to their ability to retain a range of luminescent ions, it has been proposed that the fluoroperovskites could be useful in a range of technologies and applications, including solid state lasers [73], upconverting crystals [74], and radiation detectors [28]. Interesting physical properties have also presented in some compounds, including the formation of defectdopant complexes that are not yet completely understood [57, 75, 76]. Recent research has emphasised the capability of some compounds to function as efficient OSL and RL dosimeters due to the versatility of the intrinsic defects and adjustable luminescence features [28, 30, 77, 78].

$A B \mathrm{~F}_{3}$ compounds are straightforward to synthesise via the high temperature melting of fluoride precursors and are therefore well suited to mass production in the case of commercialisation. They make excellent host compounds for luminescent ions, due largely to their transparency through the optical and UV spectral regions that arises 


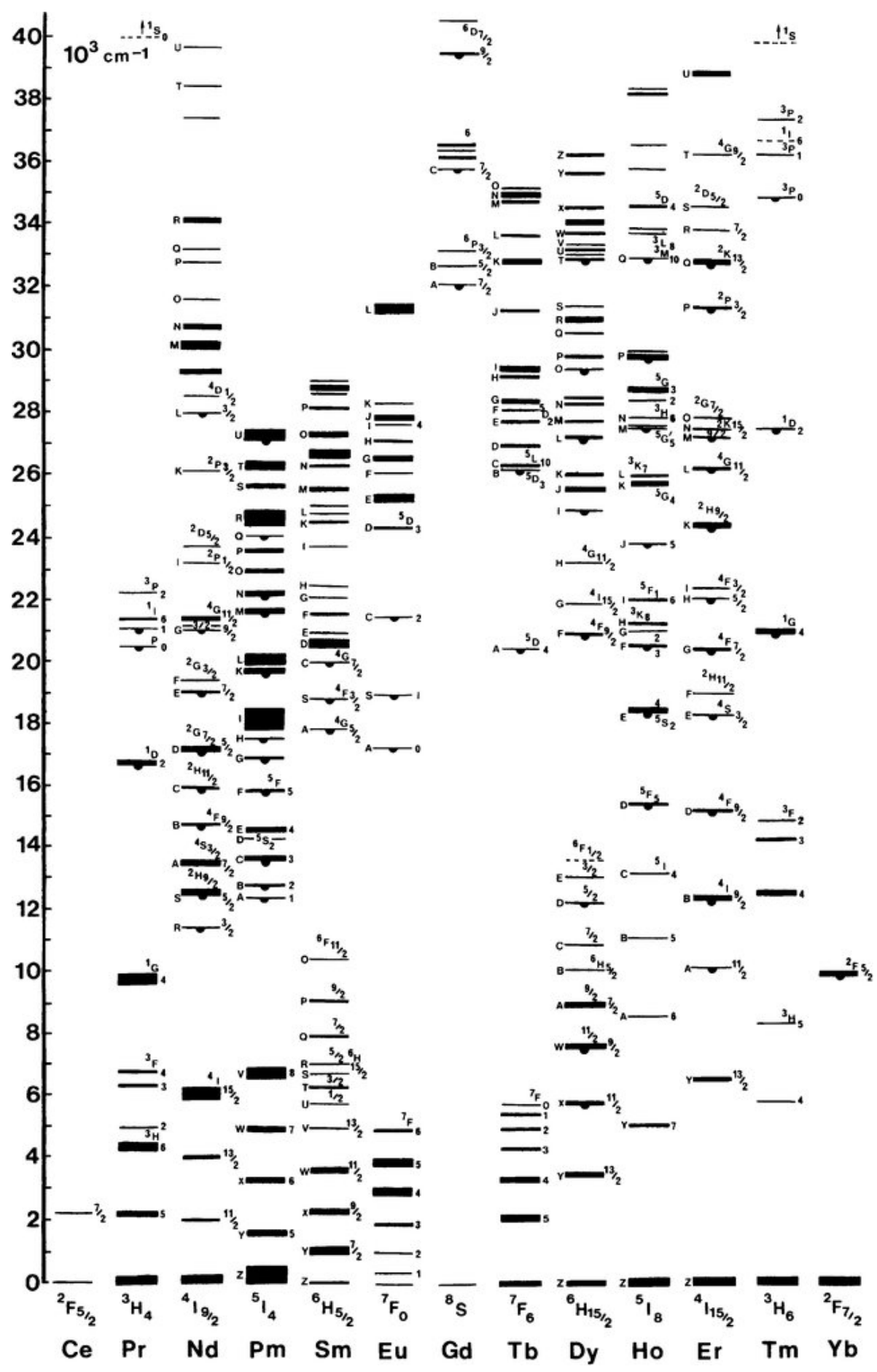

Figure 2.9: Dieke diagram for the trivalent lanthanides in $\mathrm{LaCl}_{3}$, adapted from [55]. 
from their large band gaps and lack of optically-active intrinsic defects.

In the ideal case, $A B \mathrm{~F}_{3}$ compounds would crystallise with a cubic structure, such that the $A$ cation occupies a site of 12 -fold coordination and the $B$ cation occupies a site of 6-fold coordination (i.e. octahedral symmetry). However, the numerous possible $A$ and $B$ cations diversify the range of possible structures and can ultimately modify the crystal field and site symmetries. The tolerance factor, $t_{\mathrm{G}}$, estimates the phase of a perovskite depending on its constituent ions, and can be expressed as [79, 80]:

$$
t_{\mathrm{G}}=\frac{r_{\mathrm{A}}+r_{\mathrm{F}}}{\sqrt{2}\left(r_{\mathrm{B}}+r_{\mathrm{F}}\right)}
$$

where $r_{\mathrm{A}}, r_{\mathrm{B}}$ and $r_{\mathrm{F}}$ are the ionic radii of the $\mathrm{A}, \mathrm{B}$, and $\mathrm{F}$ sites, respectively. If $0.7<t_{G}<$ 0.9 , the compound is most likely to crystallise in an orthorhombic structure. In this case, the relatively small radius of the $A$ cation induces a rotation of the $B \mathrm{~F}_{6}$ octahedra and so exhibits reduced site symmetry. If $0.9<t_{\mathrm{G}}<1$ the compound likely has the ideal cubic structure. For $t_{\mathrm{G}}>1$ the compound will likely adopt a hexagonal structure, producing differently bound $B \mathrm{~F}_{6}$ octahedra and also changing the symmetry of the $A$ site.

Materials investigated in this work include $\mathrm{NaMgF}_{3}, \mathrm{KMgF}_{3}, \mathrm{RbMgF}_{3}$, and $\mathrm{CsCdF}_{3}$. Using the effective atomic radii given by Shannon for 12 -fold coordinated $A$ sites and 6fold coordinated $B$ sites [81], the tolerance factors are calculated to be $0.94,1.03,1.06$, and 1.00, for $\mathrm{NaMgF}_{3}, \mathrm{KMgF}_{3}, \mathrm{RbMgF}_{3}$, and $\mathrm{CsCdF}_{3}$, respectively [80]. However, it has been proposed that the tilting of the $\mathrm{MgF}_{6}$ octahedra in $\mathrm{NaMgF}_{3}$ reduces the coordination of the Na site from 12 to 8 by shifting the position of the $\mathrm{Na}^{+}$ion [82]. In this case $t_{\mathrm{G}}=0.87$, within the range of tolerance factors expected for the orthorhombic structure.

The crystal structures for each compound are illustrated in figure 2.10 and are wellestablished. The band gaps of each compound have been estimated by a range of experiments, and are approximately $\varepsilon_{g}=12 \mathrm{eV}$ [83], $\varepsilon_{g}=11.1 \mathrm{eV}$ [84], $\varepsilon_{g}=10.5 \mathrm{eV}$ [85], and $\varepsilon_{g}=5.5 \mathrm{eV}$ [86], for $\mathrm{NaMgF}_{3}, \mathrm{KMgF}_{3}, \mathrm{RbMgF}_{3}$, and $\mathrm{CsCdF}_{3}$, respectively. $\mathrm{NaMgF}_{3}$ has approximate lattice parameters $a=5.36 \AA, b=5.49 \AA, c=7.67 \AA$ and space group Pbnm [87]. $\mathrm{KMgF}_{3}$ has approximate lattice parameter $a=3.99 \AA$ and space group $\operatorname{Pm} \overline{3} m$ [62]. $\mathrm{RbMgF}_{3}$ has approximate lattice parameters $a=5.83 \AA, b=5.83 \AA, c=14.20 \AA$ and space group $\mathrm{P}_{3} / \mathrm{mmc}$ [88]. $\mathrm{CsCdF}_{3}$ has approximate lattice parameter $a=4.47 \AA$ and space group $P m \overline{3} m$ [89].

Of particular interest for dosimetry applications is $\mathrm{NaMgF}_{3}$ due to its near tissueequivalence, characterised by a low effective atomic number $\left(Z_{\text {eff }}=10.39\right)$ and massenergy absorption close to tissue (figure 2.2). Per section 2.1.1, tissue-equivalent materi- 
als will quantitatively absorb energy via interactions with ionising radiation in a similar manner to human tissue $\left(Z_{\text {eff }} \approx 7.4\right)$, making them ideal for medical dosimetry. The other compounds are studied in order to establish systematic trends across the series of fluoroperovskites regarding their luminescence and radiation-sensing features.

(a)

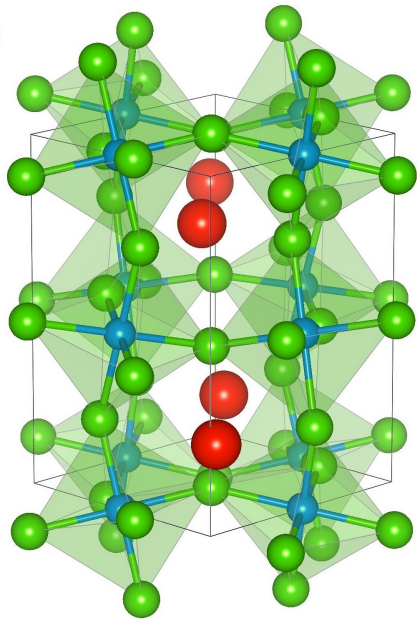

(c)

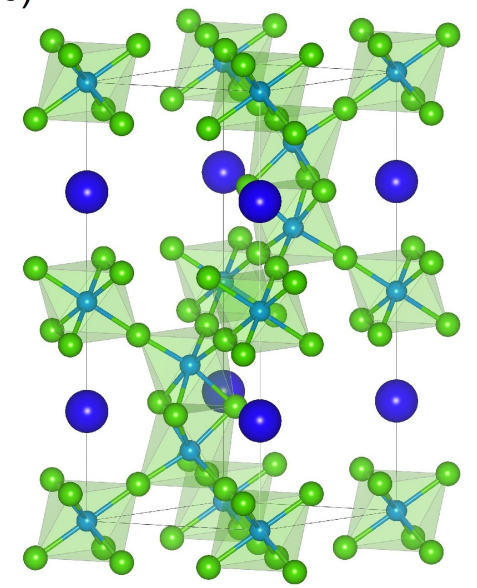

(b)

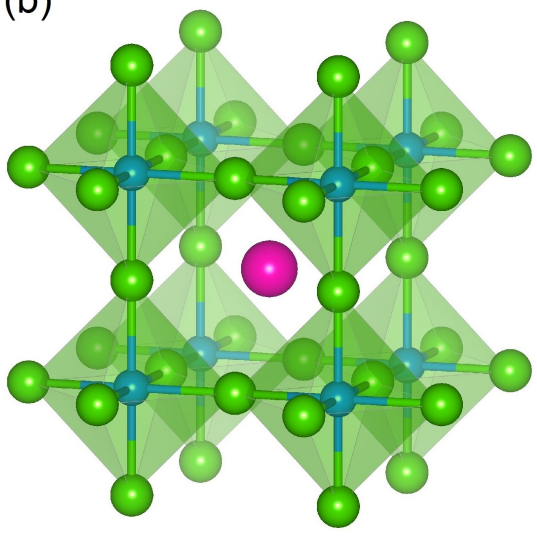

(d)

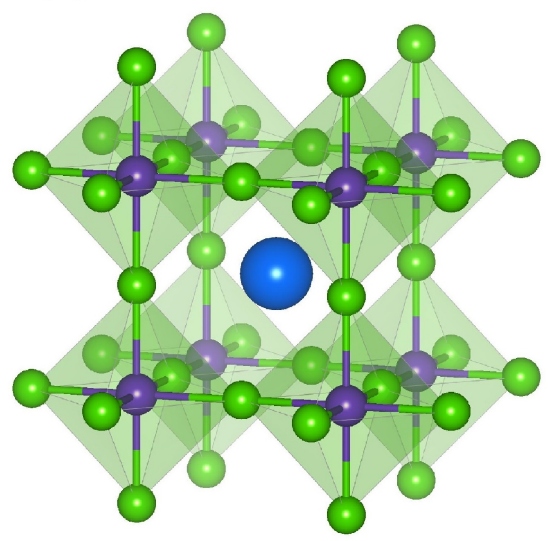

Figure 2.10: Crystal structure of (a) $\mathrm{NaMgF}_{3}$, where red sites are $\mathrm{Na}^{+}$, blue sites are $\mathrm{Mg}^{2+}$, and green sites are $\mathrm{F}^{-}$. (b) $\mathrm{KMgF}_{3}$, where pink sites are $\mathrm{K}^{+}$, blue sites are $\mathrm{Mg}^{2+}$, and green sites are $\mathrm{F}^{-}$. (c) $\mathrm{RbMgF}_{3}$, where dark blue sites are $\mathrm{Rb}^{+}$, blue sites are $\mathrm{Mg}^{2+}$, and green sites are $\mathrm{F}^{-}$. (d) $\mathrm{CsCdF}_{3}$, where blue sites are $\mathrm{Cs}^{+}$, purple sites are $\mathrm{Cd}^{2+}$, and green sites are $\mathrm{F}^{-}$. 


\subsection{Summary}

In this chapter the key aspects of radiation physics, luminescence physics, and materials properties related to this work have been summarised. The interactions of ionising radiation with matter were discussed and related to various medical applications, including the treatment of cancers using radiation therapy. The emergence of luminescence phenomena as a consequence of the quantisation of orbital electron energies was discussed, along with the influence of the crystal field on the energy levels. The various luminescence phenomena relevant to this work were reviewed. Finally, the materials of interest throughout this research, namely the fluoroperovskites $\mathrm{NaMgF}_{3}, \mathrm{KMgF}_{3}, \mathrm{RbMgF}_{3}$, and $\mathrm{CsCdF}_{3}$ were introduced, and their crystal structures and key properties summarised. 


\section{Chapter 3}

\section{Experimental Methods}

A range of experimental techniques and equipment were employed throughout this work. This chapter serves to detail the methods employed and equipment used for the sample syntheses and characterisations. It also briefly introduces the 2D dosimeter system that will be discussed in detail in Chapter 6 .

\subsection{Sample Fabrication}

Numerous samples were synthesised and studied throughout the period of this work. Samples of pure and doped polycrystalline $\mathrm{NaMgF}_{3}, \mathrm{KMgF}_{3}$, and $\mathrm{RbMgF}_{3}$ were synthesised at Victoria University of Wellington via a high-temperature melt and subsequent slow-cooling method. Samples of $\mathrm{CsCdF}_{3}$ were kindly provided by Dr. S. Schweizer, currently at the South Westphalia University of Applied Sciences. These samples were single crystals and were grown via the Bridgman method.

\subsubsection{Polycrystalline Samples}

The majority of the samples discussed in this work were synthesised in polycrystalline form using a high-temperature melt, slow-cooling method that was optimised prior to the commencement of this work [90]. High quality precursor fluorides ( $\geq 99.95 \%)$, e.g. $\mathrm{NaF}$ and $\mathrm{MgF}_{2}$, were powdered and mixed in stoichiometric ratios in a glovebox containing a low-oxygen $(<1 \mathrm{ppm})$, low-humidity $(<10 \mathrm{ppm}) \mathrm{Ar}$ atmosphere. Small fractions of dopant ions were added to the mixtures, also stoichiometrically, as fluorides, e.g. $\mathrm{SmF}_{3}$ for Sm doping. The powder mixtures were placed in glassy carbon crucibles and sealed before being transferred to a second glovebox with a $\mathrm{N}_{2}$ atmosphere and connected to 
an RF induction furnace. The crucibles were then unsealed and placed in a graphite susceptor before being lowered to the centre of the RF furnace tube. The furnace was then isolated from the glovebox and flushed with zero grade Ar for at least 20 minutes. The Ar flow was maintained throughout the remainder of the high temperature synthesis process to maintain the inert atmosphere.

The temperature profiles of the furnace varied slightly between samples, though all had the following characteristics: (1) the mixture was heated to above the melting point of the desired crystal $\left(A B \mathrm{~F}_{3}\right)$ over 1 - 2 hours, (2) the mixture was slowly cooled through the melting point of the crystal over approximately 15 hours, and (3) the mixtures were allowed to naturally cool to room temperature. After cooling the Ar flow was ceased and the samples were retrieved from the furnace.

In all cases this technique produced samples of the desired crystal, as confirmed by Xray diffraction measurements. Sample transparency depended on the species of dopant ions, and the concentration of the dopant ions, though a majority of samples were of sufficient transparency to perform transmission spectroscopy measurements due to the production of macroscopic crystallites that could be preferentially cut from the bulk crystal.

\subsection{Materials Characterisation}

After synthesis, samples were characterised using the various experimental techniques discussed here. In most cases the measurements performed were dependent on the material of interest, such that not all measurements were made for all samples.

\subsubsection{X-ray Diffraction}

$\mathrm{X}$-ray diffraction $(\mathrm{XRD})$ measurements were performed using $\mathrm{Cu}-K \alpha$ radiation $(1.5406$ A) from a PANalytical X'Pert PRO X-ray diffractometer. Powder samples were prepared by hand grinding crystalline samples in a mortar and pestle until a fine powder was obtained. The powdered samples were loaded into sample holders with dimensions 18 $\mathrm{mm}$ by $2 \mathrm{~mm}$ and placed on a rotating sample stage. Data were typically collected for values of $2 \theta$ ranging from $20^{\circ}$ to $90^{\circ}$ with intervals of approximately $0.01^{\circ}$. The data were pre-processed prior to analysis by subtracting background contributions arising from the sample holder, and stripping of the $\mathrm{Cu}-K \alpha_{2}$ contributions.

The experimental diffractograms were compared with those established for each of 
the compounds under study. All host compounds studied in this work, in their single crystal and polycrystalline forms, are well known to the scientific community and have well established crystal structures and XRD patterns. The primary purpose of the XRD characterisations was to investigate phase purity, the presence of residual fluoride precursors, and the influence of dopants on the lattice parameters of the host compound.

\subsubsection{X-ray Irradiation}

Two X-ray sources were employed throughout the course of this work for the purposes of irradiating samples.

Most irradiations were performed using a Philips PW1730 X-ray generator, adapted from a diffractometer for the purposes of direct sample irradiation. The generator was fitted with a tungsten anode and most frequently operated at $40 \mathrm{kVp}$. The X-ray current was varied between $10 \mathrm{~mA}$ and $40 \mathrm{~mA}$ depending on the experiment, in order to adjust the dose rate in a controlled manner. Approximately $1 \mathrm{~mm}$ of Al filtering was placed between the source and sample in all cases. The dose rate of the generator was estimated previously [90] and was approximated to be $1.0 \pm 0.5 \mathrm{Gys}^{-1}$ to the sample surface of a TLD-100 dosimeter located $65 \mathrm{~mm}$ from the source, when operated at $40 \mathrm{kVp}$ and 40 $\mathrm{mA}$. All dose rate calculations for different materials and different configurations were derived from this approximated value, and thus maintained a significant uncertainty.

The X-ray spectrum was also simulated using the SPEKTR 3.0 computational toolkit [91]. The simulated spectrum at $40 \mathrm{kVp}$ is shown in figure 3.1. From this simulation the dose rate in air at the sample surface was approximately $0.5 \mathrm{Gys}^{-1}$, in reasonable agreement with the approximated value for TLD-100.

When wide-field irradiations were required, a Hewlett-Packard 43855A Faxitron was employed. The Faxitron also employs a tungsten tube and was most frequently operated at $40 \mathrm{kVp}$ and with a fixed current of $0.3 \mathrm{~mA}$. Dose rates using this configuration were previously measured to be approximately $0.5 \mathrm{mGys}^{-1}$ [92].

\subsubsection{Optical Transmission}

Optical transmission measurements were made on various samples in order to evaluate band gap energies, to investigate the influence of dopants, and to characterise any radiation-induced optically-active defects. Measurements were made using either a Shimadzu UV-2100 spectrophotometer or a Shimadzu UV-2600 spectrophotometer, where in most cases the latter was employed. The optics of the UV-2600 are shown in fig- 


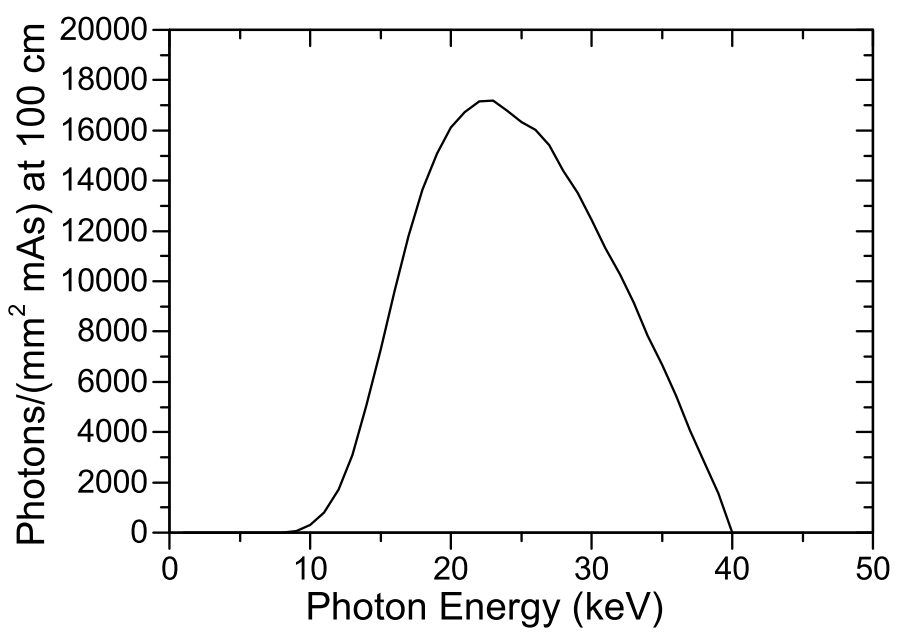

Figure 3.1: X-ray spectrum of the Philips PW1730 X-ray generator operated at $40 \mathrm{kVp}$, $100 \mathrm{~cm}$ from the source, simulated using SPEKTR 3.0 [91].

ure 3.2. Transmission measurements were made over the spectral range $200 \mathrm{~nm}$ to 800 $\mathrm{nm}$ using light emitted by either a halogen lamp (long wavelengths) or deuterium lamp (short wavelengths), where there was a pre-defined point $\lambda=320 \mathrm{~nm}$ below which the system switched to the deuterium lamp. The system used a rotating diffraction grating to generate monochromatic light incident on the sample over the range of wavelengths. In most cases the resolution was $0.5 \mathrm{~nm}$. Transmitted light was detected with a R928 photomultiplier tube (PMT).

Prior to performing optical absorption measurements, samples were cut into pieces approximately $0.5-1.5 \mathrm{~mm}$ thick using a diamond circular saw. The samples were then polished using alumina discs in order to reduce surface scattering. However, in all cases a scattering background was observed that was subtracted when converting from transmission to absorption, via fitting to multiple polynomials. The absorption coefficient, $\alpha$, was calculated per the expression:

$$
\alpha=(1 / d) \ln (100 / T),
$$

where $d$ is the thickness through the sample, and $T$ is the transmittance as a percentage.

\subsubsection{Photoluminescence}

The main characterisation technique employed throughout this work was photoluminescence spectroscopy. PL spectroscopy is often separated into its two primary components, namely photoluminescence emission spectroscopy and photoluminescence exci- 


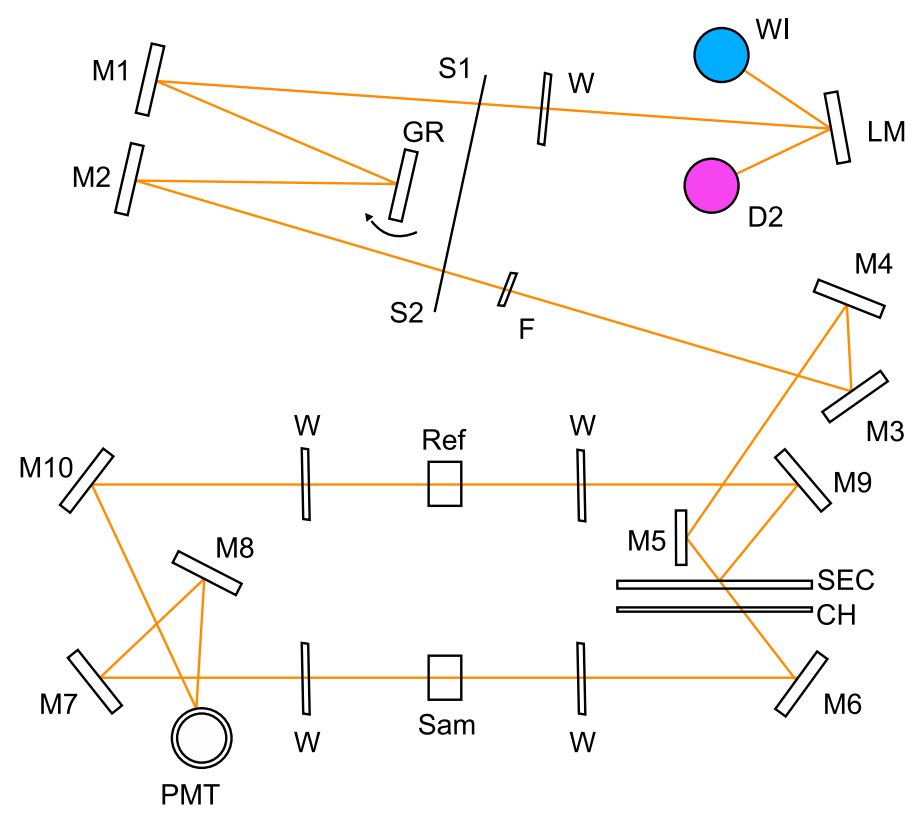

Figure 3.2: Schematic diagram of the Shimadzu UV-2600 optics. The light path is shown in orange. Light emitted from the halogen lamp (WI) or deuterium lamp (D2) is passed through the system depending on the light source switching mirror (LM). Mirrors 1 - 10 (M1-M10) direct the light through the system, where the final wavelength is defined by the position of the grating (GR). The transmission is defined as the percentage of light of a particular wavelength that passes through the sample (Sam) relative to the reference (Ref), which is detected by a PMT (PMT). Other components are slits (S1, S2), window plates $(\mathrm{W})$, a filter $(\mathrm{F})$, a sector mirror $(\mathrm{SEC})$, and a beam chopper $(\mathrm{CH})$. Adapted from Shimadzu UV-2600 documentation [93]. 
tation spectroscopy. In the former case light of a fixed wavelength is used to stimulate the sample while the detected emission wavelength is varied. For the latter case this process is inverted, where the detected emission wavelength is fixed and the excitation wavelength varied.

PL spectra were obtained using a Horiba Jobin-Yvon FluoroLog-3 spectrofluorometer. A schematic illustration of the optics are shown in figure 3.3. The software used to operate the spectrofluorometer was FluorEssence ${ }^{\mathrm{TM}}$.

The excitation source was a xenon discharge lamp capable of exciting the PL of a sample from approximately $250 \mathrm{~nm}$ to $800 \mathrm{~nm}$. Light was directed from the lamp into the excitation chamber that functions as a dual monochromator, where the rotating gratings select the wavelength of interest that is then directed toward the sample. Upon excitation, any light emitted by the sample could be directed one of two ways, depending on the emission wavelength range under study.

For emissions in the visible, the emitted light was directed into the emission chamber that also functioned as a dual monochromator and selected the emission wavelength of interest. The light was directed to a Hamamatsu R928 PMT. The PMT is sensitive from approximately $185 \mathrm{~nm}$ to $900 \mathrm{~nm}$, and thus is well suited to the detection of UV and visible PL emissions.

For emissions in the infrared (IR) an alternative detector was used. PL emissions were instead directed into the Horiba iHR320 attachment that functioned as a single monochromator. The emission intensity at the selected wavelength was detected using an IGA-020-E-LN7 photodiode composed of InGaAs. The photodiode required cooling, achieved by immersing the detector in liquid nitrogen. The spectral range of the detector was approximately $800 \mathrm{~nm}$ to $1550 \mathrm{~nm}$.

A calibrated semiconductor was used to the detect the excitation intensity at all times, such that all spectra were corrected for variations in the lamp emissions over different wavelengths and at different times. In order to avoid harmonic effects, various long pass and band pass filters were employed, depending on the measurement made. The excitation and emission slit widths were variable and set by the software and these were also varied depending on the measurement.

PL lifetimes were measured for some samples using a Cary Eclipse fluorescence spectrometer. This spectrometer used a pulsed Xe lamp with a variable repetition rate and was thus suitable for monitoring longer PL lifetimes above $\sim 10 \mathrm{~ms}$. 


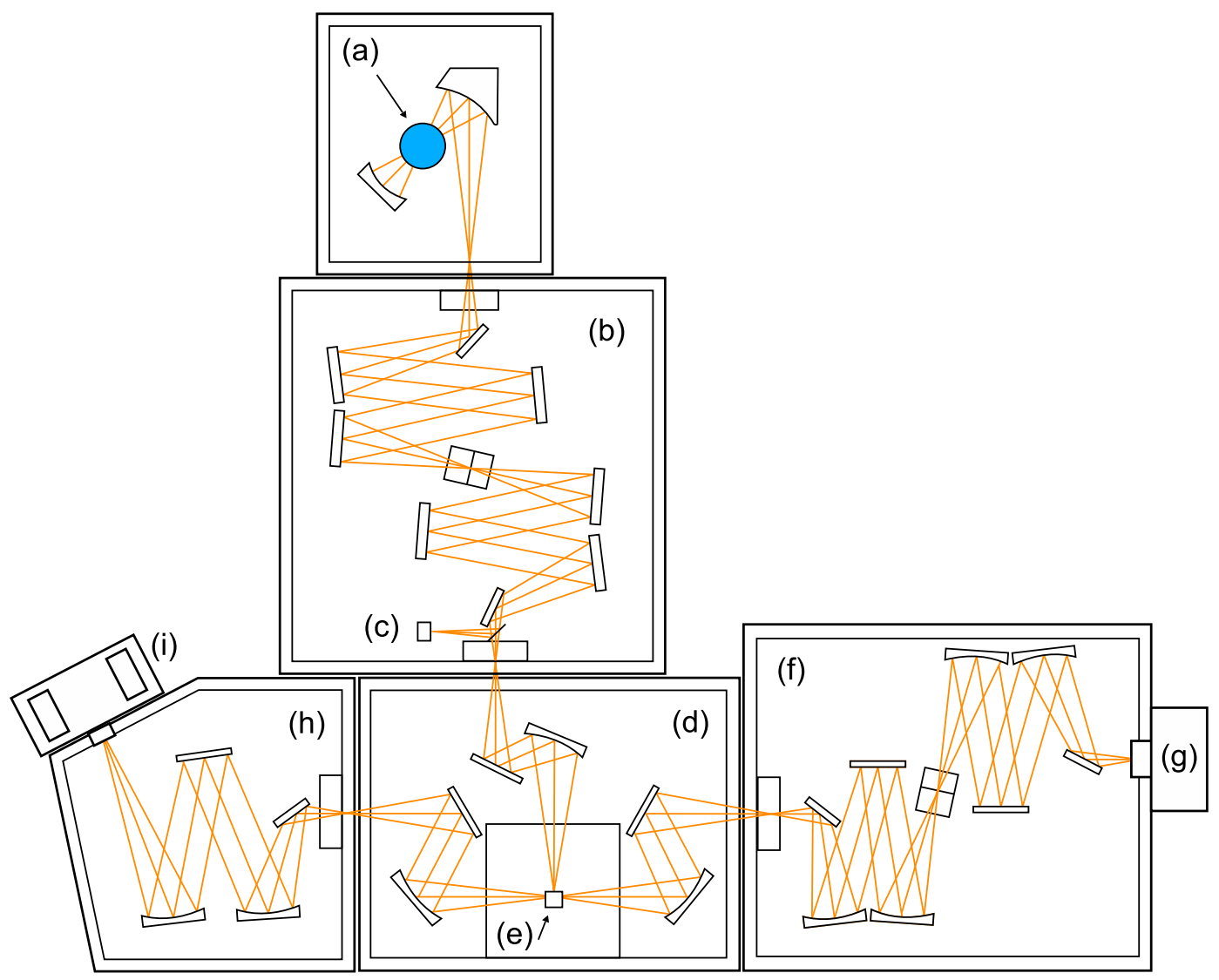

Figure 3.3: Schematic diagram of the Fluorolog-3 optics. The light path is shown in orange. Light is emitted from the xenon lamp (a) and directed into the dual grating monochromator excitation chamber (b). Variations in the excitation intensity are monitored by a semiconducting detector (c). The light is directed into the sample chamber (d) and focused onto the sample (e). The light may then be diverted into the dual grating monochromator emission chamber (f) and to the PMT (g), or alternatively to the single grating IR emission chamber (h) and to an IR detector (i). Adapted from the Fluorolog-3 documentation [94].

\subsubsection{Radioluminescence}

RL measurements were made using a custom built attachment to the Philips PW1730 $\mathrm{X}$-ray generator, such that RL emission spectra were obtained in situ. A schematic of the RL setup is shown in figure 3.4 (a). Small samples approximately $1 \mathrm{~mm}$ thick were attached to the end of an $\mathrm{Al}$ finger that was placed $65 \mathrm{~mm}$ from the X-ray source and tilted $45^{\circ}$. A quartz optical fibre was attached above the sample such that its opening was perpendicular to the X-ray beam. It was ensured that the fibre itself was not exposed to 
the beam in order to avoid any spurious luminescence or dose-dependent effects arising from the fibre. Luminescence was transferred via the fibre to an Ocean Optics USB2000+ CCD spectrometer and monitored as a function of time. For RL measurements the dose rate was taken to be approximately $1 \mathrm{Gys}^{-1}$ and spectra were generally collected over at least 2 hours in order to assess the effects of large radiation doses.

(a)

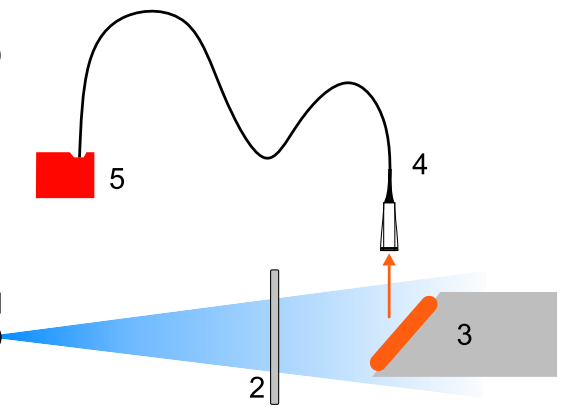

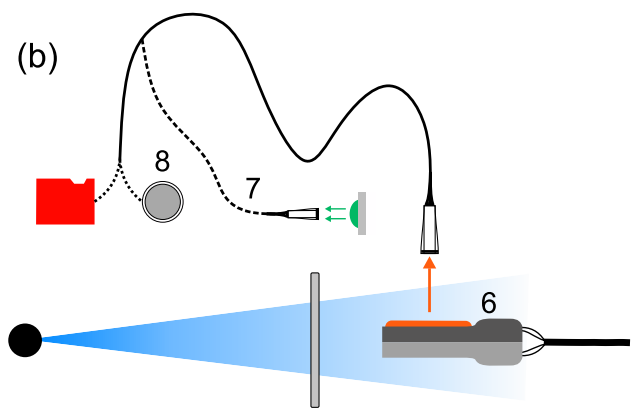

Figure 3.4: Schematic illustrations of the (a) RL setup and (b) TL setup. Numbered components are (1) X-ray source, (2) Al filter, (3) RL sample holder with crystal sample, (4) optical fibre, (5) CCD spectrometer, (6) TL holder with powder sample, (7) optional 50/50 fibre with LED, (8) optional PMT detector.

\subsubsection{Thermoluminescence}

TL spectra were collected via a similar setup to the RL (figure 3.4 (b)) and using the same $\mathrm{X}$-ray generator. However, an alternative sample holder was required in order to heat the samples, which was constructed of two back-to-back PT-100 resistors. The complete apparatus is discussed in detail elsewhere [95] and is summarised here. The PT-100 resistors constitute a TL finger, where one resistor functions to sense temperature while the other functions as a heating element. Samples were ground into a fine powder and adhered to the finger using silicone spray. In all cases a pre-heat was performed in order to evaporate excess silicone oil and homogenise the powder. TL measurements were made post-irradiation and the temperature was controlled using LabVIEW software.

There were two possible means of detecting the TL emissions. One was via the CCD array discussed in Section 3.2.5 that allowed for spectrally resolved emission detection. However, the relatively low sensitivity of the CCD array made some glow curves difficult to obtain with accuracy, and therefore measurements were also made using a PMT where necessary. Detection via PMT did not provide any spectral information, hence, in cases where the PMT was used, the CCD was also used to determine the emission wavelengths of each glow peak. 
TL measurements were also made at low temperatures. This was achieved by flowing dry nitrogen gas through a copper coil that was immersed in a liquid nitrogen bath, that was then directed through the sample holder. For low temperature TL measurements, samples were irradiated at the minimum achievable temperature, then ramped to high temperatures post-irradiation.

In all cases, emissions arising from blackbody radiation were detected. As such, TL glow curves were taken prior to any irradiation to establish the characteristics of the blackbody curve, and the blackbody curve was then subtracted from those curves obtained after irradiation. Additionally, the ceramic PT-100 resistors exhibited weak TL signals that were subtracted from the total signal.

For some compounds, real-time RPL measurements and temperature-dependent PL measurements were made using the same system with some minor adjustments. The optical fibre was swapped for a 50/50 beam split fibre that allowed for simultaneous optical stimulation using an LED while irradiating and/or while heating the sample. Emission spectra were collected via the CCD array. Optical filters were added in order to avoid detecting the stimulating light.

\subsubsection{Magnetisation}

In some samples it was of interest to study intrinsic defects, impurities, and dopant ions via magnetic measurements. Such measurements were performed using a Quantum Design Magnetic Property Measurement System (MPMS) that utilises a superconducting quantum interference device (SQUID) magnetometer. Samples measuring approximately $3 \mathrm{~mm} \times 3 \mathrm{~mm} \times 1 \mathrm{~mm}$ were suspended in plastic straws before being inserted into the MPMS. The magnetisation was measured as a function of temperature between $300 \mathrm{~K}$ and $4 \mathrm{~K}$, where field strengths of either $1 \mathrm{~T}$ or $6 \mathrm{~T}$ were applied.

\subsubsection{Conductivity}

In order to perform radiation-induced conductivity measurements, it was necessary to monitor the electric current passing through a sample during irradiation. This was achieved using custom equipment and the PW1730 X-ray generator. Thin ( 1 mm) samples were cut with large faces (approximately $10 \mathrm{~mm} \times 10 \mathrm{~mm}$ ) and thin wires were attached to both faces using Epotek H20E silver epoxy. The sample was then connected in series with the input of a Keithley 617 Electrometer that monitored the current in realtime and was accurate to approximately $0.05 \mathrm{pA}$. Also connected in series was the voltage 
output of the same electrometer, which was used to apply a variable voltage across the sample. The sample was mounted in the beam line of the X-ray generator while all other electronics were located outside the exposure chamber. In cases of simultaneous electrical and RL detection, an optical fibre was fixed above the sample and perpendicular to the X-ray beam, and the luminescence was collected per the RL experiments (Section $3.2 .5)$.

\subsection{Dosimeter Systems}

Some materials studied throughout this work revealed properties suitable for $2 \mathrm{D}$ dose detection. In order to perform 2D dosimetry, a device was developed that was capable of 2D imaging. OSL was the primary radiation sensing mechanism exploited for imaging. The device used for the 2D characterisations is briefly discussed here, and in more detail in Chapter 6.

\subsubsection{CCD Camera Detection}

The first readout system used LEDs as optical stimulation sources, and a CCD camera for luminescence detection in 2D. The camera was a SBIG STXL-11002 monochromatic CCD camera equipped with a Canon EF $35 \mathrm{~mm}$ f/1.4 lens. The CCD itself was a KAI-11002 with a $9 \mu \mathrm{m}$ pixel size. The CCD was thermoelectrically cooled to $-20^{\circ} \mathrm{C}$ during measurements. Various LEDs were used over the course of the research, where the emission wavelengths were mostly chosen based on the OSL stimulation spectra obtained during the luminescence characterisations. Similarly, various bandpass filters were employed to filter undesirable emissions from the camera aperture, and additional filters were used to narrow the bandwidth of the stimulating LED, where required. 


\section{Chapter 4}

\section{Luminescence of $\mathrm{Mn}^{2+}$ in Fluoroperovskite Hosts}

Transition metals can produce interesting luminescence properties when incorporated into wide band gap compounds, including the fluoroperovskites [27, 30, 73, 77, 96]. These properties have found both real and potential applications in solar cells [97, 98], persistent phosphors [7, 99], optical memory [100], and radiation dosimetry [101], among others.

Manganese $(\mathrm{Mn})$ is a relatively light element $(Z=25)$ that is easily doped into ionic compounds. The Mn atom has the electronic configuration [Ar] $3 d^{5} 4 s^{2}$, though it is commonly incorporated into compounds as the divalent ion $\mathrm{Mn}^{2+}$ with configuration [Ar] $3 d^{5}$. The $3 d^{5}$ valence electrons are largely responsible for the various luminescence features of the ion, including a PL emission that typically peaks between $500 \mathrm{~nm}$ and 700 $\mathrm{nm}[7,99,102]$. Per Section 2.2 .10 , the $\mathrm{Mn}^{2+}$ energy levels are strongly affected by crystal fields and thus the luminescence features vary between different host compounds. The low atomic number of Mn allows for reasonably high dopant concentrations to be incorporated into a host $(\sim 1 \%)$ without negatively affecting the tissue-equivalence of the host.

This chapter focuses on the luminescence properties of $\mathrm{Mn}^{2+}$ in the compounds $\mathrm{NaMgF}_{3}: \mathrm{Mn}, \mathrm{KMgF}_{3}: \mathrm{Mn}, \mathrm{RbMgF}_{3}: \mathrm{Mn}$, and $\mathrm{CsCdF}_{3}: \mathrm{Mn}$. The PL spectra were studied before and after X-ray irradiation. The luminescence properties of each sample were compared and discussed with regard to the relevant crystal structures. In some compounds, defect-dopant complexes were observed post-irradiation. The transition energies of the complexes were experimentally determined and are presented for future reference. Finally, analyses of the charge carrier kinetics and the radiation sensing properties of the 
promising dosimeter material $\mathrm{NaMgF}_{3}: \mathrm{Mn}$ and the potential $\mathrm{UV}$-sensing persistent phosphor $\mathrm{CsCdF}_{3}: \mathrm{Mn}$ are presented.

\subsection{Photoluminescence of the Mn-doped Fluoroper- ovskites}

Samples of polycrystalline $\mathrm{NaMgF}_{3}: 1 \% \mathrm{Mn}, \mathrm{KMgF}_{3}: 1 \% \mathrm{Mn}$, and $\mathrm{RbMgF}_{3}: 1 \% \mathrm{Mn}$ and single crystals of $\mathrm{CsCdF}_{3}: 0.2 \% \mathrm{Mn}$ were initially characterised via PL, before and after X-ray irradiation. In doing so, the basic luminescence properties of the $\mathrm{Mn}^{2+}$ ions, the host lattices, and defects/impurities were evaluated. Furthermore, the effects of ionising radiation on the luminescence properties were investigated. This initial characterisation served to explore the potential range of applications for each compound.

All intraconfigurational $3 d^{5}$ transitions are parity forbidden. The ground state of the free $\mathrm{Mn}^{2+}$ ion is ${ }^{6} \mathrm{~S}$, while the optically accessible excited states are largely limited to the ${ }^{4} \mathrm{G},{ }^{4} \mathrm{D}$, and ${ }^{2}$ I levels. Consequently, a majority of the transitions are also spin forbidden, suggesting that any luminescence from the ion will be weak. However, when the $\mathrm{Mn}^{2+}$ ion is incorporated into a host compound, the interactions of the ion with lattice phonons and the crystal field can result in a partial lifting of spin- and parity-forbidden nature of the transitions and the production of strong luminescence [103].

In all $A B \mathrm{~F}_{3}$ samples studied herein $\mathrm{Mn}^{2+}$ substitutes for the $B^{2+}$ site $(B=\mathrm{Mg}$ or $\mathrm{Cd})$. This was expected based on both the isovalence of the substitution, such that no charge compensation was required, and the ionic radii of each ion. Consider the 6-fold coordination of the $B$ sites forming $B \mathrm{~F}_{6}$ octahedra: the ionic radii of $\mathrm{Mg}^{2+}, \mathrm{Cd}^{2+}$, and $\mathrm{Mn}^{2+}$ (high spin) are $0.72 \AA, 0.95 \AA$, and $0.83 \AA$, respectively [81]. In comparison, the ionic radii of the $A$ sites, for each compound and with the relevant symmetries, are $>1 \AA$ in all cases. The smaller the difference in ionic radii for a dopant and the host atom for which it substitutes, the smaller the lattice distortion required to accommodate the dopant ion, and the greater the chance of successful incorporation.

\subsection{1 $\mathrm{NaMgF}_{3}: \mathrm{Mn}$}

$\mathrm{NaMgF}_{3}$ is an orthorhombic fluoroperovskite with lattice parameters $a=5.36 \AA, b=$ $5.49 \AA, c=7.67 \AA$ [87]. Based on the ionic radii of $\mathrm{Mg}^{2+}$ and $\mathrm{Mn}^{2+}$ in $O_{h}$ symmetry we expected the substitution of $\mathrm{Mn}^{2+}$ for the $\mathrm{Mg}^{2+}$ sites. The $\mathrm{MgF}_{6}$ octahedra in $\mathrm{NaMgF}_{3}$ are corner-sharing and equivalent and therefore only a single $\mathrm{Mn}^{2+}$ site was expected. 

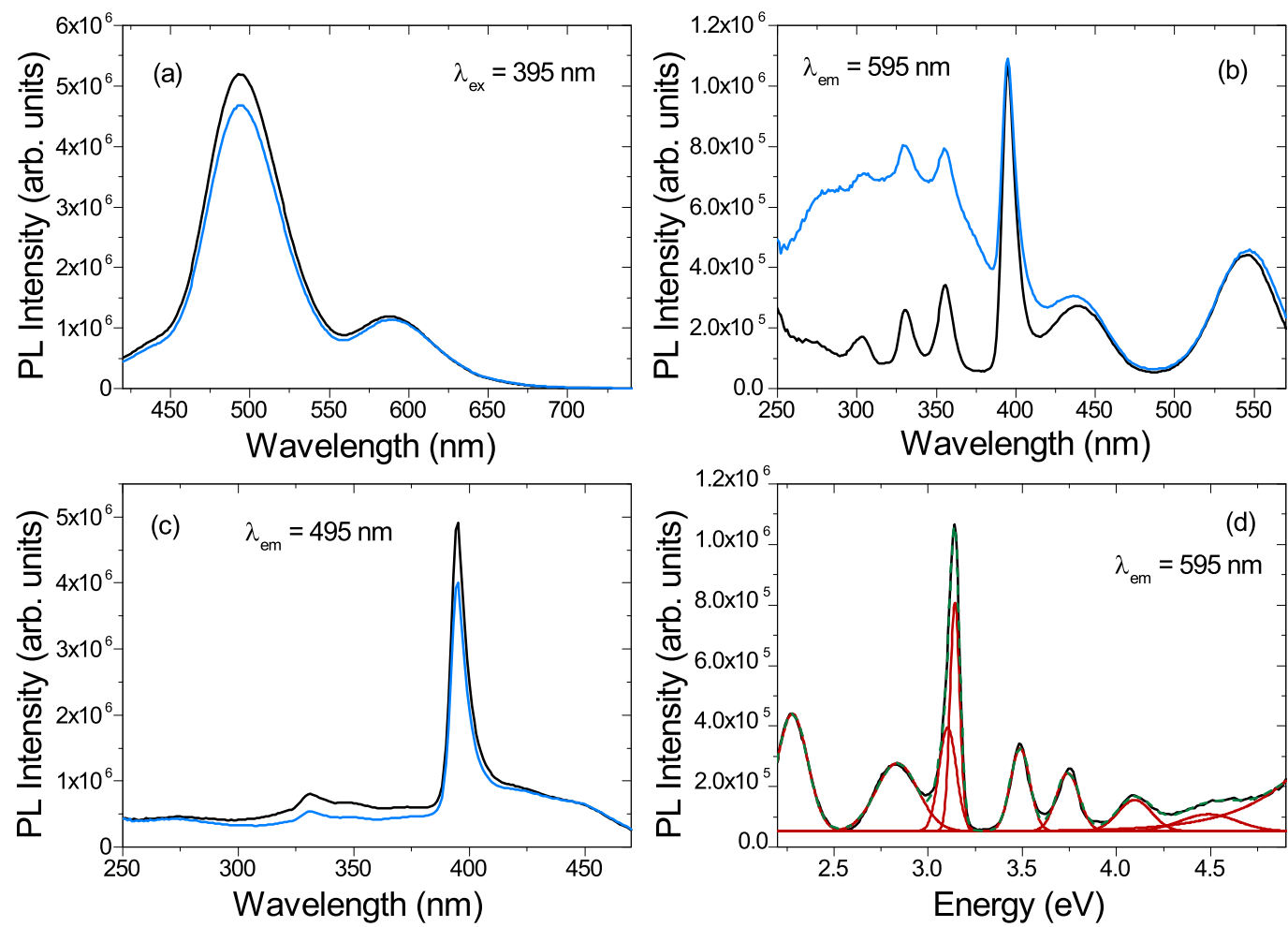

Figure 4.1: PL spectra of $\mathrm{NaMgF}_{3}: \mathrm{Mn}$ before (black) and after (blue) an X-ray dose of 360 Gy. (a) PL emission spectra where $\lambda_{\text {ex }}=395 \mathrm{~nm}$. (b) PL excitation spectra where $\lambda_{\text {em }}=595 \mathrm{~nm}$. (c) PL excitation spectra where $\lambda_{\mathrm{em}}=495 \mathrm{~nm}$. (d) PL excitation spectrum where $\lambda_{\mathrm{em}}=595 \mathrm{~nm}$. Red lines are Gaussian fits to the data. The green dashed line is the cumulative fit. 
The PL spectra of $\mathrm{Mn}^{2+}$ in $\mathrm{NaMgF}_{3}: 1 \% \mathrm{Mn}$ are shown in figure 4.1. When the sample was excited at $\lambda_{\mathrm{ex}}=395 \mathrm{~nm}$ two broad emissions were observed, peaking at $495 \mathrm{~nm}$ and $590 \mathrm{~nm}$ (figure 4.1 (a)). Both emissions appeared only in the Mn-doped sample and the broad bands are characteristic of crystal-field-affected $3 d \rightarrow 3 d$ transitions [7, 99]. The additional component at wavelengths $<450 \mathrm{~nm}$ was likely due to the presence of trace oxygen [27, 104]. After a high X-ray dose (360 Gy) the $495 \mathrm{~nm}$ peak decreased by $10 \%$ and no new luminescence centres were observed.

The emission at $590 \mathrm{~nm}$ is attributed to the ${ }^{4} \mathrm{~T}_{1}(\mathrm{G}) \rightarrow{ }^{6} \mathrm{~A}_{1}(\mathrm{~S})$ transition of $\mathrm{Mn}^{2+}$, where the ion occupies an octahedral crystal field in a high spin configuration. The excitation spectrum for the peak is shown in figure 4.1 (b), where $\lambda_{\mathrm{em}}=595 \mathrm{~nm}$. The multiple excitations observed are consistent with those expected for $\mathrm{Mn}^{2+}$ in $O_{h}$ symmetry, where the crystal field acts to split the free ion levels (table 4.1) [65]. After the X-ray dose the excitation spectrum was unaffected at long wavelengths and a broad band was superimposed at short wavelengths. The background appeared due to the creation of radiation-induced defects, including F-centres, that could be optically stimulated into producing OSL via $\mathrm{Mn}^{2+}[42]$.

The excitation spectrum for the additional $\mathrm{Mn}^{2+}$-related site, where $\lambda_{\mathrm{em}}=495 \mathrm{~nm}$, is shown in figure 4.1 (c). Two clear peaks were observed at $395 \mathrm{~nm}$ and $331 \mathrm{~nm}$, both of which were also seen for the $590 \mathrm{~nm}$ emission. The $495 \mathrm{~nm}$ site is attributed to the same ${ }^{4} \mathrm{~T}_{1}(\mathrm{G}) \rightarrow{ }^{6} \mathrm{~A}_{1}(\mathrm{~S})$ transition of $\mathrm{Mn}^{2+}$, though for $\mathrm{Mn}^{2+}$ in sites that were highly distorted relative to the ideal $O_{h}$ sites. This is consistent with the expected crystal field splitting for $3 d^{5}$ electrons in $O_{h}$ symmetry. Considering the relevant Tanabe-Sugano diagram (figure $2.8(\mathrm{~b})$ ), it is apparent that the overlapping ${ }^{4} \mathrm{~A}_{1}(\mathrm{G})$ and ${ }^{4} \mathrm{E}(\mathrm{G})$ levels, and the ${ }^{4} \mathrm{E}(\mathrm{D})$ level, remain approximately constant in energy relative to the ${ }^{6} \mathrm{~A}_{1}(\mathrm{~S})$ ground state for increasing values of $10 D q$. In contrast, a majority of the other energy levels change rapidly with $10 D q$. Thus, when $\mathrm{Mn}^{2+}$ ions occupy highly distorted sites, as characterised by a range of $10 D q$ values, peak broadening will occur for all excitations other than those to the ${ }^{4} \mathrm{~A}_{1},{ }^{4} \mathrm{E}(\mathrm{G})$ and ${ }^{4} \mathrm{E}(\mathrm{D})$ levels. Consequently, we observed only those unaffected transitions clearly in the excitation spectrum for the distorted site. Henceforth, the $\mathrm{Mn}^{2+}$ sites that produce emissions at $495 \mathrm{~nm}$ and $590 \mathrm{~nm}$ will be referred to as distorted and nondistorted $\mathrm{Mn}^{2+}$, respectively. X-ray irradiation slightly reduced the excitation intensities for the distorted site per the emission spectrum.

The crystal field parameters, $10 D q$ and $B$, of the non-distorted $\mathrm{Mn}^{2+}$ were calculated by intially fitting the excitation spectrum to a sum of Gaussian components (figure 4.1 (d)) such that the transition energies from the ${ }^{6} \mathrm{~A}_{1}(\mathrm{~S})$ ground state to the various excited 
states were obtained. Subsequently, the ratios of the fitted transition energies were fitted to the ratios obtained from the $3 d^{5}$ Tanabe-Sugano diagram, such that $B$ was determined, and $10 D q$ calculated. The calculated values were $10 D q=9270 \pm 123 \mathrm{~cm}^{-1}$ and $B=781$ $\pm 10 \mathrm{~cm}^{-1}$.
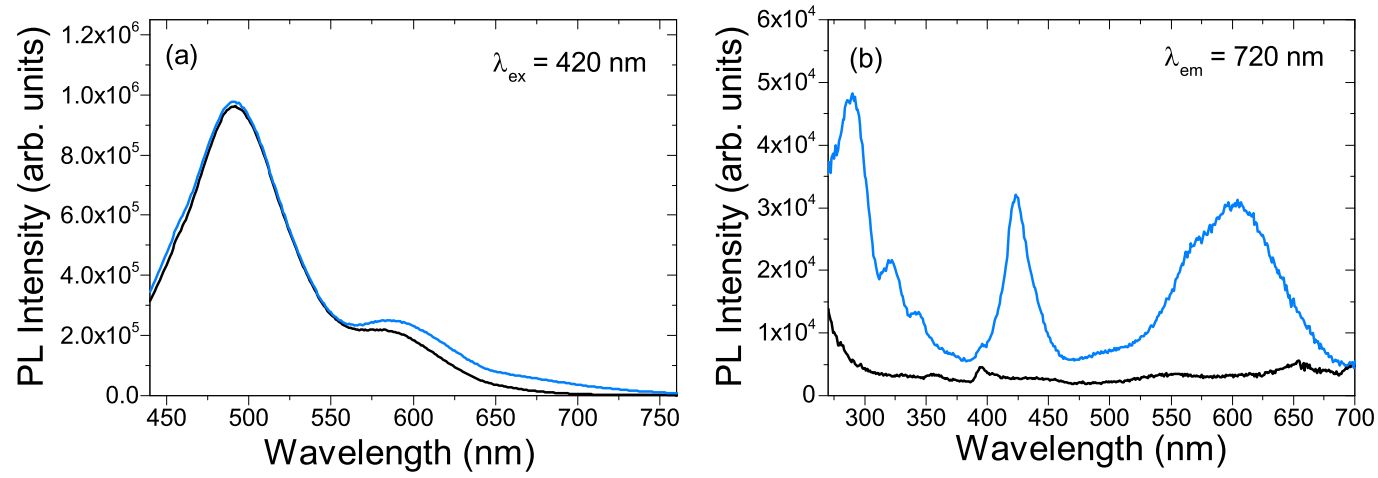

Figure 4.2: $\mathrm{F} / \mathrm{Mn}$ complex PL in $\mathrm{NaMgF}_{3}: \mathrm{Mn}$ before (black) and after (blue) an X-ray dose of 360 Gy. (a) PL emission spectra where $\lambda_{\text {ex }}=420 \mathrm{~nm}$. (b) PL excitation spectra where $\lambda_{\mathrm{em}}=720 \mathrm{~nm}$.

Radiation-induced luminescence centres were detected in the sample via careful selection of the emission and excitation wavelengths. When the excitation wavelength was $\lambda_{\text {ex }}=420 \mathrm{~nm}$ (figure 4.2 (a)) emissions due to both the distorted and non-distorted $\mathrm{Mn}^{2+}$ sites were observed. After an X-ray dose of $360 \mathrm{~Gy}$, a new emission component appeared at wavelengths $>650 \mathrm{~nm}$. The production of new luminescence centres was made clear upon taking the excitation spectrum of the radiation-induced component, where $\lambda_{\mathrm{em}}=$ $720 \mathrm{~nm}$ (figure 4.2 (b)). Prior to irradiation, only weak $\mathrm{Mn}^{2+}$ excitations were observed. After irradiation, new excitations were observed peaking at approximately $290 \mathrm{~nm}, 322$ $\mathrm{nm}, 344 \mathrm{~nm}, 423 \mathrm{~nm}$, and $602 \mathrm{~nm}$. These peaks did not correlate with the energies of the $\mathrm{Mn}^{2+}$ excitations, though bear a strong resemblance in terms of overall line structure and broadening. The excitations were attributed to F-centre/Mn complexes. These defect-dopant structures will be discussed in detail in Section 4.2.

\subsection{2 $\mathrm{KMgF}_{3}: \mathrm{Mn}$}

$\mathrm{KMgF}_{3}$ is a cubic fluoroperovskite with lattice parameter $a=3.99 \AA$ [62]. All $\mathrm{MgF}_{6}$ octahedra are equivalent and hence a single $\mathrm{Mn}^{2+}$ site was expected.

The PL spectra of $\mathrm{Mn}^{2+}$ in $\mathrm{KMgF}_{3}: 1 \% \mathrm{Mn}$ are shown in figure 4.3. When the sample was excited at $\lambda_{\text {ex }}=395 \mathrm{~nm}$ (figure 4.3 (a)) two emissions were observed, peaking at 

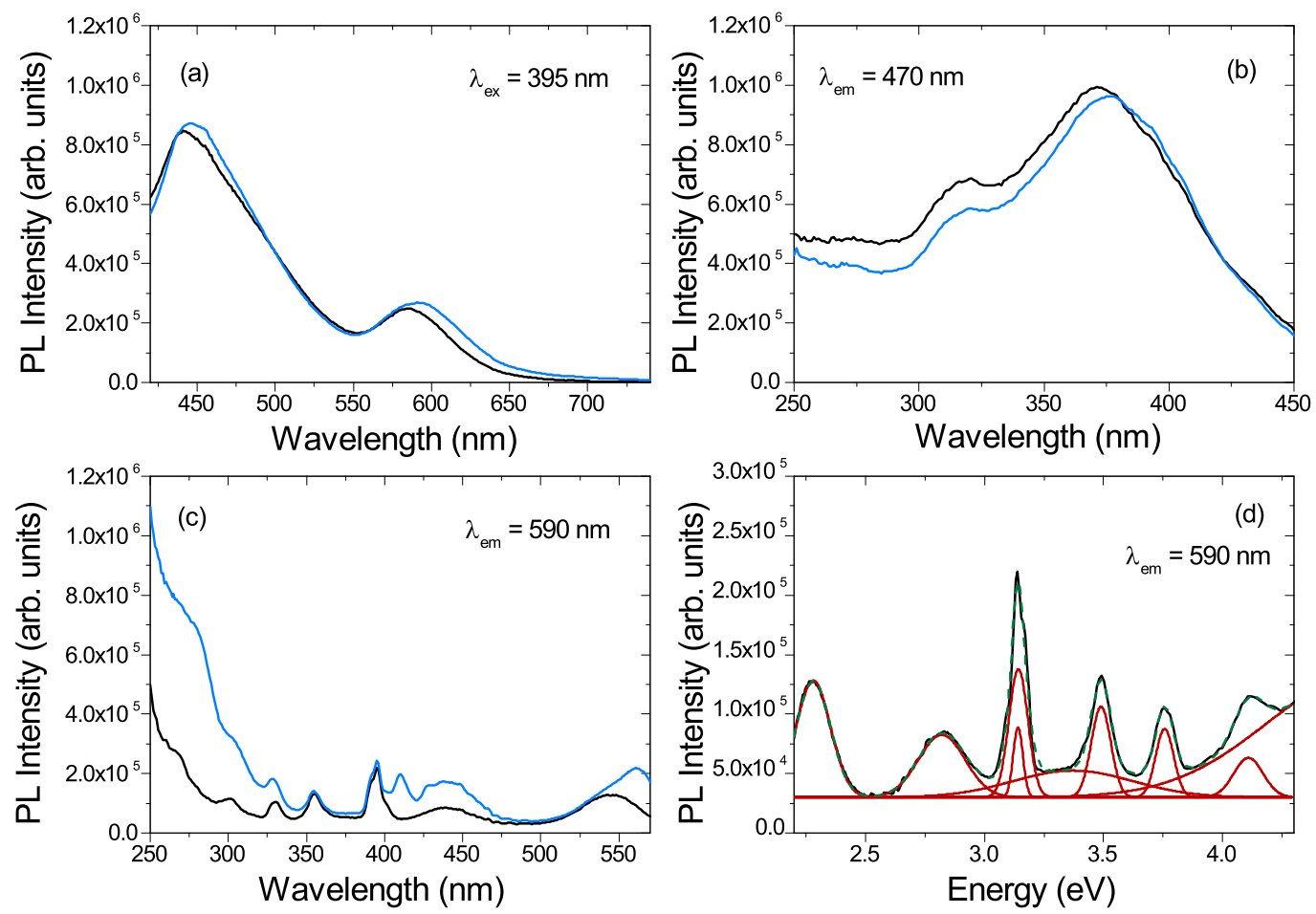

Figure 4.3: Basic PL properties of $\mathrm{KMgF}_{3}: \mathrm{Mn}$ before (black) and after (blue) an X-ray dose of $360 \mathrm{~Gy}$. (a) PL emission spectra where $\lambda_{\text {ex }}=395 \mathrm{~nm}$. (b) PL excitation spectra where $\lambda_{\mathrm{em}}=470 \mathrm{~nm}$. (c) PL excitation spectra where $\lambda_{\mathrm{em}}=590 \mathrm{~nm}$. (d) PL excitation spectrum where $\lambda_{\mathrm{em}}=590 \mathrm{~nm}$. Red lines are Gaussian fits to the data. The green dashed line is the cumulative fit. 
$445 \mathrm{~nm}$ and $585 \mathrm{~nm}$. The $585 \mathrm{~nm}$ emission was due to the ${ }^{4} \mathrm{~T}_{1}(\mathrm{G}) \rightarrow{ }^{6} \mathrm{~A}_{1}(\mathrm{~S})$ transition of $\mathrm{Mn}^{2+}$. The $445 \mathrm{~nm}$ emission was due to the presence of trace oxygen in the host lattice $[27,62,104]$. The excitation spectra for the oxygen-related impurity centres are shown in figure 4.3 (b), where components at approximately $275 \mathrm{~nm}, 320 \mathrm{~nm}$, and 370 $\mathrm{nm}$ are clear. A high X-ray dose of $360 \mathrm{~Gy}$ did not significantly affect either spectrum, though a slight increase in the emission at long wavelengths $>600 \mathrm{~nm}$ was observed that correlated with a slight decrease in the excitation intensities between $250 \mathrm{~nm}$ and 380 nm.

The excitation spectrum for the $\mathrm{Mn}^{2+}$ emission where $\lambda_{\mathrm{em}}=590 \mathrm{~nm}$ is shown in figure 4.3 (c). Multiple excitations were apparent and attributed to transitions from the $\mathrm{Mn}^{2+}$ ground state to the various available excited states. The spectrum was significantly altered by the X-ray dose, where a broad background was superimposed at short wavelengths $<350 \mathrm{~nm}$ and additional excitation components were observed at approximately $410 \mathrm{~nm}, 440 \mathrm{~nm}$, and $560 \mathrm{~nm}$. The short wavelength background was due to the radiation-induced production of F-type centres that could be stimulated into producing OSL via $\mathrm{Mn}^{2+}$ sites.

The $\mathrm{Mn}^{2+}$ excitation spectrum was fitted to a sum of Gaussians in order to evaluate the $O_{h}$ crystal field parameters (figure 4.3 (d)). These were calculated to be $10 D q=9221$ $\pm 139 \mathrm{~cm}^{-1}$ and $B=777 \pm 12 \mathrm{~cm}^{-1}$.

In order to better investigate the radiation-induced luminescence features additional PL spectra were obtained using alternative excitation and emission wavelengths. Figure 4.4 (a) shows the emission spectra where $\lambda_{\mathrm{ex}}=409 \mathrm{~nm}$ before and after irradiation. Prior to irradiation only the oxygen-related emission was clear. After irradiation new emissions were observed peaking at $615 \mathrm{~nm}$ and at wavelengths $>680 \mathrm{~nm}$. There was an additional weak component that peaked near $570 \mathrm{~nm}$ that was due to radiation-induced $\mathrm{F}_{2}$-centres $[105,106]$. The excitation spectra where $\lambda_{\mathrm{em}}=620 \mathrm{~nm}$ are shown in figure 4.4 (b). As the $615 \mathrm{~nm}$ emission overlapped the $\mathrm{Mn}^{2+}$ emission band, only $\mathrm{Mn}^{2+}$ excitations were observed prior to irradiation. After irradiation, new excitation bands appeared that were significantly more intense than those of $\mathrm{Mn}^{2+}$. Clear peaks were observed at 280 $\mathrm{nm}, 307 \mathrm{~nm}, 327 \mathrm{~nm}, 409 \mathrm{~nm}, 427 \mathrm{~nm}$, and $563 \mathrm{~nm}$. The peaks were shifted from those of $\mathrm{Mn}^{2+}$, indicating that new luminescence centres were formed.

Figure 4.4 (c) shows the emission spectra where $\lambda_{\mathrm{ex}}=422 \mathrm{~nm}$ before and after irradiation. Similar emissions were observed per the previously discussed spectra, though where the emission peaking at $695 \mathrm{~nm}$ was more apparent. The excitation spectra where $\lambda_{\text {em }}=700 \mathrm{~nm}$ (figure 4.4 (c)) showed clear excitations only after irradiation with well- 

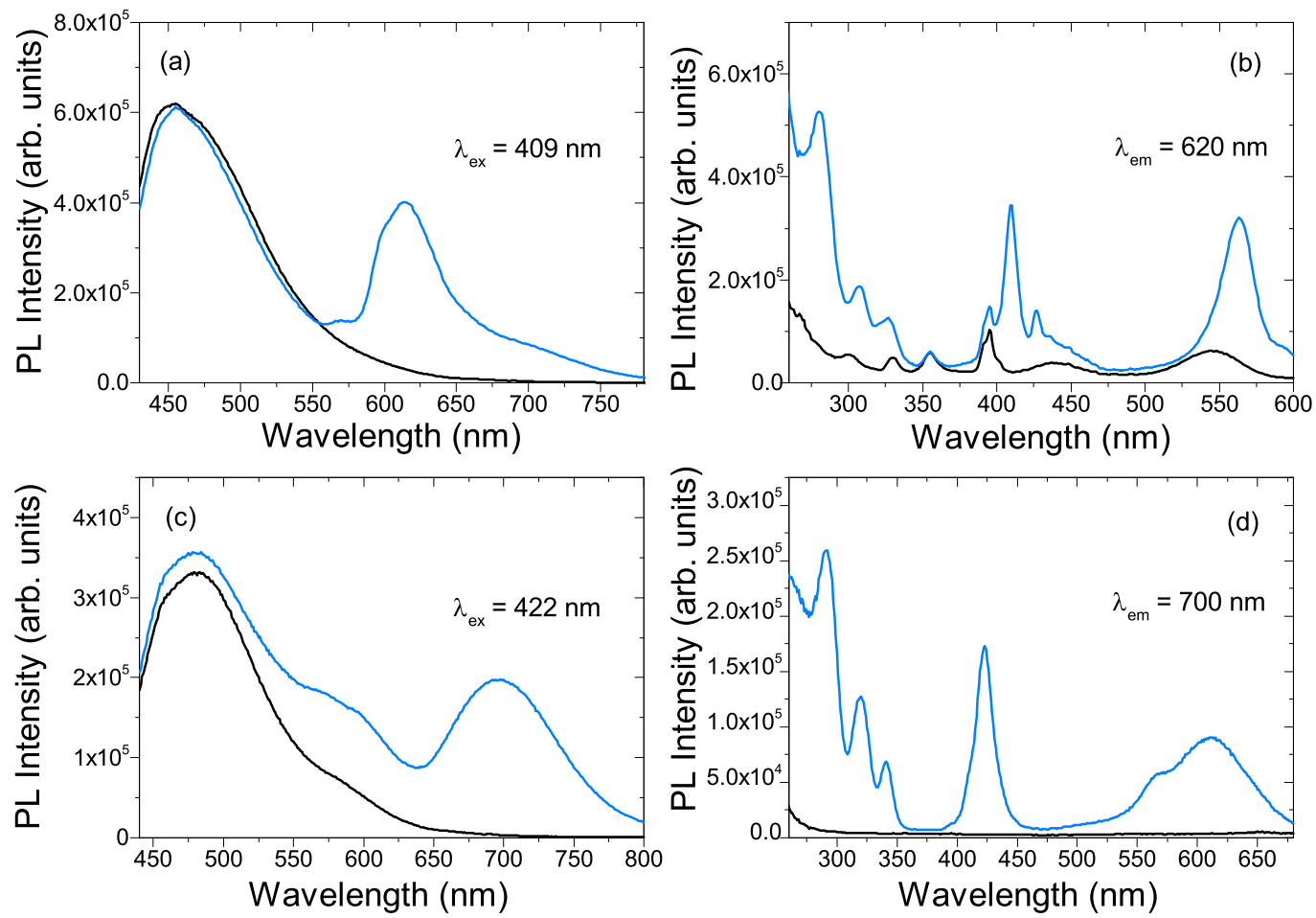

Figure 4.4: $\mathrm{F} / \mathrm{Mn}$ complex $\mathrm{PL}$ in $\mathrm{KMgF}_{3}: \mathrm{Mn}$ before (black) and after (blue) an X-ray dose of $360 \mathrm{~Gy}$. (a) PL emission spectra where $\lambda_{\mathrm{ex}}=409 \mathrm{~nm}$. (b) PL excitation spectra where $\lambda_{\mathrm{em}}=620 \mathrm{~nm}$. (c) PL emission spectra where $\lambda_{\mathrm{ex}}=422 \mathrm{~nm}$. (d) PL excitation spectra where $\lambda_{\mathrm{em}}=700 \mathrm{~nm}$. 
defined peaks at $292 \mathrm{~nm}, 320 \mathrm{~nm}, 341 \mathrm{~nm}, 423 \mathrm{~nm}$, and $613 \mathrm{~nm}$. These excitations were shifted from those of $\mathrm{Mn}^{2+}$, and also those previously discussed for $\lambda_{\mathrm{em}}=620 \mathrm{~nm}$. Therefore, at least two unique radiation-induced luminescent centres could be formed in $\mathrm{KMgF}_{3}: \mathrm{Mn}$. Both were attributed to different types of F-centre/Mn complexes, to be discussed in detail in Section 4.2.

\subsection{3 $\mathrm{RbMgF}_{3}: \mathrm{Mn}$}
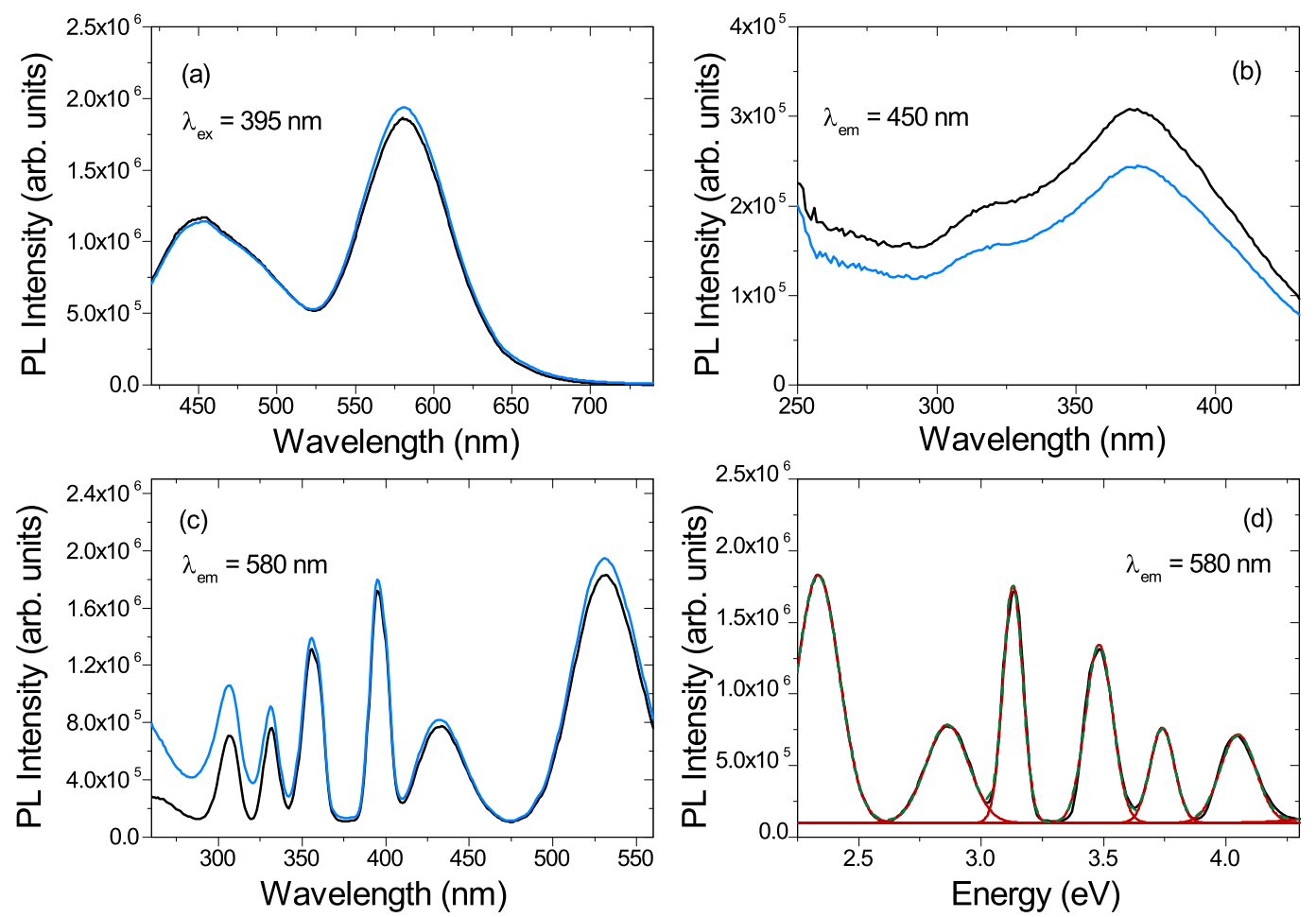

Figure 4.5: Basic PL properties of $\mathrm{RbMgF}_{3}: \mathrm{Mn}$ before (black) and after (blue) an X-ray dose of 360 Gy. (a) PL emission spectra where $\lambda_{\text {ex }}=395 \mathrm{~nm}$. (b) PL excitation spectra where $\lambda_{\mathrm{em}}=450 \mathrm{~nm}$. (c) PL excitation spectra where $\lambda_{\mathrm{em}}=580 \mathrm{~nm}$. (d) (d) PL excitation spectrum where $\lambda_{\mathrm{em}}=580 \mathrm{~nm}$. Red lines are Gaussian fits to the data. The green dashed line is the cumulative fit.

$\mathrm{RbMgF}_{3}$ is a hexagonal fluoroperovskite with lattice parameters $a=5.83 \AA, b=5.83$ $\AA, c=14.20 \AA$ [88]. The lattice contains two non-equivalent $\mathrm{Mg}^{2+}$ sites, where both corner-sharing $\mathrm{MgF}_{6}$ octahedra (labeled $\mathrm{Mg} 1$ ) and face-sharing $\mathrm{MgF}_{6}$ octahedra (labeled $\mathrm{Mg} 2$ ) exist $[88,107]$. We then expect two unique $\mathrm{Mn}^{2+}$ sites upon $\mathrm{Mn}$ substitution, $\mathrm{Mn} 1$ and $\mathrm{Mn} 2$, with different $C_{3 v}$ symmetries. However, the $\mathrm{Mn}^{2+}$ energy levels for each site coincided, and could be readily modeled using an $O_{h}$ crystal field for a single site that 
effectively acts as an average of the two distinct sites.

The PL spectra of $\mathrm{Mn}^{2+}$ in $\mathrm{RbMgF}_{3}: 1 \% \mathrm{Mn}$ are shown in figure 4.5. When the sample was excited at $\lambda_{\text {ex }}=395 \mathrm{~nm}$ (figure 4.5 (a)) two emissions were observed that peaked at $455 \mathrm{~nm}$ and $580 \mathrm{~nm}$. The former emission is attributed to oxygen-related centres and the latter is attributed to the ${ }^{4} \mathrm{~T}_{1}(\mathrm{G}) \rightarrow{ }^{6} \mathrm{~A}_{1}(\mathrm{~S})$ transition of $\mathrm{Mn}^{2+}$. No significant changes were observed after an X-ray dose of $360 \mathrm{~Gy}$. The excitation spectra for the oxygenrelated centres are shown in figure 4.5 (b), with components peaking at approximately $275 \mathrm{~nm}, 315 \mathrm{~nm}$, and $370 \mathrm{~nm}$. The excitation intensities were reduced slightly after the period of irradiation.

The excitation spectra for $\mathrm{Mn}^{2+}$, where $\lambda_{\mathrm{em}}=580 \mathrm{~nm}$, are shown in figure 4.5 (c). The multiple distinct excitations are characteristic of $\mathrm{Mn}^{2+}$ in $O_{h}$ symmetry and were all readily assigned to specific transitions from the ${ }^{6} \mathrm{~A}_{1}(\mathrm{~S})$ ground state. The X-ray irradiation produced an additional broad excitation component at low wavelengths $<350 \mathrm{~nm}$ that had peak wavelengths coincident with the expected F-centre absorption band for $\mathrm{RbMgF}_{3}$ [108].

The $\mathrm{Mn}^{2+}$ excitation spectrum was fitted to a sum of Gaussians and the crystal field parameters were evaluated (figure 4.5 (d)). These were calculated to be $10 D q=8673 \pm$ $154 \mathrm{~cm}^{-1}$ and $B=776 \pm 14 \mathrm{~cm}^{-1}$.
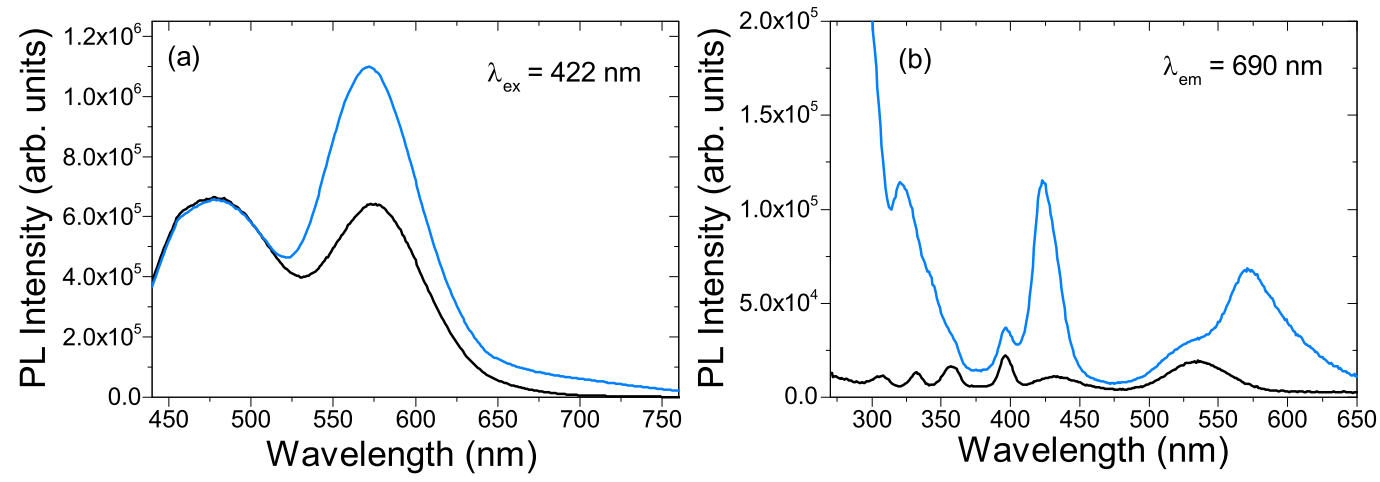

Figure 4.6: F/Mn complex PL in $\mathrm{RbMgF}_{3}: \mathrm{Mn}$ before (black) and after (blue) an X-ray dose of $360 \mathrm{~Gy}$. (a) PL emission spectra where $\lambda_{\mathrm{ex}}=422 \mathrm{~nm}$. (b) PL excitation spectra where $\lambda_{\mathrm{em}}=690 \mathrm{~nm}$.

Increasing the excitation wavelength to $\lambda_{\mathrm{ex}}=422 \mathrm{~nm}$ revealed an additional emission component at wavelengths $>650 \mathrm{~nm}$ that appeared only after irradiation (figure 4.6 (a)). Taking the excitation spectra at $\lambda_{\mathrm{em}}=690 \mathrm{~nm}$ before and after irradiation made clear the presence of radiation-induced luminescence features (figure 4.6 (b)). Prior to irradiation, only the expected $\mathrm{Mn}^{2+}$ excitations were observed. After an X-ray dose of $360 \mathrm{~Gy}$, new 
excitations appeared with clear peaks at $321 \mathrm{~nm}, 423 \mathrm{~nm}$, and $571 \mathrm{~nm}$. The radiationinduced excitations resembled those of $\mathrm{Mn}^{2+}$, though were shifted in regard to both the excitation and the emission energies. The new PL centres were attributed to F-centre/Mn complexes that form during irradiation and will be discussed in Section 4.2.

\subsection{4 $\mathrm{CsCdF}_{3}: \mathrm{Mn}$}

$\mathrm{CsCdF}_{3}$ is a cubic fluoroperovskite with lattice parameter $a=4.47 \AA$ [89]. All $\mathrm{CdF}_{6}$ octahedra are equivalent and hence a single $\mathrm{Mn}^{2+}$ site was expected.

Figure 4.7 (a) shows the PL emission spectra where $\lambda_{\text {ex }}=395 \mathrm{~nm}$ before and after exposure to an X-ray dose of $100 \mathrm{~Gy}$. Two emission components were observed, peaking at $455 \mathrm{~nm}$ and $535 \mathrm{~nm}$. The $455 \mathrm{~nm}$ peak correlated with the oxygen-related defect centres seen for the previously discussed samples and exhibited the same excitation peaks. The $535 \mathrm{~nm}$ peak was attributed to the ${ }^{4} \mathrm{~T}_{1}(\mathrm{G}) \rightarrow{ }^{6} \mathrm{~A}_{1}(\mathrm{~S})$ transition of $\mathrm{Mn}^{2+}$ that has substituted the $\mathrm{Cd}^{2+}$ lattice sites in $O_{h}$ symmetry. The period of X-ray irradiation slightly increased the emission intensities of both sites, though this was likely due to an increase in the $\mathrm{Mn}^{2+}$ emission where the $455 \mathrm{~nm}$ emission increase was due to overlap with the $\mathrm{Mn}^{2+}$ emission. When the excitation wavelength was shifted to $\lambda_{\text {ex }}=260 \mathrm{~nm}$ (figure 4.7 (b)), the $\mathrm{Mn}^{2+}$ emission peak was better resolved, peaking at $540 \mathrm{~nm}$. After X-ray irradiation, the peak emission intensity increased by $\sim 27 \%$, suggesting the radiation-induced creation of defect centres that could produce OSL.

The $\mathrm{Mn}^{2+}$ excitation spectrum is shown in figure 4.7 (c) where $\lambda_{\mathrm{em}}=540 \mathrm{~nm}$. Prior to irradiation the expected $\mathrm{Mn}^{2+}$ excitations from the ${ }^{6} \mathrm{~A}_{1}(\mathrm{~S})$ ground state to the various crystal field split ${ }^{4} \mathrm{G}$ and ${ }^{4} \mathrm{D}$ excited states were apparent. In contrast to the previously discussed samples, there was an additional component for low wavelengths $<300 \mathrm{~nm}$ that was over an order of magnitude more intense than the $\mathrm{Mn}^{2+}$ excitations. The peak of this component could not be resolved by the PL spectrometer as it occurred below $250 \mathrm{~nm}$. Such an intense and broad excitation could be caused by several phenomena. Excitation into the $\mathrm{Mn}^{2+} 4 s$ band (i.e. a $3 d^{5} \rightarrow 3 d^{4} 4 s^{1}$ transition) is parity allowed and would have a greater excitation intensity than the parity forbidden intraconfigurational $3 d$ transitions [109]. However, the $3 d \rightarrow 4 s$ absorptions typically occur at higher energies $(\sim 7 \mathrm{eV})$ in similar materials [109]. The excitation could be due to cross-band gap absorption, where the stimulation at $260 \mathrm{~nm}(4.8 \mathrm{eV})$ is close to the expected band gap $\varepsilon_{g}=5.5 \mathrm{eV}$ [86]. More likely, a charge transfer transition may be responsible, where an electron is either transferred from the valence band to $\mathrm{Mn}^{2+}$ prior to radiative relaxation, or from $\mathrm{Mn}^{2+}$ to the conduction band [110]. Post-irradiation, a broad background 

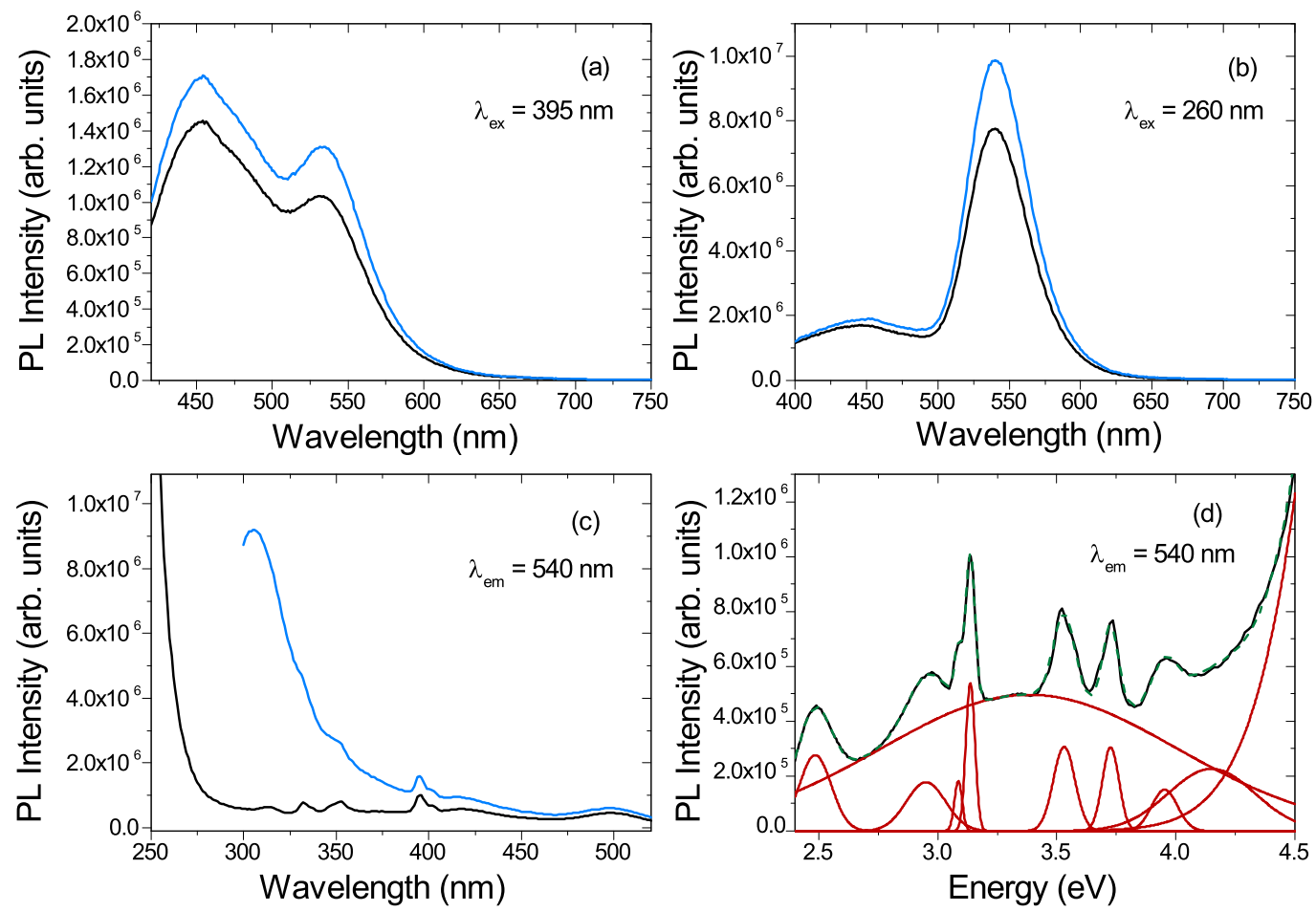

Figure 4.7: Basic PL properties of $\mathrm{CsCdF}_{3}: \mathrm{Mn}$ before (black) and after (blue) an X-ray dose of $100 \mathrm{~Gy}$. (a) PL emission spectra where $\lambda_{\text {ex }}=395 \mathrm{~nm}$. (b) PL emission spectra where $\lambda_{\text {ex }}=260 \mathrm{~nm}$. (c) PL excitation spectra where $\lambda_{\text {em }}=540 \mathrm{~nm}$. (d) PL excitation spectrum where $\lambda_{\mathrm{em}}=540 \mathrm{~nm}$. Red lines are Gaussian fits to the data. The green dashed line is the cumulative fit. 
was superimposed that peaked at approximately $305 \mathrm{~nm}(4.1 \mathrm{eV})$ coinciding with the expected F-centre absorption [86].

The $\mathrm{Mn}^{2+}$ excitation spectrum was fitted to a sum of Gaussians (figure $4.5(\mathrm{~d})$ ). The crystal field parameters were evaluated and calculated to be $10 D q=7020 \pm 120 \mathrm{~cm}^{-1}$ and $B=773 \pm 13 \mathrm{~cm}^{-1}$.

\subsubsection{Comparison and Summary}

The PL properties of $\mathrm{Mn}^{2+}$ doped $\mathrm{NaMgF}_{3}, \mathrm{KMgF}_{3}, \mathrm{RbMgF}_{3}$, and $\mathrm{CsCdF}_{3}$ were presented. The crystal field splitting parameters $10 D q$ and $B$, the PL emission energies of the $\mathrm{Mn}^{2+}$ ${ }^{4} \mathrm{~T}_{1}(\mathrm{G}) \rightarrow{ }^{6} \mathrm{~A}_{1}(\mathrm{~S})$ transitions, and the energies of the first 6 optically-accessible levels of $\mathrm{Mn}^{2+}$ relative to the ground state are presented in table 4.1 for each compound. A number of similarities were observed across the series, due largely to the substitution of $\mathrm{Mn}^{2+}$ for the $B^{2+}$ site in all compounds and the similarities in the crystal structures of each compound. The $A$ site of each compound systematically increased in ionic radius, where the ionic radii for 12 -fold coordinated for $\mathrm{Na}^{+}, \mathrm{K}^{+}, \mathrm{Rb}^{+}$, and $\mathrm{Cs}^{+}$were $1.39 \AA, 1.64$ $\AA, 1.72 \AA$, and $1.88 \AA$, respectively [81]. The primary effect of the larger $A$ sites was on the overall lattice structure per the tolerance factors discussed in Section 2.3. The different structures and lattice parameters produced different $\mathrm{Mg}^{2+}-\mathrm{F}^{-}$and $\mathrm{Cd}^{2+}-\mathrm{F}^{-}$bond lengths, ultimately influencing the $\mathrm{Mn}^{2+}-\mathrm{F}^{-}$lengths and the electric fields produced by the $\mathrm{F}^{-}$ligands.

The $\mathrm{Mg}^{2+}-\mathrm{F}^{-}$bond lengths were calculated to be $1.987 \AA, 1.995 \AA$, and $2.02 \AA$ for $A$ $=\mathrm{Na}, \mathrm{K}$, and $\mathrm{Rb}$, respectively. The $\mathrm{Cd}^{2+}-\mathrm{F}^{-}$bond length was calculated to be $2.235 \AA$. The $\mathrm{Mn}^{2+}-\mathrm{F}^{-}$bond lengths were then approximated by taking the difference between the ionic radii of $\mathrm{Mn}^{2+}$ and the $B^{2+}$ site, calculated as $R_{\mathrm{Mn}-\mathrm{Mg}}=0.11 \AA$ and $R_{\mathrm{Mn}-\mathrm{Cd}}=-0.12$ $\AA$, and adding half the difference to the bond lengths. The $\mathrm{Mn}^{2+}-\mathrm{F}^{-}$bond lengths, $R_{\mathrm{Mn}-\mathrm{F}}$, were approximated to be $2.042 \AA, 2.05 \AA, 2.079 \AA$, and $2.175 \AA$ for $A=\mathrm{Na}, \mathrm{K}, \mathrm{Rb}$, and $\mathrm{Cd}$, respectively.

The crystal field splitting energy, $10 D q$, is proportional to the strength of the local electric field, and the electric field is inversely proportional to the distance of the neighbouring ligands. Consequently, we expect that as the $\mathrm{Mn}^{2+}-\mathrm{F}^{-}$bond lengths increase, $10 D q$ will decrease. This was the case, as illustrated in figure 4.8 , where $10 D q$ is plotted against the estimated bond lengths for each compound. The data were fitted to a power law of the form $10 D q=K R_{\mathrm{Mn}-\mathrm{F}}^{-n}$, where $K=228100 \mathrm{~cm}^{-1}$ and $n=4.48$ were constants. Power law methods for calculating bond lengths from $10 D q$ values, and vice versa, have been proposed in the past using similar $K$ and $n$ values [111]. 

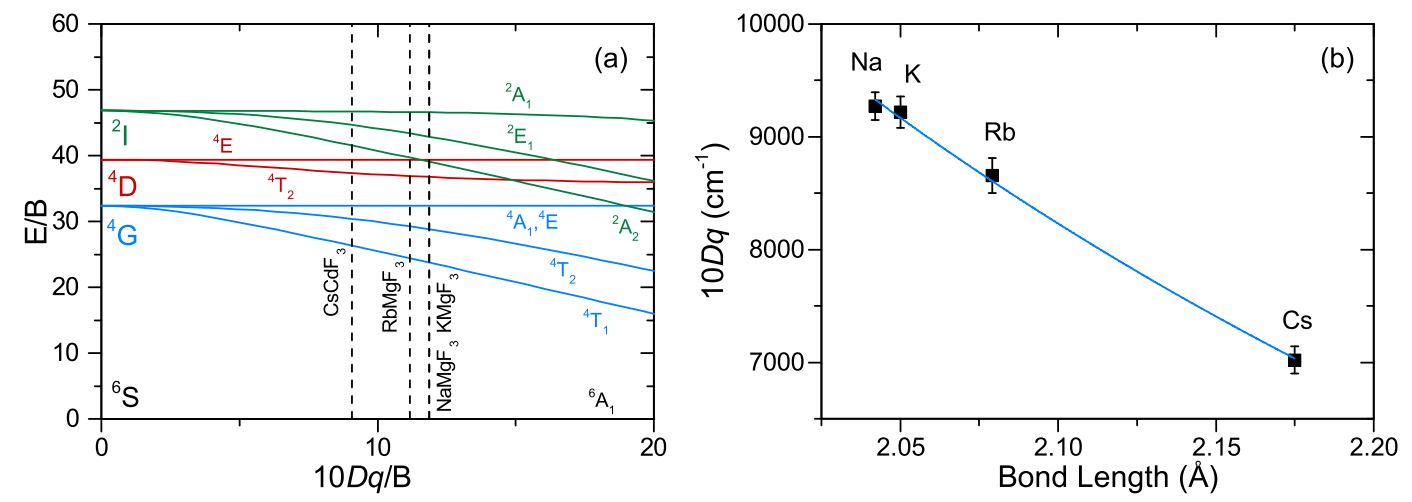

Figure 4.8: (a) Tanabe-Sugano diagram for $3 \mathrm{~d}^{5}$ ions, where the $10 D q / B$ values for $\mathrm{Mn}$ doped $\mathrm{NaMgF}_{3}, \mathrm{KMgF}_{3}, \mathrm{RbMgF}_{3}$, and $\mathrm{CsCdF}_{3}$ are shown. (b) Crystal field splitting energy, $10 D q$, versus the approximate $\mathrm{Mn}-\mathrm{F}$ bond length for $\mathrm{Mn}$-doped $\mathrm{NaMgF}_{3}, \mathrm{KMgF}_{3}$, $\mathrm{RbMgF}_{3}$, and $\mathrm{CsCdF}_{3}$. The blue line is a power law fit to the data.

All samples exhibited a PL emission that peaked at approximately $450 \mathrm{~nm}$ when excited at or near $395 \mathrm{~nm}$. All samples showed similar excitation spectra for the emission. The PL was attributed to oxygen-related defects that incorporated during sample synthesis. Oxygen is a common contaminant in fluoride compounds, where $\mathrm{O}^{2-}$ ions readily substitute $\mathrm{F}^{-}$sites during synthesis [62,112-116]. This substitution requires charge compensation that is likely to occur via the production of $\mathrm{F}^{-}$vacancies that may then trap electrons to form F-centres during irradiation.

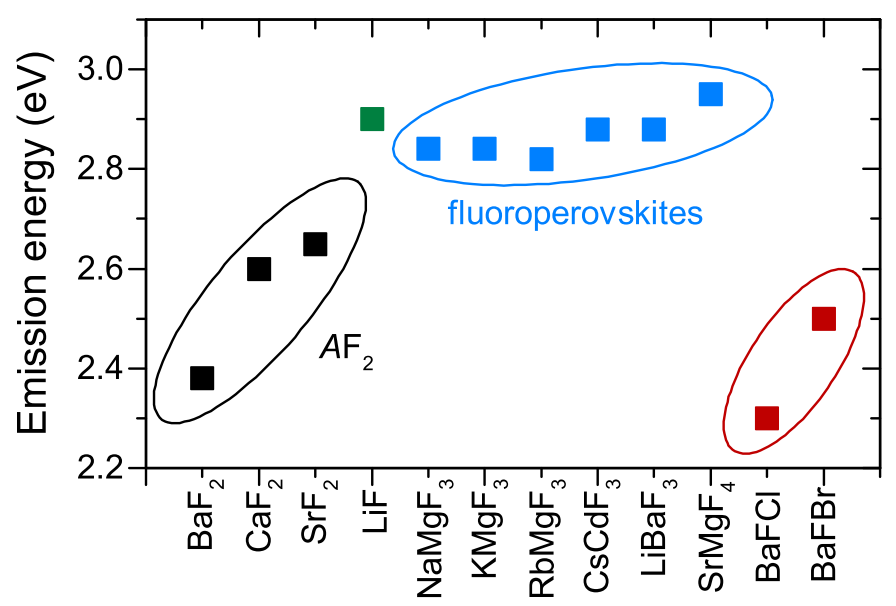

Figure 4.9: Peak emission wavelengths of the oxygen impurity centres in several fluoride compounds.

Oxygen incorporation frequently results in the formation of new luminescence centres in fluorides that emit between $400 \mathrm{~nm}$ and $550 \mathrm{~nm}$ that have historically been at- 
tributed to the formation of $\mathrm{O}^{2-}-\mathrm{V}_{\mathrm{F}}$ dipoles, where $\mathrm{V}_{\mathrm{F}}$ is a charge-compensating fluorine vacancy $[113,117-121]$. The PL emissions of the centres observed in this work resemble the luminescence of said dipoles and are thus attributed to similar oxygen-related centres. The peak PL emission wavelengths of the $\mathrm{O}^{2-}-\mathrm{V}_{\mathrm{F}}$ dipoles in the hosts $\mathrm{BaF}_{2}, \mathrm{CaF}_{2}$, $\mathrm{SrF}_{2}, \mathrm{LiF}, \mathrm{LiBaF}_{3}, \mathrm{SrMgF}_{4}, \mathrm{BaFCl}$, and $\mathrm{BaFBr}$ are shown in figure 4.9, where the values were taken from the literature [113, 117-121]. The peak PL emission wavelengths of the fluoroperovskites studied in this work are also included in the figure.

After X-ray stimulation, all samples exhibited a broad excitation in the UV that peaked near $300 \mathrm{~nm}$ that resulted in $\mathrm{Mn}^{2+}$ emission. These excitations peaked at energies that correlated with the F-centre absorption peaks for each compound, indicating that pre-existing fluorine vacancies trapped electrons during irradiation to form F-centres. The F-centres could be optically stimulated such that the trapped electrons were liberated and recombined to produce $\mathrm{Mn}^{2+}$ luminescence. As such, the broad UV background represented an OSL stimulation curve for all samples. As all samples showed OSL, all samples could theoretically function as OSL dosimeters, where the intensities of the excitation curves, or more likely the integrated intensities of the $\mathrm{Mn}^{2+}$ decay when exciting into the F-centre band, would be proportional to the total X-ray dose that the samples had received. This result is most relevant for the near tissue-equivalent $\mathrm{NaMgF}_{3}: \mathrm{Mn}$ and encouraged further investigation into the OSL behaviour, particularly as a function of radiation dose (Section 4.3).

$\mathrm{NaMgF}_{3}: \mathrm{Mn}$ was unique among the samples in that there were two distinct $\mathrm{Mn}^{2+}$ emissions where only one was expected. The emission at $590 \mathrm{~nm}$ was similar to that seen in the other compounds and was attributed to $\mathrm{Mn}^{2+}$ occupying the standard $\mathrm{Mg}^{2+}$ sites and forming $\mathrm{MnF}_{6}$ octahedra. The higher energy emission at $495 \mathrm{~nm}$ was attributed to highly distorted $\mathrm{Mn}^{2+}$ sites on account of the excitation spectrum, where the ${ }^{4} \mathrm{~A}_{1 \mathrm{~g}}(G)$, ${ }^{4} \mathrm{E}(\mathrm{G})$, and ${ }^{4} \mathrm{E}(\mathrm{D})$ levels showed no line broadening while all other transitions were sufficiently broadened as to be indistinguishable. The mechanism of the site distortion was unclear, though it could be due to a range of adjacent point defects including interstitial fluorines and oxygen impurities. However, the absence of similar distorted sites in the other fluoroperovskites suggests that an alternative mechanism may be responsible for the site distortion, perhaps based on distorted $\mathrm{MgF}_{6}$ octahedra and potentially inequivalent $\mathrm{Mg}^{2+}$ sites.

All $A M g F_{3}$ compounds showed radiation-induced luminescence centres with optical transitions resembling those of the $\mathrm{Mn}^{2+}$ ion and with broad emissions peaking above $600 \mathrm{~nm}$. No such transitions were observed for irradiated $\mathrm{CsCdF}_{3}: \mathrm{Mn}$, possibly due to 


\begin{tabular}{ccccc}
\hline & $\mathrm{NaMgF}_{3}: \mathrm{Mn}$ & $\mathrm{KMgF}_{3}: \mathrm{Mn}$ & $\mathrm{RbMgF}_{3}: \mathrm{Mn}$ & $\mathrm{CsCdF}_{3}: \mathrm{Mn}$ \\
\hline \hline $10 D q\left(\mathrm{~cm}^{-1}\right)$ & $9270 \pm 123$ & $9221 \pm 139$ & $8673 \pm 154$ & $7020 \pm 120$ \\
\hline$B\left(\mathrm{~cm}^{-1}\right)$ & $781 \pm 10$ & $777 \pm 12$ & $776 \pm 14$ & $773 \pm 13$ \\
\hline $\mathrm{Mn}^{2+}$ emission $(\mathrm{eV})$ & 2.10 & 2.12 & 2.14 & 2.30 \\
\hline${ }^{4} \mathrm{~T}_{1}(\mathrm{G})(\mathrm{eV})$ & 2.28 & 2.28 & 2.33 & 2.49 \\
\hline${ }^{4} \mathrm{~T}_{2}(\mathrm{G})(\mathrm{eV})$ & 2.84 & 2.82 & 2.86 & 2.95 \\
\hline${ }^{4} \mathrm{~A}_{1}(\mathrm{G})(\mathrm{eV})$ & 3.10 & 3.14 & 3.13 & 3.09 \\
\hline${ }^{4} \mathrm{E}(\mathrm{G})(\mathrm{eV})$ & 3.14 & 3.14 & 3.13 & 3.14 \\
\hline${ }^{4} \mathrm{~T}_{2}(\mathrm{D})(\mathrm{eV})$ & 3.49 & 3.49 & 3.48 & 3.53 \\
\hline${ }^{4} \mathrm{E}(\mathrm{D})(\mathrm{eV})$ & 3.74 & 3.76 & 3.74 & 3.73 \\
\hline
\end{tabular}

Table 4.1: Summary of the crystal field parameters and $\mathrm{Mn}^{2+}$ transition energies for $\mathrm{NaMgF}_{3}: \mathrm{Mn}, \mathrm{KMgF}_{3}: \mathrm{Mn}, \mathrm{RbMgF}_{3}: \mathrm{Mn}$ and $\mathrm{CsCdF}_{3}: \mathrm{Mn}$.

the increased $\mathrm{Mn}^{2+}-\mathrm{F}^{-}$bond lengths relative to the $A M g F_{3}$ samples. The centres were attributed to F-centre/Mn (F/Mn) complexes. F/Mn complexes have been previously observed in $\mathrm{RbMgF}_{3}: \mathrm{Mn}[57,122-124]$ and $\mathrm{KMgF}_{3}: \mathrm{Mn}$ [26, 75, 76, 125, 126] and also in other fluoride hosts, including $\mathrm{MgF}_{2}: \mathrm{Mn}[75,125,127]$ and $\mathrm{CaF}_{2}: \mathrm{Mn}[128,129]$. Almost all previously published data related to F/Mn complexes were collected several decades ago and the investigations typically focused on very high radiation doses at low temperatures and the analyses of individual spectra and compounds. Radiation-induced luminescence centres represent potential RPL mechanisms that could be used to monitor cumulative radiation doses. Consequently, the F/Mn complexes will be discussed in detail in the forthcoming Section 4.2.

Finally, a broad excitation band was observed at low wavelengths $\sim 250 \mathrm{~nm}$ for the $\mathrm{Mn}^{2+}$ ion in $\mathrm{CsCdF}_{3}: \mathrm{Mn}$ prior to irradiation. Previous experiments demonstrated that the band gap of $\mathrm{CsCdF}_{3}$ is approximately half that of the $A M g F_{3}$ compounds and therefore this band was likely due to some cross-band gap or charge transfer effect that could occur at optical stimulation energies. A thorough investigation into these effects will be presented in Section 4.4. 


\subsection{F-centre/Mn Complexes in Fluoroperovskites}

In the previous section it was demonstrated that the three $A M g F_{3}: M n$ compounds could form new PL-active luminescence centres after X-ray irradiation that appeared as perturbed $\mathrm{Mn}^{2+}$ excitations and emissions. These were attributed to F-centre/Mn (F/Mn) complexes. Similar complexes have been previously observed in the fluoroperovskites $\mathrm{KMgF}_{3}: \mathrm{Mn}[26,75,76,125,126]$ and $\mathrm{RbMgF}_{3}: \mathrm{Mn}[57,122-124]$. Ours is the first observation of such complexes in $\mathrm{NaMgF}_{3}: \mathrm{Mn}$ [42].

F/Mn complexes are not particularly well understood. Theoretical modeling using self-consistent-field (SCF) calculations was attempted for the complexes in $\mathrm{CaF}_{2}: \mathrm{Mn}$ and had difficulty in accurately predicting the transition energies [129]. Structurally, the complexes are believed to be composed of an F-centre $+\mathrm{Mn}^{2+}$ nearest-neighbour pair. The F-centre electron and the $\mathrm{Mn}^{2+} 3 d^{5}$ electrons experience electronic exchange coupling that is thought to at least partially break the spin-forbidden aspect of the $\mathrm{Mn}^{2+}$ transitions. The F/Mn complexes exhibit increased oscillator strengths relative to the $\mathrm{Mn}^{2+}$ ion, where the increase may be by a factor of $10^{3}-10^{5}[75,123,129]$. The increased oscillator strengths result in PL lifetimes reduced by a similar factor [122]. The transition energies of the $\mathrm{F} / \mathrm{Mn}$ complexes are also perturbed relative to the $\mathrm{Mn}^{2+}$ ion, often resulting in well-defined and unique PL excitations and emissions. A schematic illustration of the F-centre $+\mathrm{Mn}^{2+}$ electronic coupling process is shown in figure 4.10.

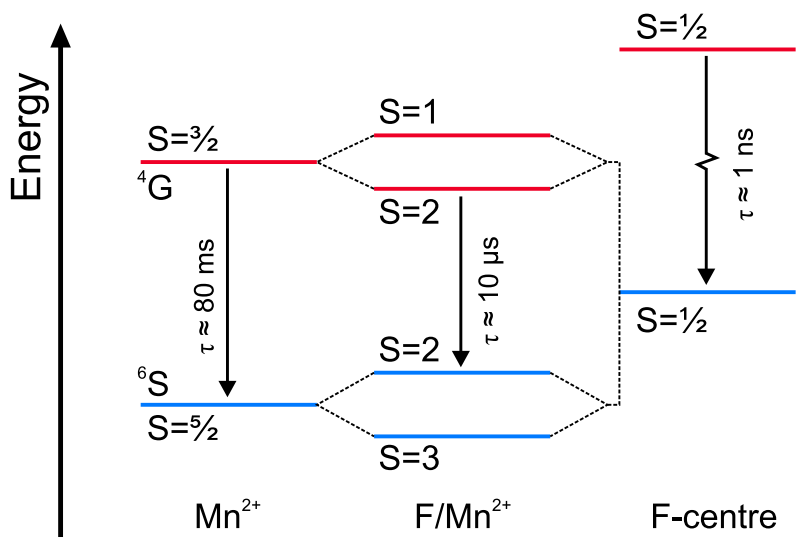

Figure 4.10: Schematic of $\mathrm{Mn}^{2+}$ and F-centre electron coupling, where the effects on the $\mathrm{F} / \mathrm{Mn}$ spin $(\mathrm{S})$ and lifetime $(\tau)$ are shown. Adapted from [122].

As the F/Mn complexes can only form when fluorine vacancies adjacent to a $\mathrm{Mn}^{2+}$ ion capture electrons, the complexes are not seen prior to radiation exposure. As F-centres are readily produced by irradiation, it is possible to use the intensity of the PL arising 
from the F/Mn complexes as a measure of radiation exposure. This constitutes RPL dosimetry. It was expected that the PL could be probed non-destructively by selectively stimulating the material with wavelengths correlated with the lower energy excitation bands, while the complexes could be erased by exciting into the F-centre stimulation band in the UV and consequently emptying the F-centre traps.

The potential applications arising from the existence of $\mathrm{F} / \mathrm{Mn}$ complexes motivated a comprehensive characterisation of the F/Mn complexes in the fluoroperovskites. The intention was that an experimental data set be compiled that will aid in any future theoretical calculations and additionally inspire similar studies in alternative fluoride host compounds, such as LiF:Mn. Established trends across the series of fluoroperovskites should aid in the physical interpretation of data collected for similar compounds. This section is devoted to a study of the PL of the F/Mn complexes in the host compounds $\mathrm{NaMgF}_{3}, \mathrm{KMgF}_{3}$, and $\mathrm{RbMgF}_{3}$.

The $\mathrm{Mn}^{2+}$ and $\mathrm{F} / \mathrm{Mn}$ complexes each exhibited a single PL emission in each compound, which occurred due to radiative transitions from the ${ }^{4} \mathrm{~T}_{1}\left({ }^{4} \mathrm{G}\right)$ states to the ${ }^{6} \mathrm{~A}_{1}\left({ }^{6} \mathrm{~S}\right)$ ground states for $\mathrm{Mn}^{2+}$, and the perturbed versions of these states for the F/Mn complexes. Exciting into higher energy states resulted in non-radiative transitions to the ${ }^{4} \mathrm{~T}_{1}\left({ }^{4} \mathrm{G}\right)$ state prior to the subsequent radiative transition. We then expected that by varying the emission wavelength $\left(\lambda_{\mathrm{em}}\right)$ used to detect the PL excitations we would observe similar behaviour for each of the F/Mn transitions, and conflicting behaviour for any excitation component unrelated to the complexes. This technique, labeled here as emission-wavelength-dependent excitation spectra fitting, is explored in this section.

For all $\alpha-\mathrm{F} / \mathrm{Mn}$ and $\beta-\mathrm{F} / \mathrm{Mn}$ complexes in each compound, excitation spectra were obtained for at least 4 distinct emission wavelengths that spanned the F/Mn emission band. The spectra were fitted to a sum of multiple Gaussians and the behaviour of each component was studied as a function of the emission wavelength. The components that exhibited the same emission-wavelength-dependence were attributed to F/Mn complexes. Additional components were attributed to alternative impurity and defect centres. All samples were irradiated with an X-ray dose of approximately 360 Gy prior to PL measurements.

\subsection{1 $\mathrm{NaMgF}_{3}: \mathrm{Mn}$}

Only a single $\mathrm{F} / \mathrm{Mn}$ complex, designated an $\alpha-\mathrm{F} / \mathrm{Mn}$ complex, was observed after irradiation in $\mathrm{NaMgF}_{3}: \mathrm{Mn}$. This was expected due to the equivalent $\mathrm{MgF}_{6}$ octahedra. The PL excitation spectra where $\lambda_{\mathrm{em}}=690 \mathrm{~nm}, \lambda_{\mathrm{em}}=710 \mathrm{~nm}, \lambda_{\mathrm{em}}=730 \mathrm{~nm}$, and $\lambda_{\mathrm{em}}=750 \mathrm{~nm}$, 


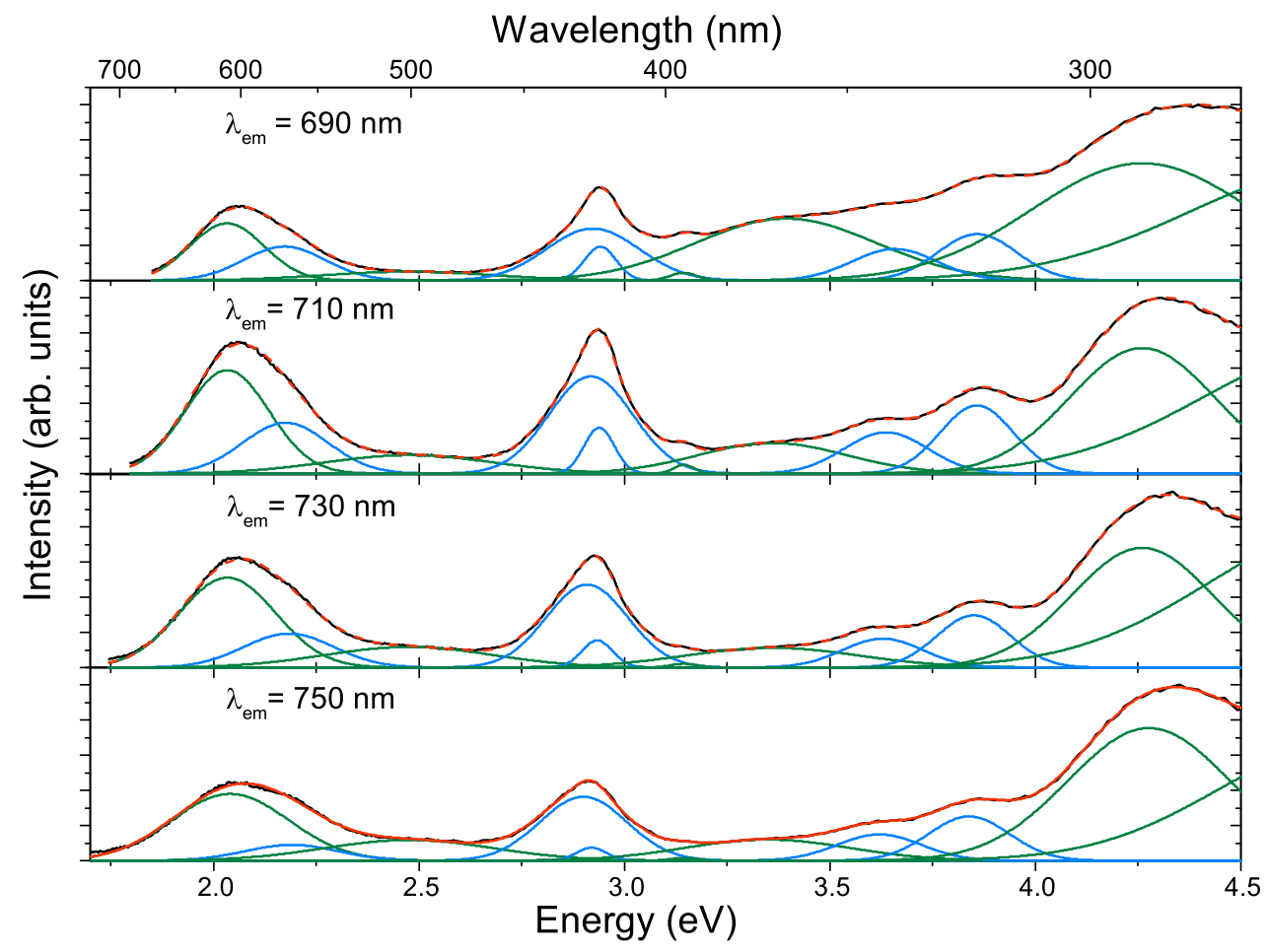

Figure 4.11: PL excitation spectra (black lines) for $\mathrm{NaMgF}_{3}: \mathrm{Mn}$ where $\lambda_{\mathrm{em}}=690 \mathrm{~nm}$, $\lambda_{\mathrm{em}}=710 \mathrm{~nm}, \lambda_{\mathrm{em}}=730 \mathrm{~nm}$, and $\lambda_{\mathrm{em}}=750 \mathrm{~nm}$. Other lines represent the Gaussian excitation components due to $\alpha-\mathrm{F} / \mathrm{Mn}$ complexes (blue) and alternative centres (green), and the cumulative fit (orange dashed). 
are shown in figure 4.11. Each spectrum was fitted to a sum of 11 Gaussians. The variations in the integrated areas and peak intensities of each component were monitored as a function of emission wavelength, where those arising from F/Mn complex transitions exhibited a similar dependence. The components peaking at $570 \mathrm{~nm}(2.18 \mathrm{eV}), 425$ $\mathrm{nm}(2.92 \mathrm{eV}), 422 \mathrm{~nm}(2.94 \mathrm{eV}), 341 \mathrm{~nm}(3.64 \mathrm{eV})$, and $321 \mathrm{~nm}(3.86 \mathrm{eV})$ were most intense at $\lambda_{\mathrm{em}}=710 \mathrm{~nm}$ and were attributed to F/Mn complexes. Additional components peaked at $610 \mathrm{~nm}(2.03 \mathrm{eV}), 501 \mathrm{~nm}(2.48 \mathrm{eV}), 394 \mathrm{~nm}(3.14 \mathrm{eV}), 369 \mathrm{~nm}(3.36 \mathrm{eV}), 291$ $\mathrm{nm}(4.26 \mathrm{eV})$, and $260 \mathrm{~nm}(4.76 \mathrm{eV})$, and all showed emission wavelength dependencies different to those of the F/Mn complexes. The additional components were attributed to $\mathrm{Mn}$-perturbed $\mathrm{F}_{3}$-centres, $\mathrm{F}_{3}$-centres, $\mathrm{Mn}^{2+}, \mathrm{O}^{2-}$-related centres, F-centres, and interstitial aggregates, respectively. These attributions were made based on comparisons with previously presented literature values [130] and are considered reliable in most cases. The attribution of the $610 \mathrm{~nm}$ component to an $\mathrm{Mn}$-perturbed $\mathrm{F}_{3}$-centre was made by considering a perturbation on the established $\mathrm{F}_{3}$-centre energy and should be considered tentative.

The F/Mn transitions were labeled using the term symbols that are accurate in labeling the $\mathrm{Mn}^{2+}$ states and should be considered as perturbed versions of those states. The data for $\mathrm{NaMgF}_{3}: \mathrm{Mn}$ are presented in table 4.2.

\subsection{2 $\mathrm{KMgF}_{3}: \mathrm{Mn}$}

Two distinct $\mathrm{F} / \mathrm{Mn}$ complexes were observed for $\mathrm{KMgF}_{3}: \mathrm{Mn}$. This was unexpected, as $\mathrm{KMgF}_{3}$ has the simple cubic perovskite structure and equivalent $\mathrm{MgF}_{6}$ octahedra. Additionally, only a single $\mathrm{Mn}^{2+}$ emission was seen in the PL. One complex produced a PL emission near $695 \mathrm{~nm}$, similar to that of $\mathrm{NaMgF}_{3}: \mathrm{Mn}$ and is thus designated an $\alpha-\mathrm{F} / \mathrm{Mn}$ complex. The second complex produced a PL emission near $610 \mathrm{~nm}$ that is designated a $\beta$-F/Mn complex. Emission-wavelength-dependent excitation spectra fitting was performed for each complex using different ranges of emission wavelengths.

The PL excitation spectra for the $\alpha-\mathrm{F} / \mathrm{Mn}$ complexes are shown in figure 4.12 , where $\lambda_{\mathrm{em}}=680 \mathrm{~nm}, \lambda_{\mathrm{em}}=700 \mathrm{~nm}, \lambda_{\mathrm{em}}=750 \mathrm{~nm}$, and $\lambda_{\mathrm{em}}=780 \mathrm{~nm}$. Each spectrum was fitted to a sum of 13 Gaussians and variations in the peak intensity and area of each component were monitored as a function of emission wavelength. The components peaking at 613 $\mathrm{nm}(2.02 \mathrm{eV}), 423 \mathrm{~nm}(2.93 \mathrm{eV}), 422 \mathrm{~nm}(2.94 \mathrm{eV}), 341 \mathrm{~nm}(3.63 \mathrm{eV})$, and $320 \mathrm{~nm}(3.88 \mathrm{eV})$ were most intense at $\lambda_{\mathrm{em}}=700 \mathrm{~nm}$ and were attributed to $\alpha-\mathrm{F} / \mathrm{Mn}$ complexes. Additional components peaked at $563 \mathrm{~nm}(2.20 \mathrm{eV}), 544 \mathrm{~nm}(2.28 \mathrm{eV}), 443 \mathrm{~nm}(2.80 \mathrm{eV}), 409 \mathrm{~nm}$ (3.03 eV), $396 \mathrm{~nm}(3.13 \mathrm{eV}), 374 \mathrm{~nm}(3.32 \mathrm{eV}), 293 \mathrm{~nm}(4.23 \mathrm{eV})$, and $274 \mathrm{~nm}(4.52 \mathrm{eV})$. 


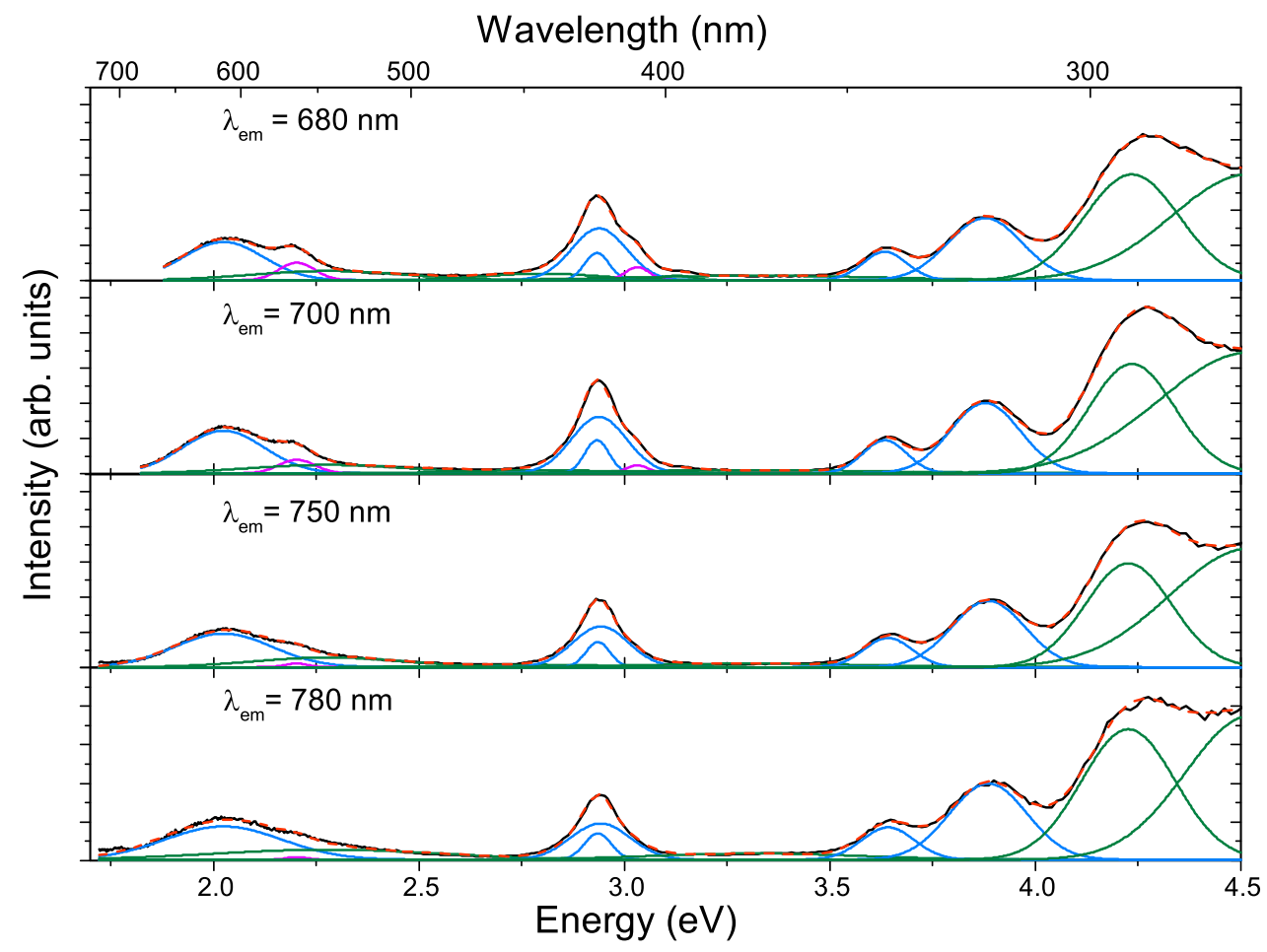

Figure 4.12: PL excitation spectra (black lines) for $\mathrm{KMgF}_{3}: \mathrm{Mn}$ where $\lambda_{\mathrm{em}}=680 \mathrm{~nm}, \lambda_{\mathrm{em}}=$ $700 \mathrm{~nm}, \lambda_{\mathrm{em}}=750 \mathrm{~nm}$, and $\lambda_{\mathrm{em}}=780 \mathrm{~nm}$. Other lines represent the Gaussian excitation components due to $\alpha-\mathrm{F} / \mathrm{Mn}$ complexes (blue), $\beta-\mathrm{F} / \mathrm{Mn}$ complexes (purple), alternative centres (green), and the cumulative fit (orange dashed). 
These were attributed to $\beta-\mathrm{F} / \mathrm{Mn}$ complexes, $\mathrm{K}$-vacancy-related centres, $\mathrm{F}_{2}$-centres, $\beta$ $\mathrm{F} / \mathrm{Mn}$ complexes, $\mathrm{Mn}^{2+}, \mathrm{O}^{2-}$-related centres, and F-centre variants, respectively. These attributions were based entirely on previously published data for the $\mathrm{KMgF}_{3}$ compound $[106,108,131]$. The data for $\mathrm{KMgF}_{3}: \mathrm{Mn}$ are summarised in table 4.2.

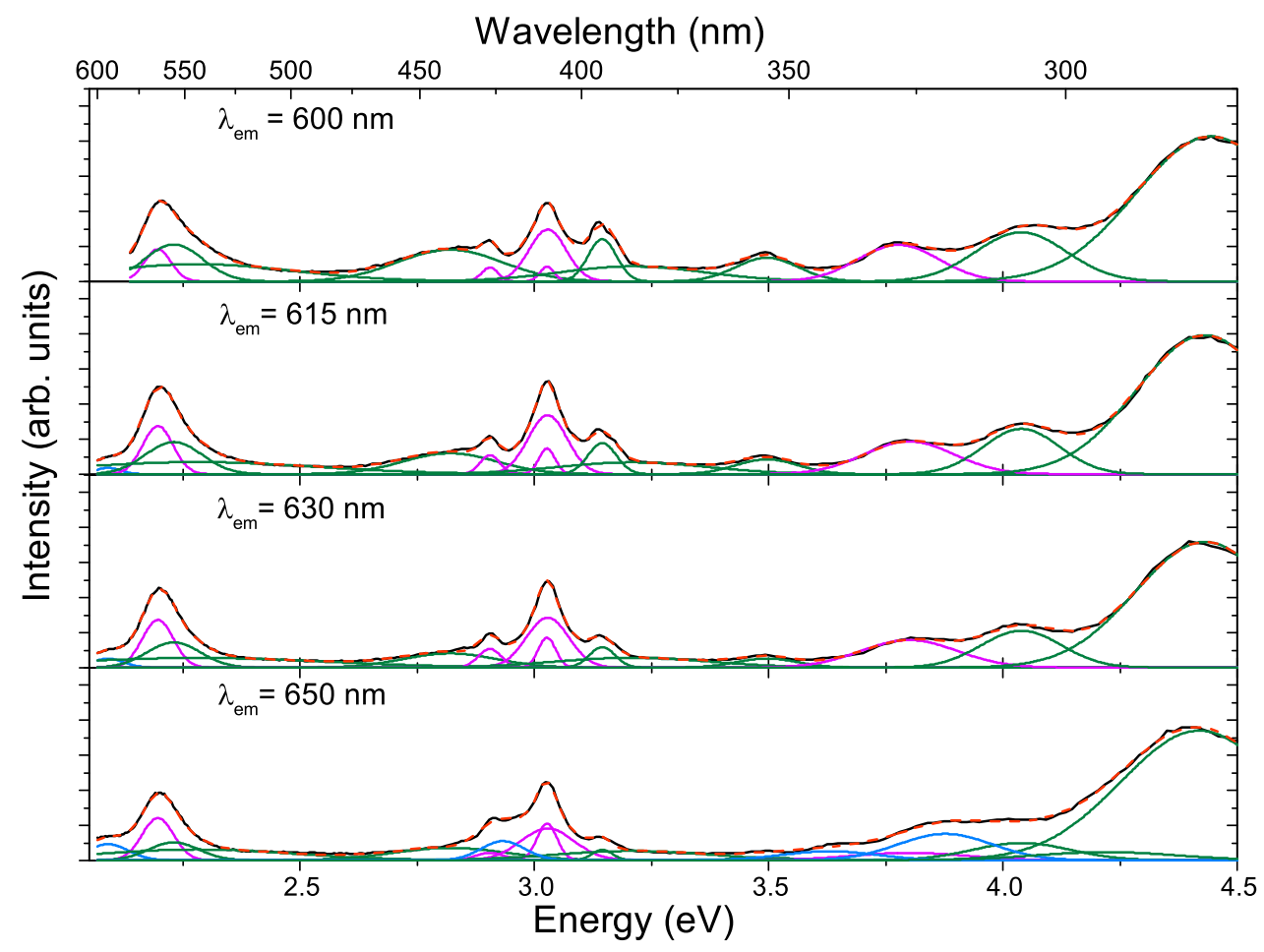

Figure 4.13: PL excitation spectra (black lines) for $\mathrm{KMgF}_{3}: \mathrm{Mn}$ where $\lambda_{\mathrm{em}}=600 \mathrm{~nm}, \lambda_{\mathrm{em}}=$ $615 \mathrm{~nm}, \lambda_{\mathrm{em}}=630 \mathrm{~nm}$, and $\lambda_{\mathrm{em}}=650 \mathrm{~nm}$. Other lines represent the Gaussian excitation components due to $\alpha-\mathrm{F} / \mathrm{Mn}$ complexes (blue), $\beta-\mathrm{F} / \mathrm{Mn}$ complexes (purple), alternative centres (green), and the cumulative fit (orange dashed).

The PL excitation spectra for the $\beta$-F/Mn complexes were investigated for $\lambda_{\mathrm{em}}=600$ $\mathrm{nm}, \lambda_{\mathrm{em}}=615 \mathrm{~nm}, \lambda_{\mathrm{em}}=630 \mathrm{~nm}$, and $\lambda_{\mathrm{em}}=650 \mathrm{~nm}$, and are shown in figure 4.13. Each spectrum was fitted to a sum of 14 Gaussians and each component was investigated as a function of emission wavelength. Those peaks occurring at $565 \mathrm{~nm}(2.20 \mathrm{eV}), 427 \mathrm{~nm}$ $(2.91 \mathrm{eV}), 410 \mathrm{~nm}(3.03 \mathrm{eV}), 326 \mathrm{~nm}(3.80 \mathrm{eV})$, and $307 \mathrm{~nm}(4.04 \mathrm{eV})$ had maximum intensities for $\lambda_{\mathrm{em}}=615 \mathrm{~nm}$ and were attributed to $\beta-\mathrm{F} / \mathrm{Mn}$ complexes. Additional peaks were fitted at $593 \mathrm{~nm}(2.09 \mathrm{eV}), 556 \mathrm{~nm}(2.23 \mathrm{eV}), 440 \mathrm{~nm}(2.82 \mathrm{eV}), 394 \mathrm{~nm}(3.14 \mathrm{eV}), 386 \mathrm{~nm}$ $(3.21 \mathrm{eV}), 355 \mathrm{~nm}(3.50 \mathrm{eV})$, and $280 \mathrm{~nm}(4.43 \mathrm{eV})$. These were attributed to $\alpha-\mathrm{F} / \mathrm{Mn}$ complexes, $\mathrm{Mn}^{2+}, \mathrm{F}_{2}$-centres, $\mathrm{Mn}^{2+}, \mathrm{O}^{2-}$-related centres, $\mathrm{Mn}^{2+}$, and F-centres, respectively. The data for the $\beta-\mathrm{F} / \mathrm{Mn}$ complexes are summarised in table 4.2. 


\subsection{3 $\mathrm{RbMgF}_{3}: \mathrm{Mn}$}

A single type of F/Mn complex fitting the $\alpha$-F/Mn complex description was observed in $\mathrm{RbMgF}_{3}: \mathrm{Mn}$ despite the existence of inequivalent $\mathrm{MgF}_{6}$ sites in the lattice. The PL excitation spectra for the complexes are shown in figure 4.14 , where $\lambda_{\mathrm{em}}=660 \mathrm{~nm}, \lambda_{\mathrm{em}}$

$=690 \mathrm{~nm}, \lambda_{\mathrm{em}}=720 \mathrm{~nm}$, and $\lambda_{\mathrm{em}}=750 \mathrm{~nm}$. Each spectrum was fitted to the sum of 13 Gaussians and each component was monitored as a function of emission wavelength. Those components peaking at $575 \mathrm{~nm}(2.16 \mathrm{eV}), 423 \mathrm{~nm}(2.93 \mathrm{eV}), 342 \mathrm{~nm}(3.63 \mathrm{eV})$, and $321 \mathrm{~nm}(3.86 \mathrm{eV})$ had maximum intensities at $\lambda_{\mathrm{em}}=660 \mathrm{~nm}$ and were attributed to the $\alpha$-F/Mn complexes. Additional components were fitted at $614 \mathrm{~nm}(2.02 \mathrm{eV}), 534 \mathrm{~nm}(2.32$ $\mathrm{eV}), 434 \mathrm{~nm}(2.86 \mathrm{eV}), 396 \mathrm{~nm}(3.13 \mathrm{eV}), 380 \mathrm{~nm}(3.26 \mathrm{eV}), 358 \mathrm{~nm}(3.46 \mathrm{eV}), 332 \mathrm{~nm}(3.74$ $\mathrm{eV}), 295 \mathrm{~nm}(4.21 \mathrm{eV})$, and $264 \mathrm{~nm}(4.69 \mathrm{eV})$. These were attributed to an unknown defect/Mn complex, $\mathrm{Mn}^{2+}, \mathrm{Mn}^{2+}, \mathrm{Mn}^{2+}, \mathrm{O}^{2-}$-related centres, $\mathrm{Mn}^{2+}, \mathrm{Mn}^{2+}$, F-centres, and interstitials, respectively. These attributions were made based on comparisons with previously published data for $\mathrm{RbMgF}_{3}[57,108]$. The $\mathrm{RbMgF}_{3}: \mathrm{Mn} \alpha-\mathrm{F} / \mathrm{Mn}$ complex data are given in table 4.2 .

\subsubsection{Comparison and Summary}

The F/Mn complex transition energies were determined for $\mathrm{NaMgF}_{3}: \mathrm{Mn}, \mathrm{KMgF}_{3}: \mathrm{Mn}$, and $\mathrm{RbMgF}_{3}: \mathrm{Mn}$ using an emission-wavelength-dependent excitation spectra fitting technique. The transition energies relative to the ground state for each compound are shown in table 4.2. It should be reiterated that the term symbols used to express the electronic states do not adequately represent the electronic states of the F/Mn complexes and are included to indicate the $\mathrm{Mn}^{2+}$ state from which the transitions were perturbed by coupling with an F-centre. All F/Mn transitions were largely analogous to the unperturbed $\mathrm{Mn}^{2+}$ transitions in terms of line structure and relative intensities, indicating that this labeling scheme should be useful in the analysis of such complexes.

For all samples, a relatively high X-ray dose (360 Gy) produced well-defined F/Mn complexes with high PL intensities. The relative intensities of the $\mathrm{Mn}^{2+} \mathrm{PL}$ and the F/Mn PL may be used to estimate the relative oscillator strengths of the two luminescence centres. The F/Mn PL intensities were approximately $1 \%-10 \%$ as intense as those of the $\mathrm{Mn}^{2+}$ sites in all cases. However, the $\mathrm{Mn}^{2+}$ concentrations (1\%) were large compared to the expected populations of F-centres $(<0.01 \%)$. Furthermore, only a fraction of those created F-centres could form F/Mn complexes. We conclude that the oscillator strengths of the F/Mn complexes are greater than those of the $\mathrm{Mn}^{2+}$ in all samples studied here 


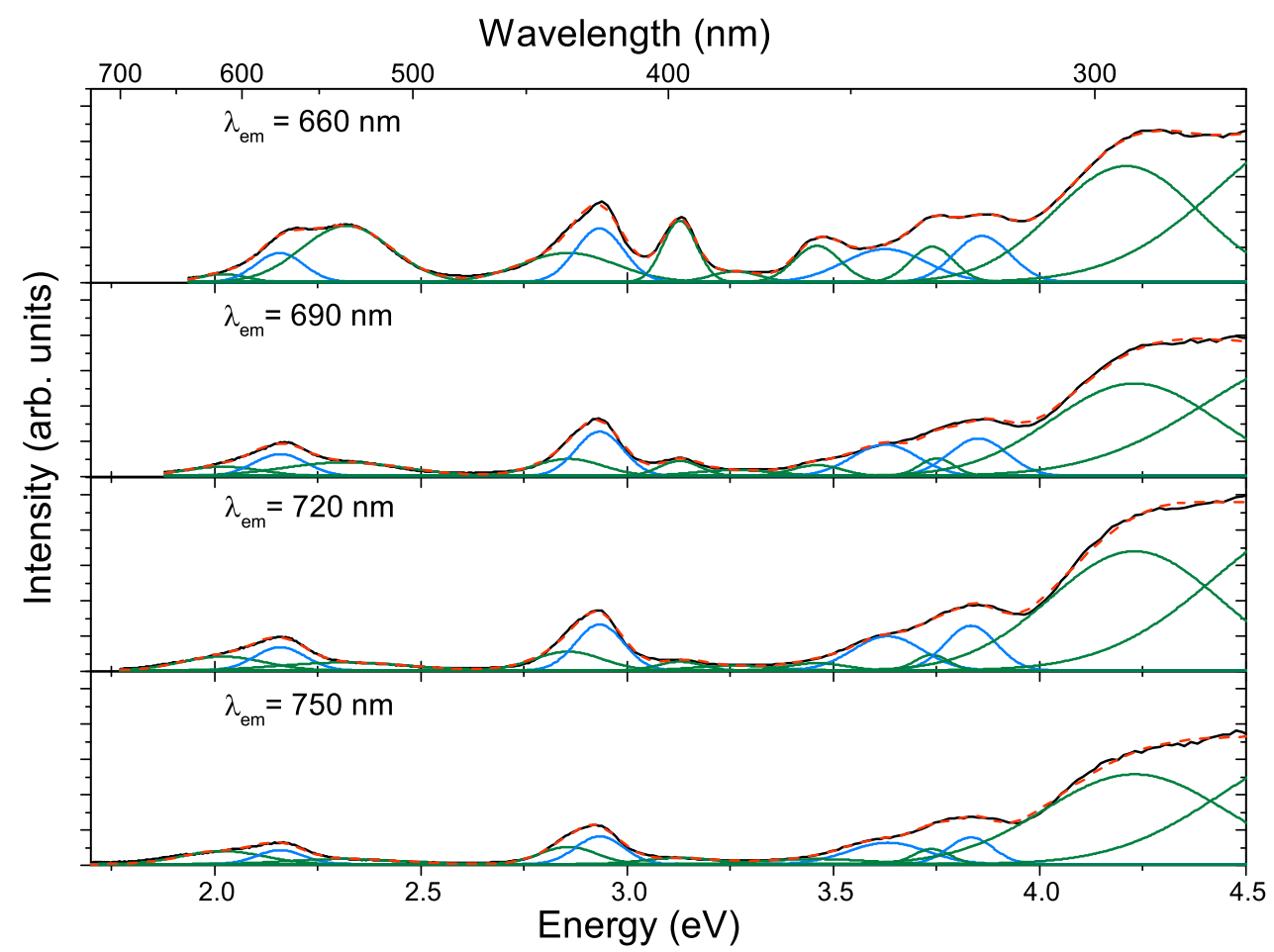

Figure 4.14: PL excitation spectra (black lines) for $\mathrm{RbMgF}_{3}: \mathrm{Mn}$ where $\lambda_{\mathrm{em}}=660 \mathrm{~nm}$, $\lambda_{\mathrm{em}}=690 \mathrm{~nm}, \lambda_{\mathrm{em}}=720 \mathrm{~nm}$, and $\lambda_{\mathrm{em}}=750 \mathrm{~nm}$. Other lines represent the Gaussian excitation components due to $\alpha-\mathrm{F} / \mathrm{Mn}$ complexes (blue), alternative centres (green), and the cumulative fit (orange dashed).

and this feature will be explored in the following section (4.3). An increased oscillator strength was expected based on the spin-coupling interpretation of the complexes (figure 4.10).

The transition energies of the F/Mn complexes were perturbed from those of $\mathrm{Mn}^{2+}$ in each host compound as expected. However, the degree of perturbation depended on the particular transition in all cases, which would not be expected based solely on a spincoupling mechanism as all excited states of the unperturbed $\mathrm{Mn}^{2+}$ ion have the same spin $(S=3 / 2)$. The crystal field perturbed ${ }^{4} G$ energy levels relative to the ${ }^{6} S$ ground state were always reduced in energy, leading to PL emissions at longer wavelengths than that of $\mathrm{Mn}^{2+}$ for all samples. Conversely, the ${ }^{4} \mathrm{D}$ energy levels were increased in energy relative to the ground state in all samples, leading to higher energy excitations than those of $\mathrm{Mn}^{2+}$. As both unperturbed levels have the same spin this behaviour indicates that the electronic coupling mechanism of the F/Mn structure is more complicated than a simple spin addition/subtraction. We have found that the perturbations on the levels of the $\mathrm{Mn}^{2+}$ ion are additionally dependent on the total orbital angular momentum quantum 
number $L$.

No obvious trends in the F/Mn complex energies were observed for increasing $A$ site ionic radii through the series of studied fluoroperovskites. This behaviour contrasts that of the $\mathrm{Mn}^{2+}$ ion, where the energies were clearly dependent on the crystal field produced by the host compound. The $\alpha-\mathrm{F} / \mathrm{Mn}$ complexes that were observed in all compounds had similar transition energies. The observation of two distinct complexes in $\mathrm{KMgF}_{3}: \mathrm{Mn}$, where the $\beta-\mathrm{F} / \mathrm{Mn}$ complex was perturbed from the $\mathrm{Mn}^{2+}$ ion to a significantly lesser degree than the $\alpha-\mathrm{F} / \mathrm{Mn}$ complex, was unexpected. However, similar structures have been previously observed in the $\mathrm{KMgF}_{3}: \mathrm{Mn}$ host, particularly after low-temperature irradiations [125]. It has been suggested that the F/Mn complexes may themselves be perturbed by nearby point defects $[125,126]$. The $\beta-\mathrm{F} / \mathrm{Mn}$ complex may result from an $\alpha-\mathrm{F} / \mathrm{Mn}$ complex that is perturbed by an interstitial fluorine adjacent to the $\mathrm{MnF}_{6}$ octahedron [125]. The observation of this structure after room temperature irradiation remains unexpected and may be indicative of impurity sites that served to stabilise interstitials, i.e. the presence of $\mathrm{H}_{\mathrm{A}}$-centres. However, the lack of any $\beta$-F/Mn complexes in the other hosts $\left(\mathrm{NaMgF}_{3}: \mathrm{Mn}\right.$ and $\left.\mathrm{RbMgF}_{3}: \mathrm{Mn}\right)$ marks this interpretation as tentative.

Finally, a perturbed ${ }^{4} \mathrm{~T}_{1}\left({ }^{4} \mathrm{G}\right)$ level did not appear in any spectrum, suggesting that the level may remain spin-forbidden despite the electron coupling. This indicates that not only are spin and orbital angular momenta important aspects of the complex, but that symmetry may also have a strong affect on the oscillator strengths. Substitution of a trapped electron for a $\mathrm{F}^{-}$ligand should not overwhelmingly affect the crystal field, as the negative charge on the substituted ligand persists and the $O_{h}$ crystal field remains. However, some distortion of the lattice is likely to occur about the $\mathrm{F}^{-}$vacancy, which may affect the symmetry selection rules for the F/Mn transitions.

The section has presented data that were used to establish the specific transition energies for all $\mathrm{F} / \mathrm{Mn}$ complexes observed in the $\mathrm{AMgF}_{3}$ fluoroperovskites after room temperature irradiation. Given the potential use cases of defect-impurity complexes, particularly in radiation dose monitoring via RPL, the data should assist in future experimental and theoretical investigations. Similarities are expected between the fluoroperovskites and other fluoride host compounds doped with $\mathrm{Mn}^{2+}$, as with $\mathrm{MgF}_{2}: \mathrm{Mn}$ and $\mathrm{CaF}_{2}: \mathrm{Mn}$, compounding the merits of the data. 


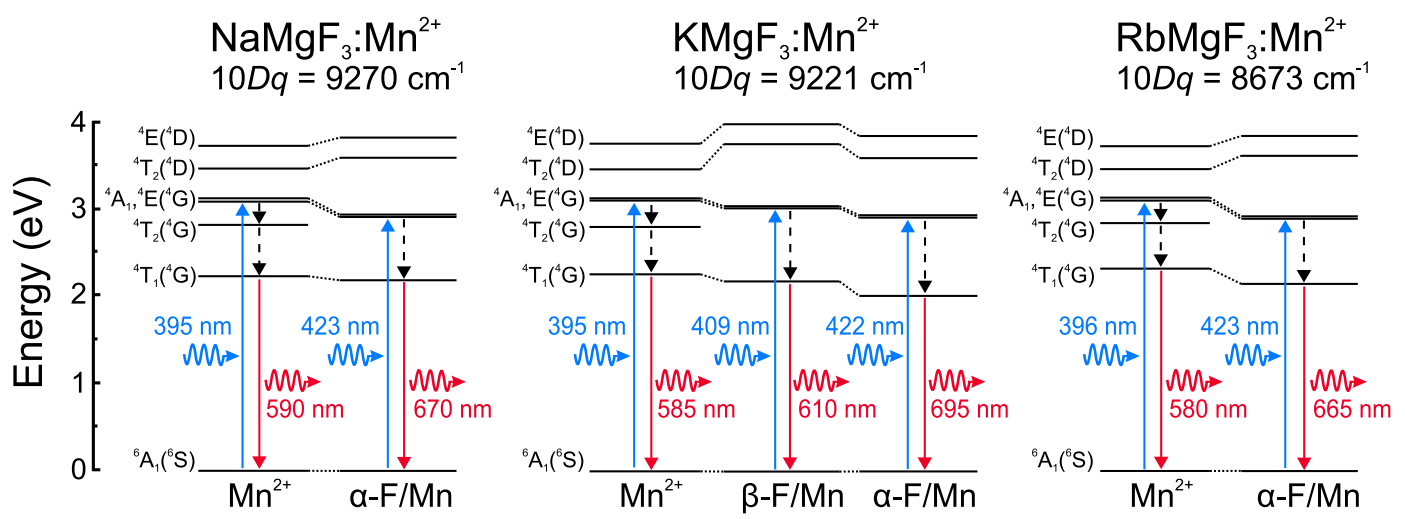

Figure 4.15: Energy diagrams of the $\mathrm{Mn}^{2+}$ and spin-allowed $\mathrm{F} / \mathrm{Mn}$ complex transitions in $\mathrm{NaMgF}_{3}: \mathrm{Mn}, \mathrm{KMgF}_{3}: \mathrm{Mn}$, and $\mathrm{RbMgF}_{3}: \mathrm{Mn}$. Solid arrows are optical transitions and dashed arrows are non-radiative transitions.

\begin{tabular}{ccccc}
\hline Perturbed Transition & $\mathrm{NaMgF}_{3}: \mathrm{Mn}$ & $\mathrm{KMgF}_{3}: \mathrm{Mn}$ & $\mathrm{KMgF}_{3}: \mathrm{Mn}$ & $\mathrm{RbMgF}_{3}: \mathrm{Mn}$ \\
\hline \hline $\mathrm{F} / \mathrm{Mn}$ emission $(\mathrm{eV})$ & 1.85 & 1.78 & 2.03 & 1.87 \\
\hline${ }^{4} \mathrm{~T}_{1}(\mathrm{G})(\mathrm{eV})$ & 2.19 & 2.02 & 2.20 & 2.16 \\
\hline${ }^{4} \mathrm{~A}_{1}(\mathrm{G})(\mathrm{eV})$ & 2.92 & 2.93 & 3.03 & 2.91 \\
\hline${ }^{4} \mathrm{E}(\mathrm{G})(\mathrm{eV})$ & 2.93 & 2.94 & 3.03 & 2.93 \\
\hline${ }^{4} \mathrm{~T}_{2}(\mathrm{D})(\mathrm{eV})$ & 3.62 & 3.63 & 3.80 & 3.63 \\
\hline${ }^{4} \mathrm{E}(\mathrm{D})(\mathrm{eV})$ & 3.84 & 3.88 & 4.04 & 3.86 \\
\hline
\end{tabular}

Table 4.2: Summary of the F-centre/Mn transition energies for $\mathrm{NaMgF}_{3}: \mathrm{Mn}, \mathrm{KMgF}_{3}: \mathrm{Mn}$ and $\mathrm{RbMgF}_{3}: \mathrm{Mn}$, relative to the ground state. 


\subsection{Dosimetric Properties of $\mathrm{NaMgF}_{3}: \mathrm{Mn}$}

The PL characterisations of the fluoroperovskites revealed several key features that may find real-world applications in radiation dosimetry. OSL excitations were observed for all samples that may be used for total dose monitoring with destructive readout. F/Mn complexes were also seen that may be used for non-destructive RPL dosimetry in cooperation with the OSL. $\mathrm{NaMgF}_{3}$ is an ideal host compound for dosimetry applications due to its near tissue-equivalence. As such, an extensive investigation into the luminescence properties of $\mathrm{NaMgF}_{3}: \mathrm{Mn}$, where the Mn concentration was varied from $0.1 \%$ to $5 \%$, is presented in this section. Samples were characterised via optical absorption, PL, RPL, OSL, and RL. Ultimately, the viability of the compound as a dosimeter material was established.

\subsubsection{Optical Absorption}

The synthesised samples of $\mathrm{NaMgF}_{3}: \mathrm{Mn}$, where $\mathrm{Mn}=0.1 \%, 0.5 \%$, and $1 \%$, were sufficiently transparent such that transmittance measurements were possible. When $\mathrm{Mn}$ $=5 \%$ transmittance measurements were not possible due to the sample opacity. The measurements prior to irradiation showed no significant structure other than a broad scattering background. $\mathrm{Mn}^{2+}$ absorptions were not observed due to the low oscillator strengths of the $3 d^{5}$ transitions. After exposure to X-ray irradiation, with doses of 180 Gy, clear absorptions were induced at short wavelengths extending into the UV. The data obtained prior to irradiation were subtracted from those obtained after irradiation in order to produce only the radiation-induced changes in the transmittance and these data were expressed in terms of absorption coefficients using equation 3.1. The radiationinduced absorption coefficients are plotted in figure 4.16 (a). All samples showed similar absorptions after irradiation, with clear peaks at approximately $340 \mathrm{~nm}(3.65 \mathrm{eV})$ and additional absorption components extending to higher energies.

All absorption spectra could be fitted to the sum of 3 Gaussians, where the peak energies of the components were the same for each spectrum. The individual components peaked at $342 \mathrm{~nm}(3.63 \mathrm{eV}), 292 \mathrm{~nm}(4.25 \mathrm{eV})$, and $255 \mathrm{~nm}(4.86 \mathrm{eV})$. The integrated intensities of each component as a function of Mn concentration are shown in figure 4.16 (b) where it is clear that the peak absorption coefficients, and therefore defect concentrations, decreased for increasing $\mathrm{Mn}^{2+}$ concentrations. For the X-ray energies used in this work, increasing the concentration of $\mathrm{Mn}^{2+}$ in the lattice would slightly increase the total dose absorbed by the sample, suggesting a slightly greater concentration of 
radiation-induced defects. The most likely explanation for the decreased concentrations of defects is that $\mathrm{Mn}^{2+}$ acted as a recombination centre for the free electrons and holes produced during irradiation. The probability of recombination via $\mathrm{Mn}^{2+}$ sites increased with Mn concentration and consequently reduced the likelihood of the free charges being trapped by defects. It is also possible that the incorporation of $\mathrm{Mn}^{2+}$ in the lattice decreased the total concentration of defects by reducing the number of available lattice vacancies, though this is considered unlikely as the increased dopant concentrations would produce a greater number of lattice distortions.
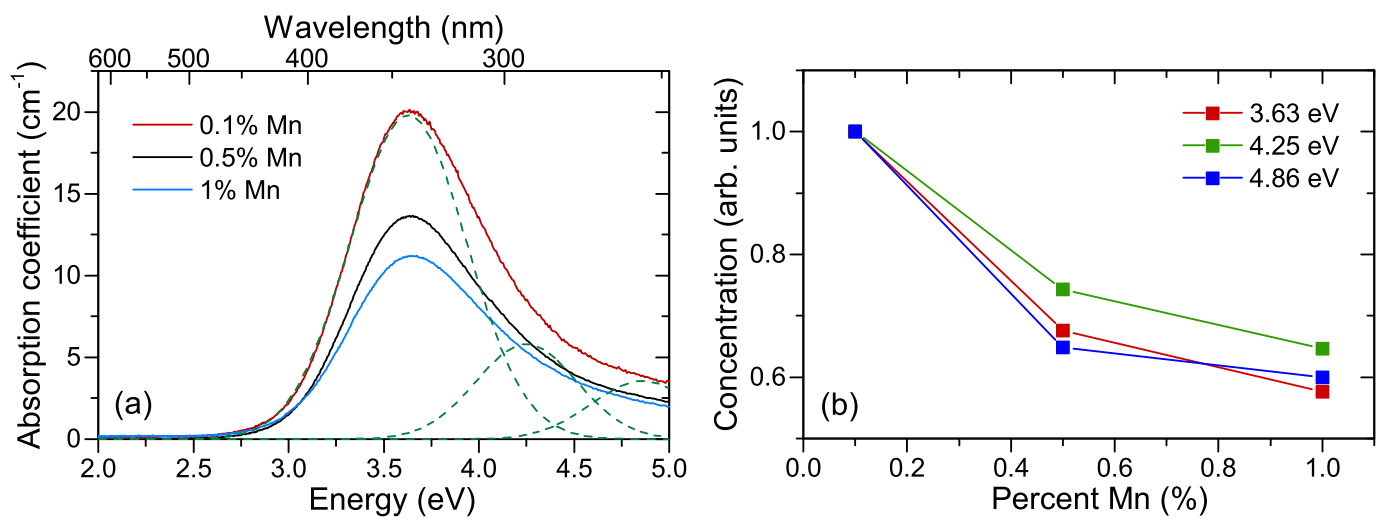

Figure 4.16: (a) Radiation-induced absorption coefficients of $\mathrm{NaMgF}_{3}: \mathrm{Mn}$ after an X-ray dose of 180 Gy for $\mathrm{Mn}=0.1 \%$ (red), $0.5 \%$ (black), and $1 \%$ (red). Green dashed lines are the fitted Gaussian components for the $0.1 \% \mathrm{Mn}$. (b) Normalised defect concentrations as a function of $\mathrm{Mn}$ concentration for the $\mathrm{H}_{\mathrm{A}}$-centres (red), F-centres (green), and interstitials (blue).

The high energy peaks at $292 \mathrm{~nm}$ and $255 \mathrm{~nm}$ have been previously observed in nominally pure samples of $\mathrm{NaMgF}_{3}$ and were established to arise from the formation of F-centres and interstitial deep traps, respectively [130,132]. The main absorption peak at $342 \mathrm{~nm}$ has not been observed at room temperature in the compound, though a similar peak at $350 \mathrm{~nm}$ was reported at low-temperatures for low-temperature-irradiated pure $\mathrm{NaMgF}_{3}$ [130]. This peak was attributed to hole traps that could be either self-trapped holes or a type of $\mathrm{H}$-centre. Self-trapped holes typically present as $\mathrm{F}_{2}^{-}$molecules in fluoride systems, where a hole is shared by neighbouring $\mathrm{F}^{-}$ions [130]. The H-centre is generally described by an interstitial $\mathrm{F}^{-}$ion that traps a hole, forming a $\mathrm{F}^{0}$ interstitial atom. The absence of a $342 \mathrm{~nm}$ absorption in pure samples studied at room temperature suggests that the $\mathrm{Mn}^{2+}$ ions served to stabilise the hole trap at higher temperatures. We tentatively ascribe the defect to an $\mathrm{H}_{\mathrm{A}}$-centre, i.e. an $\mathrm{H}_{\mathrm{Mn}}$-centre, where the $\mathrm{H}$ centre was stabilised by a nearby $\mathrm{Mn}^{2+}$ ion [42]. The presence of these interstitials could 
significantly affect the site symmetries of those nearby $\mathrm{Mn}^{2+}$ ions, potentially producing significantly distorted $\mathrm{Mn}^{2+}$ sites and the unexpected $\mathrm{Mn}^{2+}$ emission for the $\mathrm{NaMgF}_{3}: \mathrm{Mn}$ system.

\subsubsection{Photoluminescence}

The PL emission spectra for the different $\mathrm{Mn}^{2+}$ concentrations, where $\lambda_{\mathrm{ex}}=397 \mathrm{~nm}$, are shown in figure 4.17 (a). The data were normalised to the peak emission of the distorted site for all samples, whereupon it was clear that the non-distorted emission intensities increased relative to the distorted emissions for increased $\mathrm{Mn}^{2+}$ concentrations. The $\mathrm{O}^{2-}-$ related emission also decreased relative to the $\mathrm{Mn}^{2+}$ concentrations, particularly when increased from $0.1 \% \mathrm{Mn}$ to $0.5 \% \mathrm{Mn}$, suggesting that the degree of $\mathrm{O}^{2-}$ incorporation was independent of the Mn dopant concentration. The data suggest that there were fewer available distorted sites than non-distorted sites, such that when the $\mathrm{Mn}^{2+}$ concentration was increased, the ratio of the PL of non-distorted to distorted sites increased.

The spectra were fitted to Gaussians representing the two unique $\mathrm{Mn}^{2+}$ sites and used to determine the integrated intensities of each emission as a function of $\mathrm{Mn}$ concentration. The ratio of the two emissions, $R_{\mathrm{Mn}, \mathrm{PL}}$, is plotted in figure $4.17(\mathrm{~b}) . R_{\mathrm{Mn}, \mathrm{PL}}$ increased linearly for concentrations $\geq 0.5 \%$ and deviated from linearity for $0.1 \% \mathrm{Mn}$.

In order to better evaluate the actual concentrations of each site, PL lifetime measurements were performed for each of the $\mathrm{Mn}^{2+}$ sites. The excitation wavelength was $397 \mathrm{~nm}$ in both cases and the PL decays are shown in figure 4.17 (c). Both decays could be fitted to single exponential decays and were determined to be $\tau_{\mathrm{D}}=12.4 \mathrm{~ms}$ and $\tau_{\mathrm{N}}=80.2 \mathrm{~ms}$ for the distorted and non-distorted $\mathrm{Mn}^{2+}$ sites, respectively. PL lifetimes on the order of $10 \mathrm{~ms}$ are expected for low-oscillator strength transitions, such as the spin- and parityforbidden $\mathrm{Mn}^{2+} 3 d \rightarrow 3 d$ transitions [122]. The decreased lifetime for the distorted sites is indicative of a partial lifting of the forbidden aspects of the transitions, as expected for distorted sites [27].

The PL emission intensity, $I_{\mathrm{PL}}$, for a purely radiative transition is approximately proportional to the concentration of PL-active sites, $N_{\mathrm{PL}}$, and the inverse of the PL lifetime, $\tau$, such that $I_{\mathrm{PL}} \propto N_{\mathrm{PL}} / \tau$ [55]. We may use this property to estimate the actual concentrations of PL-active distorted and non-distorted $\mathrm{Mn}^{2+}$ sites. By assuming that the non-radiative decay from the higher excited states of $\mathrm{Mn}^{2+}$ to the ${ }^{4} \mathrm{~T}_{1}(\mathrm{G})$ level was rapid, the PL ratio $R_{\mathrm{Mn}, \mathrm{PL}}$ may be written

$$
R_{\mathrm{Mn}, \mathrm{PL}}=N_{\mathrm{N}} \tau_{\mathrm{D}} / N_{\mathrm{D}} \tau_{\mathrm{N}}
$$



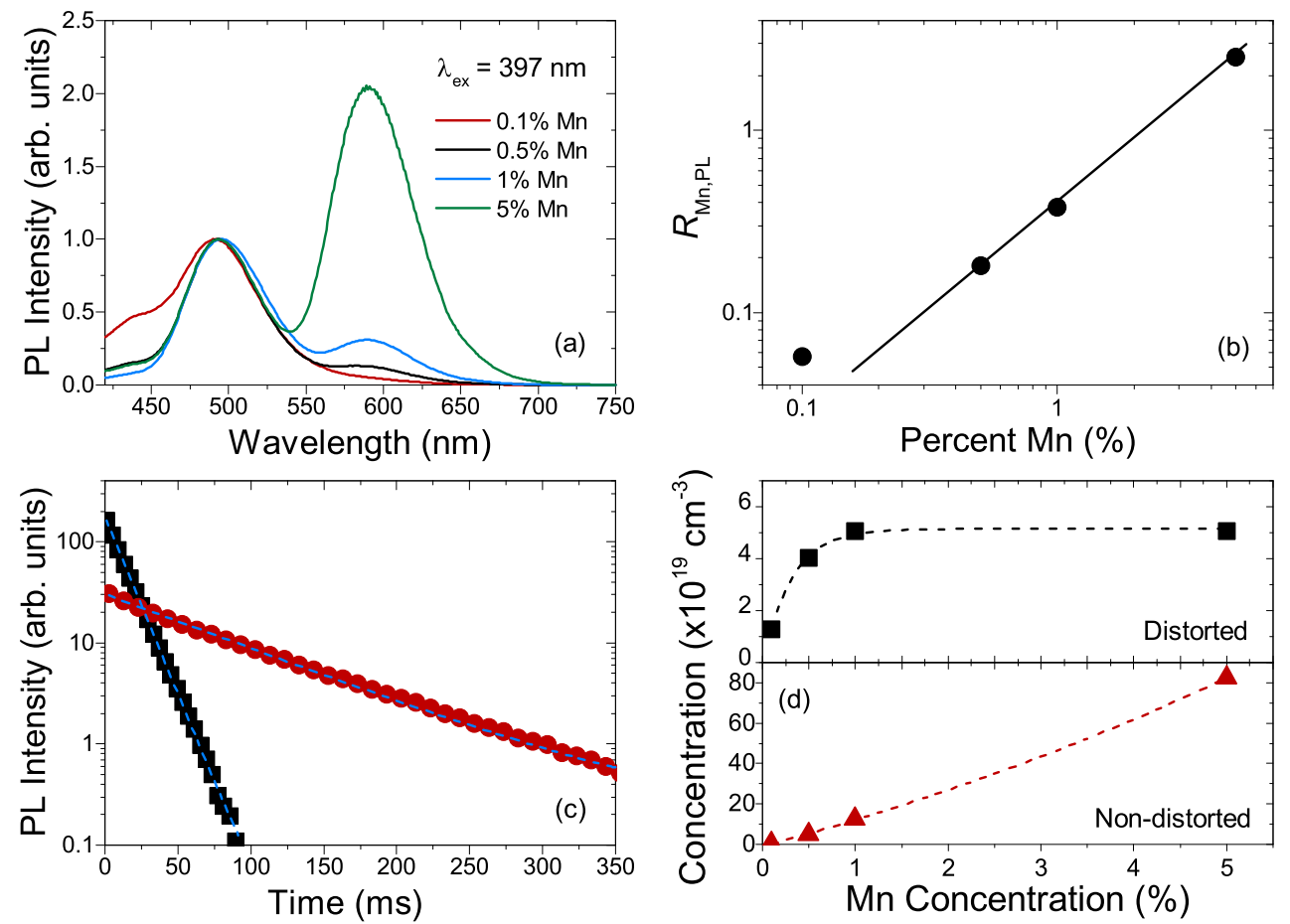

Figure 4.17: (a) PL emission spectra where $\lambda_{\mathrm{ex}}=397 \mathrm{~nm}$ for samples with nominal $\mathrm{Mn}$ concentrations of $0.1 \%$ (red), $0.5 \%$ (black), $1 \%$ (blue) and $5 \%$ (green). All spectra were normalised to the $495 \mathrm{~nm}$ peak. (b) Ratio of the integrated PL intensities of the nondistorted and distorted $\mathrm{Mn}^{2+}$ sites, $R_{\mathrm{Mn}, \mathrm{PL}}$, versus the $\mathrm{Mn}$ concentration. The solid line is a guide to the eye. (c) PL lifetime measurements for the distorted (black) and nondistorted (red) Mn sites for $0.5 \% \mathrm{Mn}$. Blue dashed lines are exponential fits to the data. (d) $\mathrm{Mn}^{2+}$ concentrations occupying distorted (black) and non-distorted (red) lattice positions, as calculated using equations 4.3 , versus the Mn concentration. 
where $N_{\mathrm{N}}$ and $N_{\mathrm{D}}$ are the concentrations of the non-distorted and distorted $\mathrm{Mn}^{2+}$ sites, respectively. The total $\mathrm{Mn}$ concentration, $N_{\mathrm{T}}$, can be calculated from the initial dopant fraction, $x$, via the relation

$$
N_{\mathrm{T}}=x / V_{\mathrm{f}}
$$

where $V_{\mathrm{f}}$ is the formula unit volume of $\mathrm{NaMgF}_{3}$ and $N_{\mathrm{T}}=N_{\mathrm{N}}+N_{\mathrm{D}}$. Equations 4.1 and 4.2 may be combined to express the site concentrations as

$$
\begin{gathered}
N_{\mathrm{N}}=N_{\mathrm{T}} \times R_{\mathrm{Mn}, \mathrm{PL}} \times \tau_{\mathrm{N}} /\left[R_{\mathrm{Mn}, \mathrm{PL}} \times \tau_{\mathrm{N}}+\tau_{\mathrm{N}}+\tau_{\mathrm{D}}\right], \\
N_{\mathrm{D}}=N_{\mathrm{T}} \times \tau_{\mathrm{D}} /\left[R_{\mathrm{Mn}, \mathrm{PL}} \times \tau_{\mathrm{N}}+\tau_{\mathrm{D}}\right] .
\end{gathered}
$$

The calculated concentrations $N_{\mathrm{N}}$ and $N_{\mathrm{D}}$ are plotted in figure 4.17 (d). This analysis reinforced the hypothesis that there existed a greater concentration of non-distorted sites and demonstrated that the concentration of distorted sites was fundamentally limited i.e. the number of available distorted sites did not scale with the total $\mathrm{Mn}^{2+}$ concentration. All distorted sites were occupied for $\geq 1 \%$ Mn concentrations. Once this saturation was achieved all additional $\mathrm{Mn}^{2+}$ occupied non-distorted sites and thus $R_{\mathrm{Mn} \text {,PL }}$ tended toward linearity for increasing Mn concentrations. The data also indicated that $\mathrm{Mn}^{2+}$ preferentially doped into distorted sites provided they were available, where for $0.1 \% \mathrm{Mn}$ the concentration of distorted sites was greater than that of the non-distorted sites.

\subsubsection{Radiophotoluminescence}

The F/Mn complexes in $\mathrm{NaMgF}_{3}: \mathrm{Mn}$ were discussed in detail in Section 4.2.1. The complexes were PL active and produced by X-ray irradiation and thus constituted RPL useful for radiation dose monitoring. It was determined that the F/Mn complex excitations extended out to approximately $570 \mathrm{~nm}$ and an additional excitation occurred in the region at approximately $605 \mathrm{~nm}$ that was attributed to $\mathrm{F}_{3, \mathrm{Mn}}$ centres. It was of interest to observe the behaviour of these complexes as a function of cumulative X-ray dose and this was completed for the $\mathrm{NaMgF}_{3}: 0.5 \% \mathrm{Mn}$ sample. The $0.5 \% \mathrm{Mn}$ concentration was selected due to the minor influence of the overlapping non-distorted $\mathrm{Mn}^{2+}$ emissions and the reduced effect on the tissue equivalence in comparison to higher dopant concentrations.

The low-energy RPL excitation bands of the F-centre + Mn centres are shown in fig- 
ure 4.18 (a) for cumulative X-ray doses ranging from 18 Gy to $5200 \mathrm{~Gy}$, where the PL excitation prior to irradiation was subtracted. It is clear that the excitation intensities increased with total radiation dose. This was expected as larger radiation doses should produce larger quantities of F-type centres that may then statistically form F/Mn complexes, up until the F-centre concentration saturates. Similar behaviour was observed for the RPL emissions when $\lambda_{\text {ex }}=570 \mathrm{~nm}$ after subtracting the spectrum prior to irradiation (figure 4.18 (b)). The inset to figure 4.18 (b) illustrates the variation in the RPL emission intensity over time during continuous stimulation into the excitation band at $\lambda_{\text {ex }}=566 \mathrm{~nm}$. The RPL was stable for at least 30 minutes, demonstrating that the RPL can be non-destructively probed by exploiting the existence of low-energy F/Mn excitation bands. The energy of the $566 \mathrm{~nm}$ stimulation was insufficient in releasing any trapped charges.
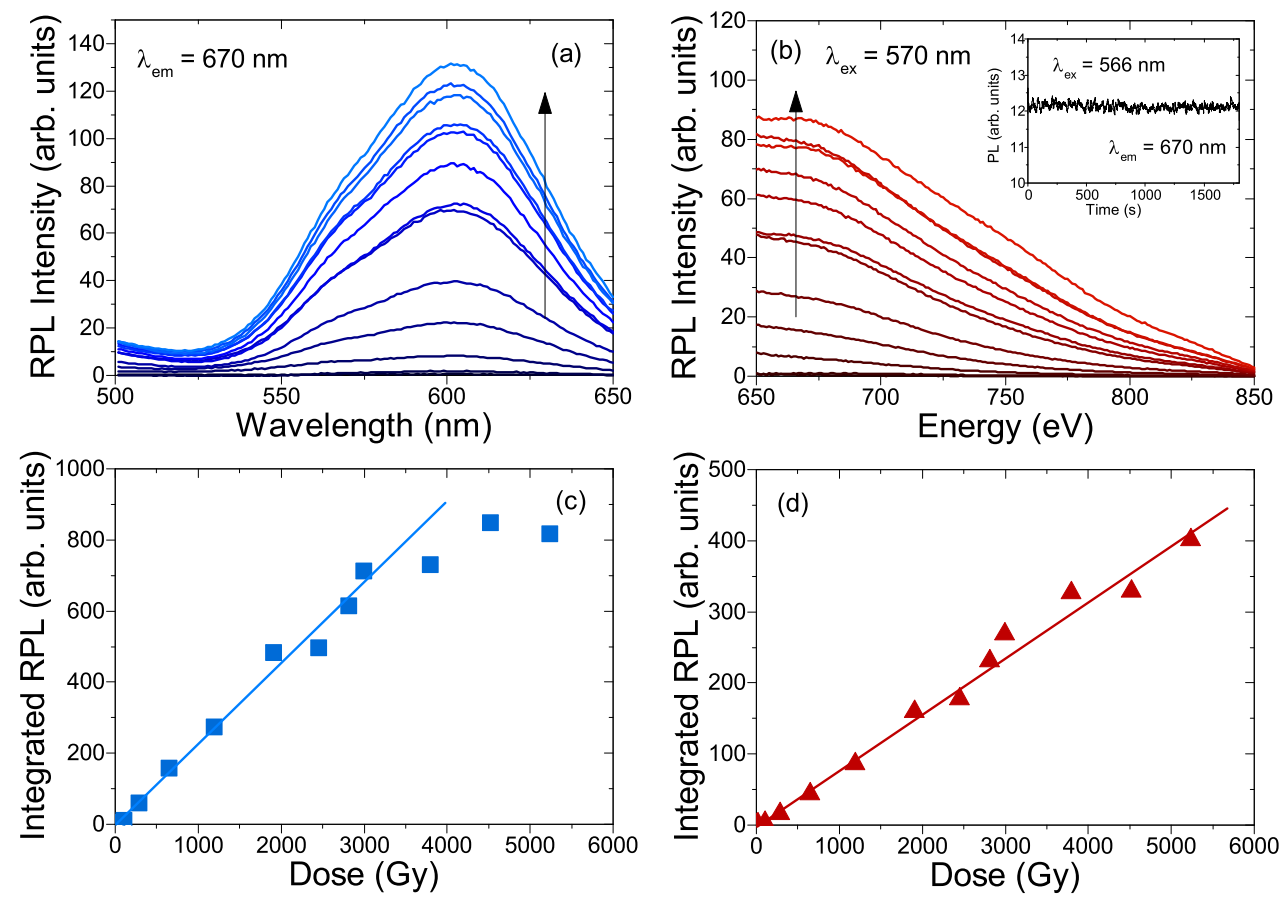

Figure 4.18: (a) Low-energy region of the PL excitation for $0.5 \% \mathrm{Mn}$ where $\lambda_{\mathrm{em}}=670$ $\mathrm{nm}$ for cumulative X-ray doses ranging from $18 \mathrm{~Gy}$ to $5200 \mathrm{~Gy}$. The arrow indicates the trend for increased X-ray doses. (b) PL emission spectra for $0.5 \% \mathrm{Mn}$ where $\lambda_{\mathrm{ex}}=570$ $\mathrm{nm}$ for cumulative X-ray doses ranging from $18 \mathrm{~Gy}$ to $5200 \mathrm{~Gy}$. The arrow indicates the trend for increased X-ray doses. Inset: PL intensity over time where $\lambda_{\mathrm{ex}}=566 \mathrm{~nm}, \lambda_{\mathrm{em}}$ $=670 \mathrm{~nm}$. (c) Integrated RPL intensities of the excitation spectra from $550 \mathrm{~nm}$ to $650 \mathrm{~nm}$ as a function of cumulative X-ray dose. (d) Integrated RPL intensities of the emission spectra from $700 \mathrm{~nm}$ to $775 \mathrm{~nm}$ as a function of cumulative X-ray dose. 
In order to establish the capabilities of the RPL in terms of radiation dose monitoring the integrated intensities of both the excitation and emission spectra were evaluated as a function of X-ray dose. The excitation spectra were integrated over the wavelength range $550 \mathrm{~nm}-650 \mathrm{~nm}$, corresponding to a mix of the $\mathrm{F} / \mathrm{Mn}$ and $\mathrm{F}_{3, \mathrm{Mn}}$ excitations. The resulting integrated RPL data are shown in figure 4.18 (c). The RPL was approximately linear up to $3000 \mathrm{~Gy}$, where deviations from linearity over this dose range were due primarily to uncertainties in the sample position in the spectrofluorometer. The RPL increased at a rate of $\sim 0.03 \% / \mathrm{Gy}$ over this region. The emission spectra were integrated over the wavelength range $700 \mathrm{~nm}-775 \mathrm{~nm}$, corresponding primarily to the F/Mn emission and where additional emissions were expected to be negligible. The integrated RPL data are shown in figure 4.18 (d). In this case, the data were linear up to at least $5200 \mathrm{~Gy}$ and increased at a rate of $0.02 \% / \mathrm{Gy}$.

\subsubsection{Optically Stimulated Luminescence}

In addition to the $\mathrm{F} / \mathrm{Mn} \mathrm{RPL}$, samples of $\mathrm{NaMgF}_{3}: \mathrm{Mn}$ exhibited OSL that emerged primarily due to the defects observed in the optical absorption, discussed in Section 4.3.1. The possibility of making both OSL and RPL measurements in a non-interfering manner and in the same near tissue-equivalent material provides a versatility uncommon to most luminescence-based dosimeter materials. To that end, the $\mathrm{NaMgF}_{3}: 0.5 \% \mathrm{Mn}$ sample was studied regarding the OSL properties.

The OSL stimulation spectrum for the sample after an X-ray dose of $180 \mathrm{~Gy}$ is shown in figure 4.19 (a), derived by subtracting the PL spectrum where $\lambda_{\mathrm{em}}=590 \mathrm{~nm}$ obtained before irradiation from that obtained after irradiation. The data could be fitted to a sum of 5 Gaussians that peaked at $3.06 \mathrm{eV}(405 \mathrm{~nm}), 3.36 \mathrm{eV}(369 \mathrm{~nm}), 3.79 \mathrm{eV}(327 \mathrm{~nm}), 4.43$ $\mathrm{eV}(280 \mathrm{~nm})$, and $5.06 \mathrm{eV}(245 \mathrm{~nm})$. These peaks correlated with those observed in the optical absorption (figure 4.16). The peak at $405 \mathrm{~nm}$ arose from $\mathrm{F}_{2}$-centres that did not appear in the absorption due to their low concentrations at the delivered doses. These centres likely did not contribute to the OSL emission, though were optically bleached at the stimulation energies used for OSL, and appeared in the spectrum due to the weak $\mathrm{F}_{2}$ centre emission that occurs near $530 \mathrm{~nm}$. All other peaks contributed to the OSL emission and were due to $\mathrm{H}_{\mathrm{A}}$-centres (369 $\mathrm{nm}$ and $\left.327 \mathrm{~nm}\right)$, F-centres $(280 \mathrm{~nm})$, and interstitials $(245 \mathrm{~nm})$. The OSL stimulation wavelength selected for the dose-dependence studies was $320 \mathrm{~nm}$ due to its overlap with the F-centre and main $\mathrm{H}_{\mathrm{A}}$-centre peaks and the fact that $320 \mathrm{~nm}$ does not directly correspond to any $\mathrm{Mn}^{2+} \mathrm{PL}$ excitations.

OSL appeared only from the non-distorted $\mathrm{Mn}^{2+}$ (inset to figure 4.19 (b)). A sample 

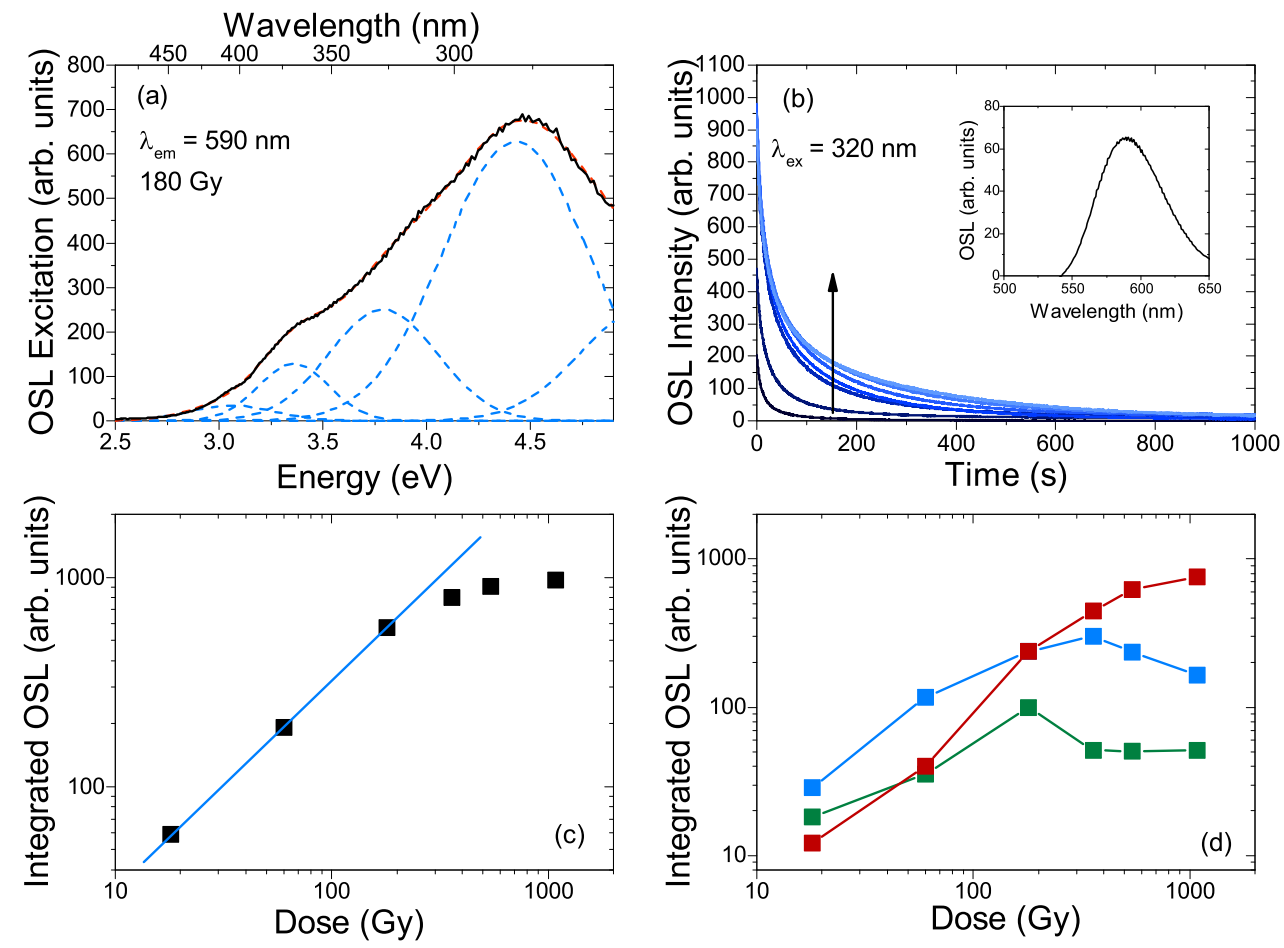

Figure 4.19: (a) OSL stimulation spectrum where $\lambda_{\mathrm{em}}=590 \mathrm{~nm}$, obtained by subtracting the PL excitation spectrum obtained prior to irradiation from the spectrum obtained after an X-ray dose of 180 Gy. Blue dashed lines are fitted Gaussian components and the red dashed line is the cumulative fit. (b) OSL decays where $\lambda_{\mathrm{ex}}=320 \mathrm{~nm}, \lambda_{\mathrm{em}}=$ $590 \mathrm{~nm}$, for X-ray doses ranging from $18 \mathrm{~Gy}$ to $1080 \mathrm{~Gy}$. The arrow indicates the trend for increased X-ray dose. Inset: OSL emission spectrum for an X-ray dose of $360 \mathrm{~Gy}$ and where $\lambda_{\mathrm{ex}}=397 \mathrm{~nm}$. (c) Integrated intensities of the OSL decays as determined by multi-exponential fitting, as a function of X-ray dose. (d) Integrated intensities of the individual exponential components, $A_{\mathrm{OSL}, 1}$ (green), $A_{\mathrm{OSL}, 2}$ (blue), and $A_{\mathrm{OSL}, 3}$ (red), as a function of X-ray dose. 
was irradiated with X-ray doses between 18 Gy and $1080 \mathrm{~Gy}$, then continuously stimulated with $320 \mathrm{~nm}$ light and the emission at $590 \mathrm{~nm}$ detected over time. A small residual PL component originating from the direct excitation of $\mathrm{Mn}^{2+}$ ions that was constant in time was subtracted from each OSL decay. Once the OSL was depleted, the sample was irradiated with a higher dose and the measurement repeated. The OSL decays for all doses are shown in figure 4.19 (c) and both the peak intensities and integrated intensities of the emissions increased with increasing X-ray dose.

All OSL decays could be fitted to the sum of 3 exponential decays, written

$$
I_{\mathrm{OSL}}(t)=A_{1} \times \exp \left(-t / \tau_{1}\right)+A_{2} \times \exp \left(-t / \tau_{2}\right)+A_{3} \times \exp \left(-t / \tau_{3}\right)
$$

where $I_{\mathrm{OSL}}(t)$ is the OSL intensity, $A_{i}$ are the exponential prefactors of the $i^{\text {th }}$ component, and $\tau_{i}$ are the decay constants for the $i^{\text {th }}$ component. The appearance of three distinct components was due to the stimulation wavelength $(320 \mathrm{~nm})$ overlapping with the three distinct OSL stimulation peaks that arose from traps with different concentrations, absorption cross-sections, and decay constants. The decay constants were $\tau_{1}=12.5 \mathrm{~s}, \tau_{2}$ $=79.8 \mathrm{~s}$, and $\tau_{3}=306 \mathrm{~s}$. The integrated intensities of the OSL decays were determined from the fitting as $A_{\mathrm{OSL}}=\sum_{i} A_{i} \tau_{i}$. $A_{\mathrm{OSL}}$ is plotted against radiation dose in figure 4.19 (c) and increased linearly up to at least $170 \mathrm{~Gy}$.

The integrated intensities of each OSL decay component, written $A_{\mathrm{OSL}, \mathrm{i}}=A_{i} \tau_{i}$, are shown in figure 4.19 (d) as a function of X-ray dose and are proportional to the total concentrations of the $i^{\text {th }}$ defects. $A_{\mathrm{OSL}, 1}$ increased up to $180 \mathrm{~Gy}$, after which it decreased to a constant value for higher doses. AOSL,2 increased up to 360 Gy before decreasing for higher doses. $A_{\mathrm{OSL}, 3}$ increased for higher doses, though appeared to be approaching saturation for doses above $1 \mathrm{kGy}$. The behaviour of $A_{\mathrm{OSL}, 1}$ and $A_{\mathrm{OSL}, 2}$ suggested that the concentrations of these particular OSL-active defects saturated for higher doses, where the decreases post-saturation occurred due to the competing $A_{\mathrm{OSL}, 3}$ excitation, and the possible aggregation of defects leading to different absorption cross-sections. Based on this behaviour and the respective decay constants for the stimulation wavelength of 320 nm, the components $A_{\mathrm{OSL}, 1}, A_{\mathrm{OSL}, 2}$, and $A_{\mathrm{OSL}, 3}$ were attributed to F-centres, $\mathrm{H}_{\mathrm{A}}$-centres $(327 \mathrm{~nm})$, and $\mathrm{H}_{\mathrm{A}}$-centres $(369 \mathrm{~nm})$, respectively. Saturation of the defect concentrations correlated with the saturation of the integrated OSL for doses above $180 \mathrm{~Gy}$.

The minimum detectable dose, defined as the dose for which $A_{\mathrm{OSL}}(D)$ is equivalent to three standard deviations of the background signal, was calculated to be $1.3 \mathrm{mGy}$ when using the spectrometer for measurements. 


\subsubsection{Radioluminescence}

In addition to RPL and OSL, $\mathrm{NaMgF}_{3}: \mathrm{Mn}$ exhibited $\mathrm{RL}$ during X-ray stimulation in the form of $\mathrm{Mn}^{2+}$ luminescence. The normalised RL emission spectra for all studied Mn concentrations are shown in figure 4.20 (a), where two peaks corresponding to the distorted and non-distorted $\mathrm{Mn}^{2+}$ were observed. No additional emissions were observed, indicating that the additional defect sites seen in the PL were not RL-active. The RL emissions followed a similar trend to the PL (figure 4.17 (a)) regarding the Mn concentrations and the ratio of the two $\mathrm{Mn}^{2+}$ emissions. As the Mn concentration was increased, the emission from the non-distorted $\mathrm{Mn}^{2+}$ sites increased relative to that of the distorted $\mathrm{Mn}^{2+}$ sites, in accordance with the expected saturation of the distorted site concentrations for higher total $\mathrm{Mn}^{2+}$ concentrations.
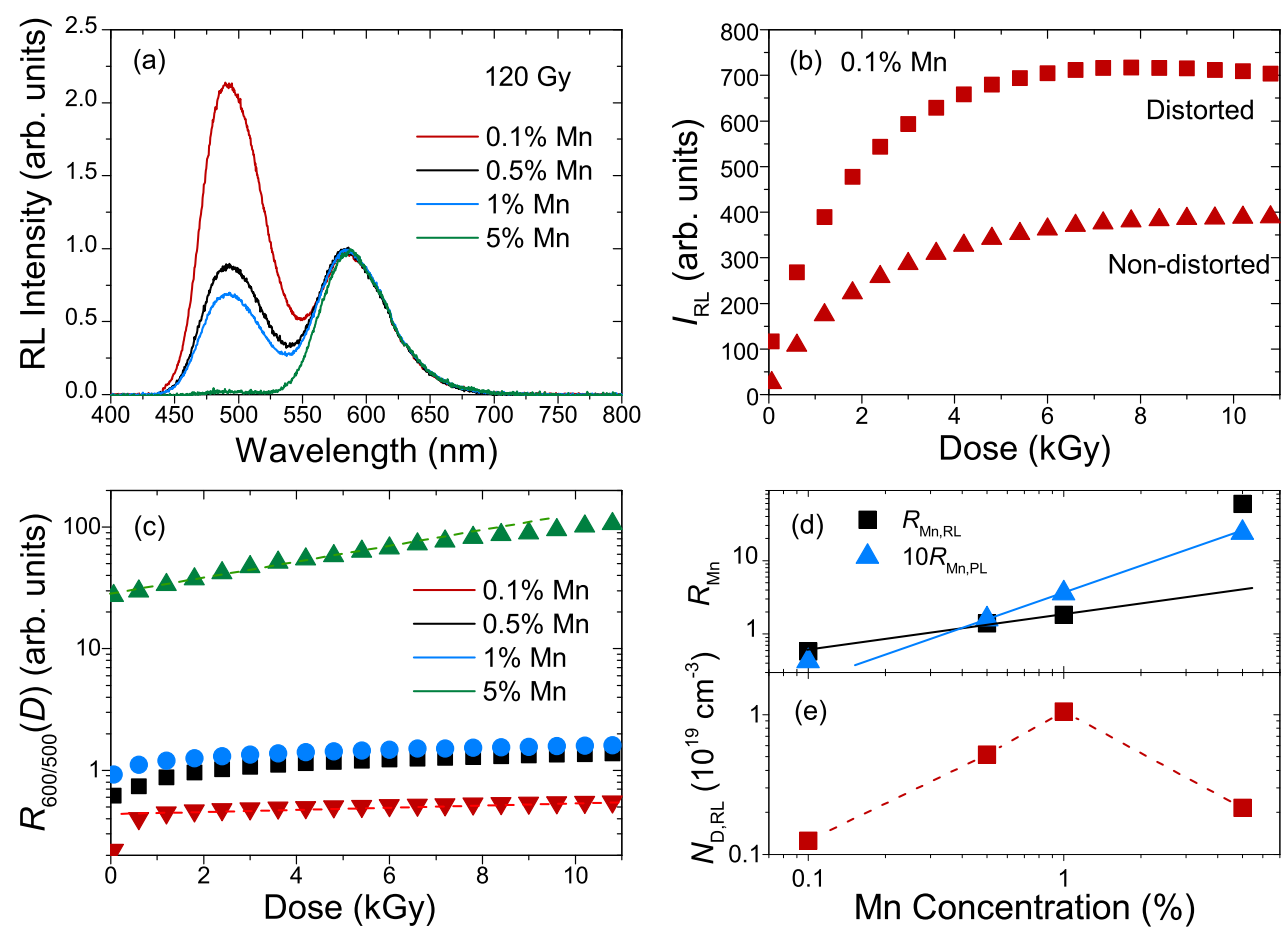

Figure 4.20: (a) RL emission spectra after a total X-ray dose of 120 Gy for Mn concentrations $0.1 \%$ (red), $0.5 \%$ (black), $1 \%$ (blue), and $5 \%$ (green). Spectra were normalised to the intensity of the non-distorted site at $590 \mathrm{~nm}$. (b) Integrated intensities of RL from the distorted sites (squares) and non-distorted sites (triangles) for $0.1 \% \mathrm{Mn}$. (c) $R_{600 / 500}(D)$ for Mn concentrations 0.1\% (red), 0.5\% (black), 1\% (blue), and 5\% (green). Dashed lines are linear fits to the data. (d) Ratio of the RL (black squares) and PL (blue triangles) emissions from the non-distorted and distorted Mn sites. Solid lines are guides to the eye. (e) Concentration of RL active distorted Mn sites as a function of total Mn concentration, as calculated using equation 4.5. 
The integrated RL emission intensities from each of the $\mathrm{Mn}^{2+}$ sites are shown in figure 4.20 (b) for the $0.1 \% \mathrm{Mn}$ sample. The emissions from both sites initially increased with increasing total dose and subsequently approached a saturation value at doses on the order of several kGy. The distorted site RL saturated at a lower dose than that of the non-distorted site.

The initial increase in RL intensity was due to a combination of mechanisms. In the first case, defects such as fluorine vacancies trapped those electrons produced during the irradiation period, and the trapping process competed with the recombination process that produced RL. As the trapping rate decreased as the traps were filled, the rate of RL recombination increased in response. This process saturated when the traps were all filled. The second phenomenon causing the increase was also related to trapping, and can be summarised as real-time OSL recombination [133]. Consider the OSL process: F-centres are stimulated into releasing their trapped electrons and the electrons then recombine with holes trapped elsewhere. The recombination energies are transferred to non-distorted $\mathrm{Mn}^{2+}$ ions and are released as photons. During X-ray stimulation, electron and hole traps are filled. However, free electrons and holes are also produced, and these may recombine via the OSL trapping sites. This leads to an additional emission component from $\mathrm{Mn}^{2+}$ that increases as the concentration of OSL-active defects increases, and where the traps should fill faster than they deplete such that OSL is still possible post-irradiation. This phenomenon was modeled in greater detail for the $\mathrm{NaMgF}_{3}: \mathrm{Sm}$ compound, as presented in Section 5.2.5.

It was of interest to analyse the ratio of the two $\mathrm{Mn}^{2+} \mathrm{RL}$ emissions as a function of dose and for each $\mathrm{Mn}^{2+}$ concentration. Practically, this could provide a means of monitoring radiation doses that is approximately independent of the collection optics used, as only the relative intensities of the two peaks would be required. The ratios of the integrated emissions of the non-distorted to distorted sites, $R_{600 / 500}(D)$, are presented in figure 4.20 (c) for the different $\mathrm{Mn}^{2+}$ concentrations. In all cases $R_{600 / 500}(D)$ increased with $\mathrm{X}$-ray dose, allowing for real-time total dose measurements after an initial characterisation. For $\mathrm{Mn} \leq 1 \%$ the $R_{600 / 500}(D)$ values behaved similarly, where there was an initial rise for low doses that approached a saturation for high doses. For $0.1 \% \mathrm{Mn}, R_{600 / 500}(D)$ increased at a rate of $\sim 2 \% / \mathrm{kGy}$ after the initial rise, and could therefore function as a real-time dosimeter after a priming dose, with well-described luminescence behaviour over time. For $5 \% \mathrm{Mn}$ the dose dependence of $R_{600 / 500}(D)$ was different, where the ratio increased linearly with dose up to approximately $9.6 \mathrm{kGy}$ at $26 \% / \mathrm{kGy}$. The linearity in this case suggests that the compound may be used for real-time dose measurements 
where the dose-history and total doses could be monitored via $R_{600 / 500}(D)$.

Figure 4.20 (d) shows the ratio of the non-distorted and distorted RL emissions at 120 Gy, $R_{\mathrm{Mn}, \mathrm{RL}}$, for the different Mn concentrations. As with the ratio of the PL emissions, $R_{\mathrm{Mn}, \mathrm{RL}}$ increased with $\mathrm{Mn}$ concentration. However, $R_{\mathrm{Mn}, \mathrm{RL}}$ was approximately 10 times greater than $R_{\mathrm{Mn}, \mathrm{PL}}$, indicating that a significant fraction of distorted $\mathrm{Mn}^{2+}$ sites did not participate in the RL recombination process. Figure 4.20 (e) shows the concentrations of distorted $\mathrm{Mn}^{2+}$ sites that contributed to the RL, calculated via

$$
N_{\mathrm{D}, \mathrm{RL}}=N_{\mathrm{N}, \mathrm{RL}} \tau_{\mathrm{D}} / R_{\mathrm{Mn}, \mathrm{RL}} \tau_{\mathrm{N}}
$$

and where $N_{\mathrm{N}, \mathrm{RL}}$ is the concentration of non-distorted sites that participated in the RL that was assumed to be equal to the $N_{\mathrm{D}}$ calculated from the PL. As such, the data are effectively normalised to the concentration of RL-active non-distorted sites. This analysis yielded that, at maximum, approximately $20 \%$ of the total concentration of distorted sites participated in the RL emissions for $1 \% \mathrm{Mn}$. The fraction decreased significantly for the $5 \% \mathrm{Mn}$ which was unexpected based on the saturation of distorted sites at high $\mathrm{Mn}$ concentrations. The decrease in the relative concentrations participating in the PL and RL would be expected if the defects responsible for site distortions also provided nonradiative recombination pathways nearby to the distorted $\mathrm{Mn}^{2+}$ sites, thus reducing the rates of RL recombination without affecting PL emissions. However, the decrease for $5 \% \mathrm{Mn}$ requires an alternative explanation, where this feature implies that there existed non-radiative recombination pathways that increased with Mn concentration.

\subsubsection{Summary and Discussion}

The above study of $\mathrm{NaMgF}_{3}: \mathrm{Mn}$ revealed both interesting optical features and promising dosimeter properties. Unexpected radiation-induced absorptions that peaked at $342 \mathrm{~nm}$ occurred that did not appear in non-doped $\mathrm{NaMgF}_{3}$ at $300 \mathrm{~K}$, though were detected at lower temperatures ( $80 \mathrm{~K})$ [130]. The $342 \mathrm{~nm}$ absorptions were attributed to hole traps, described as $\mathrm{H}_{\mathrm{A}}$-centres that were localised to $\mathrm{Mn}^{2+}$ impurities that functioned to stabilise the defects at room temperature. Additional radiation-induced absorptions were observed that were expected at room temperature, including OSL-active F-type centres. The concentrations of each defect decreased as the Mn concentrations increased, likely due to the additional $\mathrm{Mn}$ ions reducing charge trapping rates by increasing charge recombination rates.

$\mathrm{NaMgF}_{3}: \mathrm{Mn}$ was unique among the fluoroperovskites in that it exhibited two $\mathrm{Mn}^{2+}$ 
emissions where only one was expected. PL data revealed that emission from distorted $\mathrm{Mn}^{2+}$ dominated for low Mn concentrations, whereas emission from non-distorted $\mathrm{Mn}^{2+}$ dominated as the Mn concentration was increased. PL lifetime measurements were consistent with the distorted/non-distorted attributions and were used to estimate the concentrations of each $\mathrm{Mn}$ site as a function of total $\mathrm{Mn}$ concentration. It was determined that the distorted sites saturated for total Mn concentrations above 1\%, while the concentrations of non-distorted $\mathrm{Mn}^{2+}$ sites continued to increase with total Mn concentration. This suggested that there existed a limited concentration of available distorted $\mathrm{Mg}$ sites that could be substituted by Mn ions and that the sites were not explicitly related to the Mn doping.

The luminescence features of the F/Mn complexes were studied as a function of dose. It was demonstrated that the F/Mn emission could be non-destructively probed by exciting into the low-energy excitation bands of the complex and that the emission intensities increased linearly as a function of dose to over $5 \mathrm{kGy}$. This represents an extremely wide dose range over which RPL dose measurements may be performed. Furthermore, the non-destructive measurement technique should allow for the monitoring of large cumulative doses over extended time periods.

The compound additionally showed OSL after irradiation that arose from several types of radiation-induced defects. The OSL intensities when stimulated at $320 \mathrm{~nm}$ were linear up to approximately $180 \mathrm{~Gy}$. While this is a significantly smaller dose range than that seen for the RPL, saturation at 180 Gy remains a significant improvement over several commercial dosimeters where the linearity fails well below 100 Gy $[39,134]$.

Finally, RL was observed during continuous irradiation of the samples with emissions occurring only from the $\mathrm{Mn}^{2+}$ sites. Similar behaviour to the PL was observed for the distorted and non-distorted $\mathrm{Mn}^{2+}$ sites, though where fewer distorted sites appeared to participate in the RL process. This was likely due to the existence of non-radiative recombination pathways near these sites. RL from both sites increased during continuous X-ray exposure before saturating at doses above several $\mathrm{kGy}$. The ratio of the nondistorted to distorted emissions yielded an approximately linear parameter that could be used to monitor X-ray doses in real time, where the specific function of the dosimeter would determine the ideal Mn concentration.

In summary, the dosimetric properties of $\mathrm{NaMgF}_{3}: \mathrm{Mn}$ in terms of RPL, OSL, and RL were explored. The single material could monitor doses using all three luminescence phenomena and could therefore operate both actively and passively. Real-time measurements could be made using RL up to very high doses on the order of several kGy, 
though depending on the Mn concentration a well-defined priming dose would be required. The compound showed more promise as a passive dosimeter, where the RPL and OSL phenomena could be exploited together to improve dosimeter accuracy and versatility. Small cumulative doses could be probed using the non-destructive RPL, where the stimulation energy is too small to affect the OSL-active defects. The RPL could also be monitored in real-time, provided adequate optical filtering of the RL is achieved. Upon confirming the desired radiation dose via RPL monitoring, the dosimeter could be optically bleached at $320 \mathrm{~nm}$, during which a confirmation dose measurement would be made via OSL and the dosimeter would be entirely reset. Combined OSL/RPL dosimetry is not possible in most materials, indicating that the near tissue-equivalent $\mathrm{NaMgF}_{3}: \mathrm{Mn}$ is a very promising candidate for dosimetry.

\subsection{Dosimetric Properties of $\mathrm{CsCdF}_{3}: \mathrm{Mn}$}

The basic luminescence properties of $\mathrm{CsCdF}_{3}: \mathrm{Mn}$ presented in Section 4.1 revealed characteristics unique to the compound among the studied series of fluoroperovskites. A broad excitation band was observed in the UV $(\sim 250 \mathrm{~nm})$ that was not due to intraconfigurational $\mathrm{Mn}^{2+}$ excitations, but rather a charge transfer process. The smaller band gap of the compound ( $\sim 5.5 \mathrm{eV}$ [86]) relative to the $A M g F_{3}$ compounds ( $12 \mathrm{eV}$ [83-85]) suggested that the charge carrier kinetics, such as those cross-band gap electronic excitations that occur during exposure to ionising radiation, could be explored in detail using optical stimulation. Presented in this section is a study of the luminescence and radiation sensing properties of $\mathrm{CsCdF}_{3}: \mathrm{Mn}$, where it was observed that the compound may be useful for X-ray sensing, UV sensing, and optical information storage.

\subsubsection{UV Irradiation}

Figure 4.21 (a) shows a Tauc plot for $\mathrm{CsCdF}_{3}: \mathrm{Mn}$ prior to irradiation, where the product $(\alpha h v)^{2}$ is plotted against photon energy. Assuming that the optical transitions across the band gap were direct, the high-energy linear absorption component was extrapolated to the energy axis to yield a band gap energy of $\varepsilon_{g}=5.8 \mathrm{eV}(214 \mathrm{~nm})$, in close agreement with the previously determined value [86]. Band structure calculations for pure $\mathrm{CsCdF}_{3}$ produce band gap energies ranging from $\sim 4.2 \mathrm{eV}$ to $\sim 7 \mathrm{eV}[89,135]$, also in agreement. The calculations also predicted the existence of indirect band gaps at lower energies - however, the probabilities of indirect optical transitions are significantly lower than 
those of direct optical transitions, and therefore the direct transitions are the dominant mechanism by which cross-band luminescence phenomena are studied.
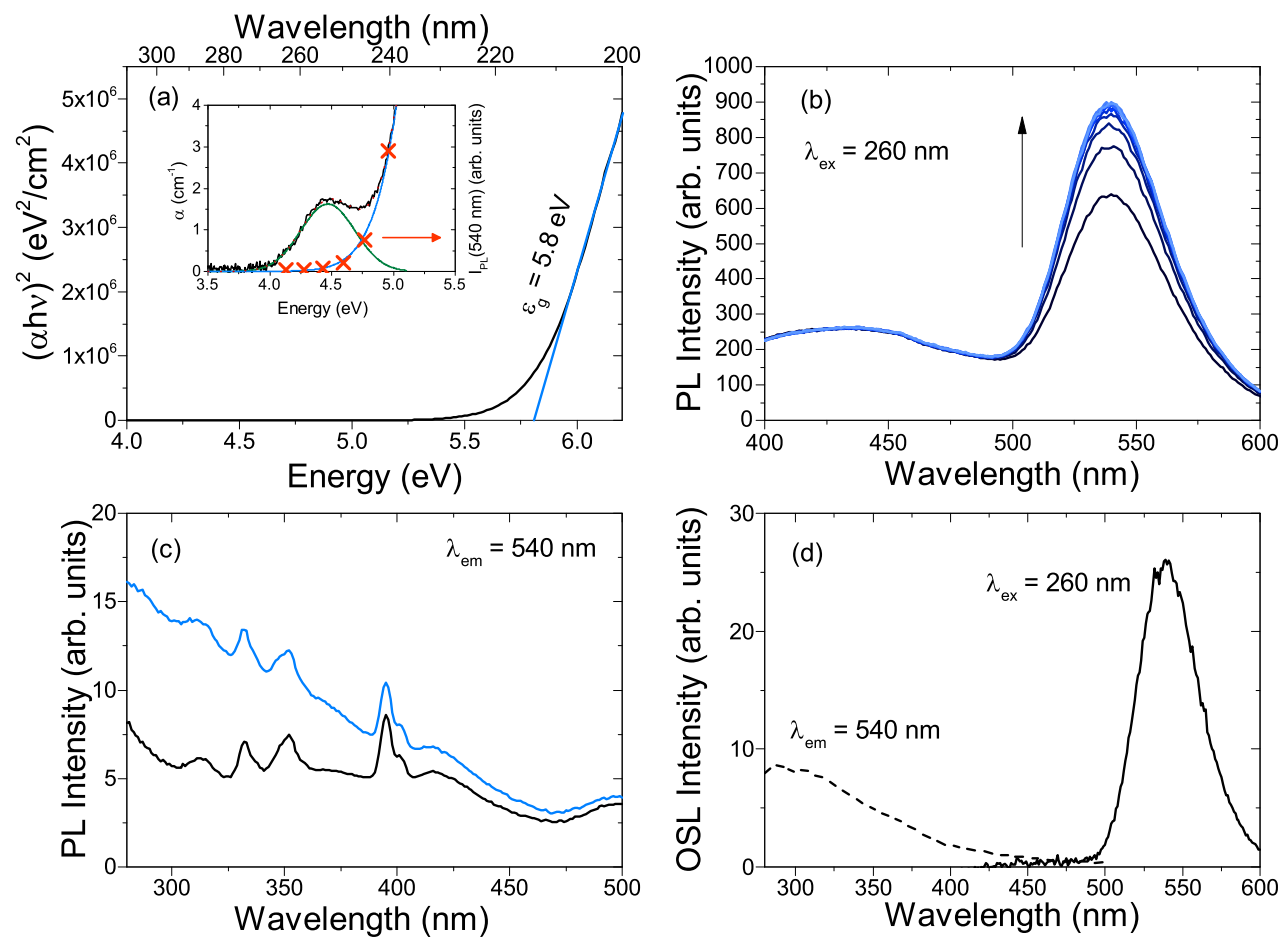

Figure 4.21: (a) Optical absorption expressed as $(\alpha h v)^{2}$ (black) and a linear extrapolation of the high energy component to the energy axis (blue). Inset: Optical absorption after irradiation at $254 \mathrm{~nm}$ (black). Gaussian fits corresponding to F-centres (green), the UV excitation band (blue), and the cumulative fit (red dashed) are also shown. The orange crosses correspond to the saturation values of $I_{f}(540, \lambda)$ after $600 \mathrm{~s}$ of stimulation at the relevant energies, scaled to the absorption data. (b) PL emission spectra where $\lambda_{\text {ex }}=260$ $\mathrm{nm}$ taken concurrently. The arrow indicates the trend for the concurrent scans. (c) PL excitation spectra where $\lambda_{\mathrm{em}}=540 \mathrm{~nm}$ after $260 \mathrm{~nm}$ stimulation (blue) and after complete optical bleaching of the sample (black). (d) OSL stimulation spectrum where $\lambda_{\mathrm{em}}=540$ $\mathrm{nm}$ (black dashed) and OSL emission spectrum where $\lambda_{\mathrm{ex}}=260 \mathrm{~nm}$ (black), obtained by taking the difference between spectra obtained before and after UV stimulation.

The inset to figure 4.21 (a) shows the absorption coefficient, $\alpha$, of the sample after irradiation with $254 \mathrm{~nm}$ light. A component was observed that peaked at approximately $4.4 \mathrm{eV}(281 \mathrm{~nm})$ that was due to the formation of F-centres [86]. While F-centre absorptions have been observed after X-ray irradiation in all samples studied in this work, the appearance of F-centres after UV stimulation occurred only for $\mathrm{CsCdF}_{3}: \mathrm{Mn}$. The band gap energy $(5.8 \mathrm{eV})$ is significantly higher than the $254 \mathrm{~nm}$ stimulation energy $(4.9 \mathrm{eV})$, excluding the possibility of cross-band gap excitations producing the necessary conduc- 
tion band electrons required for F-centre formation. This observation suggested that instead of cross-band excitations, a charge transfer process occurred, where electrons occupying states within the band gap were excited to the conduction band and subsequently trapped by fluorine vacancies. It is proposed that the initial state is the $\mathrm{Mn}^{2+}$ ground state, such that the process may be described by $\mathrm{Mn}^{2+}+h v \rightarrow \mathrm{Mn}^{3+}+\mathrm{e}^{-}$. This model satisfactorily describes the two major effects of UV stimulation: (i) the free electron, $\mathrm{e}^{-}$, occupies the conduction band and may be trapped to form F-centres, and (ii) the inverse process, where the free electron recombines with $\mathrm{Mn}^{3+}$ such that $\mathrm{Mn}^{3+}+$ $\mathrm{e}^{-} \rightarrow h v+\mathrm{Mn}^{2+}$, produces $\mathrm{Mn}^{2+}$ emissions of high intensity. The high intensity of the $\mathrm{Mn}^{2+}$ emission was due to the fact that the charge transfer transition is neither spin nor parity forbidden, unlike the $3 d \rightarrow 3 d$ transitions of $\mathrm{Mn}^{2+}$. Consequently, charge transfer phenomena can be exploited to produce strong PL emissions using ions with low oscillator strengths.

Figure 4.21 (b) illustrates the effect of concurrent $\lambda_{\mathrm{ex}}=260 \mathrm{~nm}$ excitations on the $\mathrm{Mn}^{2+}$ PL emission $\left({ }^{4} \mathrm{~T}_{1}(\mathrm{G}) \rightarrow{ }^{6} \mathrm{~A}_{1}(\mathrm{~S})\right)$ peaking at $540 \mathrm{~nm}$. Each spectrum corresponded to $100 \mathrm{~s}$ of continuous stimulation. The weak $\mathrm{O}^{2-}$-related emission was unaffected by the UV stimulation, whereas the $\mathrm{Mn}^{2+}$ emission increased for subsequent scans before saturating after approximately $300 \mathrm{~s}$ of exposure. The peak emission intensity increased by approximately $140 \%$ over the entire stimulation period. This increase is not welldescribed by the charge transfer model if F-centres are the only electron trap. However, the introduction of an additional shallow electron trap to the model successfully describes the PL enhancement and will be discussed in detail shortly.

Figure 4.21 (c) shows the PL excitation spectra obtained after $254 \mathrm{~nm}$ stimulation and after bleaching of the sample using $300 \mathrm{~nm}$ - $500 \mathrm{~nm}$ light. It is clear that the UV stimulation produced a broad excitation band at low wavelengths and that was superimposed on the $\mathrm{Mn}^{2+} 3 d \rightarrow 3 d$ excitations. The background was due to the UV-induced production of OSL-active F-centres, such that stimulation into the band produced $\mathrm{Mn}^{2+}$ emission that decayed during continuous stimulation. The OSL stimulation and emission spectra are shown in figure 4.21 (d), obtained by subtracting the spectra taken before UV stimulation from those taken after UV stimulation. The stimulation curve peaked at $287 \mathrm{~nm}$, corresponding to the F-centre band per the optical absorption. The OSL emission peaked at $540 \mathrm{~nm}$, corresponding to the $\mathrm{Mn}^{2+}$ emission, as expected.

The previously discussed data revealed that $\mathrm{CsCdF}_{3}: \mathrm{Mn}$ could be optically charged with UV light $(254 \mathrm{~nm})$ such that the $\mathrm{Mn}^{2+}$ emissions were enhanced and OSL-active defects were produced. The charging, afterglow, and OSL effects are characterised by 


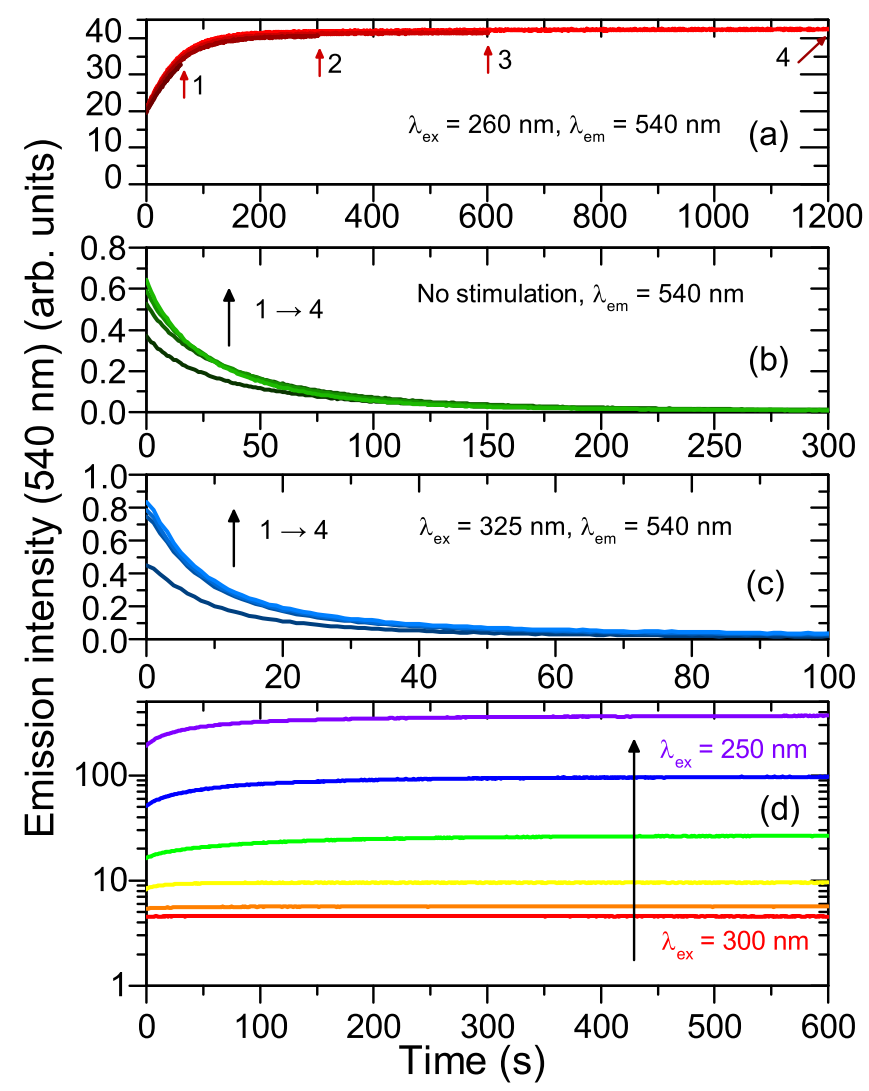

Figure 4.22: (a) $\mathrm{Mn}^{2+}$ PL emissions where $\lambda_{\mathrm{em}}=540 \mathrm{~nm}$ as a function of UV stimulation time where $\lambda_{\text {ex }}=260 \mathrm{~nm}$, and where the arrows indicate the total stimulation (charging) times of (1) 1 minute, (2) 5 minutes, (3) 10 minutes, and (4) 20 minutes. (b) $\mathrm{Mn}^{2+}$ afterglow emissions where $\lambda_{\mathrm{em}}=540 \mathrm{~nm}$ in the absence of any stimulation, after the charging described in (a). The arrow shows the trend for increased charge times. (c) $\mathrm{Mn}^{2+}$ OSL emissions where $\lambda_{\mathrm{ex}}=325 \mathrm{~nm}, \lambda_{\mathrm{em}}=540 \mathrm{~nm}$, taken after the charging described in (a) and after the afterglow had decayed fully. The arrow shows the trend for increased charge times. (d) $\mathrm{Mn}^{2+}$ PL emissions where $\lambda_{\mathrm{em}}=540 \mathrm{~nm}$ for different stimulation wavelengths between $300 \mathrm{~nm}$ and $250 \mathrm{~nm}$ (10 $\mathrm{nm}$ increments). The arrow indicates the effect of decreasing the stimulation wavelength. 
figure 4.22. In all cases, emissions from $\mathrm{Mn}^{2+}$ were monitored, where $\lambda_{\mathrm{em}}=540 \mathrm{~nm}$. The sample was initially stimulated with UV light $(260 \mathrm{~nm})$ for $X$ minutes. The stimulation was then ceased and the emission monitored as a function of time after a $10 \mathrm{~s}$ delay and for at least $300 \mathrm{~s}$. Finally, the sample was stimulated with $325 \mathrm{~nm}$ light and the OSL decay curves monitored. The entire process was repeated for different charging times, where $X=1$ minute, 5 minutes, 10 minutes, and 20 minutes.

Figure 4.22 (a) shows the effects of continuous charging on the $\mathrm{Mn}^{2+}$ emission. As expected per the PL spectra, the $\mathrm{Mn}^{2+}$ emission increased with longer UV stimulation times. The enhancement was rapid over the first $\sim 200 \mathrm{~s}$ and weakly linear for longer charge times. Figure 4.22 (b) shows the afterglow produced by the charging, where the sample continued to glow in the absence of any external stimulation for several hundred seconds. It is worth noting that the afterglow could be seen by eye for the first $\sim 60$ s. Figure 4.22 (c) shows the OSL decays for the different charge times, where readout occurred rapidly over less than $100 \mathrm{~s}$. It is clear that both the afterglow and OSL intensities saturated with the initial $\mathrm{Mn}^{2+}$ emission enhancement, indicating that all processes were proportional to one another and therefore dependent on similar physical phenomena. This was expected for the charging and afterglow data, as the initial $\mathrm{Mn}^{2+}$ enhancement should occur entirely due to the filling of shallow traps and the subsequent room temperature detrapping and $\left(\mathrm{Mn}^{3+}+\mathrm{e}^{-}\right)$recombination. The OSL depended instead on the existence of stable F-centres. A simple test of the OSL stability was performed and the OSL-active F-centres were found to be stable for at least 16 hours.

Figure 4.22 (d) illustrates the effect of different stimulation wavelengths on the $\mathrm{Mn}^{2+}$ PL, where $\lambda_{\text {ex }}$ was varied between $250 \mathrm{~nm}$ and $300 \mathrm{~nm}$. In all cases the initial $\mathrm{Mn}^{2+}$ intensity, $I_{0}(540, \lambda)$, the final $\mathrm{Mn}^{2+}$ intensity after $600 \mathrm{~s}$ of stimulation, $I_{f}(540, \lambda)$, and the difference, $I_{\text {incr }}(540, \lambda)=I_{f}(540, \lambda)-I_{0}(540, \lambda)$, increased for decreasing stimulation wavelengths. The data were entirely consistent with the excitation spectra (see figure 4.7), where $\lambda_{\mathrm{ex}}=300 \mathrm{~nm}$ corresponded to an excitation in the low energy tail of the charge transfer band such that the $\mathrm{Mn}^{2+} \mathrm{PL}$ enhancement was only $0.1 \%$. In contrast, $\lambda_{\text {ex }}=250 \mathrm{~nm}$ was toward the high energy peak of the charge transfer band and the $\mathrm{Mn}^{2+}$ enhancement was $94 \%$. These increases also correlated with the optical absorption, where the saturation values $I_{f}(540, \lambda)$ are plotted against the absorption in figure 4.21 (a). It should be noted that the peak intensity of the charge transfer band could not be resolved using the spectrometer due to its limited excitation range, though it is expected that by reducing the stimulation wavelength further a more significant enhancement of the $\mathrm{Mn}^{2+}$ emissions would be observed. 


\subsubsection{X-ray Irradiation}

The effects of X-ray irradiation on the $\mathrm{Mn}^{2+} \mathrm{PL}$ excitations are shown in figure 4.23. Xray exposure induced a broad excitation at low wavelengths that increased with increasing X-ray dose and the broad excitation was analogous to that induced by UV exposure (see figure 4.21 (c)). The sample was optically bleached between irradiations, such that each spectrum corresponds to a single dose ranging from $10 \mathrm{~Gy}$ to $100 \mathrm{~Gy}$. A single high energy X-ray photon excites multiple electrons from the valence band to the conduction band. The major point of difference between the X-ray and UV stimulations relates to the initial positions of the excited electrons relative to the host bands.

The OSL excitation curve is shown in the inset to figure 4.23 and peaked at $304 \mathrm{~nm}$. This was somewhat shifted when compared to the OSL peak induced by UV exposure $(287 \mathrm{~nm})$, though the characteristics of each curve were consistent and therefore the Xray induced peak was likely due to similar F-centre production. The intensities of the OSL stimulation curves taken after X-ray irradiation exceeded the maximum intensities achieved after UV stimulation, suggesting that significantly higher concentrations of Fcentres were produced by the X-ray irradiations. This suggests that either new fluorine vacancies were created and filled by the X-ray exposures, or alternatively that only a fraction of the pre-existing fluorine vacancies were accessible during UV stimulation. This was tested by exploring the effect of X-ray irradiation on the UV-induced charging of the OSL. If additional F-centres were created by the X-ray irradiations, the maximum OSL intensity observed after UV stimulation should correspondingly increase. However, X-ray irradiations had a negligible effect on the UV-induced OSL, suggesting that no significant concentrations of new vacancies were produced by the X-ray irradiations. It was concluded that only a fraction of fluorine vacancies can be filled by UV stimulation.

This phenomenon may be explained by considering that the $\mathrm{Mn}^{2+}$ levels are localised within the $\mathrm{CsCdF}_{3}$ band gap, whereas the valence band is continuous. The Mn concentration of the sample was nominally $0.2 \%$, such that the total Mn concentration was approximately $N_{\mathrm{Mn}}=2.2 \times 10^{19} \mathrm{~cm}^{-3}$. The F-centre concentration was estimated from the fitted data in figure 4.21 (a) and using equation 2.7, where the F-centre oscillator strength was assumed equal to 0.9 [55]. The total concentration of UV-induced F-centres was then $N_{\mathrm{F}}=6 \times 10^{15} \mathrm{~cm}^{-3}$, over three orders of magnitude lower than the Mn concentration. Based on these relative concentrations, we can consider that the $\mathrm{Mn}^{2+}$ electrons excited into the conduction band were more likely to recombine with a nearby $\mathrm{Mn}^{3+}$ ion than to be trapped by a fluorine vacancy, unless the vacancy was located nearby the excited $\mathrm{Mn}^{2+}$ ion. As the vacancies nearby $\mathrm{Mn}^{2+}$ ions were filled, the probability 


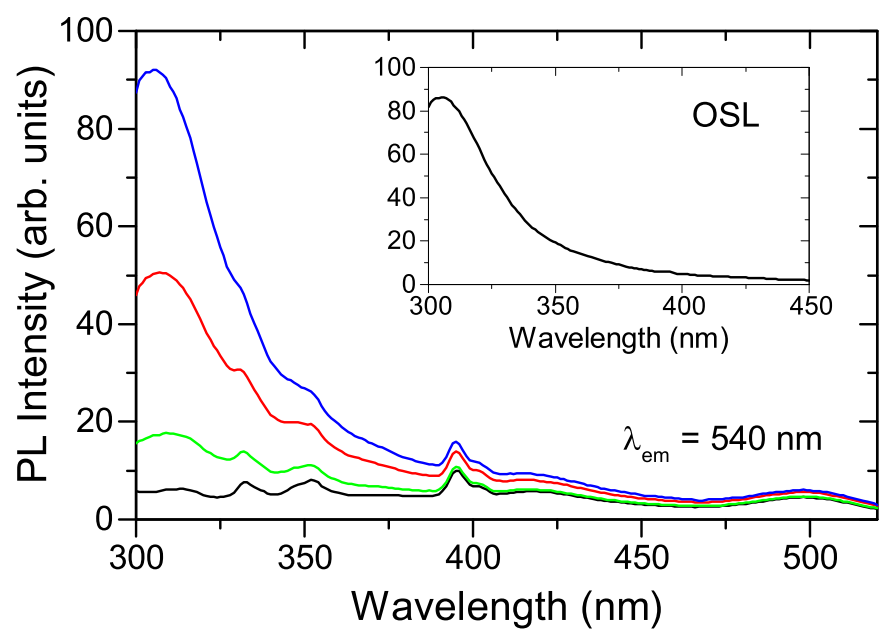

Figure 4.23: (a) PL excitation spectra where $\lambda_{\mathrm{em}}=540 \mathrm{~nm}$ prior to irradiation (black), and after X-ray doses of $10 \mathrm{~Gy}$ (green), $50 \mathrm{~Gy}$ (red), and $100 \mathrm{~Gy}$ (blue). Inset: OSL excitation spectrum obtained by subtracting the PL spectrum prior to irradiation from that taken after a 100 Gy dose.

of $\mathrm{Mn}^{3+}$ recombination further increased, such that the concentration of UV-induced Fcentres saturated rapidly. X-ray-induced conduction band electrons were not limited in the same way, where the surplus of excited electrons were not localised to $\mathrm{Mn}^{2+}$ ions and thus additional fluorine vacancies were filled throughout the irradiation process.

\subsubsection{Radioluminescence}

The RL spectra of $\mathrm{CsCdF}_{3}: \mathrm{Mn}$ are shown in figure 4.24 (a). RL emission occurred solely via the $\mathrm{Mn}^{2+}$ site throughout the two hours of X-ray exposure, indicating that $\mathrm{Mn}^{2+}$ was the only available cross-band gap radiative recombination centre. The dose dependence of the integrated RL intensity, $I_{\mathrm{RL}}(D)$, is shown in figure $4.24(\mathrm{~b}) . I_{\mathrm{RL}}(D)$ initially increased rapidly over the first $\sim 300$ Gy to $107 \%$ of the initial value, before adopting exponential behaviour well-described by an equation of the form

$$
I_{\mathrm{RL}}(D)=I_{\mathrm{RL}, \max }-A \exp (-D / \tau)
$$

where $I_{\mathrm{RL}, \max }$ is the saturation value of the integrated intensity at high dose, $A$ is an exponential prefactor, and $\tau$ is the decay constant expressed in kGy. The data were fitted to this equation, yielding the parameters $I_{\mathrm{RL}, \max }=119 \%, A=12.6 \%$, and $\tau=3.39$ kGy.

The initial increase of $I_{\mathrm{RL}}(D)$ was due to the rapid filling of fluorine vacancies that 

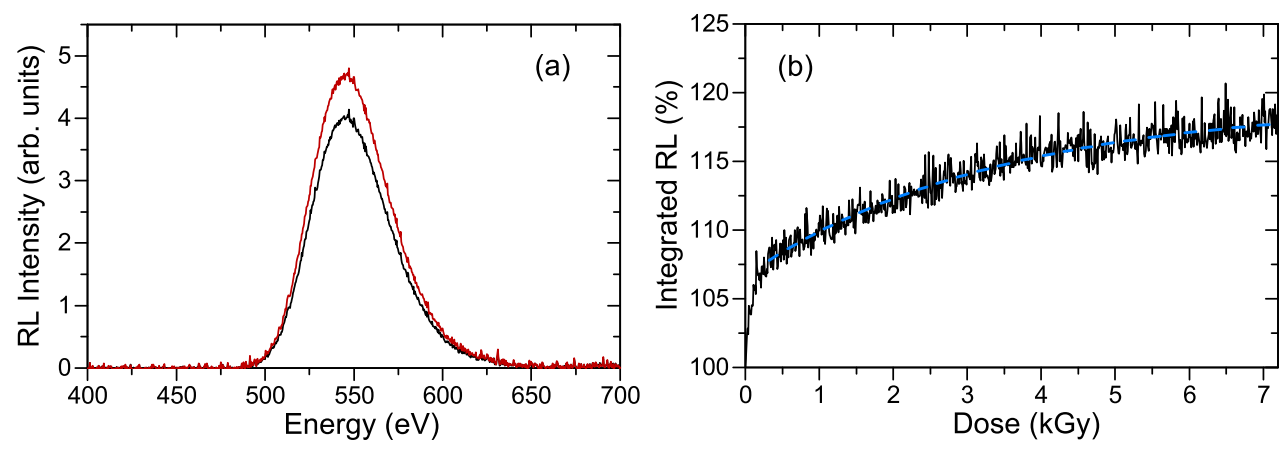

Figure 4.24: (a) RL emission spectra after 10 minutes of X-ray stimulation (black) and after 2 hours of stimulation (red) at approximately $1 \mathrm{Gys}^{-1}$. (b) Integrated intensity of the RL emission as a function of X-ray dose, normalised to the initial intensity. The blue dashed line is an exponential fit to the data.

initially acted to suppress the probability of recombination via $\mathrm{Mn}^{2+}$. The following slower increase was likely due to the occurrence of real-time OSL recombination, where free electrons produced during irradiation recombined with trapped holes and excited nearby $\mathrm{Mn}^{2+}$ ions. Following X-ray irradiation the sample exhibited an afterglow similar to that observed after UV charging, emphasising that shallow traps existed that emptied at room temperature.

Following the initial 300 Gy exposure, the intensity variations over smaller dose ranges ( $100 \mathrm{~Gy})$ were approximately linear, changing by only $0.004 \% / \mathrm{Gy}$. This nearinsensitivity to radiation dose history should allow for real-time dose measurements to be made using the compound when the doses of interest are in the $<100$ Gy region. Higher doses could be monitored after saturating the RL with a significantly higher priming dose i.e. once $I_{\mathrm{RL}}(D)=I_{\mathrm{RL}, \max }$ at $119 \%$.

\subsubsection{Thermoluminescence}

In order to better explore the deep and shallow traps in $\mathrm{CsCdF}_{3}: \mathrm{Mn}$, TL measurements were made at both low temperatures ( $230 \mathrm{~K}$ to $320 \mathrm{~K}$ ) and high temperatures ( $320 \mathrm{~K}$ to $540 \mathrm{~K})$, after both UV (254 nm) and X-ray irradiations. In all cases emission occurred solely via the $\mathrm{Mn}^{2+}$ recombination centre. The TL glow curves are shown in figure 4.25. Similar peaks appeared after the different irradiations with different intensities. Based on the previous discussions, we expected TL peaks below room temperature related to the afterglow, and TL peaks above room temperature related to the OSL. Peaks were observed over both temperature ranges and irradiation types, allowing for a compre- 
hensive analysis.
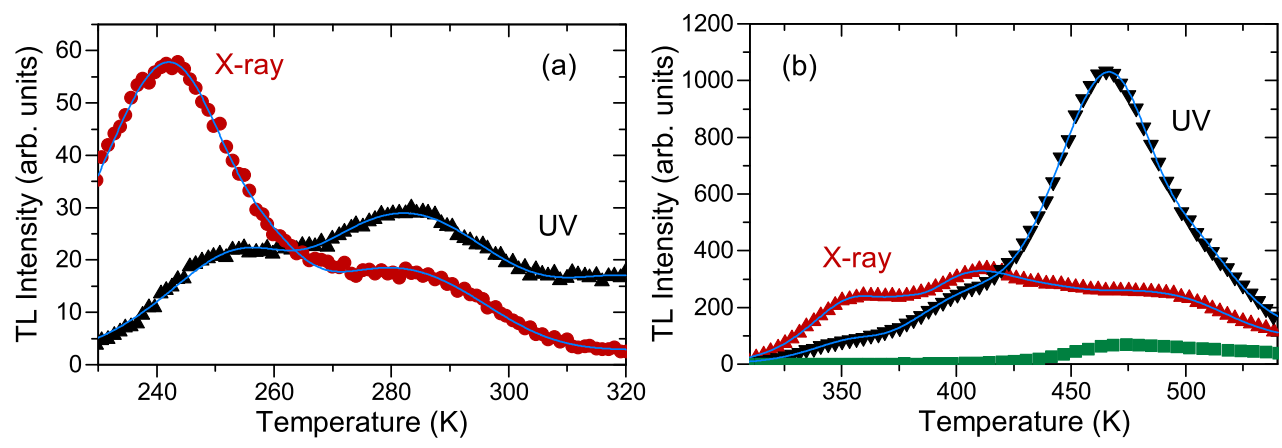

Figure 4.25: (a) TL glow curves obtained after an X-ray dose of 3 Gy (red circles) and after 1 hour of UV exposure (black triangles), below room temperature. Blue lines are fits to equation 4.6. (b) TL glow curves obtained after an X-ray dose of 3 Gy and a 10 minute delay (red circles), and after 1 hour of UV exposure and a 10 minute delay (black triangles), at room temperature. Blue lines are fits to equation 4.6. Also shown is the TL glow curve after a 3 Gy X-ray dose, a 10 minute delay, and 10 minutes of exposure to $365 \mathrm{~nm}$ light (green diamonds), at room temperature.

All glow curves were fitted to a sum of first-order kinetics TL peaks, written as [136]

$$
\begin{aligned}
I(T)=\sum_{i} I_{\mathrm{m}, i} \times & \exp \left(1+\left(E_{i} / k_{\mathrm{B}} T\right) \times\left(T-T_{\mathrm{m}, i}\right) / T_{\mathrm{m}, i}-T^{2} / T_{\mathrm{m}, i}^{2}\right) \\
& \times \exp \left(E_{i} / k_{\mathrm{B}} T \times\left(T-T_{\mathrm{m}, i}\right) / T_{\mathrm{m}, i} \times\left(1-2 k_{\mathrm{B}} T / E_{i}\right)-2 k_{\mathrm{B}} T_{\mathrm{m}, i} / E_{i}\right),
\end{aligned}
$$

where $I(T)$ is the TL intensity, $T$ is the temperature in $\mathrm{K}, I_{\mathrm{m}, i}$ is the peak intensity of the $i^{\text {th }}$ peak, $E_{i}$ is the trap depth of the $i^{\text {th }}$ peak, $T_{\mathrm{m}, i}$ is the temperature at which $I_{\mathrm{m}, i}$ occurs, and $k_{\mathrm{B}}$ is the Boltzmann constant. First order kinetics assumes negligible retrapping during heating [136] and applies to the majority of physical systems due to the typically large quantity of unique trapping levels [137]. The TL data, coupled with the previously discussed PL and OSL features, clearly demonstrate that there exists a number of different traps in the $\mathrm{CsCdF}_{3}: \mathrm{Mn}$ compound, justifying the adequacy of the first order fits in figure 4.25 .

After UV irradiation below room temperature ( 230 K) (figure 4.25 (a)), TL peaks occurred at $250 \mathrm{~K}, 279 \mathrm{~K}$, and $319 \mathrm{~K}$, with activation energies of $0.53 \mathrm{eV}, 0.37 \mathrm{eV}$, and 0.51 $\mathrm{eV}$, respectively. After X-ray irradiation at the same temperature similar peaks were fitted, though an additional component peaking at $239 \mathrm{~K}$ with an activation energy of 0.49 $\mathrm{eV}$ was observed. Based on the proximities to room temperature, the relative intensi- 
ties, and the activation energies, it was determined that the $279 \mathrm{~K}$ peak was most likely associated with the persistent luminescence/afterglow.

After UV irradiation at room temperature, and additionally after a 10 minute delay to avoid the detection of the low temperature TL (figure 4.25 (b)), TL components were fitted at $349 \mathrm{~K}, 396 \mathrm{~K}, 462 \mathrm{~K}, 485 \mathrm{~K}$, and $541 \mathrm{~K}$, with activation energies of $0.69 \mathrm{eV}, 0.59$ $\mathrm{eV}, 0.90 \mathrm{eV}, 0.62 \mathrm{eV}$, and $0.79 \mathrm{eV}$, respectively. After X-ray irradiation at room temperature and after a 10 minute delay, all peaks fitted to the UV TL were observed with the same activation energies, along with additional peaks at $371 \mathrm{~K}$ and $422 \mathrm{~K}$ with activation energies of $1.03 \mathrm{eV}$ and $0.52 \mathrm{eV}$, respectively.

The similar values of $T_{\mathrm{m}, i}$ and $E_{i}$ obtained across all TL spectra indicated that both $\mathrm{X}$-ray and UV irradiations produced a number of the same defects in the compound. The intensities of each component varied depending on the type of irradiation, expected based on the different charge kinetics for UV and X-ray excitations. As the UV stimulation was not a cross-band phenomenon, but rather a charge transfer process, the probabilities of charge trapping differed significantly from those encountered during Xray stimulation. Furthermore, all UV-induced peaks were observed in the X-ray glow curves, while the inverse was not true, demonstrating the difference in charge kinetics.

Finally, in order to test the relationship between the high temperature TL peaks and the OSL of the compound, a sample was irradiated with a dose of $3 \mathrm{~Gy}$, left for 10 minutes, then stimulated with $365 \mathrm{~nm}$ light. The OSL bleach erased a majority of the TL signal, indicating that a majority of the TL-active defects participated in the OSL process. There remained a small TL component at $541 \mathrm{~K}$ after the OSL bleach that was likely due to a defect with a lower optical stimulation probability than those producing the other peaks, and which likely did not participate as actively in the OSL process.

\subsubsection{Summary and Discussion}

$\mathrm{CsCdF}_{3}: \mathrm{Mn}$ was unique among the studied Mn-doped fluoroperovskites as its band gap energy was significantly lower than those of the $A \mathrm{MgF}_{3}$ compounds. This property manifested several interesting luminescence features, most prominently as charge transfer phenomena at UV stimulation energies. Based on optical absorption data, the band gap of the compound was measured to be $5.8 \mathrm{eV}$. However, it was subsequently observed that after stimulating the sample with $254 \mathrm{~nm}$ light $(4.9 \mathrm{eV})$ F-centres were produced, suggesting that sub-band gap energies were capable of producing conduction band electrons. Based on the intense $\mathrm{Mn}^{2+}$ luminescence observed during stimulation into the charge transfer band, it was proposed that $\mathrm{Mn}^{2+}$ ion acted as an electron donor level within the 
band gap. Electrons could then be excited from the ground state to the conduction band using $254 \mathrm{~nm}$ light, written $\mathrm{Mn}^{2+}+h v(254 \mathrm{~nm}) \rightarrow \mathrm{Mn}^{3+}+\mathrm{e}^{-}$, and the free electron could either recombine with the $\mathrm{Mn}^{3+}$ ion to produced $\mathrm{Mn}^{2+}$ luminescence, or alternatively be captured in some electron trap.

The F-centres produced during UV stimulation could be stimulated at slightly lower energies $(\sim 300 \mathrm{~nm})$, upon which the trapped electrons were released and emission from $\mathrm{Mn}^{2+}$ was observed. The occurrence of OSL after UV stimulation could allow for the compound to function as a high-energy UV dosimeter, though the photon energy range over which accurate measurements could be made would be limited to $\lesssim 280 \mathrm{~nm}$ due to the overlap of the OSL stimulation energies. Perhaps more interestingly, the optical/UV induction of colour centres in the compound could be used to form novel optical structures, e.g. optically-switchable Bragg gratings [100], or for binary information storage based on charge transfer and fluorescence detection [138].

The charge kinetics were analysed as a function of stimulation time, whereupon it was observed that UV stimulation also induced persistent luminescence in the sample. The intensities of the afterglow luminescence and OSL were directly proportional to the initial UV stimulation time, and both reached their relative maxima at the same stimulation times. This suggested a relationship between the defects responsible for the OSL and the afterglow.

The effects of X-ray irradiation on the compound were studied to provide a comparison between the charge transfer kinetics and the cross-band kinetics. X-ray stimulation produced a greater number of OSL-active F-centres than the UV stimulation, though the mechanism of F-centre formation remained the same. Consequently, a localised model was adopted to explain the saturation of F-centres for UV stimulations, where only traps nearby to $\mathrm{Mn}^{2+}$ ions were accessible due to the statistical likelihood of recombination via $\mathrm{Mn}^{3+}$ sites rather than trapping in a vacancy. It is possible that the shallow traps responsible for the afterglow were F-centres adjacent to $\mathrm{Mn}$ ions, such that $\mathrm{Mn}^{3+}-\mathrm{F}_{\mathrm{Mn}^{-}}$ centre pairs were produced during UV irradiation that were short-lived and radiatively recombined to leave a $\mathrm{Mn}^{2+}-\mathrm{V}_{\mathrm{F}}$ pair. This simple model is presented schematically in figure 4.26, where the model qualitatively describes all charge kinetics related to electronic excitations from the $\mathrm{Mn}^{2+}$ ground state, and the observations of OSL and persistent luminescence.

$\mathrm{RL}$ measurements were made and the results indicated that $\mathrm{Mn}^{2+}$ was the only available cross-band recombination centre. Regarding applications, the real-time RL was nearly independent of dose history in the sub-kGy dose regime after an initial priming 


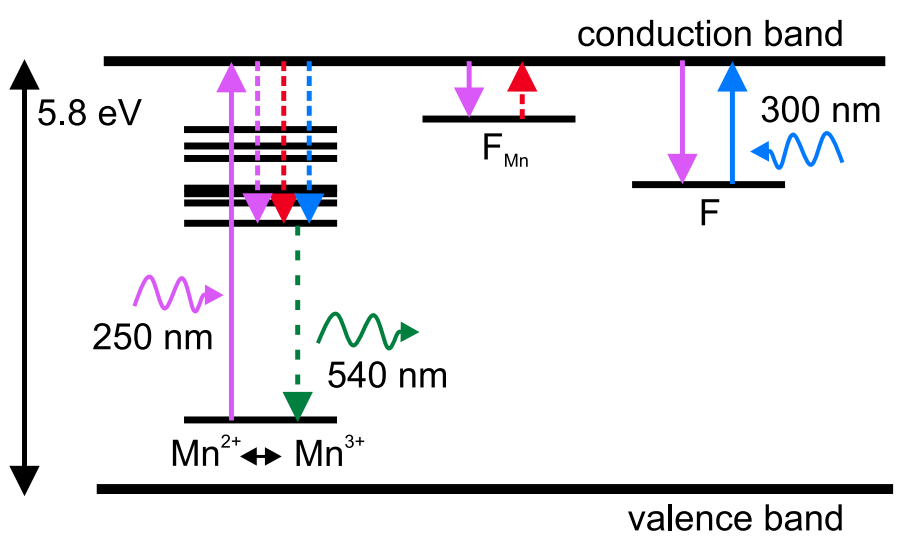

Figure 4.26: Simplified schematic of the charge carrier kinetics in $\mathrm{CsCdF}_{3}: \mathrm{Mn}$, illustrating the PL, OSL, and afterglow. Arrows correspond to the effects of $250 \mathrm{~nm}$ stimulation (violet), thermal detrapping (red), OSL stimulation (blue), and $\mathrm{Mn}^{2+}$ emission (green).

dose of $300 \mathrm{~Gy}$. Additionally, a priming dose on the order of $10 \mathrm{kGy}$ could be employed for higher dose rate measurements where applicable.

Finally, TL measurements were made on the compound and a number of traps were observed. Traps formed below room temperature were related to the persistent luminescence, and those formed above room temperature were related to the OSL. First order kinetics fitting of the curves established the peak temperatures and activation energies of each trap. The existence of multiple traps across a wide temperature range complicates the presented model regarding quantitative predictions, due to the distinct activation energies and stimulation probabilities of each defect.

Ultimately, a range of luminescence features were analysed for the compound $\mathrm{CsCdF}_{3}: \mathrm{Mn}$. The compound is not tissue-equivalent and is therefore unlikely to find applications in radiation dose monitoring. However, the optically-driven charge transfer and colour centre production could result in the eventual application of the compound in alternative fields, such as the production of photonic crystals or optical memory devices.

\subsection{Chapter Conclusions}

This chapter demonstrated several means through which radiation doses could be monitored using the compounds $\mathrm{NaMgF}_{3}: \mathrm{Mn}, \mathrm{KMgF}_{3}: \mathrm{Mn}, \mathrm{RbMgF}_{3}: \mathrm{Mn}$, and $\mathrm{CsCdF}_{3}: \mathrm{Mn}$. The $\mathrm{PL}$ of the $\mathrm{Mn}^{2+}$ ion was initially characterised for all host compounds. The emissions occurred via the ${ }^{4} \mathrm{~T}_{1}(\mathrm{G}) \rightarrow{ }^{6} \mathrm{~A}_{1}(\mathrm{~S})$ transition in all cases. The emission wavelength was dependent on the degree of crystal field splitting, which was in turn shown to be pro- 
portional to the Mn-F bond lengths via $10 D q \propto R_{\mathrm{Mn}-\mathrm{F}}^{-n}$ where $n=4.48 .10 D q$ values were calculated by fitting the $\mathrm{Mn}^{2+}$ excitation spectra and with reference to the TanabeSugano diagrams for $3 d^{5}$ ions in octahedral symmetries. The initial PL characterisations also revealed oxygen-related defects that produced a PL emission near $450 \mathrm{~nm}$ in all samples. Based on data obtained for various fluoride host compounds the defects were attributed to $\mathrm{O}^{2-}-\mathrm{V}_{\mathrm{F}}$ dipoles. Exposure to X-ray irradiation produced OSL in all samples that primarily arose from F-type centres. The observation of OSL postirradiation immediately indicated that all samples could function as passive OSL dosimeters, though where $\mathrm{NaMgF}_{3}: \mathrm{Mn}$ remained the most desirable compound due to its near tissue-equivalence.

F-centre/Mn complex PL was observed post-irradiation for the $A \mathrm{MgF}_{3}: \mathrm{Mn}$ complexes. It was of great interest to fully characterise the energies of these complexes for each compound in order to evaluate any potential trends and obtain some physical insight into the nature of the complexes. An emission-wavelength-dependent excitation spectra fitting technique was employed to evaluate the F/Mn energies for each compound. Ultimately, it was discovered that the F/Mn complexes energy levels were perturbed from the $\mathrm{Mn}^{2+}$ levels in all cases, where the F/Mn levels analogous to the crystal field split ${ }^{4} \mathrm{G}$ levels were reduced in energy relative to the ground states, and the analogous ${ }^{4} \mathrm{D}$ levels were increased in energy. This observation complicates the current theoretical interpretation of the complexes whereby spin-coupling of the $\mathrm{Mn}^{2+}$ ions and the F-centre electrons is assumed the primary mechanism by which the complexes form. The potential use of the F/Mn complexes in RPL dosimetry in various fluoride host compounds doped with Mn should be encouraged by this analysis and new theoretical calculations should be performed to better understand the underlying physics.

The near tissue-equivalence of $\mathrm{NaMgF}_{3}: \mathrm{Mn}$, coupled with the observations of OSL and RPL, motivated a more extensive characterisation of the compound. To that end, the optical absorption, PL, RPL, OSL, and RL were studied for $\mathrm{NaMgF}_{3}: \mathrm{Mn}$ doped with different concentrations of $\mathrm{Mn}$. The presence of $\mathrm{Mn}$ was found to stabilise $\mathrm{H}_{\mathrm{A}}$-centres at room temperature that exhibited both strong optical absorptions and contributed to the OSL. The concentrations of distorted $\mathrm{Mn}^{2+}$ sites were found to saturate at high $\mathrm{Mn}$ concentrations, such that non-distorted $\mathrm{Mn}^{2+}$ ions dominated the luminescence at high Mn concentrations.

For dosimeter applications, the RPL, OSL, and RL features were most important. These features were studied as a function of X-ray dose in order to assess the viability of the compound as a dosimeter material and establish the potential use-cases. 
The RPL arising from the production of F/Mn complexes was shown to be linear up to at least $5 \mathrm{kGy}$, representing an extremely wide dose range. The RPL could be probed non-destructively by exciting into the low-energy excitation bands of the complexes. Furthermore, the complexes could theoretically be probed real-time during irradiation, given sufficient optical filtering were employed.

The OSL arising from F-centre $+\mathrm{H}_{\mathrm{A}}$-centre stimulations was shown to be linear up to approximately $180 \mathrm{~Gy}$, also representing a wide dose range in comparison to current commercial OSL dosimeters. The disadvantages of OSL in this compound were largely related to the high energy photons required to stimulate $(\sim 320 \mathrm{~nm})$ and the fact that emission arose from the $\mathrm{Mn}^{2+}$ ion, such that some residual PL must be subtracted from the OSL signal. This was easily achievable in a laboratory setting but somewhat complicates a practical device. However, the fact that OSL measurements could be made cooperatively with RPL measurements improves device versatility and measurement accuracy. The fact that OSL arose from F-centre stimulation, during which electrons were released from the centres, means that OSL measurements necessarily erase the RPL due to the F/Mn complexes. To that end, cumulative doses could be monitored using RPL as a quality assurance tool, and optically resetting the material with UV stimulation would provide a final dose measurement via OSL.

RL occurred during X-ray stimulation where emissions occurred via both distorted and non-distorted $\mathrm{Mn}^{2+}$ sites. The ratios of the integrated intensities could be manipulated in order to produce linear behaviour in some cases, providing an additional means of monitoring radiation doses in real time. Consequently, it was established that $\mathrm{NaMgF}_{3}: \mathrm{Mn}$ is a promising dosimeter compound, where OSL, RPL, and RL may be used individually or together to reliably measure radiation doses over a very wide dose range.

$\mathrm{CsCdF}_{3}: \mathrm{Mn}$ proved to be an interesting material due primarily to its band gap $\varepsilon_{g}=$ $5.8 \mathrm{eV}$. It was established that upon UV stimulation $(\sim 254 \mathrm{~nm})$ electrons could be excited to the conduction band and become trapped in F-centres. The F-centres could then be stimulated into producing OSL. Additionally, persistent luminescence was observed after the UV stimulation that lasted several minutes. A simple model was developed to qualitatively describe these features, whereby UV stimulation excited electrons from the $\mathrm{Mn}^{2+}$ ground state to the conduction band. The electrons could then recombine, become trapped in F-centres, or become trapped in shallow traps localised to $\mathrm{Mn}^{2+}$ that were unstable at room temperature. The validity of this model was tested against the various real-time luminescence changes upon various stimulations and both low- and high-temperature TL measurements. Ultimately, $\mathrm{CsCdF}_{3}: \mathrm{Mn}$ was deemed an unlikely 
candidate for radiation dosimetry due to its high effective atomic number and prevalence of shallow traps. However, the compound could find alternative applications, where the optically-induced charge transfer could be used to inscribe information that could then be probed via OSL. The compound could also function as UVC dosimeter, where doses could be probed using OSL stimulation in the UVB region. 


\section{Chapter 5}

\section{Luminescence of the Lanthanides in $\mathrm{NaMgF}_{3}: \mathrm{Ln}$}

The series of lanthanides $(\mathrm{Ln})$, comprised of the elements $\mathrm{La}(Z=57)$ through $\mathrm{Lu}(Z$ $=71$ ), have long been of interest due to the unique properties that arise from partially filled $4 f$ shells. Ln-containing compounds have been exploited in the development of numerous technologies, including solid state lasers [5], electric motors [139], and storage phosphors $[6,66,140]$. Ln doped compounds have also proven viable materials for radiation detection and dose monitoring $[45,46,141,142]$, motivating similar studies on the Ln-doped fluoroperovskites.

A majority of studies on the luminescence of lanthanides have focused on the highly stable trivalent ions, i.e. $\mathrm{Ln}^{3+}$ ions [72]. Such studies led to the development of the Dieke diagrams [71] (see figure 2.9) that serve as valuable references when assigning optical transitions in any Ln-doped compound. Studies focused on the divalent lanthanides $\left(\mathrm{Ln}^{2+}\right)$ are less common due to their relative instability in most compounds [72]. The most stable divalent $\mathrm{Ln}$ are $\mathrm{Eu}^{2+}, \mathrm{Sm}^{2+}$, and $\mathrm{Yb}^{2+}$, all of which can be optically detected in several materials prior to any specific sample treatments [72]. Other divalent Ln, including $\mathrm{Nd}^{2+}, \mathrm{Dy}^{2+}, \mathrm{Tm}^{2+}$, and $\mathrm{Er}^{2+}$, may be observed in some compounds after particular treatments including X-ray irradiation [143]. When X-ray irradiation induces a valence change in a compound that in turn affects the luminescence properties, the compound can be used for radiation dose monitoring [18].

Presented in this chapter are luminescence studies of $\mathrm{NaMgF}_{3}: \mathrm{Ln}$ compounds where $\mathrm{Ln}=\mathrm{Sm}, \mathrm{Eu}, \mathrm{Dy}$, or $\mathrm{Yb}$. These ions were selected partially due to their potential stabilities in the divalent states, such that the $\mathrm{Ln}^{2+}$ ion could act as an electron trap and produce RPL features useful for radiation dose monitoring. Only the $\mathrm{NaMgF}_{3}$ host was studied 
in this chapter due to its near tissue-equivalence. Charge transfer bands (CTBs) were observed in some cases that allowed for the construction of the first host-referred binding energy (HRBE) diagram for the $\mathrm{NaMgF}_{3}$ : $\mathrm{Ln}$ compounds. Various RPL features were also observed and characterised. The data were encouraging regarding applications in dosimetry and the physical models developed are expected to be useful in future studies of the fluoroperovskites and other luminescent compounds.

\subsection{Photoluminescence of $\mathrm{NaMgF}_{3}: \operatorname{Ln}$}

Initially, polycrystalline samples of $\mathrm{NaMgF}_{3}: 0.05 \% \mathrm{Sm}, \mathrm{NaMgF}_{3}: 0.05 \%, \mathrm{NaMgF}_{3}: 0.7 \% \mathrm{Dy}$ and $\mathrm{NaMgF}_{3}: 0.2 \% \mathrm{Yb}$ were synthesised and investigated in terms of PL. The dopant concentrations for the initial characterisations were arbitrary at this stage as maintaining tissue-equivalence was not necessary outside of the dosimetric characterisations. The basic PL properties of the Ln dopants $(\mathrm{Ln}=\mathrm{Sm}, \mathrm{Eu}, \mathrm{Dy}$, and $\mathrm{Yb})$ were evaluated and the specific electronic transitions identified. Samples were exposed to X-ray irradiation and the PL analysed before and after the exposure. The PL data are presented in this section.

For all $\mathrm{NaMgF}_{3}: \mathrm{Ln}$ samples studied herein it was expected that the $\mathrm{Ln}^{2+/ 3+}$ ions substituted for the $\mathrm{Na}^{+}$site on the basis of ionic radii [81]. Consequently, the formation of charge compensating defects was expected, possibly as interstitial $\mathrm{F}^{-}$ions, $\mathrm{Na}$ vacancies, or impurity $\mathrm{O}^{2-}$ ions. Local symmetries have only a minor effect on the $4 f^{n}$ energy levels due to effective shielding from the outer $5 s$ and $5 p$ orbitals (see 2.2.11) and therefore the energy levels of each $4 f^{n} \longleftrightarrow 4 f^{n}$ transition should be comparable to the published data for similar compounds [71]. The positions of $5 d$ energy levels are strongly affected by the crystal field and may be useful in analysing the local environment of dopant ions [72]. The $4 f^{n} \longleftrightarrow 4 f^{n-1} 5 d^{1}$ transitions for the trivalent $\operatorname{Ln}^{3+}$ typically occur at high energies in the VUV spectral region [69, 84], well beyond the range of the spectrometer employed for this work. However, the $4 f^{n} \longleftrightarrow 4 f^{n-1} 5 d^{1}$ transitions for the divalent $\mathrm{Ln}^{2+}$ often occur at significantly lower energies in the optical spectral region. This property is useful in verifying the existence of $\mathrm{Ln}^{2+}$ in a compound and also in achieving high luminescence efficiencies, as $4 f \rightarrow 5 d$ transitions are not forbidden under parity as the $4 f \rightarrow 4 f$ transitions are.

It is of interest to elaborate on the nature of charge transfer (CT) transitions prior to the discussion of the data. In the context of lanthanides, CT transitions are understood as the transfer of a $2 p$ electron from a ligand (e.g. $\mathrm{O}^{2-}, \mathrm{F}^{-}$) to the partially filled $4 f^{n}$ shell of a $\mathrm{Ln}$ ion (e.g. $\left.\mathrm{Ln}^{3+}\right)$ [144]. Consequently, the valence of the $\mathrm{Ln}$ ion is briefly reduced 
$\left(\mathrm{Ln}^{3+}+\mathrm{e}_{\mathrm{CT}}^{-} \rightarrow \mathrm{Ln}^{2+}\right)$, and a hole remains bound to the donor ligand $\left(\mathrm{F}^{-} \rightarrow \mathrm{F}^{0}\right)[110,145]$. This configuration is typically unstable and the electron-hole pair rapidly recombines. The recombination energy is transferred to the $\mathrm{Ln}$ ion, leaving $\mathrm{Ln}^{3+}$ in an excited state that can then radiatively return to the ground state. CT transitions can be exploited to achieve high luminescence efficiencies as the transitions are entirely allowed [146, 147].

It is non-trivial to experimentally measure CT energies in many compounds. In fluoride hosts the large band gaps $(\sim 12 \mathrm{eV})$ lead to large CT energies $(\gtrsim 7 \mathrm{eV})$ that may overlap the various $4 f \rightarrow 5 d$ absorptions [84, 144]. It is possible to observe CT bands arising from impurities, e.g. $\mathrm{O}^{2-}$ ions, that will occur at significantly lower energies $[144,148]$. This effect was exploited to construct the HRBE diagram for $\mathrm{NaMgF}_{3}: \operatorname{Ln}$ using the methods developed by Dorenbos [67, 70, 110, 145, 149, 150].

\subsection{1 $\mathrm{NaMgF}_{3}: \mathrm{Sm}$}
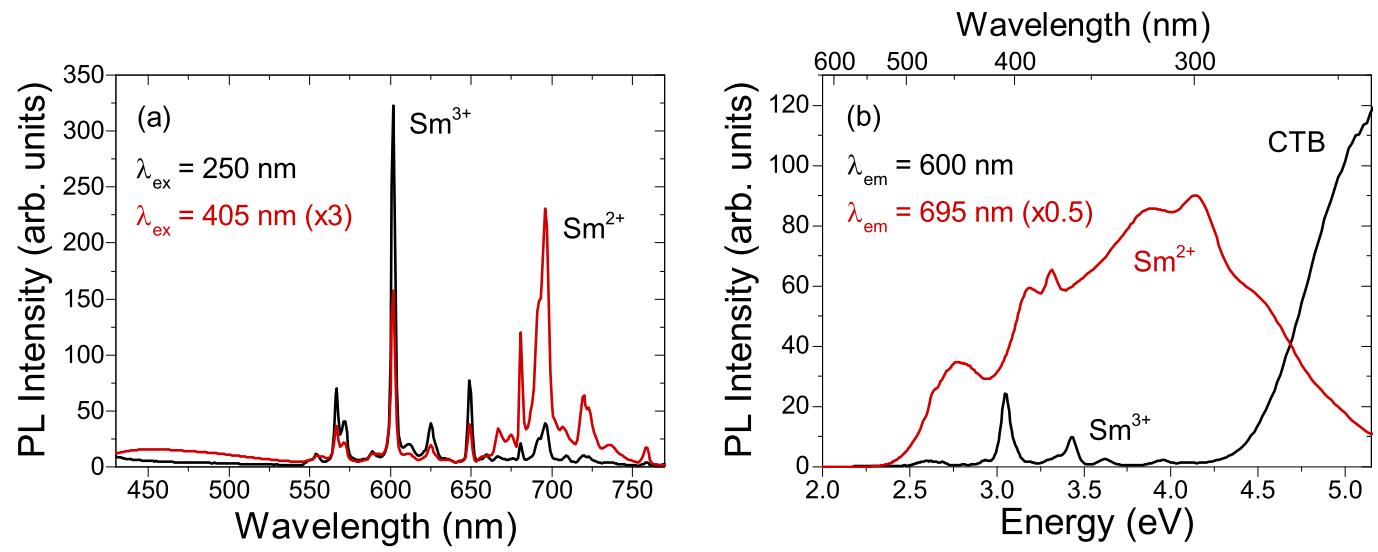

Figure 5.1: (a) PL emission spectra of $\mathrm{NaMgF}_{3}: \mathrm{Sm}$ where $\lambda_{\text {ex }}=250 \mathrm{~nm}$ (black) and $\lambda_{\text {ex }}=$ $405 \mathrm{~nm}(\times 3)(\mathrm{red})$. (b) PL excitation spectra of $\mathrm{NaMgF}_{3}: \mathrm{Sm}$ where $\lambda_{\mathrm{em}}=600 \mathrm{~nm}$ (black) and $\lambda_{\mathrm{em}}=695 \mathrm{~nm}(\times 0.5)(\mathrm{red})$.

The PL emission spectra of $\mathrm{NaMgF}_{3}: \mathrm{Sm}$ are shown in figure 5.1 (a) under the stimulation wavelengths $\lambda_{\mathrm{ex}}=250 \mathrm{~nm}$ and $\lambda_{\mathrm{ex}}=405 \mathrm{~nm}$. Both excitation wavelengths produced a range of sharp line emissions between $550 \mathrm{~nm}$ and $800 \mathrm{~nm}$ that were attributed to intraconfigurational $4 f \rightarrow 4 f$ transitions of both $\mathrm{Sm}^{3+}$ and $\mathrm{Sm}^{2+}$ ions, where those emissions between $550 \mathrm{~nm}$ and $650 \mathrm{~nm}$ were predominantly due to $\mathrm{Sm}^{3+}$, and those at longer wavelengths were due to $\mathrm{Sm}^{2+}$ (table 5.1). Under $405 \mathrm{~nm}$ stimulation a broad emission component occurred with a peak at approximately $450 \mathrm{~nm}$ that was attributed to trace oxygen (see Section 4.1.5). 


\begin{tabular}{cccc}
\hline $\mathrm{Sm}^{3+}$ & $\lambda(\mathrm{nm}) /$ Energy $(\mathrm{eV})$ & $\mathrm{Sm}^{2+}$ & $\lambda(\mathrm{nm}) /$ Energy $(\mathrm{eV})$ \\
\hline \hline${ }^{4} \mathrm{G}_{5 / 2} \rightarrow{ }^{6} \mathrm{H}_{9 / 2}$ & $649 / 1.91$ & ${ }^{5} \mathrm{D}_{0} \rightarrow{ }^{7} \mathrm{~F}_{3}$ & $760 / 1.63$ \\
\hline${ }^{4} \mathrm{G}_{7 / 2} \rightarrow{ }^{6} \mathrm{H}_{11 / 2}$ & $625 / 1.98$ & ${ }^{5} \mathrm{D}_{0} \rightarrow{ }^{7} \mathrm{~F}_{2}$ & $720 / 1.72$ \\
\hline${ }^{4} \mathrm{G}_{5 / 2} \rightarrow{ }^{6} \mathrm{H}_{7 / 2}$ & $602 / 2.06$ & ${ }^{5} \mathrm{D}_{0} \rightarrow{ }^{7} \mathrm{~F}_{1}$ & $696 / 1.78$ \\
\hline${ }^{4} \mathrm{G}_{5 / 2} \rightarrow{ }^{6} \mathrm{H}_{5 / 2}$ & $567 / 2.19$ & ${ }^{5} \mathrm{D}_{0} \rightarrow{ }^{7} \mathrm{~F}_{0}$ & $681 / 1.82$ \\
\hline
\end{tabular}

Table 5.1: Energies of the most intense transitions of $\mathrm{Sm}^{3+}$ and $\mathrm{Sm}^{2+}$ in $\mathrm{NaMgF}_{3}: \mathrm{Sm}$.

The excitation spectra corresponding to the peak emission wavelengths of each Sm valence, namely $\lambda_{\mathrm{em}}=600 \mathrm{~nm}\left(\mathrm{Sm}^{3+},{ }^{4} \mathrm{G}_{5 / 2} \rightarrow{ }^{6} \mathrm{H}_{7 / 2}\right)$ and $\lambda_{\mathrm{em}}=695 \mathrm{~nm}\left(\mathrm{Sm}^{2+},{ }^{5} \mathrm{D}_{0} \rightarrow{ }^{7} \mathrm{~F}_{1}\right)$, are shown in figure 5.1 (b). $\mathrm{Sm}^{3+}$ possessed several line excitations up to $4 \mathrm{eV}$ that were characteristic of $4 f^{5} \rightarrow 4 f^{5}$ excitations and were attributed as such. For energies greater than $4 \mathrm{eV}$ a broad excitation band onset that was characteristic of a CTB, though at an energy too low to arise from either $\mathrm{F}^{-}$excitations or excitations into the $5 d$ band of $\mathrm{Sm}^{3+}$. The CTB was therefore attributed to contaminant oxygen, where $\mathrm{O}^{2-}$ ions substituted for $\mathrm{F}^{-}$sites adjacent to the $\mathrm{Sm}^{3+}$ ions. The excitations of $\mathrm{Sm}^{2+}$ differed significantly from those of $\mathrm{Sm}^{3+}$, where several overlapping broad excitations were observed with weak line structures superimposed. The broad excitations were characteristic of divalent Ln and were due to excitations from the ${ }^{7} \mathrm{~F}_{\mathcal{f}}$ states to several $5 d$ levels, while the sharper components were due to transitions within the $4 f^{6}$ configuration. The greater intensity of the $\mathrm{Sm}^{2+} \mathrm{PL}$ was due largely to the increased oscillator strengths of the $4 f \rightarrow 5 d$ transitions rather than the relative concentrations of each ion. This will be demonstrated in Section 5.2.

Figure 5.2 shows the PL emissions where $\lambda_{\mathrm{ex}}=405 \mathrm{~nm}$ before and after an X-ray dose of $100 \mathrm{~Gy}$. The X-ray exposure resulted in a decrease in the $\mathrm{Sm}^{3+}$ emission intensity and an increase in the $\mathrm{Sm}^{2+}$ emission intensity. This was due to the radiation-induced valence conversion $\mathrm{Sm}^{3+}+\mathrm{e}^{-} \rightarrow \mathrm{Sm}^{2+}$. $\mathrm{Sm}^{3+}$ is a known electron trap in various compounds and glasses $[18,140,151,152]$, where the stability of the conversion depends on (i) the position of the $\mathrm{Sm}^{2+}$ ground state relative to the conduction band of the host compound, and (ii) the stability of the corresponding hole trap. In the fluorophosphate glasses it is understood that the hole is trapped on a phosphorous-oxygen hole centre [151], and the photoionisation of oxygen is also considered to be the physical mechanism by which $\mathrm{Sm}^{3+}$ reduces in other glasses and compounds $[140,152,153]$. Given the known presence of oxygen in $\mathrm{NaMgF}_{3}: \mathrm{Sm}$, as evidenced by the broad emission near $450 \mathrm{~nm}$ and the presence of a CTB, it was considered that a similar process takes place in the compound. 


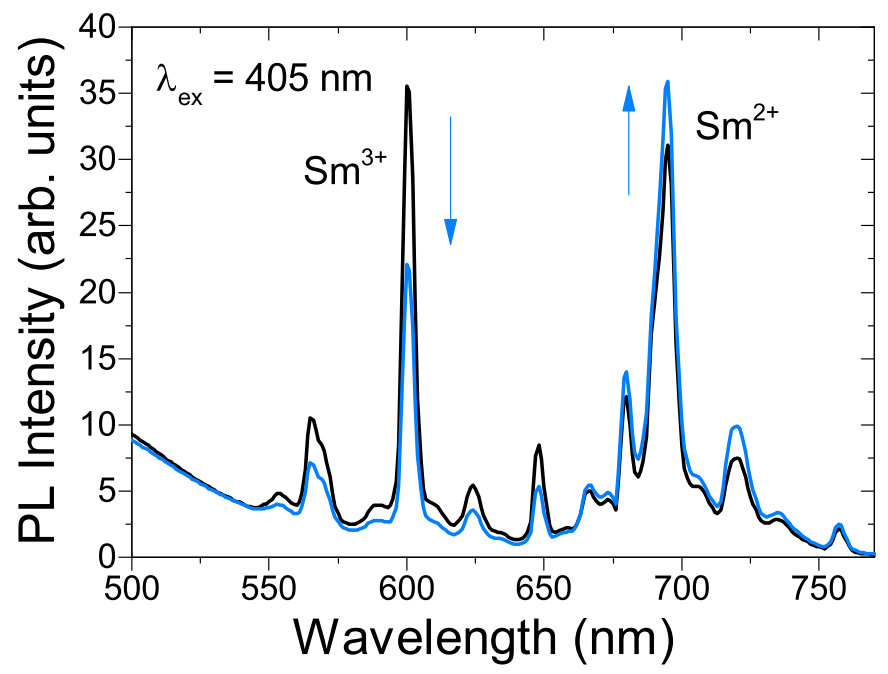

Figure 5.2: PL emission spectra of $\mathrm{NaMgF}_{3}: \mathrm{Sm}$ where $\lambda_{\text {ex }}=405 \mathrm{~nm}$ before (black) and after (blue) an X-ray dose of $100 \mathrm{~Gy}$.

Oxygen acts as a hole trap and the full reduction process may be written as follows:

$$
\mathrm{X} \text {-rays }+\mathrm{Sm}^{3+}+\mathrm{O}_{L}^{2-} \rightarrow \mathrm{Sm}^{3+}+\mathrm{O}_{L}^{2-}+\mathrm{e}^{-}+\mathrm{h}^{+} \rightarrow \mathrm{Sm}^{2+}+\mathrm{O}_{L}^{-},
$$

where $\mathrm{O}_{L}^{2-}$ is an oxygen ion localised to, though not necessarily adjacent to, the $\mathrm{Sm}^{3+}$ dopant.

The X-ray-induced conversion of $\mathrm{Sm}^{3+}$ to $\mathrm{Sm}^{2+}$ produced a detectable change in the PL properties that can be expressed as an increase in the ratio of PL intensities $I_{\mathrm{PL}}\left(\mathrm{Sm}^{2+}\right) / I_{\mathrm{PL}}\left(\mathrm{Sm}^{3+}\right)$. This RPL may be used to monitor radiation doses and will be explored in greater detail in Section 5.2. It was found that exposing an irradiated sample to $254 \mathrm{~nm}(4.9 \mathrm{eV})$ light for several hours partially restored the Sm valences to their initial configuration, suggesting that the $\mathrm{Sm}^{2+}$ level was at most $4.9 \mathrm{eV}$ below the conduction band of $\mathrm{NaMgF}_{3}$.

\subsection{2 $\mathrm{NaMgF}_{3}: \mathrm{Eu}$}

The PL emission spectra for $\mathrm{NaMgF}_{3}: \mathrm{Eu}$ are shown in figure 5.3 (a) for the excitations $\lambda_{\text {ex }}=340 \mathrm{~nm}$ and $\lambda_{\text {ex }}=395 \mathrm{~nm}$. When $\lambda_{\text {ex }}=340 \mathrm{~nm}$, sharp line emissions were observed at $569 \mathrm{~nm}$ and $625 \mathrm{~nm}$ that were attributed to the transitions from the ${ }^{5} \mathrm{D}_{0}$ excited state of $\mathrm{Eu}^{3+}$ to the ${ }^{7} \mathrm{~F}_{0}$ and ${ }^{7} \mathrm{~F}_{2}$ states, respectively. The broad emission component that increased below $\sim 450 \mathrm{~nm}$ was due to the $5 d \rightarrow 4 f$ emission of $\mathrm{Eu}^{2+}$ that has been previously characterised in detail $[27,28]$. When $\lambda_{\text {ex }}=395 \mathrm{~nm}$, similar line emissions were 

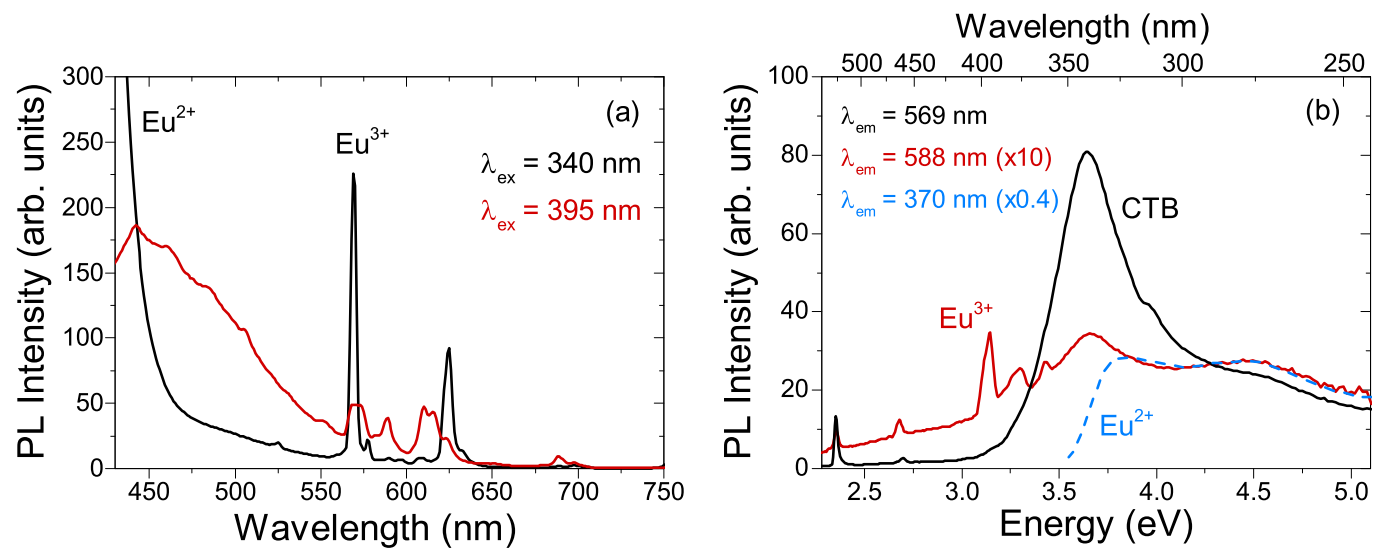

Figure 5.3: (a) PL emission spectra of $\mathrm{NaMgF}_{3}$ :Eu where $\lambda_{\text {ex }}=340 \mathrm{~nm}$ (black) and $\lambda_{\text {ex }}=$ $395 \mathrm{~nm}$ (red). (b) PL excitation spectra of $\mathrm{NaMgF}_{3}:$ Eu where $\lambda_{\mathrm{em}}=569 \mathrm{~nm}$ (black), $\lambda_{\mathrm{em}}$ $=588 \mathrm{~nm}(\times 10)(\mathrm{red})$, and $\lambda_{\mathrm{em}}=370 \mathrm{~nm}(\times 0.4)$ (blue dashed $)$.

observed that were also attributed to the various ${ }^{5} \mathrm{D}_{0} \rightarrow{ }^{7} \mathrm{~F}_{\mathcal{f}}$ transitions (table 5.2). The broad emission peaking near $450 \mathrm{~nm}$ was due to oxygen impurities.

The excitation spectra for the several distinct emissions are shown in figure $5.3(\mathrm{~b})$. When $\lambda_{\text {em }}=588 \mathrm{~nm}$, corresponding to the ${ }^{5} \mathrm{D}_{0} \rightarrow{ }^{7} \mathrm{~F}_{1}$ emission, line excitations corresponding to $4 f \rightarrow 4 f$ transitions were superimposed on some broad background components. The higher energy components were due to $\mathrm{Eu}^{2+}$ excitations, as illustrated by the excitation spectrum when $\lambda_{\mathrm{em}}=370 \mathrm{~nm}$ (corresponding to the peak $\mathrm{Eu}^{2+}$ emission [28]). The excitation component peaking near $340 \mathrm{~nm}$ was not due to $\mathrm{Eu}^{2+}$ and was attributed to an $\mathrm{O}^{2-}$ related CTB. When the excitation wavelength was shifted to focus on the ${ }^{5} \mathrm{D}_{0} \rightarrow{ }^{7} \mathrm{~F}_{0}$ emission $\left(\lambda_{\text {em }}=569 \mathrm{~nm}\right)$ the CTB was enhanced. Also of note was the enhancement of the ${ }^{7} \mathrm{~F}_{0} \rightarrow{ }^{5} \mathrm{D}_{1}$ excitation at $528 \mathrm{~nm}$.

Unlike the $\mathrm{Sm}^{3+} \mathrm{CT}$, exciting into the CTB for $\mathrm{NaMgF}_{3}$ :Eu produced $\mathrm{Eu}^{3+}$ emissions with energies and intensities perturbed from those produced upon excitation into the various $4 f^{6}$ levels. This behaviour was not expected and suggests that the local environments of those $\mathrm{Eu}^{3+}$ ions that could be excited via $\mathrm{CT}$, i.e. the $\mathrm{Eu}^{3+}-\mathrm{O}^{2-}$ pairs, were significantly distorted relative to the standard $\mathrm{Eu}^{3+}$ ions with $\mathrm{F}^{-}$ligands.

The ${ }^{5} \mathrm{D}_{0} \rightarrow{ }^{7} \mathrm{~F}_{1}$ transition of $\mathrm{Eu}^{3+}$ is a pure magnetic dipole transition and is therefore largely independent of the crystalline environment [27, 154]. In contrast, the ${ }^{5} \mathrm{D}_{0} \rightarrow{ }^{7} \mathrm{~F}_{0}$ and ${ }^{5} \mathrm{D}_{0} \rightarrow{ }^{7} \mathrm{~F}_{2}$ transitions are forced electric dipole transitions that are strongly affected by the local environment $[27,154]$. Furthermore, the ${ }^{5} \mathrm{D}_{0} \rightarrow{ }^{7} \mathrm{~F}_{0}$ is forbidden by the $\mathcal{f}$ selection rule. Despite these properties, the ${ }^{5} \mathrm{D}_{0} \rightarrow{ }^{7} \mathrm{~F}_{0}$ emission was observed clearly under both $340 \mathrm{~nm}$ and $395 \mathrm{~nm}$ excitations. For the latter excitation, the ${ }^{5} \mathrm{D}_{0} \rightarrow{ }^{7} \mathrm{~F}_{0}$ 


\begin{tabular}{cc}
\hline $\mathrm{Eu}^{3+}$ & $\lambda(\mathrm{nm}) /$ Energy $(\mathrm{eV})$ \\
\hline \hline${ }^{5} \mathrm{D}_{0} \rightarrow{ }^{7} \mathrm{~F}_{4}$ & $690 / 1.80$ \\
\hline${ }^{5} \mathrm{D}_{0} \rightarrow{ }^{7} \mathrm{~F}_{2}$ & $610(625) / 2.03(1.98)$ \\
\hline${ }^{5} \mathrm{D}_{0} \rightarrow{ }^{7} \mathrm{~F}_{1}$ & $589 / 2.11$ \\
\hline${ }^{5} \mathrm{D}_{0} \rightarrow{ }^{7} \mathrm{~F}_{0}$ & $573(569) / 2.16(2.18)$ \\
\hline
\end{tabular}

Table 5.2: Energies of the most intense transitions of $\mathrm{Eu}^{3+}$ in $\mathrm{NaMgF}_{3}: \mathrm{Eu}$. Values in brackets correspond to emissions from the $\mathrm{S} 2 \mathrm{Eu}^{3+}$ sites.

emission was split into two components despite the non-degeneracy of the electronic states involved. This immediately indicated the existence of at least two unique $\mathrm{Eu}^{3+}$ sites, where the ${ }^{5} \mathrm{D}_{0} \rightarrow{ }^{7} \mathrm{~F}_{0}$ emissions peaked at $2.16 \mathrm{eV}(\mathrm{S} 1)$ and $2.18 \mathrm{eV}(\mathrm{S} 2)$. The ${ }^{5} \mathrm{D}_{0} \rightarrow$ ${ }^{7} F_{0}$ is expected only for sites with $C_{n}, C_{n v}$, or $C_{s}$ symmetries [154], further highlighting the degree of distortion.

Under $\lambda_{\mathrm{ex}}=340 \mathrm{~nm}$ stimulation into the $\mathrm{CTB}$, emissions from the $\mathrm{S} 2 \mathrm{Eu}^{3+}$ dominated the emission spectrum. The forced electric dipole transitions $\left({ }^{5} \mathrm{D}_{0} \rightarrow{ }^{7} \mathrm{~F}_{0}\right.$ and $\left.{ }^{5} \mathrm{D}_{0} \rightarrow{ }^{7} \mathrm{~F}_{2}\right)$ were greatly enhanced relative to the $\mathrm{S} 1 \mathrm{Eu}^{3+}$ emissions, and the magnetic transition $\left({ }^{5} \mathrm{D}_{0}\right.$ $\rightarrow{ }^{7} \mathrm{~F}_{1}$ ) was very weak. Analysing the ratio of the ${ }^{5} \mathrm{D}_{0} \rightarrow{ }^{7} \mathrm{~F}_{0}$ and ${ }^{5} \mathrm{D}_{0} \rightarrow{ }^{7} \mathrm{~F}_{1}$ emissions is a means of studying $\mathrm{Eu}^{3+}$ spectra in crystalline compounds [154, 155]. For the $\mathrm{S} 2$ sites of $\mathrm{NaMgF}_{3}: \mathrm{Eu}$ the ${ }^{5} \mathrm{D}_{0} \rightarrow{ }^{7} \mathrm{~F}_{0}$ peak intensity was approximately 30 times greater than the peak intensity of the ${ }^{5} \mathrm{D}_{0} \rightarrow{ }^{7} \mathrm{~F}_{1}$ emission. Similar relative intensities have been observed in other compounds, including $\mathrm{Sr}_{5}\left(\mathrm{PO}_{4}\right)_{3} \mathrm{~F}$ :Eu and $\mathrm{BaFCl}$ :Eu, when $\mathrm{O}^{2-}$ ions substitute for $\mathrm{F}^{-}$ions adjacent to the $\mathrm{Eu}^{3+}[154,155]$. Thus, all evidence suggests the formation of $\mathrm{Eu}^{3+}-$ $\mathrm{O}^{2-}$ centres, where charge transfer can occur between the constituent ions to produce intense $\mathrm{Eu}^{3+}$ emissions.

The effects of X-ray irradiation on $\mathrm{NaMgF}_{3}: \mathrm{Eu}$ are largely limited to OSL via the divalent $\mathrm{Eu}^{2+}$ ion. This will be explored in greater detail in Chapter 6.

\subsection{3 $\mathrm{NaMgF}_{3}: \mathrm{Dy}$}

The PL spectrum of $\mathrm{NaMgF}_{3}: \mathrm{Dy}$ where $\lambda_{\text {ex }}=350 \mathrm{~nm}$ is shown in figure 5.4 (a), where line emissions between $650 \mathrm{~nm}$ to $1500 \mathrm{~nm}$ were observed. The $350 \mathrm{~nm}$ stimulation resulted in excitations from the ${ }^{6} \mathrm{H}_{15 / 2}$ ground state to the ${ }^{6} \mathrm{P}_{7 / 2}$ excited state. The energies of the various excited states relative to the ground state are given in table 5.3. The excitation spectrum where $\lambda_{\mathrm{em}}=751 \mathrm{~nm}$ is shown in figure $5.4(\mathrm{~b})$, where all line excitations were 

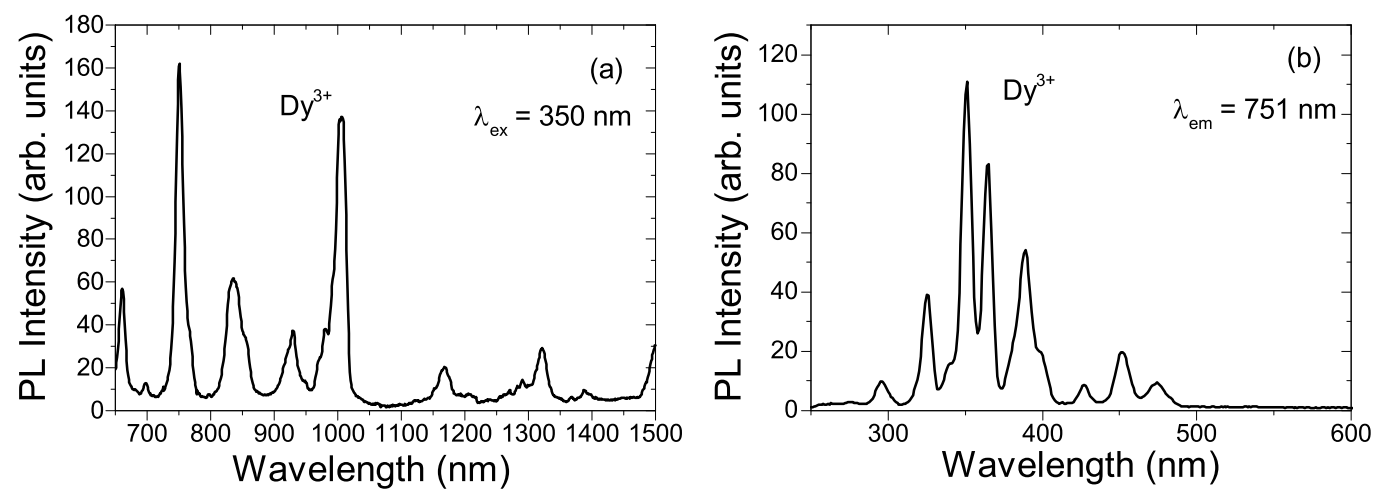

Figure 5.4: (a) PL emission spectra of $\mathrm{NaMgF}_{3}$ :Dy where $\lambda_{\mathrm{ex}}=350 \mathrm{~nm}$. (b) PL excitation spectra of $\mathrm{NaMgF}_{3}:$ Dy where $\lambda_{\mathrm{em}}=751 \mathrm{~nm}$.

attributed additional intraconfigurational $\mathrm{Dy}^{3+}$ transitions.

It should be noted that the numerous energy levels within the $4 f^{9}$ configuration of $\mathrm{Dy}^{3+}$ allow for multiple radiative transitions from a single excitation. This phenomenon is commonly referred to as downconversion, or quantum cutting, and has found applications in solar cell sensitisation [156]. In the $\mathrm{NaMgF}_{3}$ :Dy system several multi-photon emission pathways are available. For example, after excitation into the ${ }^{6} \mathrm{P}_{7 / 2}$ state (350 $\mathrm{nm})$ the $\mathrm{Dy}^{3+}$ ion may non-radiatively decay to the ${ }^{4} \mathrm{~F}_{9 / 2}$ state, then radiatively decay to the ${ }^{6} \mathrm{H}_{9 / 2}$ state and emit a photon $(751 \mathrm{~nm})$. Subsequently, the $\mathrm{Dy}^{3+}$ can radiatively decay to the ${ }^{6} \mathrm{H}_{15 / 2}$ state, and emit a second photon $(1332 \mathrm{~nm})$. While downconversion in is not explored further in this work, the result encourages future investigations of this and similar compounds for potential downconverter applications.

The $\mathrm{NaMgF}_{3}$ :Dy system differed from those compounds discussed previously ( $\mathrm{Ln}=$ $\mathrm{Sm}$ or $\mathrm{Eu}$ ) in that no divalent $\mathrm{Dy}^{2+}$ luminescence was observed prior to sample treatment. However, figure 5.5 illustrates that after exposure to X-ray irradiation (360 Gy) new luminescence features were observed and the features were characteristic of $\mathrm{Dy}^{2+}$. Few data exist in the literature on the $\mathrm{Dy}^{2+}$ ion due to its instability in most compounds [72]. As such, an analysis of the various energy levels of the $\mathrm{Dy}^{2+}$ ion was performed, drawing on data previously published for the isoelectronic $\mathrm{Ho}^{3+}$ ion.

When the sample was excited at $\lambda_{\mathrm{ex}}=350 \mathrm{~nm}$ prior to irradiation, only $\mathrm{Dy}^{3+}$ luminescence was seen (figure 5.5 (a)). After a $360 \mathrm{~Gy}$ X-ray dose, intense emissions between $1300 \mathrm{~nm}$ and $1500 \mathrm{~nm}$ were observed that were over 20 times more intense than the $\mathrm{Dy}^{3+}$ emissions. Emissions with similar line structure occurring between $1100 \mathrm{~nm}$ and $1200 \mathrm{~nm}$ have been observed in $\mathrm{NaYF}_{4}:$ Ho [156] and YAG:Ho [157], where they were attributed to the ${ }^{5} \mathrm{I}_{6} \rightarrow{ }^{5} \mathrm{I}_{8}$ transition of $\mathrm{Ho}^{3+}$. As $\mathrm{Ho}^{3+}$ and $\mathrm{Dy}^{2+}$ have the same $4 f^{10}$ 

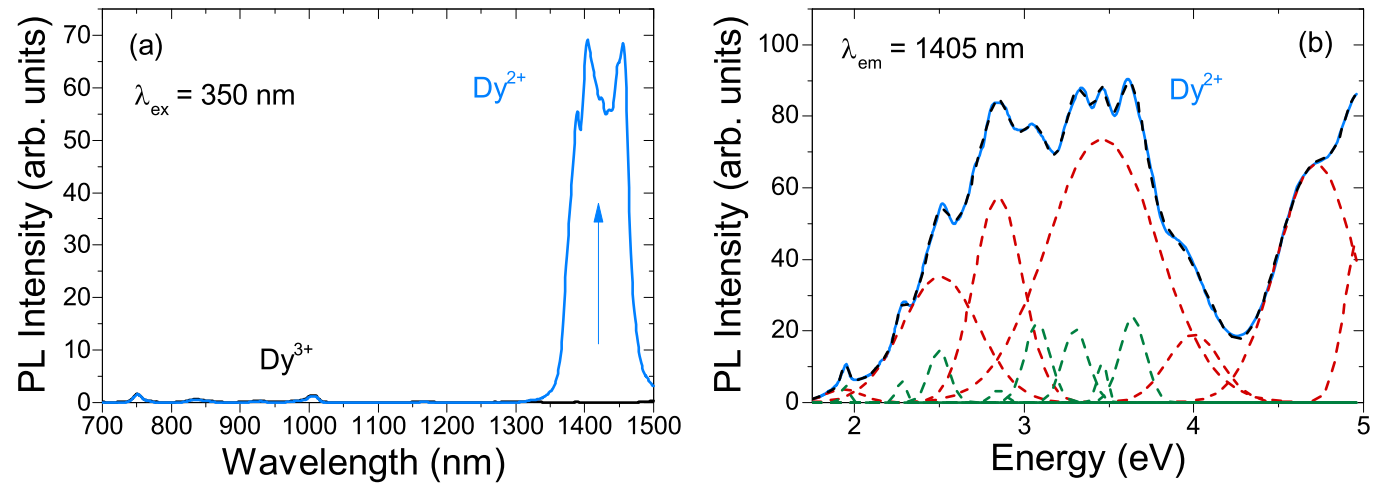

Figure 5.5: (a) PL emission spectra of $\mathrm{NaMgF}_{3}$ :Dy where $\lambda_{\mathrm{ex}}=350 \mathrm{~nm}$ before (black) and after (blue) an X-ray dose of $360 \mathrm{~Gy}$. (b) PL excitation spectra of $\mathrm{NaMgF}_{3}$ :Dy where $\lambda_{\mathrm{em}}$ $=1405 \mathrm{~nm}$ after an X-ray dose of 360 Gy. Dashed lines are Gaussian fits corresponding to $4 f \rightarrow 4 f$ transitions (green), $4 f \rightarrow 5 d$ transitions (red), and the cumulative fit (black).

configuration (and consequently the same ${ }^{2 S+1} \mathrm{~L}_{\mathrm{J}}$ states), a similar emission would be expected for the $\mathrm{Dy}^{2+}$ ion. Furthermore, the lower effective nuclear charge of $\mathrm{Dy}^{2+}$ relative to $\mathrm{Ho}^{3+}$ would result in smaller Coulomb and spin-orbit interactions and a reduction in the expected emission energy [72]. The $1430 \mathrm{~nm}$ emission observed in $\mathrm{NaMgF}_{3}$ :Dy after irradiation exhibits all behaviour expected for a $\mathrm{Dy}^{2+}$ ion and is attributed thus.

The PL excitation spectrum where $\lambda_{\mathrm{em}}=1405 \mathrm{~nm}$ is shown in figure $5.5(\mathrm{~b})$, where low-intensity $4 f \rightarrow 4 f$ excitations are superimposed on several stronger $4 f \rightarrow 5 d$ excitations. The few data available for the $\mathrm{Dy}^{2+}$ ion encouraged that the various $4 f \rightarrow 4 f$ transitions be investigated in greater detail. To that end, the data in figure 5.5 (b) were fitted to a sum of Gaussians corresponding to seven $4 f \rightarrow 5 d$ absorptions and ten $4 f \rightarrow$ If absorptions. Via comparisons with the $\mathrm{Ho}^{3+}$ ion in YAG:Ho and $\mathrm{NaYF}_{4}: \mathrm{Ho}[156,157]$, all transitions were attributed, and the energies of each transition are given in table 5.3.

The observation of $\mathrm{Dy}^{2+}$ luminescence post-irradiation indicated that the radiationinduced valence conversion $\mathrm{Dy}^{3+} \rightarrow \mathrm{Dy}^{2+}$ occurred. The RPL may be useful for monitoring radiation doses, where the main benefits of the compound are that the RPL signal prior to irradiation is zero (as no $\mathrm{Dy}^{2+}$ is observed), and that the emissions occur in the infrared spectral region. The latter property suggests that the compound could be used as a real-time RPL dosimeter, where the dose is monitored via the $\mathrm{Dy}^{2+}$ emission intensity, and where the $\mathrm{Dy}^{2+}$ emissions can be readily separated from any Cherenkov radiation component using long-pass optical filters.

In order to estimate the trap depth of the $\mathrm{Dy}^{2+}$ ion the $\mathrm{Dy}^{2+}$ emission intensity was 


\begin{tabular}{cccc}
\hline $\mathrm{Dy}^{3+}\left({ }^{6} \mathrm{H}_{15 / 2} \rightarrow\right)$ & $\lambda(\mathrm{nm}) / E(\mathrm{eV})$ & $\mathrm{Sm}^{2+}\left({ }^{5} \mathrm{I}_{8} \rightarrow\right)$ & $\lambda(\mathrm{nm}) / E(\mathrm{eV})$ \\
\hline${ }^{6} \mathrm{H}_{11 / 2}$ & $1797 / 0.69$ & ${ }^{5} \mathrm{I}_{6}$ & $1425 / 0.87$ \\
\hline${ }^{6} \mathrm{H}_{9 / 2}$ & $1363 / 0.91$ & ${ }^{5} \mathrm{~F}_{5}$ & $752 / 1.65$ \\
\hline${ }^{6} \mathrm{~F}_{9 / 2}$ & $1138 / 1.09$ & ${ }^{5} \mathrm{~F}_{4}$ & $636 / 1.95$ \\
\hline${ }^{6} \mathrm{H}_{5 / 2}$ & $1008 / 1.23$ & ${ }^{5} \mathrm{~F}_{3}$ & $574 / 2.16$ \\
\hline${ }^{6} \mathrm{~F}_{5 / 2}$ & $827 / 1.50$ & ${ }^{5} \mathrm{~F}_{2}$ & $544 / 2.28$ \\
\hline${ }^{4} \mathrm{~F}_{9 / 2}$ & $475 / 2.61$ & ${ }^{5} \mathrm{~F}_{1}$ & $496 / 2.50$ \\
\hline${ }^{4} \mathrm{I}_{15 / 2}$ & $453 / 2.74$ & ${ }^{3} \mathrm{G}_{5}$ & $435 / 2.85$ \\
\hline${ }^{4} \mathrm{G}_{11 / 2}$ & $428 / 2.9$ & ${ }^{5} \mathrm{G}_{4}$ & $403 / 3.08$ \\
\hline${ }^{4} \mathrm{I}_{13 / 2}$ & $389 / 3.19$ & ${ }^{5} \mathrm{G}_{5}$ & $376 / 3.30$ \\
\hline${ }^{4} \mathrm{I}_{11 / 2}$ & $365 / 3.4$ & ${ }^{5} \mathrm{G}_{3}$ & $358 / 3.46$ \\
\hline${ }^{6} \mathrm{P}_{7 / 2}$ & $351 / 3.53$ & ${ }^{5} \mathrm{G}_{2}$ & $341 / 3.64$ \\
\hline${ }^{6} \mathrm{P}_{3 / 2}$ & $325 / 3.82$ & $4 f^{9} 5 d^{1}$ & $642 / 1.93,494 / 2.51$, \\
& & & $435 / 2.85,358 / 3.46$, \\
& & & $248 / 4.99$ \\
\hline
\end{tabular}

Table 5.3: Energies of the $\mathrm{Dy}^{3+}$ and $\mathrm{Dy}^{2+}$ transitions in $\mathrm{NaMgF}_{3}: \mathrm{Dy}$. 
monitored over time during different stimulations. When excited into the lowest energy $5 d$ band $\left(\lambda_{\text {ex }}=635 \mathrm{~nm}\right)$ the emission intensity did not vary. When excited at higher energies $\left(\lambda_{\text {ex }}=340 \mathrm{~nm}\right)$ the $\mathrm{Dy}^{2+}$ emissions decreased by $70 \%$ over 1 hour and the decrease was permanent. This indicated that the trap depth of the $\mathrm{Dy}^{2+}$ was at maximum $3.65 \mathrm{eV}$.

\subsection{4 $\quad \mathrm{NaMgF}_{3}: \mathrm{Yb}$}
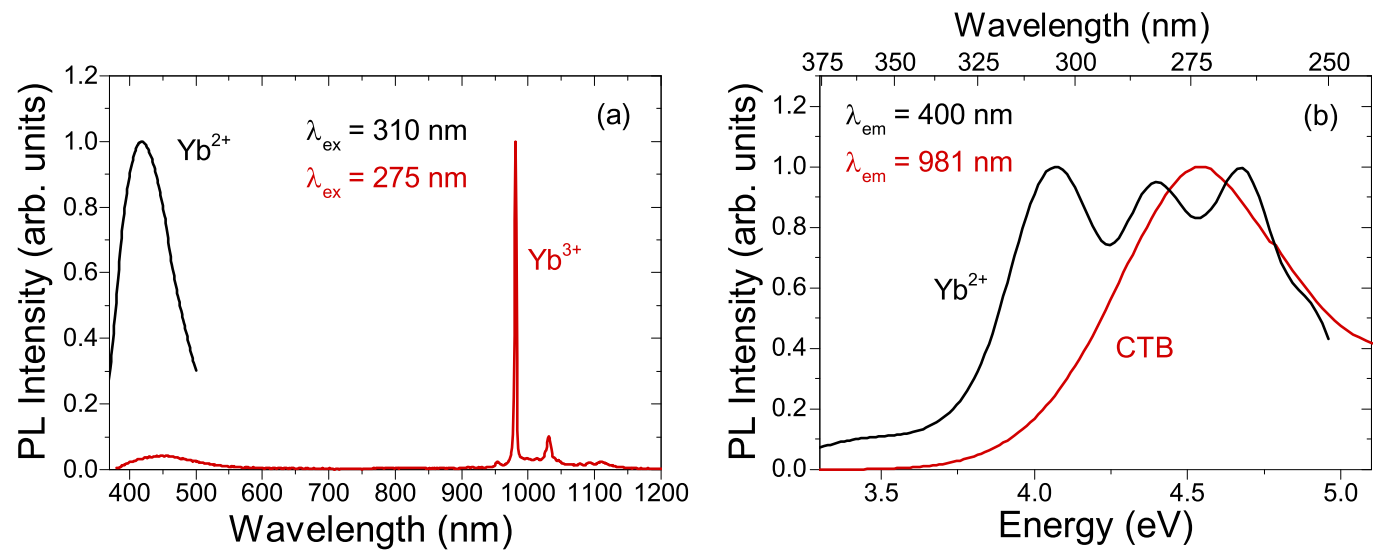

Figure 5.6: (a) Normalised PL emission spectra of $\mathrm{NaMgF}_{3}: \mathrm{Yb}$ where $\lambda_{\mathrm{ex}}=310 \mathrm{~nm}$ (black) and $\lambda_{\text {ex }}=275 \mathrm{~nm}$ (red). (b) Normalised PL excitation spectra of $\mathrm{NaMgF}_{3}: \mathrm{Yb}$ where $\lambda_{\mathrm{em}}$ $=400 \mathrm{~nm}$ (black) and $\lambda_{\mathrm{em}}=981 \mathrm{~nm}$ (red).

The normalised PL emission spectra of $\mathrm{NaMgF}_{3}: \mathrm{Yb}$ are shown in figure 5.6 (a). When excited at $\lambda_{\mathrm{ex}}=310 \mathrm{~nm}$, a broad emission peaking at $420 \mathrm{~nm}$ resulted that was due to $\mathrm{Yb}^{2+}$ transitions. When excited at $\lambda_{\text {ex }}=275 \mathrm{~nm}$, a broad emission centred about $450 \mathrm{~nm}$ was observed due to contaminant $\mathrm{O}^{2-}$ and sharp line emissions were observed in the IR between $950 \mathrm{~nm}$ and $1150 \mathrm{~nm}$ due to the split ${ }^{2} \mathrm{~F}_{5 / 2} \rightarrow{ }^{2} \mathrm{~F}_{7 / 2}$ transition of $\mathrm{Yb}^{3+}$. The presence of multiple line emissions indicated that there were multiple $\mathrm{Yb}^{3+}$ sites with different symmetries, likely due to several different charge-compensating neighbouring defects. The relevant excitation spectra are shown in figure 5.6 (b). When $\lambda_{\mathrm{em}}=400$ $\mathrm{nm}$ several split $4 f^{14} \rightarrow 4 f^{13} 5 d^{1}$ excitations were clear, occurring at $306 \mathrm{~nm}(4.05 \mathrm{eV})$, $281 \mathrm{~nm}(4.41 \mathrm{eV})$, and $265 \mathrm{~nm}(4.68 \mathrm{eV})$. The small contribution near $360 \mathrm{~nm}$ was due to the partial excitation of the $\mathrm{O}^{2-}$-related defect. The case of $\mathrm{Yb}^{3+}$ is distinct due to the existence of only one excited $4 f^{13}$ state (see figure 2.9 ), such that both excitation and emission could arise solely from the ${ }^{2} \mathrm{~F}_{5 / 2} \longleftrightarrow{ }^{2} \mathrm{~F}_{7 / 2}$ transition. However, $\lambda_{\mathrm{em}}=981 \mathrm{~nm}$ revealed a broad excitation band that peaked at $273 \mathrm{~nm}(4.54 \mathrm{eV})$ that gave rise to the $\mathrm{Yb}^{3+}$ luminescence. Per the previous arguments applied for the $\mathrm{NaMgF}_{3}: \mathrm{Sm}$ and $\mathrm{NaMgF}_{3}: \mathrm{Eu}$ 
CTBs, this excitation band was attributed to $\mathrm{O}^{2-} \rightarrow \mathrm{Yb}^{3+}$ charge transfer.

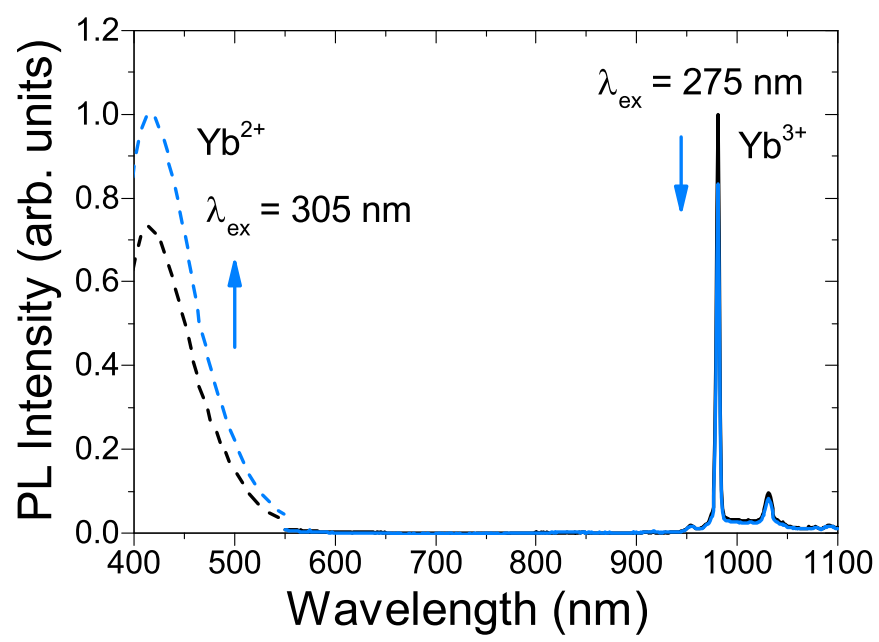

Figure 5.7: PL emission spectra of $\mathrm{NaMgF}_{3}: \mathrm{Yb}$ where $\lambda_{\mathrm{ex}}=305 \mathrm{~nm}$ (dashed lines) before (black) and after (blue) an X-ray dose of $90 \mathrm{~Gy}$, and where $\lambda_{\mathrm{ex}}=275 \mathrm{~nm}$ (solid lines) before (black) and after (blue) an X-ray dose of $90 \mathrm{~Gy}$.

The effect of X-ray exposure on the $\mathrm{Yb}$ luminescence in $\mathrm{NaMgF}_{3}: \mathrm{Yb}$ was examined and the data are presented in figure 5.7. Per the previously discussed samples, we predicted that $\mathrm{Yb}^{3+}$ would trap electrons during irradiation, forming additional $\mathrm{Yb}^{2+}$ ions and consequently producing RPL. An X-ray dose of 90 Gy confirmed this prediction, where the $\mathrm{Yb}^{2+}$ emission intensity increased post-irradiation while the $\mathrm{Yb}^{3+}$ emission intensity decreased. This RPL may be useful for radiation dose monitoring, where the $\mathrm{UV} /$ blue $\mathrm{Yb}^{2+}$ emissions are well-suited for PMT detection and could be monitored independently as a function of dose, or together with the IR emissions of $\mathrm{Yb}^{3+}$ that may be separated from any Cherenkov components.

Several recent studies have focused on the luminescence of $\mathrm{Yb}^{2+}$ in $\mathrm{NaMgF}_{3}: \mathrm{Yb}$ due to the exciton-like behaviour of the ion $[83,158,159]$. It was argued that the large Stokes shift was due to the emission arising from impurity trapped excitons (ITEs), where $\mathrm{Yb}^{2+}$ was the impurity, and the exciton was formed upon excitation into the $\mathrm{Yb}^{2+} 4 f^{13} 5 d^{1}$ states [158-160]. According to this model the excited $5 d$ electron is delocalised over nearby cations, effectively forming a short-lived $\left(\mathrm{Yb}^{3+}+\mathrm{e}^{-}\right)$centre before recombining and producing luminescence. In order to partially test this model the emission intensity of $\mathrm{Yb}^{2+}$ in irradiated $\mathrm{NaMgF}_{3}: \mathrm{Yb}$ was monitored as a function of time during continuous stimulation into the various $5 d$ bands. Up to the highest energy stimulation of approximately $5.1 \mathrm{eV}$, no variations were observed. This suggested that the optical trap depth of the $\mathrm{Yb}^{2+}$ was at least $5.1 \mathrm{eV}$, such that excitations into the $5 d$ band were insufficient 
to liberate electrons from the $\mathrm{Yb}^{2+}$ ion and into the conduction band. While this observation does not discount the ITE model, it does suggest that conduction band states are not involved, and that the electron of the $\left(\mathrm{Yb}^{3+}+\mathrm{e}^{-}\right)$pair is not sufficiently delocalised from the $\mathrm{Yb}$ ion so as to recombine with a trapped hole elsewhere.

\subsubsection{Establishing the Lanthanide Levels in $\mathrm{NaMgF}_{3}: \mathrm{Ln}$}

The data presented for the various $\mathrm{NaMgF}_{3}$ : $\mathrm{Ln}$ compounds allowed for a complete analyses of the $\mathrm{Ln}^{2+}$ and $\mathrm{Ln}^{3+}$ ions in the host. This analysis was made possible by the work of Dorenbos [67, 70,110,145, 148-150] during which systematic trends in the behaviour of the Ln ions in ionic compounds were established and a model with great predictive power was produced. While such analyses are typically performed using the energies of the $\mathrm{CT}$ transitions from the valence band to some $\mathrm{Ln}^{3+}$, e.g. $\mathrm{F}^{-} \rightarrow \mathrm{Eu}^{3+}$, in this work we have exploited the existence of $\mathrm{O}^{2-} \rightarrow \mathrm{Ln}^{3+}$ transitions in $\mathrm{NaMgF}_{3}$ as the energies of the $\mathrm{O}^{2-}$ CTBs were sufficiently low to detect using the spectrofluorometer employed in this work. This analysis was demonstrated to be possible in principle by Gerard [144], where $\mathrm{O}^{2-} \rightarrow \mathrm{Ln}^{3+}$ and $\mathrm{F}^{-} \rightarrow \mathrm{Ln}^{3+} \mathrm{CT}$ transitions were observed in the fluoride hosts $\mathrm{YF}_{3}: \mathrm{Ln}$ and $\mathrm{LaF}_{3}: \mathrm{Ln}$, and where the $\mathrm{O}^{2-}$ levels were determined to be approximately $2.6 \mathrm{eV}$ to $2.8 \mathrm{eV}$ above the valence bands. Here, the analysis was carried to completion, and a HRBE diagram for all $\mathrm{Ln}^{2+}$ and $\mathrm{Ln}^{3+}$ ions in $\mathrm{NaMgF}_{3}: \mathrm{Ln}$ was produced for the first time. HRBE diagrams provide a great deal of predictive power regarding the study of a compound as they place the ground state and first excited $5 d$ state energies for all $\mathrm{Ln}^{2+}$ and $\mathrm{Ln}^{3+}$ ions in the compound, relative to the valence and conduction bands of the host.

In the previous section, CTBs arising from $\mathrm{O}^{2-} \rightarrow \mathrm{Ln}^{3+}$ charge transfer were observed in the excitation spectra for $\mathrm{NaMgF}_{3}: \mathrm{Sm}, \mathrm{NaMgF}_{3}: \mathrm{Eu}$, and $\mathrm{NaMgF}_{3}: \mathrm{Yb}$. The most welldefined CTB was that of $\mathrm{NaMgF}_{3}: \mathrm{Yb}$ that peaked at $4.54 \mathrm{eV}$. Based on the aforementioned Dorenbos model [150] the ground state of $\mathrm{Yb}^{2+}$ should then be located $4.54 \mathrm{eV}$ above the $\mathrm{O}^{2-}$ level in $\mathrm{NaMgF}_{3}$. The $\mathrm{O}^{2-}$ level is then located some energy above the valence band. The first $5 d$ excitation of $\mathrm{Yb}^{2+}$ occurred $4.05 \mathrm{eV}$ above the ground state and is therefore located $8.59 \mathrm{eV}$ above the $\mathrm{O}^{2-}$ levels.

We then employed the Dorenbos model as follows. The energy of the $\mathrm{Ln}^{2+}$ ground state for any $\mathrm{Ln}^{2+}$ ion, $E_{\mathrm{Vf}}\left(\mathrm{Ln}^{2+}\right)$, relative to the $\mathrm{O}^{2-}$ band, may be calculated using the relation:

$$
E_{\mathrm{Vf}}\left(\operatorname{Ln}^{2+}\right)=E_{\mathrm{Vf}}\left(\mathrm{Yb}^{2+}\right)+\Delta E_{\mathrm{Vf}}\left(\operatorname{Ln}^{2+}\right)
$$


where $\Delta E_{\mathrm{Vf}}\left(\mathrm{Ln}^{2+}\right)$ is the energy difference between the ground state of $\mathrm{Ln}^{2+}$ and that of the reference ion, in this case $\mathrm{Yb}^{2+}$. The values of $\Delta E_{\mathrm{Vf}}\left(\operatorname{Ln}^{2+}\right)$ have been demonstrated to be approximately constant in different host compounds and are given for all $\mathrm{Ln}^{2+}$ in Ref. [67]. Thus, the ground state energies of all $\mathrm{Ln}^{2+}$ relative to the $\mathrm{O}^{2-}$ band were established.

The energies of the first $5 d$ levels relative to the ground state, $E_{\mathrm{fd}}\left(\operatorname{Ln}^{2+}\right)$, for all $\mathrm{Ln}^{2+}$ were also calculated, using the relation:

$$
E_{\mathrm{fd}}\left(\operatorname{Ln}^{2+}\right)=E_{\text {free }}\left(\operatorname{Ln}^{2+}\right)-D\left(\operatorname{Ln}^{2+}\right)
$$

where $E_{\text {free }}\left(\operatorname{Ln}^{2+}\right)$ are the known $E_{\mathrm{fd}}\left(\operatorname{Ln}^{2+}\right)$ values for the free $\operatorname{Ln}^{2+}$ ions $[67,148]$ and $D\left(\operatorname{Ln}^{2+}\right)$ is the red-shift parameter as defined by equation 5.2. As $D\left(\operatorname{Ln}^{2+}\right)$ is approximately constant for all $\mathrm{Ln}^{2+}$, we calculated $D\left(\mathrm{Ln}^{2+}\right)=D\left(\mathrm{Yb}^{2+}\right)=0.15 \mathrm{eV}$, and subsequently calculated all remaining $E_{\mathrm{fd}}\left(\mathrm{Ln}^{2+}\right)$ values via equation 5.2. Thus, the energies of the $5 d$ levels relative to the $4 f$ ground states were established for all $\mathrm{Ln}^{2+}$.

In order to establish the ground state energies of the $\mathrm{Ln}^{3+}$ ions, we used the centroid shift model as presented by Dorenbos [67]. The centroid shift, $\varepsilon_{\mathrm{c}}$, is defined as the difference between the average energy of the $4 f \rightarrow 5 d$ transitions for $\mathrm{Ce}^{3+}$ in a host compound and that of the free $\mathrm{Ce}^{3+}$ ion [67]. It was previously established that $\varepsilon_{\mathrm{c}}=0.594$ $\mathrm{eV}$ in $\mathrm{NaMgF}_{3}: \mathrm{Ce}$ [67]. An empirical relationship was established between the value of $\varepsilon_{\mathrm{c}}$ and the parameter $U(\mathrm{Eu})$, defined as the difference between the ground state energies of $\mathrm{Eu}^{3+}$ and $\mathrm{Eu}^{2+}$ in a compound, where $U(\mathrm{Eu})=7.60 \mathrm{eV}$ in $\mathrm{NaMgF}_{3}$. As $E_{\mathrm{Vf}}\left(\mathrm{Eu}^{2+}\right)$ was previously established with reference to the $\mathrm{O}^{2-}$ level, $E_{\mathrm{Vf}}\left(\mathrm{Eu}^{3+}\right)$ was calculated via $E_{\mathrm{Vf}}\left(\mathrm{Eu}^{3+}\right)=E_{\mathrm{Vf}}\left(\mathrm{Eu}^{2+}\right)-U(\mathrm{Eu})$, such that the ground state level of the $\mathrm{Eu}^{3+}$ ion was placed.

Similar to that process used to establish the $\mathrm{Ln}^{2+}$ levels other than $\mathrm{Yb}^{2+}$, the remaining $E_{\mathrm{Vf}}\left(\mathrm{Ln}^{3+}\right)$ levels could be calculated via:

$$
E_{\mathrm{Vf}}\left(\operatorname{Ln}^{3+}\right)=E_{\mathrm{Vf}}\left(\mathrm{Eu}^{3+}\right)+\Delta E_{\mathrm{Vf}}\left(\operatorname{Ln}^{3+}\right)
$$

where the $\Delta E_{\mathrm{Vf}}\left(\operatorname{Ln}^{3+}\right)$ values are approximately constant for different host materials are are given in [67]. Thus, the ground state energies for all $\mathrm{Ln}^{3+}$, relative to the $\mathrm{O}^{2-}$ level, were established.

The energies of the first $5 d$ levels for all $\mathrm{Ln}^{3+}$ could be similarly calculated. The first $5 d$ excitation for $\mathrm{Ce}^{3+}$ in $\mathrm{NaMgF}_{3}$ :Ce has been previously shown to occur at $245 \mathrm{~nm}$ (5.06 $\mathrm{eV})$, such that $E_{\mathrm{fd}}\left(\mathrm{Ce}^{3+}\right)$ is known [84]. Using equation 5.3, though where $E_{\mathrm{fd}}\left(\mathrm{Ln}^{3+}\right)$ and $\Delta E_{\mathrm{fd}}\left(\operatorname{Ln}^{3+}\right)$ are substituted for $E_{\mathrm{Vf}}\left(\operatorname{Ln}^{3+}\right)$ and $\Delta E_{\mathrm{Vf}}\left(\operatorname{Ln}^{3+}\right)$, the remaining $5 d$ levels were calculated for all $\mathrm{Ln}^{3+}$ in $\mathrm{NaMgF}_{3}: \operatorname{Ln}$. The values of $\Delta E_{\mathrm{fd}}\left(\operatorname{Ln}^{3+}\right)$ are given in [148]. 
Using the various methods given by Dorenbos, the energies of the ground state levels and first $5 d$ levels relative to the $\mathrm{O}^{2-}$ impurity band have been established for all $\mathrm{Ln}^{2+}$ and $\mathrm{Ln}^{3+}$ ions in $\mathrm{NaMgF}_{3}: \mathrm{Ln}$. The final challenge was then placing the $\mathrm{O}^{2-}$ level accurately within the band gap of the $\mathrm{NaMgF}_{3}$ host. We began by noting that previously published VUV excitation data for $\mathrm{NaMgF}_{3}$ suggested an approximate band gap energy of $12.4 \mathrm{eV}$ and a free exciton level $11.1 \mathrm{eV}$ above the valence band [83]. The energies of the $\mathrm{Ln}^{2+}$ ions, relative to the conduction band, could be approximated by the optical trap depths per the electron trapping observed via RPL. For $\mathrm{Sm}^{2+}, 254 \mathrm{~nm}(4.88 \mathrm{eV})$ light resulted in $\mathrm{Sm}^{2+} \rightarrow \mathrm{Sm}^{3+}$ post-irradiation. For $\mathrm{Dy}^{2+}, 340 \mathrm{~nm}(3.65 \mathrm{eV})$ light was sufficient to bleach the RPL. For $\mathrm{Yb}^{2+}, 5.1 \mathrm{eV}$ stimulation was insufficient to reverse the RPL. Using these data a range of possible $\mathrm{O}^{2-}$ levels were established, where the $\mathrm{O}^{2-}$ level was located between $2.16 \mathrm{eV}$ and $2.77 \mathrm{eV}$ above the valence band in $\mathrm{NaMgF}_{3}$. This range of values is in good agreement with the values obtained for $\mathrm{YF}_{3}: \mathrm{Ln}$ and $\mathrm{LaF}_{3}: \mathrm{Ln}$, as expected for the similar fluoride hosts [144].

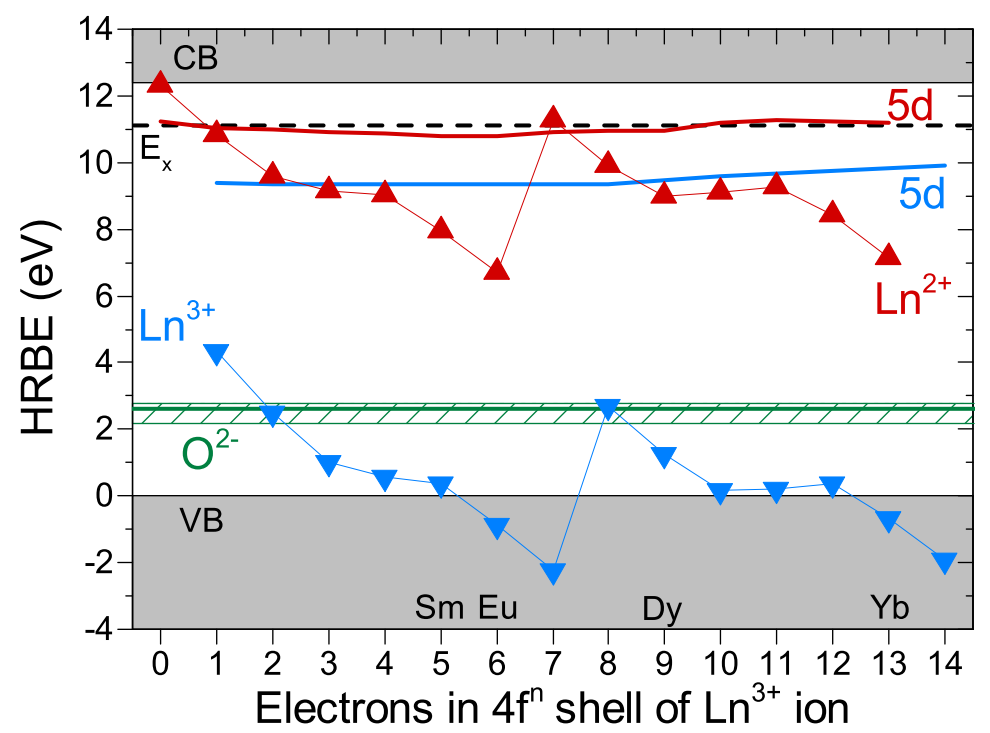

Figure 5.8: HRBE diagram for $\mathrm{NaMgF}_{3}: \mathrm{Ln}$. The $4 f^{\mathrm{n}}$ ground states for $\mathrm{Ln}^{2+}$ (red triangles) and $\mathrm{Ln}^{3+}$ (blue triangles) are shown, along with the first $5 d$ absorptions (red and blue lines, respectively). Also shown are the positions of the free exciton level (black dashed), the estimated $\mathrm{O}^{2-}$ impurity level (green dot-dashed), and the possible range of $\mathrm{O}^{2-}$ levels (green dashed area).

The HRBE diagram for $\mathrm{NaMgF}_{3}: \mathrm{Ln}$ is shown in figure 5.8, where the $\mathrm{O}^{2-}$ level was placed $2.6 \mathrm{eV}$ above the valence band, and where the valence band was equal to HRBE $=0 \mathrm{eV}$. Based on the uncertainty in this positioning, all values could be shifted by -0.44 
$\mathrm{eV}$ up to $+0.16 \mathrm{eV}$, as illustrated by the green shaded region, and remain consistent with the spectroscopic data. In any case, the diagram aids in predicting several properties of the $\mathrm{Ln}^{2+}$ and $\mathrm{Ln}^{3+}$ dopants in $\mathrm{NaMgF}_{3}: \mathrm{Ln}$, some of which will be discussed here.

The positioning of the $\mathrm{Ln}^{2+}$ levels in the band gap illustrates their potential to act as electron trapping sites with trap depths on the order of several eV. During X-ray stimulation significant concentrations of $\mathrm{Ln}^{3+}$ may be converted to $\mathrm{Ln}^{2+}$ upon electron capture, such that the converted ions occupy new positions within the band gap. It was shown earlier in this chapter that Sm, Dy, and $\mathrm{Yb}$ were converted from the trivalent to divalent states during irradiation, consistent with their positions approximately 4.44 $\mathrm{eV}, 3.42 \mathrm{eV}$, and $5.26 \mathrm{eV}$ below the conduction band, respectively. The large trap depths suggest that the converted ions are highly stable and therefore unlikely to be bleached near room temperature or without high energy optical stimulation. Furthermore, these values are entirely consistent with the optical bleaching of each converted ion - $\mathrm{Sm}^{2+}$ could be reverted to $\mathrm{Sm}^{3+}$ using $4.88 \mathrm{eV}$ light and $\mathrm{Dy}^{2+}$ could be reverted to $\mathrm{Dy}^{3+}$ using $3.65 \mathrm{eV}$ light, while $\mathrm{Yb}^{2+}$ could not be reverted to $\mathrm{Yb}^{3+}$ using $5.1 \mathrm{eV}$ light.

The diagram predicts the CT transition energies from both the $\mathrm{F}^{-}$and the $\mathrm{O}^{2-}$ bands. The lowest energy CT transition from the $\mathrm{F}^{-}$level was calculated to occur at $185 \mathrm{~nm}$ for $\mathrm{Eu}$ and therefore could not be detected using our equipment. The CT energies from the $\mathrm{O}^{2-}$ level were lowest in energy for $\mathrm{Eu}, \mathrm{Yb}$, and $\mathrm{Sm}$, at $302 \mathrm{~nm}, 273 \mathrm{~nm}$, and $231 \mathrm{~nm}$, respectively. These values are mostly consistent with the PL data, though the Eu CTB was shifted significantly from the expected value. This was likely due to the nature of the $\mathrm{Eu}^{3+}-\mathrm{O}^{2-}$ complex, where the sites were sufficiently distorted as to reduce the energy required to produce a $\mathrm{CT}$ transition. A study on $\mathrm{NaMgF}_{3}: \operatorname{Pr}$ measured the first $5 d$ level of the $\mathrm{Pr}^{3+}$ ion at $185 \mathrm{~nm}$, consistent with the value predicted by the HRBE diagram at $181 \mathrm{~nm}$.

Finally, the HRBE diagram predicts that a majority of the $\mathrm{Ln}^{3+}$ ions may trap electrons during irradiation, forming meta-stable $\mathrm{Ln}^{2+}$ ions and likely producing RPL phenomena. This was demonstrated for Eu, Sm, Dy, and $\mathrm{Yb}$, and is expected to occur for $\mathrm{Pr}, \mathrm{Nd}, \mathrm{Tb}$, Ho, Er, and Tm. Consequently, the investigation of alternative dopants is encouraged regarding RPL dosimeter applications using $\mathrm{NaMgF}_{3}$. The diagram further suggests that certain ions, particularly $\mathrm{Ce}, \mathrm{Pr}$, and $\mathrm{Tb}$ may function as stable hole traps that may be desirable in OSL applications, where the radiation-produced tetravalent ions may act as OSL recombination centres. 


\subsubsection{Summary and Discussion}

Section 5.1 focused on the basic PL properties of the $\mathrm{NaMgF}_{3}: \operatorname{Ln}$ compounds, where $\mathrm{Ln}$ $=\mathrm{Sm}, \mathrm{Eu}, \mathrm{Dy}$, or $\mathrm{Yb}$. Charge transfer transitions $\mathrm{O}^{2-} \rightarrow \mathrm{Ln}^{3+}$ were observed in the $\mathrm{PL}$ for $\mathrm{Ln}=\mathrm{Sm}, \mathrm{Eu}$, and $\mathrm{Yb}$. Excitation into the CTBs produced intense $\mathrm{Ln}^{3+}$ emissions in all cases, effectively circumventing the forbidden aspects of the intraconfigurational $4 f^{\mathrm{n}}$ transitions. Ultimately, this demonstrated that donor levels such as $\mathrm{O}^{2-}$ can be deliberately introduced into fluoride matrices to produce CTBs, and this effect may be exploited in the development of novel, strongly emitting phosphors [146, 161, 162]. Furthermore, the energies of the CTBs were used to establish the ground state energies of the divalent lanthanides relative to the oxygen levels and the techniques established by Dorenbos $[67,148,150]$ extended the analysis to include all $\mathrm{Ln}^{2+}$ ions.

RPL was observed for $\mathrm{Ln}=\mathrm{Sm}$, Dy, and $\mathrm{Yb}$, where radiation exposure resulted in the reduction of the trivalent $\mathrm{Ln}^{3+}$, described by equations of the form $\mathrm{Ln}^{3+}+\mathrm{e}^{-} \rightarrow$ $\mathrm{Ln}^{2+}$, where free conduction band electrons were produced during irradiation. The $\mathrm{Ln}^{3+}$ ions functioned as electron traps and the relative luminescence intensities of the $\mathrm{Ln}^{3+}$ and $\mathrm{Ln}^{2+}$ ions could be used to monitor cumulative radiation doses. For the $\mathrm{Ln}=\mathrm{Sm}$ sample, the most intense $\mathrm{Sm}^{3+}$ and $\mathrm{Sm}^{2+}$ emissions occurred between $550 \mathrm{~nm}$ and $750 \mathrm{~nm}$, such that optical filtering could be easily employed to distinguish between key emissions and the ratio of the two emissions monitored as a function of dose. For $\mathrm{Ln}=\mathrm{Yb}, \mathrm{Yb}^{2+}$ emissions occurred under UV stimulation and in the blue region of the optical spectrum, whereas $\mathrm{Yb}^{3+}$ emissions were also excited by UV stimulation though where emission occurred in the IR region. Consequently, each emitting centre was uniquely suited to a particular detection technique. IR emissions are desirable in the case of real-time fibre RPL dosimeters where the Cherenkov component could be easily filtered out. For Ln = Dy, no $\mathrm{Dy}^{2+}$ was observed prior to irradiation. Following irradiation, $\mathrm{Dy}^{2+}$ emissions were observed in the IR. The zero signal of $\mathrm{Dy}^{2+}$ for $0 \mathrm{~Gy}$ is useful in that the ratio of emissions need not necessarily be measured. As such, the most desirable compound for fibre RPL dosimetry was determined to be $\mathrm{NaMgF}_{3}$ :Dy. In any case, the observation of RPL in a majority of the samples studied is considered highly encouraging in the further development of $\mathrm{NaMgF}_{3}: \mathrm{Ln}$ compounds for dosimeter applications. It was demonstrated that the RPL could be optically reverted in the $\mathrm{Ln}=$ Sm and Dy samples, using $254 \mathrm{~nm}$ and $340 \mathrm{~nm}$ stimulation, respectively. At lower stimulation energies the RPL could be non-destructively probed in all cases.

The above information was used to establish the first HRBE diagram for the various $\mathrm{NaMgF}_{3}: \mathrm{Ln}$ compounds. As mentioned, the $\mathrm{O}^{2-} \rightarrow \mathrm{Ln}^{3+}$ CTBs were used to establish the 
$\mathrm{Ln}^{2+}$ ground state levels relative to the $\mathrm{O}^{2-}$ band. The work of Dorenbos $[67,148,150]$ allowed for placing the additional $\mathrm{Ln}^{2+}$ ions not studied in this work. A similar analysis extended to the various lowest energy $5 d$ levels for all $\mathrm{Ln}^{2+}$, where the $5 d$ excitations observed in the PL for $\mathrm{Sm}^{2+}, \mathrm{Dy}^{2+}$, and $\mathrm{Yb}^{2+}$ provided the initial references. Additional work by Dorenbos [67] demonstrated that the centroid shift of $\mathrm{Ce}^{3+}$ luminescence may be used to place the $\mathrm{Ln}^{3+}$ levels relative to the $\mathrm{Ln}^{2+}$ levels, and this technique was employed to establish the ground state energies of all $\mathrm{Ln}^{3+}$ ions relative to the $\mathrm{O}^{2-}$ band. The $\mathrm{Ce}^{3+} 5 d$ excited states in $\mathrm{NaMgF}_{3}: \mathrm{Ce}$ [84] were then used to place the $5 d$ levels for the remaining $\mathrm{Ln}^{3+}$ ions. Finally, the position of the $\mathrm{O}^{2-}$ level relative to the valence and conduction bands of $\mathrm{NaMgF}_{3}$ was estimated using the aforementioned RPL data, where certain stimulations were capable of reverting the X-ray induced valence conversions $\mathrm{Ln}^{3+} \rightarrow \mathrm{Ln}^{2+}$.

The HRBE diagram is shown in figure 5.8 and will provide a valuable reference in all future studies of the $\mathrm{NaMgF}_{3}: \mathrm{Ln}$ compounds. The diagram places the trap depths of those ions investigated in this work, namely $\mathrm{Sm}^{2+}, \mathrm{Dy}^{2+}$, and $\mathrm{Yb}^{2+}$, at $4.44 \mathrm{eV}, 3.42 \mathrm{eV}$, and $5.26 \mathrm{eV}$, respectively. The large trap depths indicate that the valence conversion is highly stable, further highlighting the potential for RPL dosimeter applications where signal fading would likely be negligible. Additionally, the diagram indicates that various other Ln ions, e.g. Ho, Er, and Pr, should exhibit RPL post-irradiation due to the positions of the $\mathrm{Ln}^{2+}$ levels relative to the conduction band.

Luminescence from both the $\mathrm{Ln}^{3+}$ and $\mathrm{Ln}^{2+}$ ions was observed for all studied samples, though only after irradiation in the case of $\mathrm{Dy}^{2+}$. The relative concentrations of each ion could not be reliably estimate from the PL data due to the nature of the various excitations, where the $4 f \rightarrow 5 d$ transitions for the divalent ions have oscillator strengths approximately $10^{4}$ times greater than those of the $4 f \rightarrow 4 f$ transitions of the trivalent ions. However, some comparisons can be made. We may note that the relative emission intensities, $\mathrm{Ln}^{3+} / \mathrm{Ln}^{2+}$, increased throughout the series as $\mathrm{Eu}, \mathrm{Yb}, \mathrm{Sm}$, Dy. This order correlated with the positions of the $\mathrm{Ln}^{2+}$ levels in the HRBE diagram, where the greater the $\mathrm{Ln}^{2+}$ trap depth the higher the apparent concentration of $\mathrm{Ln}^{2+}$. In keeping with this trend, we expect the alternative dopants $\mathrm{Ln}=\mathrm{Pr}, \mathrm{Nd}$, Ho, and $\mathrm{Er}$ to exhibit little to no $\mathrm{Ln}^{2+}$ luminescence prior to irradiation, a property that may aid in the development of additional RPL materials.

The observation of $\mathrm{Dy}^{2+}$ luminescence was also of interest from an experimental viewpoint, as $\mathrm{Dy}^{2+}$ is an uncommon ion due to its instability in most compounds. Consequently, a comprehensive analysis of the transition energies was performed, where 
the energies of the various $4 f \rightarrow 4 f$ and $4 f \rightarrow 5 d$ excitation bands were established via Gaussian fitting of the excitation spectrum. The particular states involved in each $4 f \rightarrow$ $4 f$ transition were evaluated in comparison to the emissions of the isoelectronic $\mathrm{Ho}^{3+}$ ion. Ultimately, a reference data set detailing the transitions of the $\mathrm{Dy}^{2+}$ ion and the energies of each excited state relative to the ${ }^{5} \mathrm{I}_{8}$ ground state was compiled (table 5.3) that should be of assistance in any future studies of the ion.

The behaviour of the $\mathrm{Eu}^{3+}$ ion was somewhat unique among the Ln series. While the $\mathrm{O}^{2-} \rightarrow \mathrm{Ln}^{3+}$ charge transfer energies were well-described by the HRBE diagram for $\mathrm{Ln}=\mathrm{Sm}$ and $\mathrm{Yb}$, the $\mathrm{O}^{2-} \rightarrow \mathrm{Eu}^{3+}$ was $0.45 \mathrm{eV}$ lower than expected. Additionally, at least two unique $\mathrm{Eu}^{3+}$ sites (S1 and S2) were observed in the compound via the distinct ${ }^{5} \mathrm{D}_{0} \rightarrow$ ${ }^{7} \mathrm{~F}_{0}$ line emissions. The $\mathrm{S} 2$ emissions that arose from excitation into the CTB behaved as would be expected for a highly distorted $\mathrm{Eu}^{3+}$ site, where the ratio of the ${ }^{5} \mathrm{D}_{0} \rightarrow{ }^{7} \mathrm{~F}_{0}$ emission to the ${ }^{5} \mathrm{D}_{0} \rightarrow{ }^{7} \mathrm{~F}_{1}$ emission was approximately 30 . These features were attributed to the formation of $\mathrm{Eu}^{3+}-\mathrm{O}^{2-}$ centres, as was necessary for the existence of CTBs, though where the centres were highly distorted relative to the typical Eu-F structures.

In summary, a framework was constructed that will be useful for any subsequent investigation of the $\mathrm{NaMgF}_{3}: \mathrm{Ln}$ compounds. All luminescence features discussed in the following section will reference the aforementioned framework where possible.

\subsection{Dosimetric Properties of $\mathrm{NaMgF}_{3}: \mathrm{Sm}$}

In the previous section it was demonstrated that several $\mathrm{NaMgF}_{3}: \mathrm{Ln}$ compounds may operate as RPL dosimeters where the X-ray induced valence conversion $\operatorname{Ln}^{3+} \rightarrow \operatorname{Ln}^{2+}$ was detectable via PL. It was then of interest to select a viable candidate and study in detail the optical and luminescence properties, and the effect of X-ray exposure on those properties. To that end, $\mathrm{NaMgF}_{3}: 0.05 \% \mathrm{Sm}$ was selected, and a study of its dosimeter properties is presented in this section.

$\mathrm{NaMgF}_{3}: \mathrm{Sm}$ has well-defined $\mathrm{Sm}^{3+}$ and $\mathrm{Sm}^{2+} 4 f \rightarrow 4 f$ emissions that occur between $550 \mathrm{~nm}$ and $650 \mathrm{~nm}$ for $\mathrm{Sm}^{3+}$ and $650 \mathrm{~nm}$ and $770 \mathrm{~nm}$ for $\mathrm{Sm}^{2+}$. Both valences could be stimulated with $405 \mathrm{~nm}$ light, corresponding to excitations from the ${ }^{6} \mathrm{H}_{5 / 2}$ ground state to the ${ }^{4} \mathrm{~F}_{7 / 2}$ excited in the case of $\mathrm{Sm}^{3+}$, and into a $4 f^{5} 5 d^{1}$ excited state for $\mathrm{Sm}^{2+}$. Analysis of the relative luminescence intensities for each valence was straightforward when monitored via spectrofluorometer and could also be reliably monitored using a photodetector coupled with suitable optical filtering.

Throughout this section, properties including optical absorption, magnetisation, OSL, 
$\mathrm{RPL}, \mathrm{RL}$, and RIC in $\mathrm{NaMgF}_{3}: \mathrm{Sm}$ will be discussed. It will be demonstrated that a range of optically-active defects were produced in the compound during irradiation that were also OSL active, and thus the OSL from $\mathrm{Sm}^{3+}$ could be coupled with the RPL for more versatile radiation dose measurements. Both the OSL and RPL intensities were characterised as a function of X-ray dose. The RL of the compound was shown to be strongly dose history dependent and a charge kinetics model was developed that describes the RL kinetics with high accuracy. An alternative method of determining the divalent lanthanide trap depths based on the thermal quenching of the $\mathrm{Sm}^{2+}$ PL and RPL will be presented. RIC data for the compound will be presented, discussed, and modeled. Ultimately, it will be demonstrated that the $\mathrm{NaMgF}_{3}: \mathrm{Sm}$ compound is an exceptional candidate for mixed RPL/OSL/RL/RIC dosimetry.

\subsubsection{Optical Absorption}

The radiation-induced absorptions in $\mathrm{NaMgF}_{3}: \mathrm{Sm}$, as measured after exposure to a high X-ray dose of 7.2 kGy, are shown in figure 5.9 (a) and spanned from below $2 \mathrm{eV}$ to above $5 \mathrm{eV}$. Gaussian fitting of the data revealed an excessive number of components when considering the number of defects expected to form, i.e. several F-type centres. The absorption peaked near $300 \mathrm{~nm}$, close to that peak expected to arise from F-centres [130]. The sample was then bleached with $300 \mathrm{~nm}$ light for 1 hour and the absorption measured again. The $300 \mathrm{~nm}$ absorption was reduced by the bleach and the remaining absorptions resembled the PL excitation spectrum of $\mathrm{Sm}^{2+}$ (see figure 5.1 (b)). The post-bleaching absorption was thus attributed to $\mathrm{Sm}^{2+}$ absorptions, where the population of converted $\mathrm{Sm}^{3+} \rightarrow \mathrm{Sm}^{2+}$ appeared as radiation-induced absorptions. A subsequent $254 \mathrm{~nm}$ bleach for 1 hour reduced the absorption intensity further due to reversal of the Sm valence conversion.

In order to better understand those absorptions that were bleached by the $300 \mathrm{~nm}$ stimulation, the absorption spectrum taken after the $300 \mathrm{~nm}$ bleach was subtracted from that obtained following the $7.2 \mathrm{kGy}$ irradiation, and the resulting spectrum is plotted in figure 5.9 (b). The spectrum could be fitted to a sum of four Gaussians, peaking at $2.53 \mathrm{eV}(491 \mathrm{~nm}), 3.01 \mathrm{eV}(412 \mathrm{~nm}), 4.16 \mathrm{eV}(298 \mathrm{~nm})$, and $5.59 \mathrm{eV}(222 \mathrm{~nm})$. All absorptions have been previously observed in samples of irradiated pure $\mathrm{NaMgF}_{3}[130,132]$. The absorptions at $298 \mathrm{~nm}, 412 \mathrm{~nm}$, and $491 \mathrm{~nm}$ were attributed to F-centres, $\mathrm{F}_{2}$-centres, and $\mathrm{F}_{3}$-centres, respectively [130]. The additional high energy absorption has been previously attributed to interstitial aggregates, e.g. $\mathrm{H}_{2}$-centres, that may function as hole traps [130]. We noted that the radiation-induced production of F-type centres in the 
$\mathrm{NaMgF}_{3}: \mathrm{Sm}$ compound resembled that of the $\mathrm{NaMgF}_{3}: \mathrm{Mn}$ compound (Section 4.3.1), and thus it was likely that the optically active F-centres contributed to some OSL process in the Sm-doped compound.
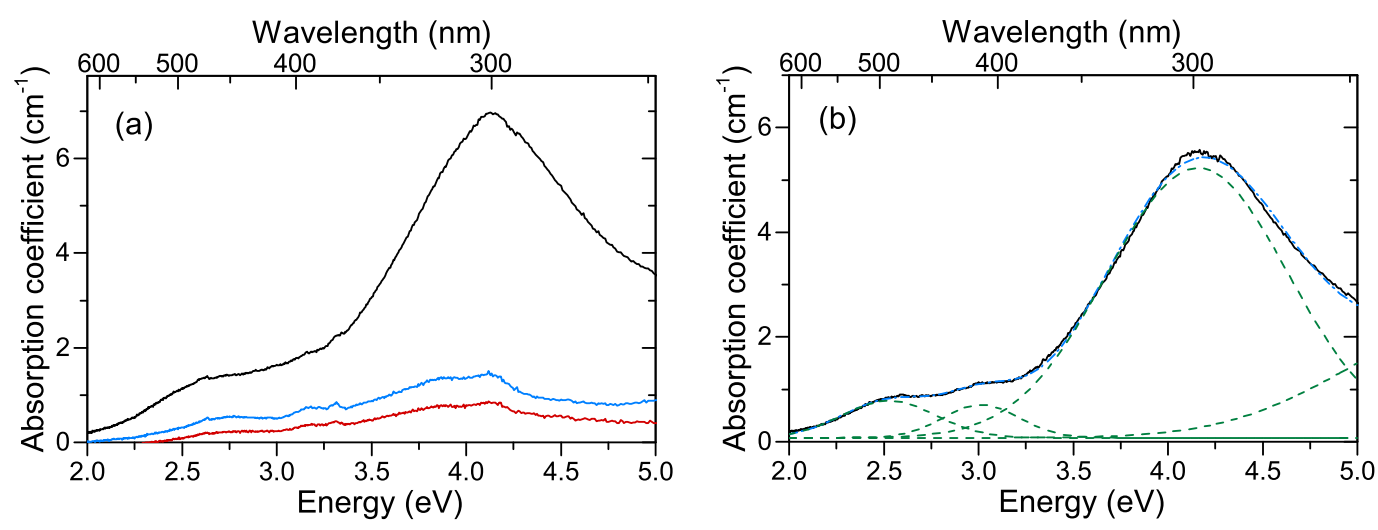

Figure 5.9: (a) Radiation-induced absorption coefficient of $\mathrm{NaMgF}_{3}: \mathrm{Sm}$ after a $7.2 \mathrm{kGy}$ $\mathrm{X}$-ray dose (black line), after $300 \mathrm{~nm}$ bleaching (blue line), and after $254 \mathrm{~nm}$ bleaching (red line). (b) Absorption coefficient after a $7.2 \mathrm{kGy}$ X-ray dose and after subtracting the absorptions that persisted after the $300 \mathrm{~nm}$ bleach. Individual Gaussian fits to the data are also shown (green dashed) along with the cumulative fit (blue dot-dashed).

Using Smakula's equation (equation 2.7) and the Gaussian corresponding to the Fcentre peak at $298 \mathrm{~nm}$, where the full-width at half-maximum was $0.96 \mathrm{eV}$ and the peak absorption was $5.15 \mathrm{~cm}^{-1}$, the concentration of $\mathrm{F}$-centres produced by the X-ray irradiation was calculated to be approximately $N_{\mathrm{F}} \approx 5 \times 10^{17} \mathrm{~cm}^{-3}$. Using equation 4.2 and the nominal total Sm concentration of $0.05 \%$ the approximate concentration of Sm ions was calculated to be $N_{\mathrm{Sm}} \approx 9 \times 10^{18} \mathrm{~cm}^{-3}$, over an order of magnitude greater than $N_{\text {F. }}$ All fluorine vacancies required charge compensation via the introduction of additional negative charge. Similar charge compensation was required for the substitution of $\mathrm{Sm}^{2+}$ and $\mathrm{Sm}^{3+}$ ions for the $\mathrm{Na}^{+}$ions of the $\mathrm{NaMgF}_{3}$ lattice. The compensation could be achieved through the introduction of $\mathrm{O}^{2-}$ ions, $\mathrm{F}^{-}$interstitials, and $\mathrm{Na}^{+}$vacancies, all of which could act as hole trapping sites. Consequently, the concentrations of the various additional defects should be greater than the concentration of F-centres.

\subsubsection{Magnetisation}

It was of interest to determine the relative concentrations of $\mathrm{Sm}^{3+}$ and $\mathrm{Sm}^{2+}$ in the compound, both after the initial synthesis and after an X-ray irradiation. This was achieved through measurements of the magnetic susceptibility of the compound, where the sus- 


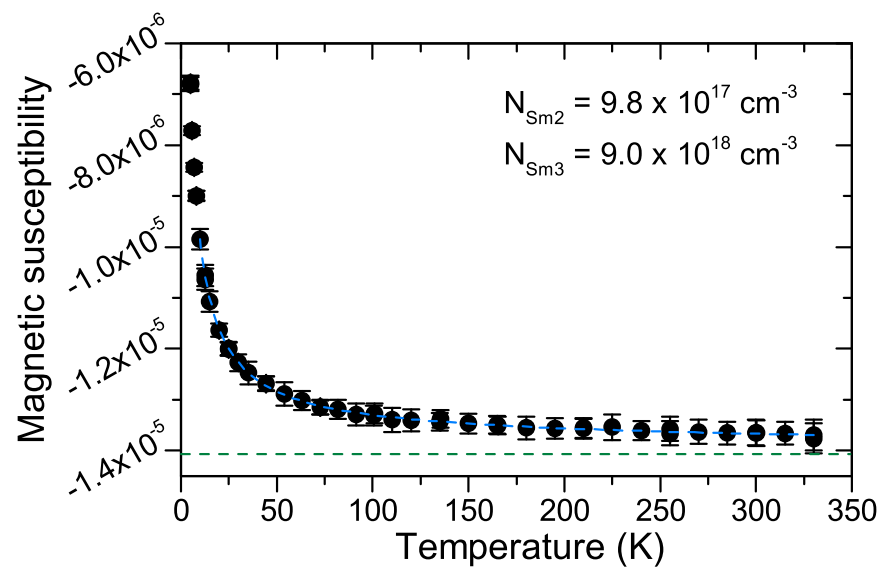

Figure 5.10: Magnetic susceptibility of $\mathrm{NaMgF}_{3}: \mathrm{Sm}$ at a magnetic field of $1 \mathrm{~T}$ (black circles). Also shown is the fit using equation 5.4 (blue dashed), and the diamagnetic background contribution due to the host lattice (green dashed).

ceptibility for temperatures $5 \mathrm{~K}$ to $330 \mathrm{~K}$ and at a field of $1 \mathrm{~T}$ is shown for an as-made sample of $\mathrm{NaMgF}_{3}: 0.05 \% \mathrm{Sm}$ in figure 5.10. The $\mathrm{Sm}^{3+}$ and $\mathrm{Sm}^{2+}$ ions exhibited paramagnetic behaviour on account of their unpaired $4 f$ electrons [163, 164], leading to increased susceptibilities at lower temperatures. The $\mathrm{NaMgF}_{3}$ host exhibited diamagnetic behaviour that manifested as a temperature-independent background. Furthermore, we accounted for a small paramagnetic component arising from other Ln impurities found in low concentrations, primarily contaminant $\mathrm{Eu}^{2+}$, the presence of which was verified by the presence of a weak line emission at $360 \mathrm{~nm}$ in the PL for a sample of $\mathrm{NaMgF}_{3}: \mathrm{Sm}$. Ultimately, the magnetic susceptibility, $\chi_{\mathrm{T}}$, could be described as the sum of multiple components written

$$
\chi_{\mathrm{T}}=\chi_{0}+\chi_{\mathrm{C}}+\chi_{2+}+\chi_{3+},
$$

where $\chi_{0}$ was the diamagnetic contribution from the $\mathrm{NaMgF}_{3}$ lattice, $\chi_{\mathrm{C}}$ was the paramagnetic susceptibility of the contaminant $\mathrm{Eu}^{2+}$, and $\chi_{2+}$ and $\chi_{3+}$ were the paramagnetic susceptibilities of the $\mathrm{Sm}^{2+}$ and $\mathrm{Sm}^{3+}$ ions, respectively.

The diamagnetic susceptibility of the host could be estimated as the sum of the susceptibilities of the $\mathrm{Na}^{+}, \mathrm{Mg}^{2+}$, and $\mathrm{F}^{-}$ions that constituted the host lattice. The susceptibilities of the individual ions are well known [165], such that $\chi_{0} \approx 1.43 \times 10^{-5}$. The calculations were significantly more complicated in the case of $\mathrm{Sm}^{2+}$ and $\mathrm{Sm}^{3+}$, as both ions were expected to exhibit Van Vleck paramagnetism [163, 164]. For this work, the $\mathrm{Sm}^{2+}$ states considered to contribute meaningfully to the paramagnetism were the ${ }^{7} \mathrm{~F}_{0}$, 
${ }^{7} \mathrm{~F}_{1}$, and ${ }^{7} \mathrm{~F}_{2}$ states. In the case of $\mathrm{Sm}^{3+}$, the ${ }^{6} \mathrm{H}_{5 / 2}$ and ${ }^{6} \mathrm{H}_{7 / 2}$ states were considered. The Van Vleck contributions to the susceptibility may be expressed as

$$
\chi_{\mathrm{vV}}=\frac{\mu_{\mathrm{P}} N_{i} \sum_{J}\left(A_{J} / T+\delta_{J}\right) B_{J} \exp \left(-D_{J} / T\right)}{\sum_{J} B_{J} \exp \left(-D_{J} / T\right)}
$$

where $\mu_{\mathrm{P}}=\mu_{0} \mu_{\mathrm{B}}, N_{i}$ is the concentration of the paramagnetic ion $i$, and $A_{J}=\left(g_{J}^{2} J(J+\right.$ $1) / 3 k_{\mathrm{B}}, B_{J}=2 J+1, D_{J}=E_{J} / k_{\mathrm{B}}$, where $\mu_{0}$ is the permeability of free space, $\mu_{\mathrm{B}}$ is the Bohr magneton, $g_{J}$ is the Lande g-factor, $J$ is the total angular momentum, $k_{\mathrm{B}}$ is the Boltzmann constant, and $E_{J}$ is the energy of the excited state with angular momentum number $J$ relative to the ground state. The $E_{J}$ values were determined via analysis of the PL spectra in figure 5.1. Furthermore, the variable $\delta_{J}$ is calculated via

$$
\delta_{J}=\frac{1}{6 B_{J}}\left(\frac{F(J+1)}{E_{J+1}-E_{J}}+\frac{F(J)}{E_{J}-E_{J-1}}\right)
$$

where

$$
F(J)=\frac{1}{J}\left[(S+L+1)^{2}-J^{2}\right]\left[J^{2}-(S-L)^{2}\right],
$$

and where $S$ and $L$ are the spin angular momenta and orbital angular momenta, respectively.

The contaminant $\mathrm{Eu}^{2+}$ contributions could be described via the Curie-Weiss law, written

$$
X_{\mathrm{C}}=\frac{\mu_{\mathrm{P}} N_{i} g_{J}^{2} J(J+1)}{3 k_{\mathrm{B}}\left(T-T_{\mathrm{C}}\right)},
$$

where $T_{\mathrm{C}}$ is the Curie-Weiss temperature.

Ultimately, equations 5.5 - 5.8 were substituted into equation 5.4 which was then fitted to the susceptibility data of figure 5.10. The fitted parameters were $\chi_{0}=1.4 \times 10^{-5}$, $N_{\mathrm{Eu}}=2.1 \times 10^{17} \mathrm{~cm}^{-3}, N_{2+}=9.8 \times 10^{17} \mathrm{~cm}^{-3}$, and $N_{3+}=9 \times 10^{18} \mathrm{~cm}^{-3}$, with uncertainties in the latter three parameters on the order of $10 \%$. Based on these values, we determined that approximately $10 \%$ of the total $\mathrm{Sm}$ concentration substituted into $\mathrm{NaMgF}_{3}$ in the divalent state, while the remaining $90 \%$ occupied the trivalent state. The diamagnetic background was in close agreement with the calculated value of $1.43 \times 10^{-5}$. The concentration of $\mathrm{Eu}^{2+}$ corresponded to a dopant fraction of approximately $0.001 \%$. In order to justify this concentration, the magnetic susceptibility of a nominally pure sample was studied and the data were fitted to a single Curie-Weiss component. The fitting yielded a similar $\mathrm{Eu}^{2+}$ concentration to that of the $\mathrm{NaMgF}_{3}: \mathrm{Sm}$ sample, confirming that the ad- 
ditional paramagnetic component was due to contaminants.

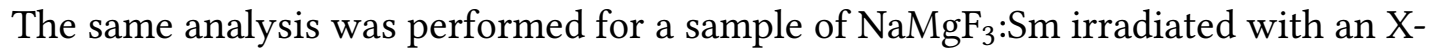
ray dose of $7.2 \mathrm{kGy}$, then stimulated with $290 \mathrm{~nm}$ light to bleach the radiation-induced F-centres. The calculated parameters $N_{2+}$ and $N_{3+}$ were within the uncertainties of those values calculated for the non-irradiated sample, suggesting that the total concentration of $\mathrm{Sm}^{3+}$ converted to $\mathrm{Sm}^{2+}$ did not exceed approximately $10 \%$ of the initial concentration.

\subsubsection{Optically Stimulated Luminescence}

The observation of optically stimulable radiation-induced defects in the absorption (figure 5.9) suggested that OSL should occur in $\mathrm{NaMgF}_{3}: \mathrm{Sm}$, provided a radiative recombination site existed. This was found to be the case, where $\mathrm{Sm}^{3+}$ acted as the recombination centre for the OSL. Figure 5.11 (a) shows the OSL stimulation curve after an X-ray dose of $2400 \mathrm{~Gy}$, obtained by subtracting the excitation spectrum where $\lambda_{\mathrm{em}}=600 \mathrm{~nm}$ prior to the irradiation from that taken after the irradiation. The primary OSL peak occurred at $4.4 \mathrm{eV}(281 \mathrm{~nm})$ and thus correlated with the F-centre peak seen in the absorption. An additional contribution to the OSL spectrum peaked at $3.0 \mathrm{eV}(413 \mathrm{~nm})$ that correlated with the $\mathrm{F}_{2}$-centre absorption. However, the appearance of an $\mathrm{F}_{2}$-centre $\mathrm{PL}$ emission peaking at approximately $530 \mathrm{~nm}$ suggested that the presence of an $\mathrm{F}_{2}$-centre peak in the OSL stimulation spectrum may instead have resulted purely from excitation into the $\mathrm{F}_{2}$-band. Any contribution to the OSL from the higher energy absorption at $5.59 \mathrm{eV}$ could not be resolved using the PL due to overlap with the $\mathrm{Sm}^{3+}$ CTB.

No OSL was observed from $\mathrm{Sm}^{2+}$ ions, suggesting that the trapped holes required for recombination were largely localised to the $\mathrm{Sm}^{3+}$ ions. This was consistent with the relative valences of the substitutions $\left(\mathrm{Sm}^{3+}\right.$ for $\mathrm{Na}^{+} \mathrm{vs} . \mathrm{Sm}^{2+}$ for $\left.\mathrm{Na}^{+}\right)$, where the required charge compensation was more likely to produce hole-trapping defects in the vicinity of $\mathrm{Sm}^{3+}$. Consequently, the OSL process likely involved the excitation of electrons from F-centres to the conduction band, recombination of those excited electrons with holes trapped nearby $\mathrm{Sm}^{3+}$, and energy transfer to the nearby $\mathrm{Sm}^{3+}$ ions.

The OSL decay curves where $\lambda_{\mathrm{ex}}=295 \mathrm{~nm}$ and $\lambda_{\mathrm{em}}=600 \mathrm{~nm}$ as measured after exposing a sample of $\mathrm{NaMgF}_{3}: \mathrm{Sm}$ to X-ray doses ranging from $1 \mathrm{~Gy}$ to $360 \mathrm{~Gy}$ are shown in figure 5.11 (b), where the OSL intensity increased as a function of dose. The inset demonstrates that the OSL emission occurred as $\mathrm{Sm}^{3+}$ luminescence, where the PL signal prior to irradiation was subtracted from that after irradiation. Similar to the $\mathrm{NaMgF}_{3}: \mathrm{Mn}$ samples, the OSL decays could be fitted to a three-exponential decay per equation 4.4, where the decay constants $\tau_{\mathrm{i}}$ were constant for all doses and were $\tau_{1}=15.3 \mathrm{~s}, \tau_{2}=83.2$ 

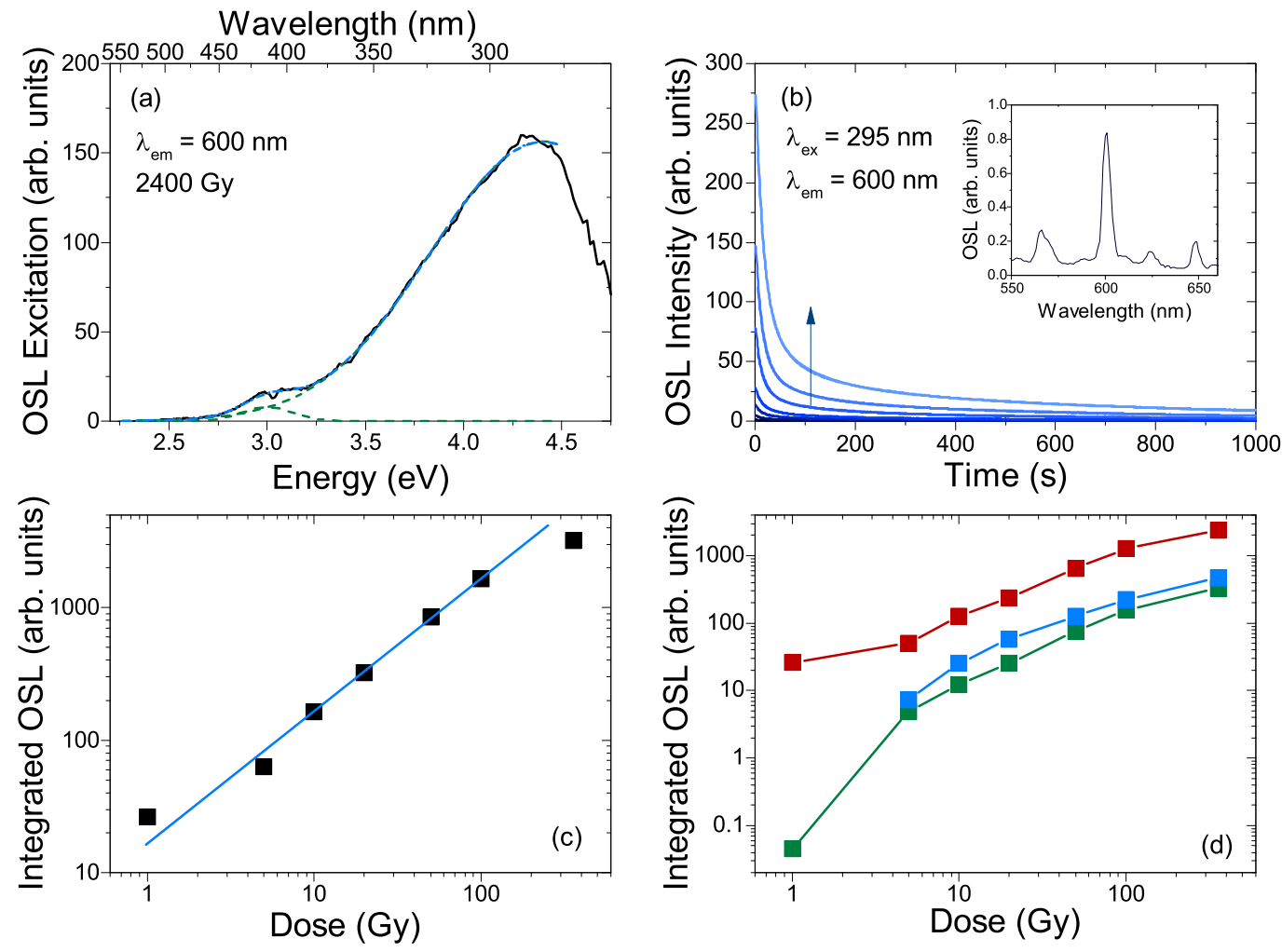

Figure 5.11: (a) OSL stimulation curve of $\mathrm{NaMgF}_{3}: \mathrm{Sm}$ after an X-ray dose of $2400 \mathrm{~Gy}$ and where $\lambda_{\mathrm{em}}=600 \mathrm{~nm}$. Individual Gaussian fits to the data are shown (green dashed), along with the cumulative fit (blue dot-dashed). (b) OSL decay curves where $\lambda_{\text {ex }}=295$ $\mathrm{nm}$ and $\lambda_{\mathrm{em}}=600 \mathrm{~nm}$ for various X-ray doses ranging from $1 \mathrm{~Gy}$ to $360 \mathrm{~Gy}$, where the arrow indicates the trend for increasing doses. Inset: OSL emission spectrum obtained after an X-ray dose of 2400 Gy. (c) Dose dependence of the integrated OSL emission intensity as determined by fitting the decay curves. The blue line is a linear fit to the data. (d) Integrated intensities of the individual exponential components, $A_{\mathrm{OSL}, 1}$ (green), $A_{\mathrm{OSL}, 2}$ (blue), and $A_{\mathrm{OSL}, 3}(\mathrm{red})$, as a function of X-ray dose. 
$\mathrm{s}$, and $\tau_{3}=750 \mathrm{~s}$.

The three exponential components required to fit the OSL decays were not initially expected as only two unique Gaussian components were observed in the stimulation spectrum. However, the optical absorption consisted of at least four components that were all bleached by continuous $300 \mathrm{~nm}$ stimulation. Consequently, the various exponential decay components were likely to have arisen from the several F-type centre configurations, where F-centre light $(\sim 300 \mathrm{~nm})$ was sufficiently energetic to bleach all centres, though where each centre had unique absorption cross-sections that produced different OSL decay lifetimes. The three exponential components were thus attributed to F-centres, $\mathrm{F}_{2}$-centres, and $\mathrm{F}_{3}$-centres with OSL decay constants $\tau_{1}, \tau_{2}$, and $\tau_{3}$, respectively.

The integrated OSL emission intensities, $A_{\mathrm{OSL}}(D)$ are plotted as a function of Xray dose in figure 5.11 (c), as calculated from the exponential fits where $A_{\mathrm{OSL}}=\sum_{i} A_{i} \tau_{i}$. $A_{\mathrm{OSL}}(D)$ was linear up to at least $100 \mathrm{~Gy}$, where the value at $360 \mathrm{~Gy}$ departed from linearity. The departure suggested that for high doses the concentrations of the OSLactive defects tended toward the saturation values as limited by the concentration of intrinsic trapping defects. This is illustrated in figure 5.11 (d), where the individual OSL components $A_{\mathrm{OSL}, \mathrm{i}}=A_{i} \tau_{i}$ are plotted as a function of dose and independently approach saturation values as the X-ray dose was increased.

The minimum detectable dose using the PL spectrometer, defined as the dose for which $A_{\mathrm{OSL}}(D)$ is equivalent to three standard deviations of the background signal, was calculated to be $1.2 \mathrm{mGy}$. This range of doses for which the OSL was linear indicates that the OSL $\mathrm{NaMgF}_{3}: \mathrm{Sm}$ is suitable for the detection of the radiation doses encountered in a radiotherapy context.

\subsubsection{Radiophotoluminescence}

The RPL of $\mathrm{NaMgF}_{3}: \mathrm{Sm}$ that manifested during X-ray irradiation as $\mathrm{Sm}^{3+} \rightarrow \mathrm{Sm}^{2+}$ valence conversion could also be used to monitor radiation doses, as illustrated in figure 5.12 (a). The PL spectrum where $\lambda_{\mathrm{ex}}=405 \mathrm{~nm}$ was measured for a sample prior to irradiation and following several subsequent irradiations. As the $405 \mathrm{~nm}$ stimulation did not revert any of the converted Sm populations to their original valences, the cumulative X-ray dose was the measurement of interest. Cumulative doses up to $1266 \mathrm{~Gy}$ were studied. The $\mathrm{Sm}^{2+}$ emissions above $650 \mathrm{~nm}$ increased with total dose, whereas the $\mathrm{Sm}^{3+}$ emissions below $650 \mathrm{~nm}$ decreased with dose. It was suggested in Section 5.1.1 that the ratio of the emissions from each Sm valence could be used to monitor radiation doses, 

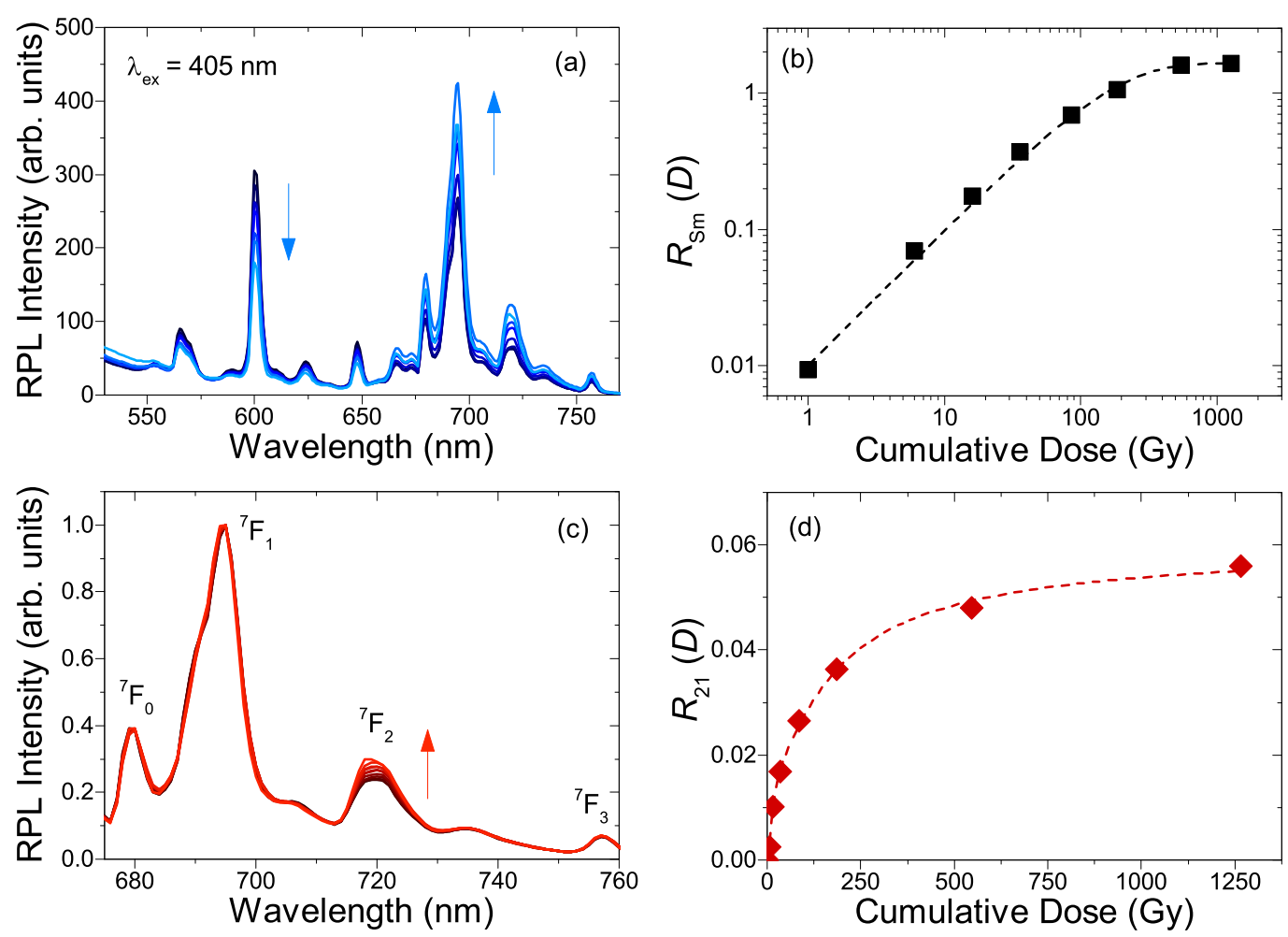

Figure 5.12: (a) PL emissions of $\mathrm{NaMgF}_{3}: \mathrm{Sm}$ where $\lambda_{\mathrm{ex}}=405 \mathrm{~nm}$ for cumulative X-ray doses from 0 Gy to 1266 Gy. The arrows represent the trends for the $\mathrm{Sm}^{3+}$ and $\mathrm{Sm}^{2+}$ emission intensities for increasing total dose. (b) Ratio of the $\mathrm{Sm}^{3+} / \mathrm{Sm}^{2+}$ emissions, $R_{\mathrm{Sm}}(D)$, as a function of cumulative X-ray dose (squares). Also shown is a fit to the data using equation 5.10 (dashed line). (c) PL emissions where $\lambda_{\mathrm{ex}}=405 \mathrm{~nm}$ for cumulative $\mathrm{X}$-ray doses from 0 Gy to $1266 \mathrm{~Gy}$, normalised to the peak intensity of the ${ }^{5} \mathrm{D}_{0} \rightarrow{ }^{7} \mathrm{~F}_{1}$ emission. The arrow indicates the behaviour of the ${ }^{5} \mathrm{D}_{0} \rightarrow{ }^{7} \mathrm{~F}_{2}$ emission intensity for increasing total dose. (d) Ratio of the ${ }^{5} \mathrm{D}_{0} \rightarrow{ }^{7} \mathrm{~F}_{1}$ and ${ }^{5} \mathrm{D}_{0} \rightarrow{ }^{7} \mathrm{~F}_{2}$ emission intensities, $R_{21}(D)$, as a function of cumulative dose (diamonds). The dashed line is a guide to the eye. 
where the ratio would ensure measurements were independent of the collection optics. We define the ratio using only the two peak $\mathrm{Sm}^{2+}$ and $\mathrm{Sm}^{3+}$ emissions as

$$
R_{\mathrm{Sm}}(D)=I_{\mathrm{PL}}(695 \mathrm{~nm}) / I_{\mathrm{PL}}(600 \mathrm{~nm})-R_{\mathrm{Sm}}(0),
$$

where $I_{\mathrm{PL}}(695 \mathrm{~nm})$ is the integrated intensity of the ${ }^{5} \mathrm{D}_{0} \rightarrow{ }^{7} \mathrm{~F}_{1}$ emission of $\mathrm{Sm}^{2+}$ that peaked near $695 \mathrm{~nm}$, and $I_{\mathrm{PL}}(600 \mathrm{~nm})$ is the integrated intensity of the ${ }^{4} \mathrm{G}_{5 / 2} \rightarrow{ }^{6} \mathrm{H}_{7 / 2}$ emission of $\mathrm{Sm}^{3+}$ that peaked near $600 \mathrm{~nm}$.

$R_{\mathrm{Sm}}(D)$ is plotted as a function of cumulative X-ray dose in figure $5.12(\mathrm{~b})$, where the data were collected after the OSL measurements performed in Section 5.2.3 in order to reduce the effect of colour centre absorptions and to demonstrate the viability of the compound for dual OSL/RPL dose monitoring. $R_{\mathrm{Sm}}(D)$ exhibited exponential behaviour where the ratio of emissions saturated for high cumulative doses. It should be noted that similar $\mathrm{Sm}^{3+} \rightarrow \mathrm{Sm}^{2+}$ RPL has been employed for dose monitoring and imaging in Sm-doped glasses $[18,141,166]$, where the dose dependence of $R_{\mathrm{Sm}}(D)$ was described by an equation of the form

$$
R_{\mathrm{Sm}}(D)=R_{0}\left[1-\exp \left(-D / \tau_{\mathrm{RPL}}\right)\right]
$$

where $R_{0}$ is the ratio of Sm emissions at saturation and $\tau_{\mathrm{RPL}}$ is a decay constant. $R_{\mathrm{Sm}}(D)$ was successfully fitted using equation 5.10 where $\tau_{\mathrm{RPL}}=165 \mathrm{~Gy} . R_{\mathrm{Sm}}(D)$ saturated for doses above $500 \mathrm{~Gy}$, indicating that the RPL should be useful over a larger dose range than the OSL, such that cumulative doses could be monitored via RPL while individual doses could be monitored via OSL.

It was of interest to examine the intensities of particular $\mathrm{Sm}^{2+}$ emissions, namely those that occurred via the ${ }^{5} \mathrm{D}_{0} \rightarrow{ }^{7} \mathrm{~F}_{1}$ and ${ }^{5} \mathrm{D}_{0} \rightarrow{ }^{7} \mathrm{~F}_{2}$ transitions (figure 5.12 (c)). Per the discussion in Section 5.1.2, the ${ }^{5} \mathrm{D}_{0} \rightarrow{ }^{7} \mathrm{~F}_{1}$ transition is purely magnetic dipole driven, while the ${ }^{5} \mathrm{D}_{0} \rightarrow{ }^{7} \mathrm{~F}_{2}$ transition is electric dipole driven and therefore highly affected by the crystalline environment, where highly distorted symmetries increase the transition probability $[27,154]$.

The ratio of the two emissions was defined as

$$
R_{21}(D)=I_{\mathrm{PL}}(720 \mathrm{~nm}) / I_{\mathrm{PL}}(695 \mathrm{~nm})-R_{21}(0),
$$

where $I_{\mathrm{PL}}(720 \mathrm{~nm})$ is the integrated intensity of the ${ }^{5} \mathrm{D}_{0} \rightarrow{ }^{7} \mathrm{~F}_{2}$ emission and $R_{21}(0)$ is the ratio of the ${ }^{5} \mathrm{D}_{0} \rightarrow{ }^{7} \mathrm{~F}_{1}$ and ${ }^{5} \mathrm{D}_{0} \rightarrow{ }^{7} \mathrm{~F}_{2}$ emissions as measured prior to irradiation. $R_{21}(D)$ increased as a function of cumulative X-ray dose, shown in figure 5.12 (d), demonstrating 
that those $\mathrm{Sm}^{2+}$ sites formed by $\mathrm{Sm}^{3+}$ electron capture were more distorted than those $\mathrm{Sm}^{2+}$ sites that existed prior to radiation exposure. This is consistent with the interpretation whereby $\mathrm{Sm}^{3+}$ ions with nearby $\mathrm{O}^{2-}$ hole-trapping defects were the structures that underwent the valence conversion, forming $\mathrm{Sm}^{2+}-\mathrm{O}^{-}$structures post-irradiation [151].

\subsubsection{Radioluminescence}
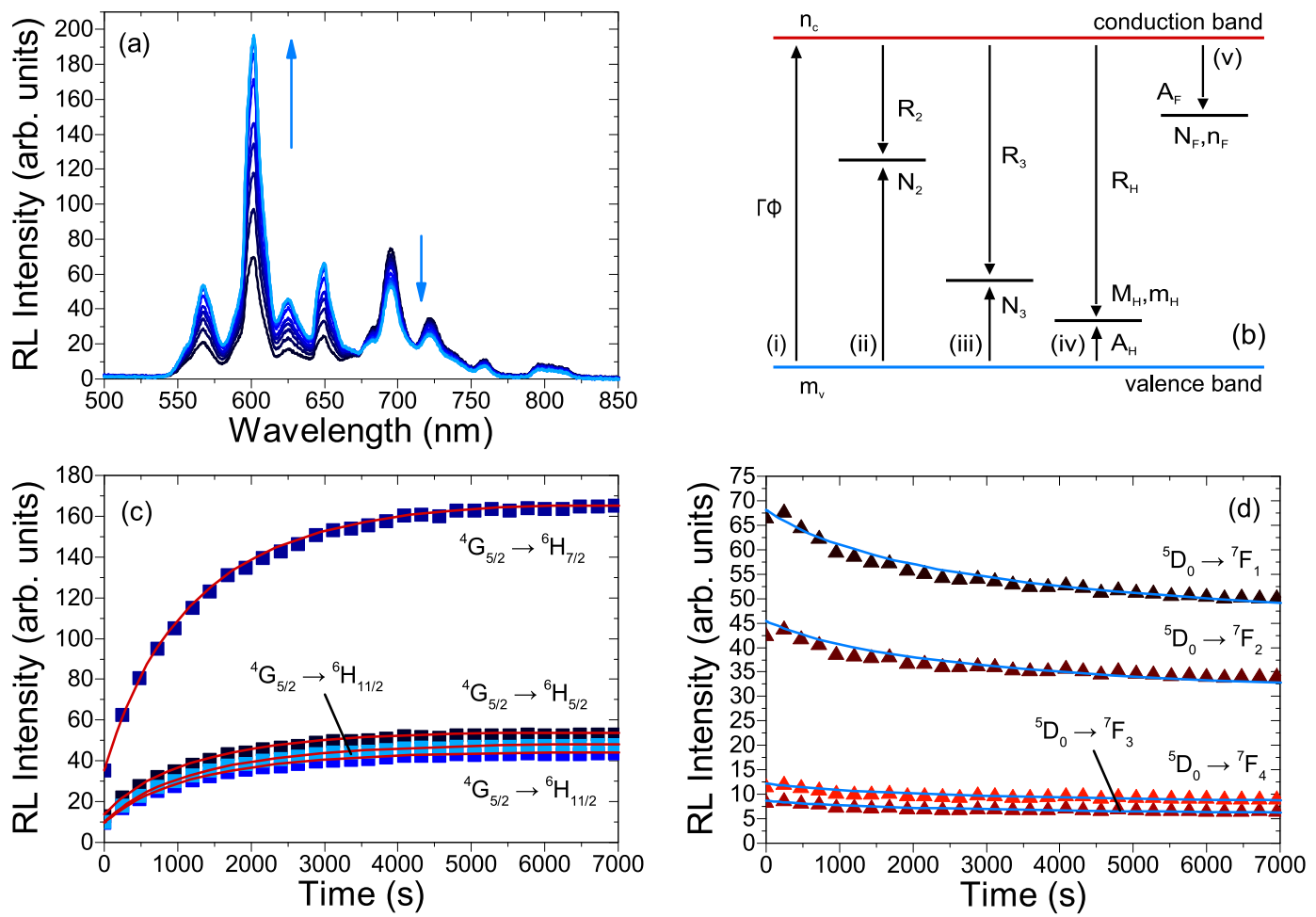

Figure 5.13: (a) RL emission spectra at various times during X-ray irradiation, beginning immediately after the irradiation began and up to 120 minutes of continuous exposure. The arrows indicate the behaviour of the $\mathrm{Sm}^{3+}$ and $\mathrm{Sm}^{2+} \mathrm{RL}$ emission intensities for increasing doses. (b) Schematic of the band model used to model the radioluminescence, where there are two recombination centres, $\mathrm{N}_{2}\left(\mathrm{Sm}^{2+}\right)$ and $\mathrm{N}_{3}\left(\mathrm{Sm}^{3+}\right)$, and two trapping sites, $M_{H}$ and $N_{F}$. For the meanings and values of each parameter, refer to the text. (c) Integrated $\mathrm{RL}$ intensities of each $\mathrm{Sm}^{3+}$ emission as a function of time during continuous $\mathrm{X}$-ray stimulation (squares). The red lines are fits to the data via fitting of the model. (d) Integrated RL intensities of each $\mathrm{Sm}^{2+}$ emission as a function of time during continuous $\mathrm{X}$-ray stimulation (triangles). The blue lines are fits to the data via fitting of the model.

$\mathrm{RL}$ was observed during X-ray exposure from both $\mathrm{Sm}^{3+}$ and $\mathrm{Sm}^{2+}$ sites, shown in figure 5.13 (a). Immediately following the onset of $X$-ray exposure the spectral intensities of the $\mathrm{Sm}^{3+}$ and $\mathrm{Sm}^{2+}$ emissions were approximately equal, similar to the PL prior to 
irradiation when excited at $405 \mathrm{~nm}$. As the total period of X-ray stimulation increased up to 2 hours of continuous exposure the $\mathrm{Sm}^{3+}$ peak intensity of the ${ }^{4} \mathrm{G}_{5 / 2} \rightarrow{ }^{6} \mathrm{H}_{7 / 2}$ emission increased to over $600 \%$ of its initial value. In contrast, the $\mathrm{Sm}^{2+}{ }^{5} \mathrm{D}_{0} \rightarrow{ }^{7} \mathrm{~F}_{1}$ peak emission intensity decreased to $72 \%$ of its initial value. The behaviour of the RL intensities directly contradicted the behaviour of the PL measured after irradiation periods.

In order to better understand this apparent contradiction the RL was modeled using a kinetic model adapted from that used for the $\mathrm{RL}$ of $\mathrm{Al}_{2} \mathrm{O}_{3}: \mathrm{C}$ [60]. A schematic of the model is given in figure 5.13 (b). The model included a single electron trap, primarily representative of F-centres, a single hole trap, representative of the OSL-active hole traps, and two distinct recombination centres $\left(\mathrm{Sm}^{2+}\right.$ and $\left.\mathrm{Sm}^{3+}\right)$. The model assumed that any recombination of conduction band electrons with trapped holes produced luminescence solely from $\mathrm{Sm}^{3+}$, as suggested by the observation of OSL only via $\mathrm{Sm}^{3+}$ sites.

The model used the five interacting processes schematically presented in figure 5.13 (b) to describe the dose dependence of the RL emissions. Process (i) represents the X-ray induced generation of electrons and holes. Processes (ii) and (iii) represent the recombination of the free electrons and holes via the $\mathrm{Sm}^{2+}$ and $\mathrm{Sm}^{3+}$ sites, respectively, upon which RL emission occurs. Process (iv) illustrates the hole trap capturing valence band holes, and the subsequent capture of a free conduction band electron resulting in recombination. In this case, energy transfer to a nearby $\mathrm{Sm}^{3+}$ ion occurs, producing additional $\mathrm{Sm}^{3+} \mathrm{RL}$ emissions. Process (v) represents the capture of electrons by fluorine vacancies, where the trap is stable throughout the irradiation period such that the trapping is considered permanent.

Mathematically, the evolution of the system during irradiation could then be described by a set of coupled differential equations, written

$$
\begin{gathered}
\frac{d n_{\mathrm{F}}}{d t}=n_{\mathrm{c}} A_{\mathrm{F}}\left(N_{\mathrm{F}}-n_{\mathrm{F}}\right), \\
\frac{d n_{\mathrm{c}}}{d t}=\Gamma \dot{D}-\frac{d n_{\mathrm{F}}}{d t}-R_{\mathrm{H}} n_{\mathrm{c}} m_{\mathrm{H}}-R_{2} N_{2} n_{\mathrm{c}} m_{\mathrm{v}}-R_{3} N_{3} n_{\mathrm{c}} m_{\mathrm{v}}, \\
\frac{d m_{\mathrm{v}}}{d t}=\Gamma \dot{D}-m_{\mathrm{v}} A_{\mathrm{H}}\left(M_{\mathrm{H}}-m_{\mathrm{H}}\right)-R_{2} N_{2} n_{\mathrm{c}} m_{\mathrm{v}}-R_{3} N_{3} n_{\mathrm{c}} m_{\mathrm{v}}, \\
\frac{d m_{\mathrm{H}}}{d t}=m_{\mathrm{v}} A_{\mathrm{H}}\left(M_{\mathrm{H}}-m_{\mathrm{H}}\right)-R_{\mathrm{H}} n_{\mathrm{c}} m_{\mathrm{H}},
\end{gathered}
$$

where $n_{\mathrm{F}}, n_{\mathrm{c}}, m_{\mathrm{H}}$, and $m_{\mathrm{v}}$ are the instantaneous concentrations of F-centre electrons, 
conduction band electrons, trapped holes, and holes in the valence band, respectively. $N_{\mathrm{F}}, N_{2}, N_{3}$, and $M_{\mathrm{H}}$ are the total concentrations of F-centre traps (i.e. fluorine vacancies), $\mathrm{Sm}^{2+}$ ions, $\mathrm{Sm}^{3+}$ ions, and hole traps, respectively. $A_{\mathrm{F}}$ and $A_{\mathrm{H}}$ are parameters related to the trapping rates of the F-centre traps and hole traps, respectively. $R_{2}, R_{3}$, and $R_{\mathrm{H}}$ are parameters related to the rates of recombination via the $\mathrm{Sm}^{2+}$ sites, $\mathrm{Sm}^{3+}$ sites, and hole traps, respectively. Finally, $\Gamma$ and $\dot{D}$ are the number of electron-hole pairs produced per unit X-ray dose and unit volume, and the radiation dose rate, respectively.

The RL emission intensities as a function of time will then be proportional to the rates of recombination via each Sm site, and are given by the following equations

$$
\begin{gathered}
I_{\mathrm{RL}}\left(\mathrm{Sm}^{2+}\right)=\alpha_{j 2} R_{2} N_{2} n_{\mathrm{c}} m_{\mathrm{v}} \exp \left(-\sigma_{\mathrm{F} i} n_{\mathrm{F}}\right), \\
I_{\mathrm{RL}}\left(\mathrm{Sm}^{3+}\right)=\left(\alpha_{j 3} R_{3} N_{3} n_{\mathrm{c}} m_{\mathrm{v}}+\beta_{j 3} R_{\mathrm{H}} n_{\mathrm{H}} n_{\mathrm{c}}\right) \exp \left(-\sigma_{\mathrm{F} i} n_{\mathrm{F}}\right),
\end{gathered}
$$

where $\alpha_{j i}$ are dimensionless parameters related to the configuration of the RL collection optics and the probability of RL emission via the distinct electronic transitions, and $\beta_{j i}$ is a similar parameter also related to the probability of $\mathrm{Sm}^{3+}$ OSL emission occurring, as described by the second term of equation 5.17. Per Section 5.2.1 there were several radiation-induced absorptions related to F-type centres in $\mathrm{NaMgF}_{3}: \mathrm{Sm}$ that could affect the RL intensities over time. Most pertinently, the $\mathrm{F}_{3}$-centre absorption at $491 \mathrm{~nm}$ partially overlapped some of the Sm emissions and could reabsorb some Sm emissions, reducing the RL intensities. This effect was accounted for by the exponential decay terms in equations 5.16 and 5.17 , where $\sigma_{\mathrm{F} i}$ is a parameter related to the $\mathrm{F}_{3}$-centre absorption profile and was unique to each Sm emission.

The data presented throughout this chapter provided estimates for several of the parameters required to evaluate the RL model. Firstly, the optical absorption provided an estimate of the F-centre concentration where $N_{\mathrm{F}} \approx 5 \times 10^{17} \mathrm{~cm}^{-3}$. The total concentration of Sm ions was calculated to be $N_{\mathrm{Sm}} \approx 9 \times 10^{18} \mathrm{~cm}^{-3}$, and the fitted magnetic data indicated that the total concentration was divided into approximately $90 \% \mathrm{Sm}^{3+}$ and $10 \% \mathrm{Sm}^{2+}$ such that the concentrations of the ions were $N_{2}=9.8 \times 10^{17} \mathrm{~cm}^{-3}$ and $N_{3}=9 \times 10^{18} \mathrm{~cm}^{-3}$, respectively. All other parameters were initially approximated by those values given for $\mathrm{Al}_{2} \mathrm{O}_{3}: \mathrm{C}$ [60] and adjusted to fit the experimental data. $\Gamma$ was taken to be $10^{14} \mathrm{~cm}^{-3} \mathrm{~Gy}^{-1}$, an order of magnitude lower than the value used for $\mathrm{Al}_{2} \mathrm{O}_{3}: \mathrm{C}$, expected due to the larger band gap of the $\mathrm{NaMgF}_{3}$ host. The values of $R_{2}$ and $R_{3}$ were determined using the initial $\mathrm{RL}$ intensities at $t=0$ for each $\mathrm{Sm}^{2+}$ and $\mathrm{Sm}^{3+}$ transition. 


\begin{tabular}{cccc}
\hline$\Gamma$ & $10^{14} \mathrm{~cm}^{-3} \mathrm{~Gy}^{-1}$ & $R_{3}$ & $1 \times 10^{-11} \mathrm{~cm}^{6} \mathrm{~s}^{-1}$ \\
\hline$\dot{D}$ & $1 \mathrm{Gys}^{-1}$ & $N_{2}$ & $1 \times 10^{18} \mathrm{~cm}^{-3}$ \\
\hline$A_{\mathrm{H}}$ & $3.8 \times 10^{-7} \mathrm{~cm}^{-3} \mathrm{~s}^{-1}$ & $N_{3}$ & $9 \times 10^{18} \mathrm{~cm}^{-3}$ \\
\hline$A_{\mathrm{F}}$ & $4 \times 10^{-8} \mathrm{~cm}^{-3} \mathrm{~s}^{-1}$ & $N_{\mathrm{F}}$ & $5 \times 10^{17} \mathrm{~cm}^{-3}$ \\
\hline$R_{\mathrm{H}}$ & $1.8 \times 10^{-7} \mathrm{~cm}^{-3} \mathrm{~s}^{-1}$ & $M_{\mathrm{H}}$ & $1.1 \times 10^{18} \mathrm{~cm}^{-3}$ \\
\hline$R_{2}$ & $1 \times 10^{-11} \mathrm{~cm}^{6} \mathrm{~s}^{-1}$ & & \\
\hline
\end{tabular}

Table 5.4: Parameters used to fit the RL data using the RL model discussed in the text and mathematically described by equations $5.12-5.15$.

All instantaneous carrier concentrations were set to zero at $t=0$. The values used to fit the RL are presented in table 5.4.

The integrated RL emission intensities, $I_{\mathrm{RL}}$, are shown in figures 5.13 (c) and (d) for the $\mathrm{Sm}^{3+}$ and $\mathrm{Sm}^{2+}$ ions, respectively. The four most intense transitions for each $\mathrm{Sm}$ valence were investigated and the $I_{\mathrm{RL}}$ values were computed by numerically integrating over each emission, using the ranges $546-579 \mathrm{~nm}$ for ${ }^{4} \mathrm{G}_{5 / 2} \rightarrow{ }^{6} \mathrm{H}_{5 / 2}$, $582-617 \mathrm{~nm}$ for ${ }^{4} \mathrm{G}_{5 / 2} \rightarrow{ }^{6} \mathrm{H}_{7 / 2}, 618-639 \mathrm{~nm}$ for ${ }^{4} \mathrm{G}_{7 / 2} \rightarrow{ }^{6} \mathrm{H}_{11 / 2}$, and $642-661 \mathrm{~nm}$ for ${ }^{4} \mathrm{G}_{5 / 2} \rightarrow{ }^{6} \mathrm{H}_{9 / 2}$ for the $\mathrm{Sm}^{3+}$ transitions, and $684-707 \mathrm{~nm}$ for ${ }^{5} \mathrm{D}_{0} \rightarrow{ }^{7} \mathrm{~F}_{1}, 714-750 \mathrm{~nm}$ for ${ }^{5} \mathrm{D}_{0} \rightarrow{ }^{7} \mathrm{~F}_{2}, 751-$ $771 \mathrm{~nm}$ for ${ }^{5} \mathrm{D}_{0} \rightarrow{ }^{7} \mathrm{~F}_{3}$, and $789-822 \mathrm{~nm}$ for ${ }^{5} \mathrm{D}_{0} \rightarrow{ }^{7} \mathrm{~F}_{3}$ for the $\mathrm{Sm}^{2+}$ transitions.

The differential equations 5.12 - 5.15 were then numerically solved using the ode $15 \mathrm{~s}$ function in MATLAB to determine the behaviour of the charge carrier concentrations over time. The RL equations 5.16 and 5.17 were then fitted to the data in figures 5.13 (c) and (d), and the fits are also shown in the figures (solid lines). The values $\alpha_{j i}$ and $\beta_{j i}$ varied for each $\mathrm{Sm}$ emission and correlated with the relative transition intensities. $\sigma_{\mathrm{F} i}$ also varied with each transition. It was largest for the $567 \mathrm{~nm}$ emission and decreased as the peak emission wavelength increased. Consequently, $\sigma_{\mathrm{F} i}$ was zero for all $\mathrm{Sm}^{2+}$ emissions. The behaviour of $\sigma_{\mathrm{F} i}$ was in excellent agreement with the optical absorption data, where the $491 \mathrm{~nm} \mathrm{~F}_{3}$-centre peak extended out to approximately $600 \mathrm{~nm}$ and thus had a negligible effect on the longer wavelength $\mathrm{Sm}^{2+}$ emissions.

The fitted data clearly demonstrated that the proposed model was able to both qual-

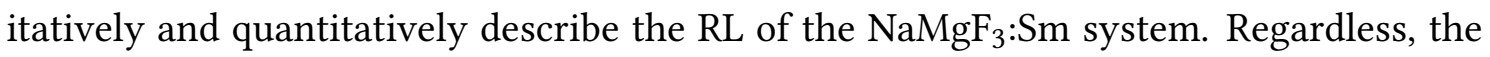
model offered an incomplete description of the system. Firstly, the model did not account for the X-ray induced valence conversion $\mathrm{Sm}^{3+} \rightarrow \mathrm{Sm}^{2+}$. Including this property would result in time-dependent $N_{2}$ and $N_{3}$ values, where $N_{2}$ would increase over time toward a saturation value and $N_{3}$ would decrease inversely. The absence of this term 
was justified to a first approximation by the magnetisation study (Section 5.2.2), where the concentrations of converted Sm ions could not be determined within the experimental uncertainties. A second limitation related to the single electron trap employed that corresponded to F-type centres. It was assumed that the concentration of $\mathrm{F}_{3}$-centres would vary as a linear function of the concentration of F-centres. While the assumption proved viable, the actual processes of defect formation and agglomeration were likely more complicated, and the accuracy of the fitting could potentially be improved by including several additional traps in the model.
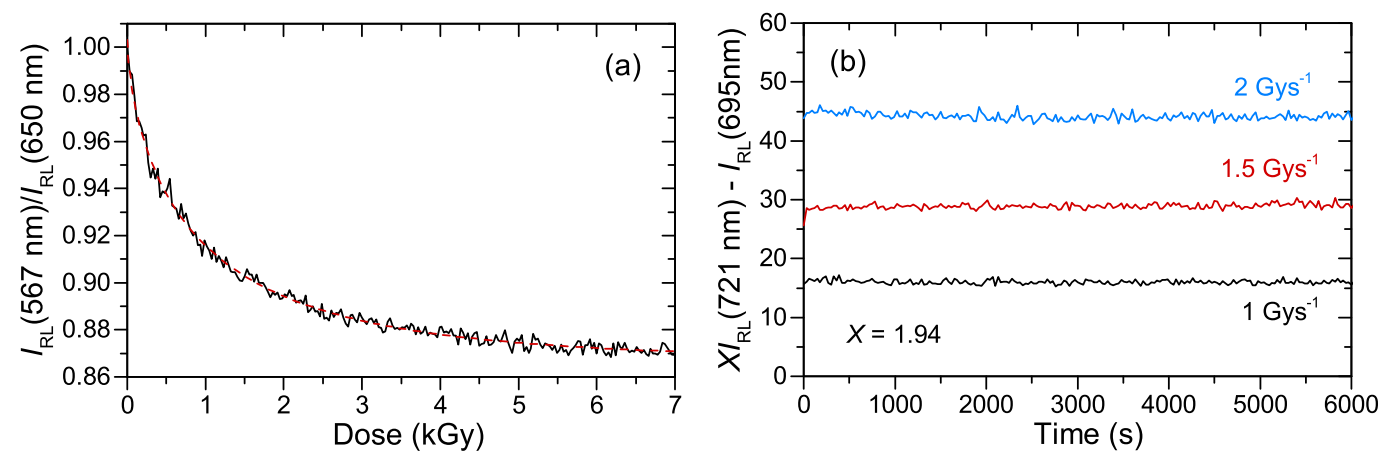

Figure 5.14: (a) Normalised ratio of the integrated RL intensities for the $\mathrm{Sm}^{3+}$ emissions at $567 \mathrm{~nm}$ and $650 \mathrm{~nm}$ (black). A stretched exponential fit to the data is shown (red dashed). (b) Plot of equation 5.19 for dose rates $1 \mathrm{Gys}^{-1}$ (black), $1.5 \mathrm{Gys}^{-1}$ (red), and 2 $\mathrm{Gys}^{-1}$ (blue).

Despite the aforementioned limitations, the RL model was sufficient in describing the behaviour of the Sm RL emissions over time, such that some consequences of the model may now be explored. The F-type centre concentrations could be indirectly measured by monitoring the relative intensities of the $\mathrm{Sm}^{3+} \mathrm{RL}$ emissions. $\mathrm{F}_{3}$-centre absorptions most prominently affected the ${ }^{4} \mathrm{G}_{5 / 2} \rightarrow{ }^{6} \mathrm{H}_{5 / 2}$ transition and minimally affected the ${ }^{4} \mathrm{G}_{5 / 2}$ $\rightarrow{ }^{6} \mathrm{H}_{9 / 2}$ transition. Thus, behaviour of the ratio of the integrated intensities for each emission, written $I_{\mathrm{RL}}(567 \mathrm{~nm}) / I_{\mathrm{RL}}(650 \mathrm{~nm})$, should be characteristic of the concentration of $\mathrm{F}_{3}$-centres. This ratio is plotted in figure 5.14 (a) as a function of X-ray dose.

The ratio $I_{\mathrm{RL}}(567 \mathrm{~nm}) / I_{\mathrm{RL}}(650 \mathrm{~nm})$ decreased with radiation dose as expected due to the increased population of $\mathrm{F}_{3}$-centres at higher doses. The decay could be fitted to a stretched exponential decay of the form

$$
I_{\mathrm{RL}}(567 \mathrm{~nm}) / I_{\mathrm{RL}}(650 \mathrm{~nm})=I_{0}+I \times \exp (-D / \tau)^{\beta},
$$

where $I_{0}$ is the ratio when the $\mathrm{F}_{3}$-centre concentration saturates, $I$ is a prefactor, $\beta$ is a 
stretching parameter, and $\tau$ a decay constant. The fit yielded the parameters $\beta=0.65$ and $\tau=0.96 \mathrm{kGy}$. Stretched exponential behaviour suitably describes several luminescence phenomena, including OSL decays in the case of high retrapping rates [167], and PL decays in the case of distributed decay rates [168]. The latter interpretation may be extended to the data presented here, where the relative locations of the $\mathrm{F}_{3}$-centres and $\mathrm{Sm}^{3+}$ ions resulted in a distribution of absorption probabilities that led to stretched exponential behaviour.

As F-type centre concentrations are typically measured using optical absorption data and via Smakula's equation, it is of interest to develop novel means of monitoring defect concentrations. Provided an initial calibration were performed, the ratio $I_{\mathrm{RL}}(567$ $\mathrm{nm}) / I_{\mathrm{RL}}(650 \mathrm{~nm})$ should provide a means of monitoring the $\mathrm{F}_{3}$-centre concentrations in $\mathrm{NaMgF}_{3}: \mathrm{Sm}$, which could then be correlated with the received radiation dose.

The $\mathrm{RL}$ of the $\mathrm{Sm}^{2+}$ ions also presented a novel means of monitoring radiation doses by exploiting an experimentally observed relationship between the distinct $\mathrm{Sm}^{2+} \mathrm{RL}$ emissions. There existed a linear relationship between the $\mathrm{Sm}^{2+}$ emissions that could be described by an equation of the form

$$
I_{\mathrm{RL}}\left(\mathrm{Sm}^{2+}, j\right)=X \times I_{\mathrm{RL}}\left(\mathrm{Sm}^{2+}, j^{\prime}\right)-C,
$$

where $j$ and $j$ represent distinct $\mathrm{Sm}^{2+}$ emissions and $X$ and $C$ are constants. Letting $I_{\mathrm{RL}}\left(\mathrm{Sm}^{2+}, j\right)=I_{\mathrm{RL}}(695 \mathrm{~nm})$ and $I_{\mathrm{RL}}\left(\mathrm{Sm}^{2+}, j^{\prime}\right)=I_{\mathrm{RL}}(721 \mathrm{~nm})$, a straightforward rearrangement of equation 5.18 leads to

$$
\left[X \times I_{\mathrm{RL}}(721 \mathrm{~nm})\right]-I_{\mathrm{RL}}(695 \mathrm{~nm})=C,
$$

which has no direct dependence on the total radiation dose. Equation 5.19 was evaluated using the experimental data and the results are plotted in figure 5.14 (b) for the different dose rates $1 \mathrm{Gys}^{-1}, 1.5 \mathrm{Gys}^{-1}$, and $2 \mathrm{Gys}^{-1}$ where $X=1.94$. The parameter $C$ increased approximately linearly with the dose rate, and consequently equation 5.19 could be used for real-time monitoring of radiation doses and dose rates without a priming dose and without accounting for dose history. However, it should be noted that equation 5.19 may fail for higher total doses and dose rates, where increased concentrations of $\mathrm{F}_{3}$-centres could affect the $\mathrm{Sm}^{2+}$ emissions. 


\subsubsection{Thermal Quenching of the Luminescence}

According to the HRBE diagram constructed for $\mathrm{NaMgF}_{3}: \mathrm{Ln}$ (figure 5.8) the ground state of the $\mathrm{Sm}^{2+}$ ion was located $4.44 \mathrm{eV}$ below the conduction band edge and therefore acted as a very stable electron trap. The first $5 d$ level of $\mathrm{Sm}^{2+}$ was located $2.85 \mathrm{eV}(435 \mathrm{~nm})$ above the $4 f$ ground state, corresponding to a location $1.59 \mathrm{eV}(778 \mathrm{~nm})$ below the conduction band edge. Per the previous analysis (Section 5.1.5) there was a significant uncertainty in these values up to approximately $\pm 0.5 \mathrm{eV}$, largely derived from the difficulty in accurately measuring trap depths using optical stimulations and the imprecise placement of the $\mathrm{O}^{2-}$ level. It was therefore of interest to investigate an alternative means of placing the $\mathrm{Ln}^{2+}$ trap depths and improving the accuracy of the HRBE diagram.

As the $\mathrm{Sm}^{2+} 5 d$ level is $\sim 2.85 \mathrm{~nm}$ above the $4 f$ ground state and $\sim 1.59 \mathrm{eV}$ below the conduction band, we expected that a combination of optical and thermal stimulation would result in electronic excitations from the $\mathrm{Sm}^{2+}$ ground state to the conduction band. Conduction band thermal quenching of the $\mathrm{Sm}^{2+}$ luminescence could then occur, where the eventual recombination of the $\left(\mathrm{Sm}^{3+}+\mathrm{e}_{\mathrm{CB}}^{-}\right)$centre would be non-radiative. This process has been well established for the $4 f \rightarrow 5 d$ transitions of the $\mathrm{Eu}^{2+}$ ion in a variety of host compounds [169-171]. In contrast, very little data existed for the $\mathrm{Sm}^{2+}$ ion, though one study observed the photo-thermal ionisation of $\mathrm{Sm}^{2+}$ below room temperature in $\mathrm{YPO}_{4}: \mathrm{Ce}, \mathrm{Sm}[172]$.

In order to investigate the thermal quenching of the $\mathrm{Sm}^{2+}$ luminescence in $\mathrm{NaMgF}_{3}: \mathrm{Sm}$, the TL equipment detailed in Section 3.2.6 was employed, where a powdered sample was stimulated using $448 \mathrm{~nm}$ LED light and heated using the TL apparatus. The $448 \mathrm{~nm}$ (2.77 eV) LED stimulated directly into the $5 d$ band and the PL spectra were collected using the USB2000+ CCD spectrometer after filtering the signal through a RG665 $665 \mathrm{~nm}$ long-pass filter. The LED intensity was monitored using a Thorlabs PDA100A Si photodetector in order to ensure no significant intensity fluctuations would affect the PL spectra. The intensity at the sample was constant at approximately $0.14 \mathrm{~mW} / \mathrm{cm}^{2}$ for all measurements. After adhering the powdered sample to the TL finger the temperature was cycled from $300 \mathrm{~K}$ to $673 \mathrm{~K}$ eight times in order to evaporate any excess silicone adhesive and to homogenise the powder. The final four scans did not vary regarding initial and final luminescence intensities, such that the experimental apparatus was considered primed for subsequent analyses.

The PL spectra under $448 \mathrm{~nm}$ stimulation for temperatures from $300 \mathrm{~K}$ to $680 \mathrm{~K}$, ramped at $1 \mathrm{~K} / \mathrm{s}$, are shown in figure 5.15 (a). The $\mathrm{Sm}^{2+}$ emissions were the same as those observed in the PL and RL, peaking at $692 \mathrm{~nm}, 720 \mathrm{~nm}, 755 \mathrm{~nm}$, and $800 \mathrm{~nm}$. The peak 

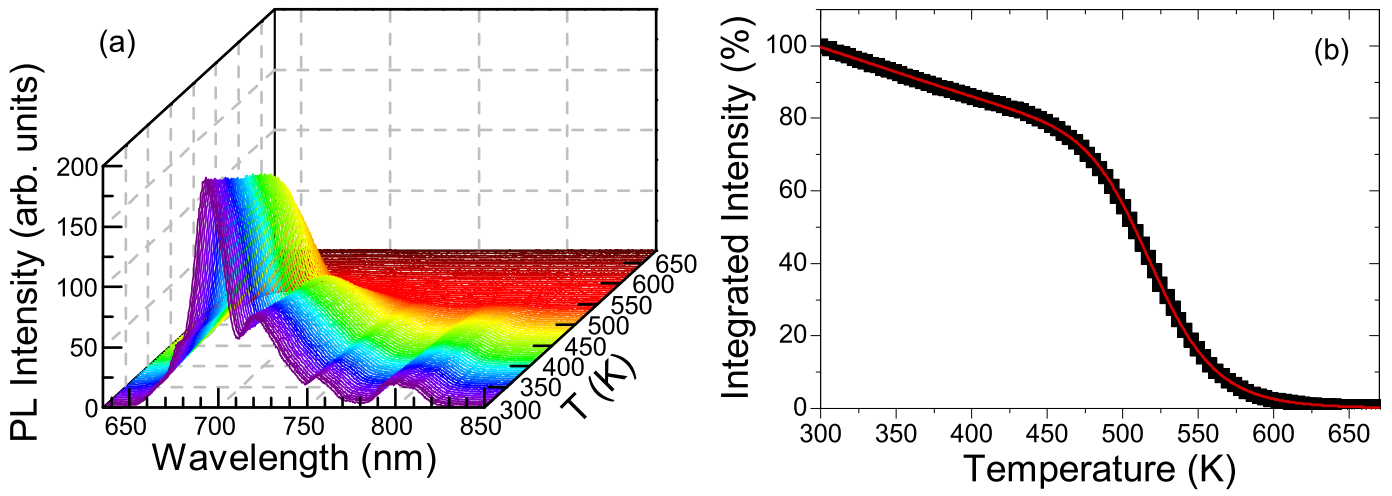

Figure 5.15: (a) PL emission spectra of $\mathrm{NaMgF}_{3}: \mathrm{Sm}$ during $448 \mathrm{~nm}$ stimulation at temperatures ranging from $300 \mathrm{~K}$ to $680 \mathrm{~K}$ ramped at $1 \mathrm{~K} / \mathrm{s}$. (b) Integrated intensity of the $\mathrm{Sm}^{2+}$ ${ }^{5} \mathrm{D}_{0} \rightarrow{ }^{7} \mathrm{~F}_{1} \mathrm{PL}$ emissions as a function of temperature. The red line is a fit to equation 5.20 .

emission intensities for all transitions decreased as the temperature was increased, at the same rate. For temperatures approaching $650 \mathrm{~K}$, the $\mathrm{Sm}^{2+}$ luminescence was entirely quenched. The $\mathrm{Sm}^{2+}$ luminescence was restored to its initial intensity upon cooling to room temperature.

The integrated intensity, $I_{\mathrm{PL}}(T)$, of the ${ }^{5} \mathrm{D}_{0} \rightarrow{ }^{7} \mathrm{~F}_{1}$ emission taken from $680 \mathrm{~nm}$ $710 \mathrm{~nm}$ is plotted in figure 5.15 (b) as a function of temperature. There were at least two components in the thermal quenching curve, where a slow decrease occurred up to approximately $450 \mathrm{~K}$, followed by rapid quenching for higher temperatures. The two component quenching could be described by the following equation

$$
I_{\mathrm{PL}}(T)=I_{0} /\left[1+\Gamma_{1} \exp \left(-\varepsilon_{1} / \mathrm{k}_{\mathrm{B}} T\right)+\Gamma_{2} \exp \left(-\varepsilon_{2} / \mathrm{k}_{\mathrm{B}} T\right)\right],
$$

where $I_{0}$ is the theoretical PL intensity at $0 \mathrm{~K}, \mathrm{k}_{\mathrm{B}}$ is the Boltzmann constant, $\Gamma_{i}$ are prefactors related to the non-radiative transition probabilities, and $\varepsilon_{i}$ are the activation energies of each quenching component. The data in figure 5.15 (b) were fitted to equation 5.20 with the following parameters: $\varepsilon_{1}=0.09 \pm 0.01 \mathrm{eV}, \Gamma_{1}=3.8 \pm 0.2$, and $\varepsilon_{2}=1.2 \pm$ $0.1 \mathrm{eV}, \Gamma_{2}=(1.9 \pm 0.5) \times 10^{11}$.

The existence of two quenching components was unsurprising. The lower temperature component $(i=1)$ had a small activation energy and was most likely due to nonradiative decay to the ground state from selected states within the $4 f^{5} 5 d^{1}$ configuration. This implied that some fraction of the luminescence at room temperature was quenched, though the low transition probability as given by $\Gamma_{1}$ justified the observation of intense luminescence at room temperature. The mechanism can be described as follows: the 
$\mathrm{Sm}^{2+}$ ion was excited into the first $4 f^{5} 5 d^{1}$ level. Some excited electrons directly decayed to the $4 f^{6}\left({ }^{7} \mathrm{~F}_{0}\right)$ ground state, for which no emission was observed. The remaining electrons non-radiatively decayed to the $4 f^{6}\left({ }^{5} \mathrm{D}_{0}\right)$ excited state, from which they radiatively decayed to the various $4 f^{6}\left({ }^{7} \mathrm{~F}_{\mathcal{f}}\right)$ states. These processes occurred for temperatures below $\sim 450 \mathrm{~K}$ and could be used for all-optical temperature sensing. The thermal quenching driven by the $i=1$ component resulted in an approximately linear decrease in the luminescence intensity of $0.13 \% / \mathrm{K}$.

The higher temperature quenching component $(i=2)$ was characteristic of the expected conduction band quenching mechanism. The activation energy of $\varepsilon_{2}=1.2 \pm 0.1$ $\mathrm{eV}$ is in reasonable agreement with the energy from the first $5 d$ level to the conduction band edge as predicted by the HRBE diagram. As the energy of the first $5 d$ level was clearly established via PL excitation data at $\varepsilon_{\mathrm{d}}=2.8 \mathrm{eV}$, the trap depth of $\mathrm{Sm}^{2+}$ was then calculated to be $\varepsilon_{\mathrm{d}}+\varepsilon_{2}=4.0 \mathrm{eV}$. The high value of $\Gamma_{2}$ indicated that conduction band quenching was the preferred decay pathway when possible, leading to the total quenching of the luminescence for high temperatures.
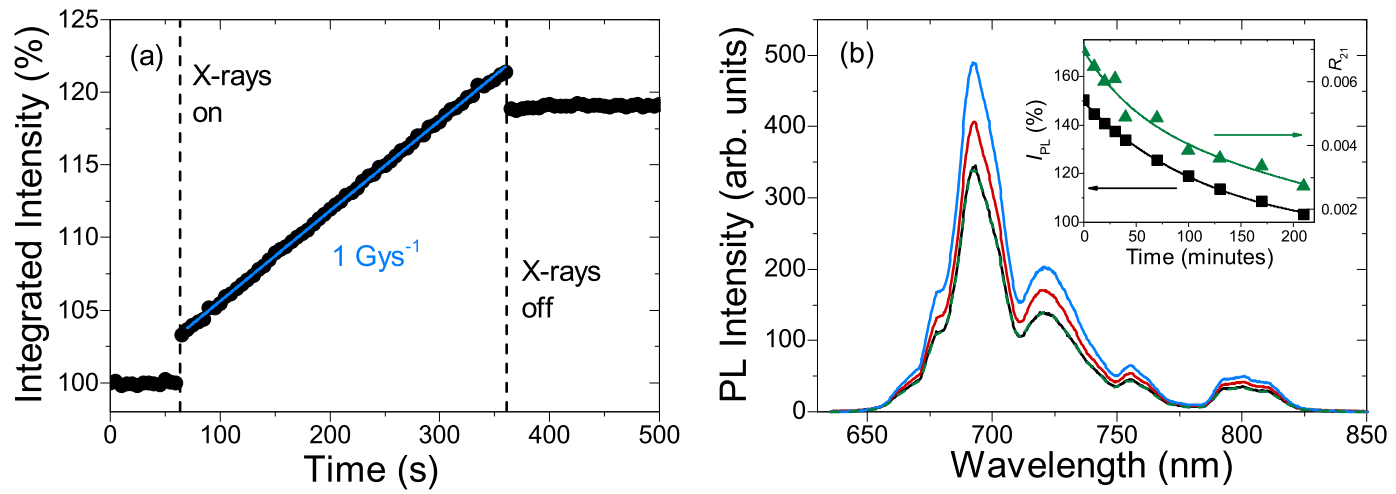

Figure 5.16: (a) Integrated intensity of the PL emissions of $\mathrm{Sm}^{2+}$ during $448 \mathrm{~nm}$ stimulation, where the sample was exposed to X-rays from $t=60 \mathrm{~s}$ to $t=360 \mathrm{~s}$ (black circles). The blue line is a linear fit over the period of irradiation. (b) Room temperature PL emission spectra obtained prior to irradiation (black), after an X-ray dose of 300 Gy (red), after 40 minutes at $573 \mathrm{~K}$ without any optical stimulation (blue), and after 210 minutes at $573 \mathrm{~K}$ with continuous $448 \mathrm{~nm}$ optical stimulation (green dashed). Inset: Integrated PL intensity normalised to the intensity prior to irradiation, for different times during which the sample was held at $573 \mathrm{~K}$ and stimulated with $448 \mathrm{~nm}$ light (black squares). Also shown is an exponential decay fit to the data (black line). The site distortion ratio, $R_{21}(D)$, is also shown (green triangles). The green line is a guide to the eye.

The conduction band quenching of the $\mathrm{Sm}^{2+}$ luminescence can be considered a photothermal ionisation process, where optical stimulation resulted in excitations from the 
$4 f$ ground state to the first excited $5 d$ state, and concurrent thermal stimulation ionised the $5 d$ electron to the conduction band. To confirm that this is the correct physical description of the quenching process, the effects of temperature on the $\mathrm{Sm}^{3+} \rightarrow \mathrm{Sm}^{2+} \mathrm{RPL}$ process were investigated.

Figure 5.16 (a) shows the real-time RPL where the integrated intensity of the $\mathrm{Sm}^{2+}$ emissions was monitored as a function of time before, during, and after X-ray stimulation. At $60 \mathrm{~s}$ the sample was exposed to the X-ray beam at a dose rate of $1 \mathrm{Gys}^{-1}$ and the intensity of the $\mathrm{Sm}^{2+}$ luminescence increased as $\mathrm{Sm}^{3+}$ trapped electrons to form $\mathrm{Sm}^{2+}$ sites. The integrated PL intensity increased linearly over the period of irradiation by $18 \%$ $(0.06 \% / G y)$. The X-ray stimulation was ceased at $360 \mathrm{~s}$, after which the $\mathrm{Sm}^{2+} \mathrm{PL}$ was approximately constant. The discontinuities at $60 \mathrm{~s}$ and $360 \mathrm{~s}$ occurred due to contributions from the RL of $\mathrm{Sm}^{2+}$, as detailed in Section 5.2.5.

If photothermal ionisation was the process by which the $\mathrm{Sm}^{2+}$ luminescence was quenched, the process should also revert the X-ray produced $\mathrm{Sm}^{2+}$ back to $\mathrm{Sm}^{3+}$ as the conduction band electrons recombined with nearby trapped holes. In order to test this, several temperature cycles were performed with and without $448 \mathrm{~nm}$ optical stimulation. The effect of the photothermal stimulations on the PL of $\mathrm{Sm}^{2+}$ are shown in figure 5.16 (b). Initially, the sample was ramped to $573 \mathrm{~K}$ at $5 \mathrm{~K} / \mathrm{s}$ in the absence of optical stimulation and held for 10 minutes, after which it was allowed to cool to room temperature. The PL intensity increased after this initial stage due to two main processes: (i) F-type centres were thermally bleached at this temperature, producing additional conduction band electrons that could be captured by $\mathrm{Sm}^{3+}$ ions to produce an increased $\mathrm{Sm}^{2+}$ concentration, and (ii) the thermal bleaching of F-type centres reduced the optical absorptions of the centres and resulted in a higher population of $\mathrm{Sm}^{2+}$ ions absorbing the stimulation light. This heating stage was repeated several times up to a total time of 40 minutes, though the PL intensity did not change after the first heating step. As no reduction in $\mathrm{Sm}^{2+}$ intensity occurred, the thermal stimulation was insufficient in exciting any $\mathrm{Sm}^{2+}$ electrons to the conduction band, as expected.

The sample was then ramped to $573 \mathrm{~K}$ at $5 \mathrm{~K} / \mathrm{s}$ and held for 10 minutes, while stimulating with $448 \mathrm{~nm}$ light. After cooling to room temperature a reduction in the $\mathrm{Sm}^{2+}$ PL intensity was observed. This step was repeated up to a total time of 210 minutes, after which the PL intensity was restored to that observed prior to irradiation. The PL intensity decreased exponentially for longer stimulation times, as illustrated in the inset to figure 5.16 (b). Ultimately, these data indicated that photothermal ionisation was the process by which the luminescence was quenched, as the X-ray produced population of 
$\mathrm{Sm}^{2+}$ ions was reverted to $\mathrm{Sm}^{3+}$.

The above interpretation was confirmed by analysing the relative site distortions of the $\mathrm{Sm}^{2+}$ ions. It was shown in Section 5.2.4 that the ratio of the ${ }^{5} \mathrm{D}_{0} \rightarrow{ }^{7} \mathrm{~F}_{2}$ and ${ }^{5} \mathrm{D}_{0} \rightarrow$ ${ }^{7} \mathrm{~F}_{1}$ emissions, $R_{21}$, could be used as a measure of the relative site distortion, where $R_{21}$ is defined by equation 5.11. Furthermore, the X-ray produced $\mathrm{Sm}^{2+}$ was demonstrated to be more distorted than the pre-existing $\mathrm{Sm}^{2+}$ population. The inset to figure 5.16 (b) shows the behaviour of $R_{21}$ as a function of photothermal stimulation time, where $R_{21}$ decreased as the converted $\mathrm{Sm}^{2+}$ ions were restored to $\mathrm{Sm}^{3+}$. The data clearly demonstrate that the distorted $\mathrm{Sm}^{2+}$ ions produced during the irradiation were the sites that were reconverted to $\mathrm{Sm}^{3+}$.
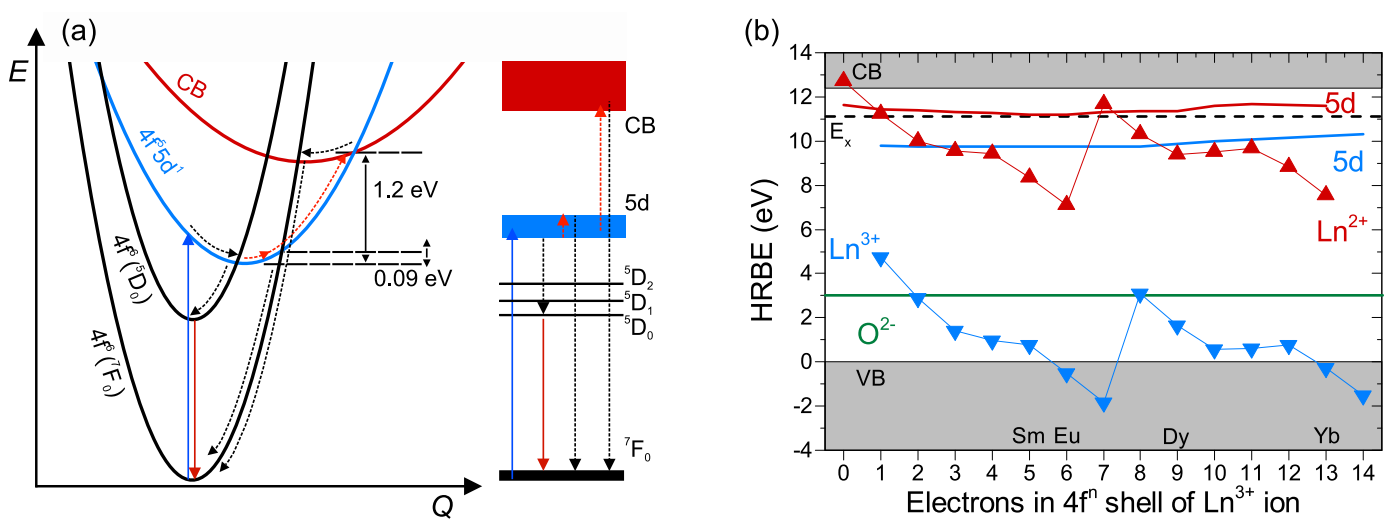

Figure 5.17: (a) Schematic configurational coordinate diagram describing the radiative and non-radiative transitions that occurred in $\mathrm{NaMgF}_{3}: \mathrm{Sm}$. Solid arrows represent optical transitions, black dashed arrows represent non-radiative decays, and orange dashed arrows represent thermally driven processes. (b) Revised HRBE diagram where the position of the $\mathrm{O}^{2-}$ level is better defined (see figure 5.8).

The thermal quenching data allowed for the construction of a configurational coordinate diagram that detailed the various possible electronic decay pathways of the $\mathrm{Sm}^{2+}$ ion in the $\mathrm{NaMgF}_{3}: \mathrm{Sm}$ system, shown in figure 5.17 (a). Straightforward optical stimulation at $448 \mathrm{~nm}$ could excite electrons from the $4 f^{6}$ ground state to the first excited $4 f^{5} 5 d^{1}$ state. In the absence of thermal effects, the excited electrons would primarily non-radiatively decay to the excited ${ }^{5} \mathrm{D}_{0}$ state within the $4 f^{5}$ configuration, after which they would radiatively decay to the various ${ }^{7} \mathrm{~F}_{\mathcal{f}}$ states. A small thermal contribution could instead result in the excitation of the $4 f^{5} 5 d^{1}$ electrons to a slightly higher energy level, whereupon they could access a non-radiative pathway to the ${ }^{7} \mathrm{~F}_{0}$ ground state. The activation energy for this process was calculated to be $0.09 \mathrm{eV}$. Significant thermal contributions could instead excite the $4 f^{5} 5 d^{1}$ electrons to the conduction band, where- 
upon they could access a preferential non-radiative pathway to the ${ }^{7} \mathrm{~F}_{0}$ ground state. The activation energy for this photothermal ionisation process was calculated to be 1.2 $\mathrm{eV}$. Additionally, as the photothermal ionisation involved conduction band states, the process could revert the X-ray induced valence conversion $\mathrm{Sm}^{3+} \rightarrow \mathrm{Sm}^{2+}$, where the electron in the conduction band could recombine with a localised trapped hole.

The data presented in this section allowed for the production of a revised HRBE diagram where the $\mathrm{Sm}^{2+}$ level was better defined at $4.0 \mathrm{eV}$ below the conduction band (figure 5.17 (b). The revised diagram is consistent with all previously presented data within uncertainty, where the $\mathrm{O}^{2-}$ level was placed $3 \mathrm{eV}$ above the top of the valence band. The analysis of thermal quenching based on the photothermal ionisation of divalent $\mathrm{Ln}^{2+}$ ions represents a novel technique by which one can estimate the $\mathrm{Ln}^{2+}$ trap depths in any Ln-doped compound, as required for the development of HRBE diagrams and of great importance in the luminescence studies of Ln-doped materials.

\subsubsection{Radiation-induced Conduction}

Given the recent development of diamond-based dosimeters [50-52, 173], where realtime radiation doses are monitored using electrical conduction, it was of interest to investigate similar phenomena in the $\mathrm{NaMgF}_{3}: \mathrm{Sm}$ system. A sample of polycrystalline $\mathrm{NaMgF}_{3}: \mathrm{Sm}$ was cut to dimensions $10 \mathrm{~mm} \times 8 \mathrm{~mm} \times 1 \mathrm{~mm}$, and thin wires were attached to both faces using conductive Epotek H20E silver epoxy. The electrodes on each face were approximately equivalent with areas of $\sim 20 \mathrm{~mm}^{2}$. The sample was then connected in series to a Keithley 617 Electrometer such that a variable voltage could be applied and the electric current monitored simultaneously. The sample was mounted in the beamline of the X-ray generator and an optical fibre was attached, such that the real-time RL could be detected concurrent with the electrical measurements.

A simplified schematic of the configuration is shown in figure 5.18 (a). The sample effectively functioned as a parallel plate capacitor, where the $\mathrm{NaMgF}_{3}: \mathrm{Sm}$ acted as the dielectric medium between the silver electrodes. Upon application of a voltage, opposite charges collected at either electrode over time until the capacitor was fully charged. The non-zero resistance of the compound resulted in a small leakage current. Additionally, the sample volume became polarised over time, producing an additional contribution to the current. These phenomena resulted in the charging and discharging curves shown in figures 5.18 (b) and (c), where similar behaviours have been observed in other materials $[174,175]$, though with relatively little discussion.

The system could be modeled as a leaky capacitor with an additional volume po- 

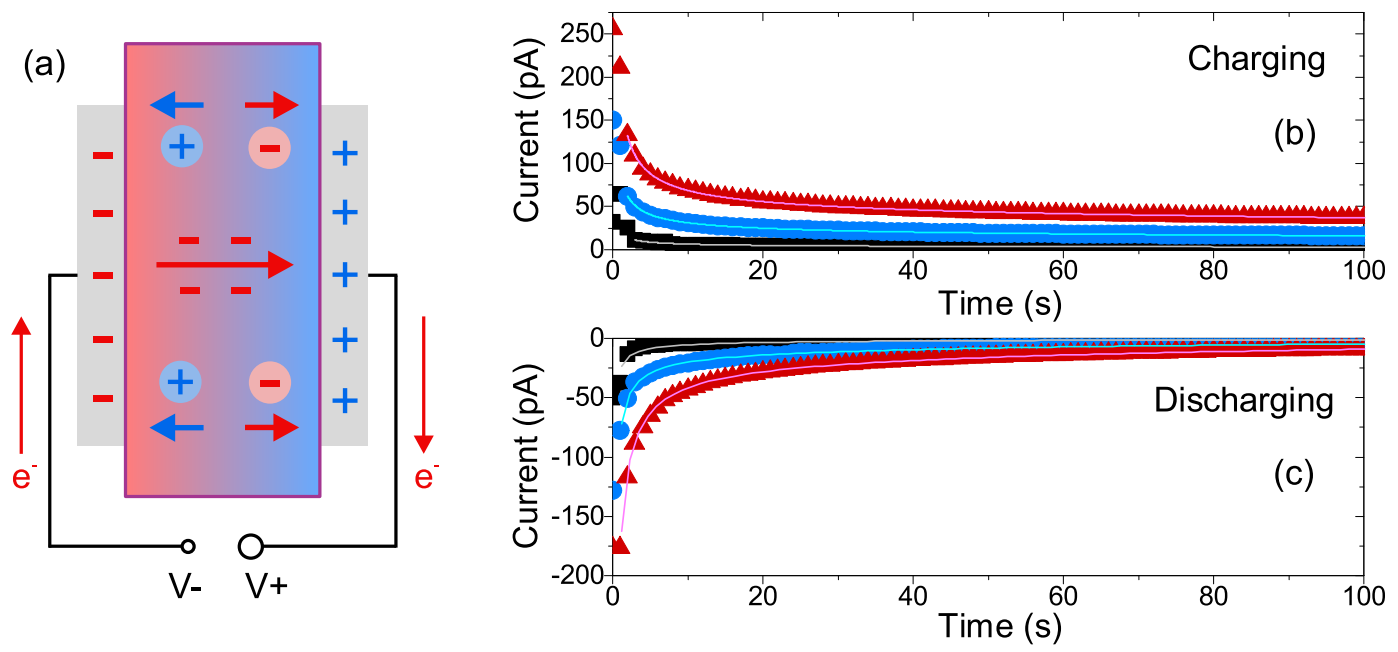

Figure 5.18: (a) Schematic illustration of the likely conduction mechanisms of $\mathrm{NaMgF}_{3}: \mathrm{Sm}$ in the absence of external stimulation. Red arrows represent the flow of electrons and negatively charged ions/defects and blue arrows represent the flow of positively charged ions/defects (b) Current vs. time immediately after a voltage was applied, where $V=10 \mathrm{~V}$ (black), $V=50 \mathrm{~V}$ (blue), and $V=100 \mathrm{~V}$ (red). The solid lines are fits to the data using equation 5.21. (c) Current vs. time immediately after the applied voltage was removed, where the initial voltages were $V=10 \mathrm{~V}$ (black), $V=50 \mathrm{~V}$ (blue), and $V=$ $100 \mathrm{~V}$ (red). The solid lines are fits to the data using equation 5.22.

larisation contribution. Consequently, the equations describing the current, $i(t)$, while charging and discharging were

$$
i(t)=A \times(1+B \times \exp (-t / \tau))+D t^{-\beta},
$$

and

$$
i(t)=A \times \exp (-t / \tau)+D t^{-\beta},
$$

respectively. The parameters $A, B, D, \tau$, and $\beta$ are constants that could be related to physical values. The steady state leakage current that was constant as $t \rightarrow \infty$ implied that $A=V_{0} / R_{\mathrm{T}}$, where $V_{0}$ was the applied voltage and $R_{\mathrm{T}}$ was the total resistance of the circuit. The constant $B$ is proportional to the resistance of the circuit. The decay constant $\tau$ should be related to the capacitance of the system, $C$, such that $\tau \approx R_{\mathrm{T}} C$. Finally, $D$ and $\beta$ were constants related to the volume polarisation of the sample as described by the Curie-von Schweidler law [176], such that $D \propto V_{0}$ and $0<\beta<1$. The data of figures 5.18 (b) and (c) were fitted to equations 5.21 and 5.22, respectively. The calculated resistance of the system was then $R_{\mathrm{T}} \approx 5 \times 10^{12} \Omega$, and the resistivity of the $\mathrm{NaMgF}_{3}: \mathrm{Sm}$ sample 
was $\rho \approx 1 \times 10^{13} \Omega \mathrm{cm}$.
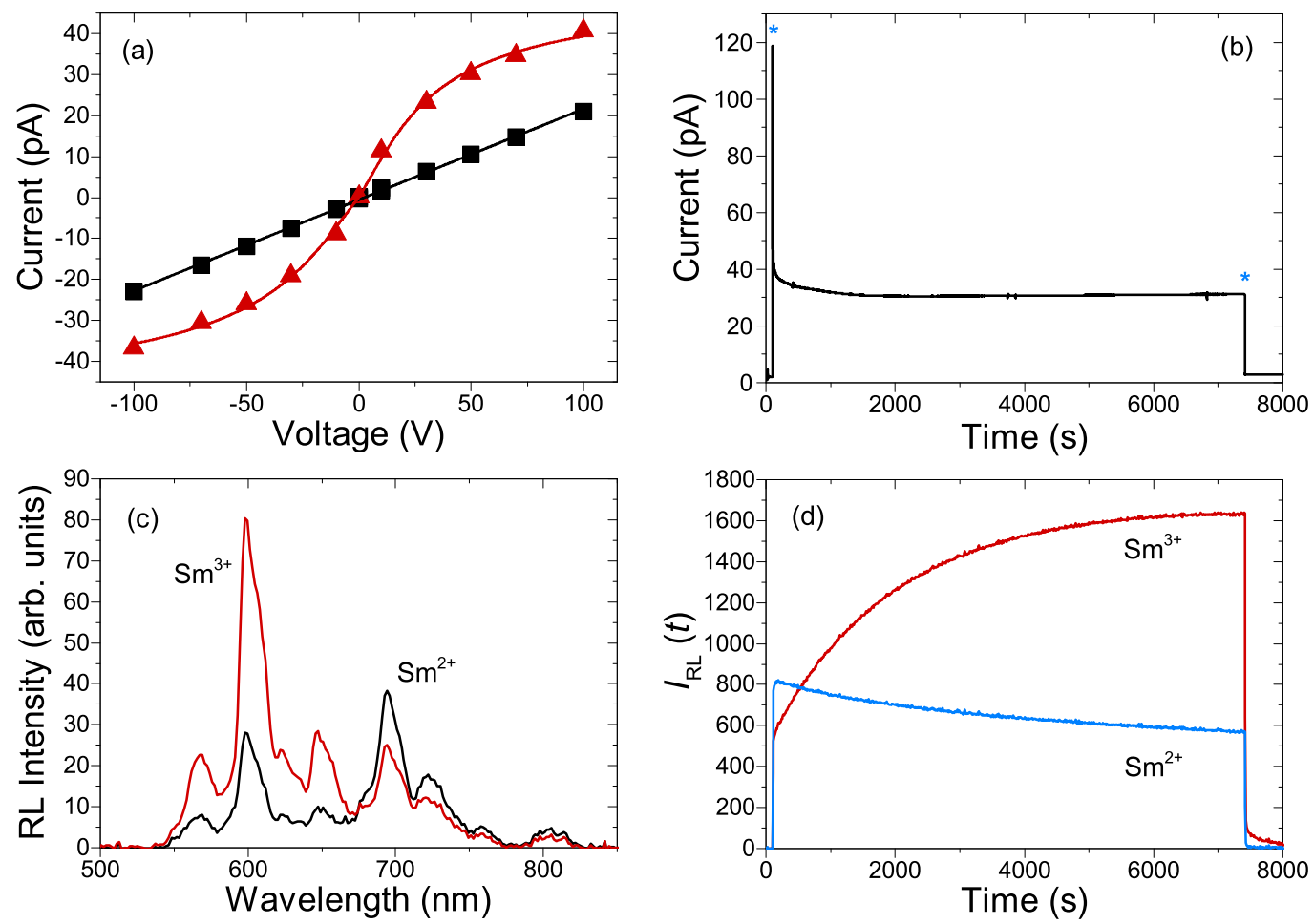

Figure 5.19: (a) Current-voltage behaviour for $\mathrm{NaMgF}_{3}: \mathrm{Sm}$ in the absence of irradiation (black squares) and during X-ray irradiation (red triangles). The black line is a linear fit and the red curve is a fit to equation 5.23. (b) Current as a function of time, where the sample was exposed to X-ray irradiation continuously from $t=100 \mathrm{~s}$ to $t=7410 \mathrm{~s}$. (c) RL spectra obtained after $100 \mathrm{~s}$ of exposure (black) and after $7200 \mathrm{~s}$ of exposure (red). (d) Integrated RL intensities of the peak $\mathrm{Sm}^{3+}$ emission (red) and the peak $\mathrm{Sm}^{2+}$ emission (blue), where the sample was exposed to X-ray irradiation continuously from $t=100 \mathrm{~s}$ to $t=7410 \mathrm{~s}$.

The current-voltage $(I-V)$ characteristic of the dosimeter configuration prior to any radiation exposure is shown in figure 5.19 (a). The data indicated that the silver contacts were Ohmic and a linear fit of the data gave $R_{\mathrm{T}} \approx 5 \times 10^{12} \Omega$, in agreement with the value obtained using the leaky capacitor model.

Following the $I-V$ characterisation $10 \mathrm{~V}$ were applied across the sample and both the RIC and RL were monitored during X-ray exposure. The complex charge carrier kinetics required to explain the time dependence of the RL (Section 5.2.5) suggested that the RIC would exhibit a similarly strong dependence on radiation dose history, where the concentrations of free electrons and holes in the conduction and valence bands, respectively, were responsible for the electric current during irradiation [177]. The RIC over 2 
hours of X-ray stimulation is shown in figure 5.19 (b). The total dose over the 2 hours was approximately $1 \mathrm{kGy}$.

Prior to irradiation and at $10 \mathrm{~V}$ the current through the sample was $2 \mathrm{pA}$. Upon X-ray stimulation, the current rapidly increased to peak at $119 \mathrm{pA}$. The current decreased after the initial spike over the next $\sim 2150 \mathrm{~s}$ to a local minimum of $30.5 \mathrm{pA}$. Subsequently, the current increased approximately linearly for the remainder of the stimulation period at a rate of $0.15 \mathrm{fA} / \mathrm{s}$. When the X-ray beam was switched off, the current dropped to a constant value of $2.9 \mathrm{pA}$.

The RIC exhibited a strong dose history dependence as expected. However, the dependence was not easily reconciled with the behaviour of the RL. The initial current spike to $119 \mathrm{pA}$ could be explained by an internal polarisation created by the electron and hole trapping properties of the system. Immediately upon X-ray exposure a large number of electrons and holes were produced in the compound that migrated through the host bands. As the charges were biased toward opposite electrical contacts, those charges that were captured in traps would exhibit a similar bias, producing a polarisation field opposing that of the external field, and consequently reducing the current from the initial peak value. Once the trap concentrations approached saturation, the polarisation field would reach a constant value and the current would reach a local minimum during the period of X-ray exposure. The decay from the peak RIC to the minimum is indicative of at least two trapping rates, on the orders of $\sim 10 \mathrm{~s}$ and $\sim 500 \mathrm{~s}$. This effect would couple with the decrease in conduction band electrons expected as the OSL-active hole traps filled during irradiation (Section 5.2.5).

The steady state leakage current increased from $2 \mathrm{pA}$ prior to irradiation to $2.9 \mathrm{pA}$ after irradiation. A similar phenomenon has been observed in diamond dosimeters [51] which was attributed to the filling of traps that, when empty, increase the resistance of the compound. This interpretation likely extends to $\mathrm{NaMgF}_{3}: \mathrm{Sm}$, where the previously discussed traps such as F-type centres and $\mathrm{Sm}^{2+} / \mathrm{Sm}^{3+}$ could affect the intrinsic resistivity of the compound.

Figures 5.19 (c) and (d) show the RL spectra and the time-dependence of the integrated RL intensities, respectively. The RL was monitored alongside the RIC. The data behaved as expected per the previous analysis of the RL (Section 5.2.5), indicating that the $10 \mathrm{~V}$ applied field did not influence the RL recombination rates significantly. After the irradiation ceased, the $\mathrm{Sm}^{2+} \mathrm{RL}$ decreased to zero immediately, whereas the $\mathrm{Sm}^{3+}$ $\mathrm{RL}$ decayed to zero over approximately 10 minutes. The appearance of an afterglow confirmed the existence of shallow, room temperature traps in the compound that were 
slowly released post-irradiation, and recombined to produce $\mathrm{Sm}^{3+}$ luminescence. The decay consisted of several components with approximate lifetimes similar to those seen in the RIC decay, suggesting that shallow traps may have caused the current decay after the initial peak.

After the first period of X-ray irradiation, the $I-V$ characterisation was repeated where the sample was actively exposed to X-ray irradiation. The X-ray photocurrent is plotted in figure 5.19 (a), where the leakage current in the absence of irradiation was subtracted from the current measured during X-ray exposure. The $S$-shaped curve implied the existence of saturation currents at high voltages, at which point all free charges would be collected by the electrodes. Such behaviour is expected when the dominant excitation process is the photoelectric effect, which was the case for the X-ray energies used in this work. The Hecht equation [178-180] was used to determine the mobilitylifetime product, $\mu \tau$, written

$$
I(V)=\frac{I_{0} \mu \tau V}{d^{2}}\left[1+\exp \left(-d^{2} / \mu \tau V\right)\right]
$$

where $I_{0}$ is the current at saturation, $V$ is the applied voltage, and $d$ is the distance between the electrodes. The data were fitted to equation 5.23 and the values $I_{0} \approx 50 \mathrm{pA}$ and $\mu \tau=1.6 \times 10^{-4} \mathrm{~cm}^{2} / \mathrm{V}$ were obtained. The mobility lifetime product was in the intermediate range for electronic dosimeters. Single crystals of some lead halide perovskites have exhibited $\mu \tau$ over two magnitudes greater than that of $\mathrm{NaMgF}_{3}: \mathrm{Sm}$ [181-183], while CVD diamond dosimeters have shown mobility-lifetime products up to two magnitudes lower than that of $\mathrm{NaMgF}_{3}: \mathrm{Sm}[180,184,185]$. The mobility-lifetime product is proportional to the magnitude of the currents produced during X-ray stimulation and therefore acts as a figure of merit for any aspiring electronic dosimeter compound. Consequently, the data indicated that $\mathrm{NaMgF}_{3}: \mathrm{Sm}$ should have a radiation sensitivity comparable to that of the recently developed CVD diamond dosimeters. It should also be noted that the RL intensities did not vary as a function of voltage.

Dose-rate characterisations were performed for the RIC and the two RL emissions, all of which were obtained simultaneously. Figure 5.20 (a) illustrates the RIC and RL magnitudes as a function of time, where the X-ray tube current was increased from 10 $\mathrm{mA}$ to $40 \mathrm{~mA}$ in $5 \mathrm{~mA}$ steps, then reduced in the same manner. Both the RIC and RL data exhibited staircase structure, where the magnitudes of each component were proportional to the dose rate. There was an initial decay observed in the RIC immediately upon $\mathrm{X}$-ray exposure that was consistent with the previously discussed data. The absence of this decay for subsequently higher dose rates was expected based on the shallow trap in- 

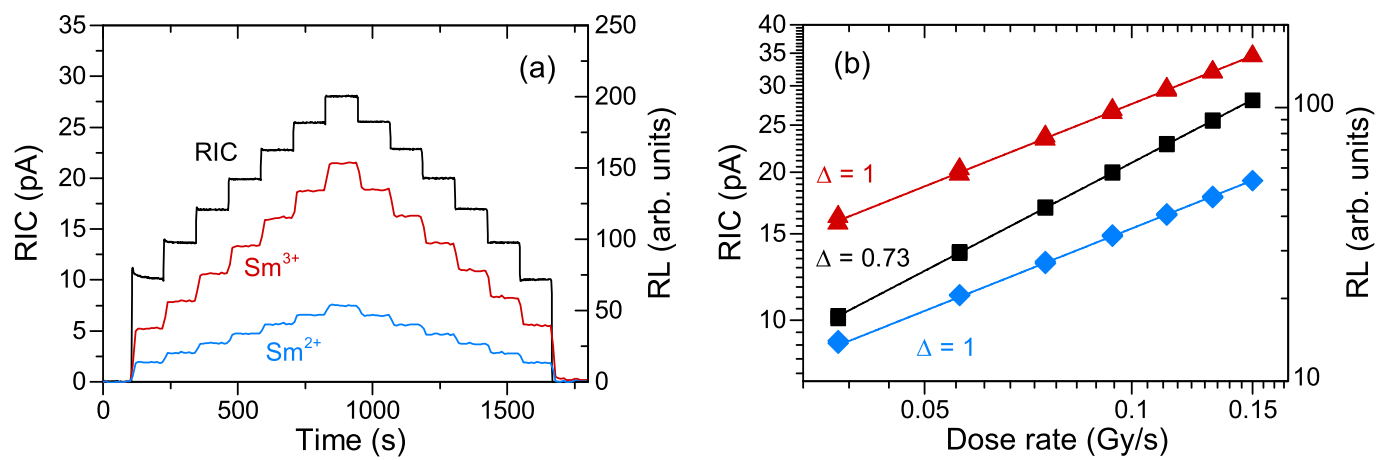

Figure 5.20: (a) RIC as a function of time, where the X-ray dose rate was varied by increasing the tube current in $5 \mathrm{~mA}$ steps then decreasing in $5 \mathrm{~mA}$ steps (black). The concurrent integrated RL intensities for $\mathrm{Sm}^{3+}$ (red) and $\mathrm{Sm}^{2+}$ (blue) are also shown. (b) RIC values (black squares) and integrated RL intensities of $\mathrm{Sm}^{3+}$ (red triangles) and $\mathrm{Sm}^{2+}$ (blue diamonds) as a function of real-time X-ray dose rate. Solid lines are fits using equation 5.24 .

terpretation, as small changes in the dose rate would not significantly affect the trapping and detrapping rates after the initial period of exposure.

The average magnitudes over the $\sim 120$ s periods of exposure at each dose rate are plotted in figure 5.20 (b). The RIC data followed a power law per the Fowler equation $[51,52]$, where

$$
i_{\mathrm{RIC}}=\gamma \dot{D}^{\Delta}
$$

Fitting the data to equation 5.24 gave an exponent of $\Delta=0.73$ for the RIC, similar to the values obtained for some CVD diamond dosimeters [52]. The RL intensities were linear with dose rate $(\Delta=1)$ as expected. The minimum detectable dose rates were approximated and were $46 \mu \mathrm{Gys}^{-1}$ using the RIC and $1.5 \mathrm{mGys}^{-1}$ using the RL.

\subsubsection{Summary and Discussion}

In Section 5.1 it was demonstrated that $\mathrm{NaMgF}_{3}: \mathrm{Sm}$ could function as an RPL dosimeter, where the X-ray induced valence conversion $\mathrm{Sm}^{3+} \rightarrow \mathrm{Sm}^{2+}$ was readily detected using PL and was proportional to the X-ray dose. The current section expanded greatly on this observation. The effects of irradiation on the $\mathrm{NaMgF}_{3}: \mathrm{Sm}$ luminescence were explored and characterised in detail.

Optical absorption measurements taken before and after irradiation revealed the presence of several F-type centres only after irradiation. Similar measurements taken 
after optical stimulation into the F-centre band clearly showed radiation-produced $\mathrm{Sm}^{2+}$, in accordance with the preliminary PL characterisation.

As the F-type centres were optically bleached, it was hypothesised that OSL should occur in the material along with the Sm RPL. This was the case, where $\mathrm{Sm}^{3+}$ was the only OSL recombination centre and F-centres were the main electron traps. The lack of any $\mathrm{Sm}^{2+}$ OSL suggested that the aliovalent substitution of $\mathrm{Sm}^{3+}$ for $\mathrm{Na}^{+}$sites encouraged the production of local defects that acted as hole traps and OSL recombination centres, where the recombination energy was transferred to the nearby $\mathrm{Sm}^{3+}$ ions. The OSL decays required three exponential components to fit, in accordance with the several Ftype centre configurations and the total bleaching of all centres when stimulated with F-centre light. The integrated OSL intensities were linear up to at least $100 \mathrm{~Gy}$ and the minimum detectable dose using the PL spectrometer for OSL stimulation and detection was $1.2 \mathrm{mGy}$.

The $\mathrm{Sm}^{3+} \rightarrow \mathrm{Sm}^{2+} \mathrm{RPL}$ was characterised as a function of dose using the ratio of the peak emissions for each valence, using equation 5.10. The data were linear up to approximately 500 Gy. All RPL measurements were non-destructive when excited at $\lambda_{\text {ex }}$ $=405 \mathrm{~nm}$ and the RPL was found to be incredibly stable, where no significant changes in the $\mathrm{Sm}^{2+} / \mathrm{Sm}^{3+}$ ratio were observed over a period of 7 weeks. Analysis of the ratios of the ${ }^{5} \mathrm{D}_{0} \rightarrow{ }^{7} \mathrm{~F}_{1}$ and ${ }^{5} \mathrm{D}_{0} \rightarrow{ }^{7} \mathrm{~F}_{2}$ emissions of $\mathrm{Sm}^{2+}$ revealed that the X-ray produced $\mathrm{Sm}^{2+}$ was more distorted than the pre-existing $\mathrm{Sm}^{2+}$. Saturation of the RPL occurred after only a small fraction of the total Sm was converted to $\mathrm{Sm}^{2+}$, and therefore only specific $\mathrm{Sm}^{3+}$ sites could trap electrons and remain stable. It was suggested that this could occur when a nearby hole trap existed nearby $\mathrm{Sm}^{3+}$, per the interpretation of the OSL process.

The $\mathrm{Sm}^{3+} \rightarrow \mathrm{Sm}^{2+}$ valence conversion was also studied using magnetisation in an attempt to quantify the concentrations of the $\mathrm{Sm}^{2+}$ and $\mathrm{Sm}^{3+}$ ions. The behaviour of the volume susceptibility of the compound was primarily paramagnetic owing to the $\mathrm{Sm}$ ions, with a diamagnetic background arising from the $\mathrm{NaMgF}_{3}$ host. This analysis suggested that $10 \%$ of the total Sm was in the divalent state prior to irradiation.

The $\mathrm{RL}$ of both the $\mathrm{Sm}^{2+}$ and $\mathrm{Sm}^{3+}$ sites was studied as a function of total X-ray dose up to high doses. The RL directly contradicted the RPL, where the $\mathrm{Sm}^{2+}$ intensity decreased with dose while the $\mathrm{Sm}^{3+}$ intensity increased. In order to understand this apparent contradiction, the RL was modeled using a charge kinetics model featuring an electron trap, a hole trap, and two recombination centres. This model was unique among RL models in that it featured an OSL-active hole trap that could capture free electrons, and that was independent of the recombination centres itself. This addition to the 
model was consistent with the observation of OSL via $\mathrm{Sm}^{3+}$ and not via $\mathrm{Sm}^{2+}$, which implied that $\mathrm{Sm}^{3+}$ locally traps the holes necessary for OSL upon F-centre electron release. The differential equations describing the model were numerically computed using MATLAB software, where several key parameters were experimentally determined using the aforementioned optical absorption, magnetisation, and luminescence data. The model was fitted to the experimental data and was quantitatively accurate, indicating that the data could be satisfactorily approximated by the relatively simple model. Furthermore, the model was entirely consistent with all other luminescence data, reinforcing its validity in the description of the complex RL system.

The RL could also be used to monitor F-type centre concentrations using the ratio of some distinct $\mathrm{Sm}^{3+}$ emissions, where the relative influence of the $\mathrm{F}_{3}$-centre absorptions on the different transitions was exploited. Additionally, it was shown that a straightforward algebraic manipulation of the $\mathrm{Sm}^{2+}$ transition intensities could be used to render the dose history dependence of the RL negligible. This suggested that the compound could be used as a real-time RL dosimeter without the need for a priming dose.

An alternative method of determining the trap depth of $\mathrm{Ln}^{2+}$ ions in host compounds was demonstrated using the thermal quenching and RPL of $\mathrm{Sm}^{2+}$. It was confirmed that the primary process by which the luminescence of $\mathrm{Sm}^{2+}$ was thermally quenched was by excitation into the conduction band and subsequent non-radiative decay to the $\mathrm{Sm}^{2+}$ ground state. This was achieved by demonstrating that the X-ray induced $\mathrm{Sm}^{2+}$ could be reconverted to $\mathrm{Sm}^{3+}$ via a photothermal ionisation process that also quenched the luminescence. This technique placed the trap depth of $\mathrm{Sm}^{2+} 4 \mathrm{eV}$ below the conduction band edge, highlighting the stability of the RPL under both optical and thermal stimulations. During this study, it was demonstrated that the RPL of $\mathrm{Sm}^{2+}$ could be monitored in real-time and was linear to at least $300 \mathrm{~Gy}$.

The thermal quenching technique allowed for the establishment of a configurational coordinate diagram for the $\mathrm{NaMgF}_{3}: \mathrm{Sm}^{2+}$ system that accurately described the effects of temperature and optical stimulation on the luminescence. Additionally, it allowed for the construction of a revised HRBE diagram where the levels of all $\mathrm{Ln}^{2+}$ and $\mathrm{Ln}^{3+}$ were placed with greater accuracy than those presented in figure 5.8. It should also be noted that the well-described temperature dependence of the $\mathrm{Sm}^{2+}$ luminescence could be used for all-optical temperature sensing in environments where wired sensors are impractical, e.g. in flammable environments.

Finally, a novel dual optical/electrical radiation sensing technique was demonstrated using the combined RIC and $\mathrm{RL}$ in $\mathrm{NaMgF}_{3}: \mathrm{Sm}$. The dosimeter configuration could be 
modeled as a leaky capacitor, where exposure to X-rays produced electron-hole pairs in the host bands that could contribute to an electrical current, and could also recombine via $\mathrm{Sm}^{3+}$ and $\mathrm{Sm}^{2+}$ ions to produce real-time luminescence. Thus, the X-ray dose rate could be monitored in real-time using the RIC and RL simultaneously, increasing the accuracy of the dosimeter and allowing for additional analyses of doses using the unique properties of the RL over time. The RIC followed a power law with an exponent $\Delta=0.73$. The minimum detectable dose rates using the RIC and RL were $46 \mu \mathrm{Gys}^{-1}$ and $1.5 \mathrm{mGys}^{-1}$, respectively, and thus were sufficiently sensitive to be applied in radiotherapy quality assurance.

In summary, $\mathrm{NaMgF}_{3}: \mathrm{Sm}$ was shown to be a strong potential candidate for radiation dose monitoring. OSL, RPL, RL, and RIC could all be exploited to measure doses, where in particular the OSL and RPL could be used in tandem for passive detection, and the RPL, RL, and RIC could be used simultaneously in real-time dose rate detection. The dose characterisations of all phenomena indicated that they could be used over wide dose ranges from approximately $1 \mathrm{mGy}$ up to several hundred Gy.

In addition to verifying the viability of the compound for radiation dose monitoring, several techniques were developed and used to model the system that could be useful in the study of a range of different compounds. Firstly, the development of an RL model that did not rely on hole trapping at the RL-active centres, but instead focused on direct recombination and hole trap at an alternative centre, described the $\mathrm{RL}$ of $\mathrm{NaMgF}_{3}: \mathrm{Sm}$ with great accuracy. This model could likely be applied to a number of other Ln-doped compounds that are known to exhibit RL and OSL. Secondly, it was shown that the high temperature thermal quenching of the $\mathrm{Sm}^{2+}$ luminescence was certainly due to photothermal ionisation of electrons to the conduction band. Consequently, establishing the activation energy of the quenching component could be used to determine the divalent Ln trap depth. This technique can be used for any Ln-doped compound that shows RPL where $\mathrm{Ln}^{3+} \rightarrow \mathrm{Ln}^{2+}$, though will be particular useful in fluoride based compounds where the $\mathrm{Ln}^{2+}$ trap depths exceed $\sim 3 \mathrm{eV}$. Finally, dual optical and electrical detection was demonstrated for the first time. This technique combines the promising aspects of CVD diamond dosimeters with that of RL dosimeters, and consequently should result in devices with greater accuracy and versatility. The experimental demonstration of the dual detection technique should encourage similar studies in alternative compounds, particularly in CVD diamonds that could potentially incorporate RL-active luminescence centres. 


\subsection{Chapter Conclusions}

The study of the luminescence of several $\mathrm{Ln}^{2+}$ and $\mathrm{Ln}^{3+}$ ions in the near tissue-equivalent $\mathrm{NaMgF}_{3}$ compound revealed properties suitable for radiation dose monitoring that provided high accuracy over large dose ranges and great versatility, as several properties could be employed simultaneously in dose monitoring. The initial PL characterisations of $\mathrm{NaMgF}_{3}: \mathrm{Ln}$ compounds where $\mathrm{Ln}=\mathrm{Sm}, \mathrm{Eu}$, Dy, or $\mathrm{Yb}$ demonstrated that the Ln ions would commonly dope in both $\mathrm{Ln}^{3+}$ and $\mathrm{Ln}^{2+}$ configurations, where the concentrations of each valence varied with $\mathrm{Ln}$ ion. $\mathrm{Eu}^{2+}$ dominated in the Eu-doped compound. For $\mathrm{Ln}$ $=\mathrm{Sm}$ and $\mathrm{Yb}$ both $\mathrm{Sm}^{2+}$ and $\mathrm{Sm}^{3+}$, and $\mathrm{Yb}^{2+}$ and $\mathrm{Yb}^{3+}$ luminescence features were comparable in intensity, and exposure to X-ray irradiation resulted in the conversions $\mathrm{Sm}^{3+}$ $\rightarrow \mathrm{Sm}^{2+}$ and $\mathrm{Yb}^{3+} \rightarrow \mathrm{Yb}^{2+}$, where the trivalent ions could trap electrons to produce their divalent counterparts. In these cases the relative changes in the $\mathrm{Ln}^{2+}$ and $\mathrm{Ln}^{3+}$ luminescence intensities were readily monitored such that the RPL could be used to measure radiation doses. For $\mathrm{Ln}=\mathrm{Dy}$, only $\mathrm{Dy}^{3+}$ was seen prior to irradiation, though $\mathrm{Dy}^{3+} \rightarrow$ $\mathrm{Dy}^{2+}$ occurred during irradiation such that the RPL of $\mathrm{Dy}^{2+}$ could be used to measure radiation doses. Additionally, as the $\mathrm{Dy}^{2+}$ ion is very uncommon, a study into the energy levels and optical transitions was performed and the results presented to provide a reference for all future studies of the ion.

For $\mathrm{Ln}=\mathrm{Sm}, \mathrm{Eu}$, and $\mathrm{Yb}, \mathrm{CTB}$ s were observed from contaminant $\mathrm{O}^{2-}$ ions. Exciting into the CTBs produced strong $4 f \rightarrow 4 f$ emissions in all cases, such that the parityforbidden aspect of the transitions could be effectively circumvented. Determining the CTB energies allowed for the construction of the first HRBE diagram for the range of $\mathrm{NaMgF}_{3}: \mathrm{Ln}$ compounds. The properties predicted by the HRBEs were in excellent agreement with all data presented in this work and those data previously published in the literature. Perhaps most importantly, this study demonstrated that $\mathrm{O}^{2-} \mathrm{CTB}$ s can be used to develop HRBEs in fluoride host compounds. Constructing HRBEs for fluoride hosts is traditionally difficult due to the high energies required to induce $\mathrm{F}^{-} \rightarrow \mathrm{Ln}^{3+}$ charge transfer. Thus, this chapter presented a new method by which one can analyse the Ln levels in fluoride hosts, where small concentrations of oxygen impurities could be intentionally introduced to the host lattice. Furthermore, the HRBE diagram for $\mathrm{NaMgF}_{3}: \mathrm{Ln}$ will serve as a valuable reference in all future studies of the $\mathrm{NaMgF}_{3}: \mathrm{Ln}$ compounds, as all $\mathrm{Ln}^{2+/ 3+}$ trap depths and $5 d$ levels, relative to the other ions and the host bands of $\mathrm{NaMgF}_{3}$, are contained in the diagram. Knowledge of these properties allows one to predict the RPL and OSL properties of a compound and also to investigate the local structures of ions 
based on their perturbations from the predicted values.

$\mathrm{NaMgF}_{3}: \mathrm{Sm}$ was selected as a promising $\mathrm{NaMgF}_{3}: \mathrm{Ln}$ compound for dosimeter applications due to its well-defined luminescence from $\mathrm{Sm}^{3+}$ and $\mathrm{Sm}^{2+}$ in the region $500 \mathrm{~nm}$ - $800 \mathrm{~nm}$ that could be probed non-destructively at an accessible wavelength $\left(\lambda_{\mathrm{ex}}=405\right.$ $\mathrm{nm})$. The compound was studied in detail, including via optical absorption, magnetisation, various luminescence phenomena, and electrical conduction.

OSL was observed post-irradiation, where the electron traps involved were F-type centres that could be completely bleached using F-centre light near $300 \mathrm{~nm}$. The OSL emission occurred only through $\mathrm{Sm}^{3+}$, implying the existence of localised hole traps at or nearby the $\mathrm{Sm}^{3+}$ ions that served as OSL recombination centres. The OSL could be used to monitor radiation doses up to at least $100 \mathrm{~Gy}$. RPL was observed as X-ray induced $\mathrm{Sm}^{3+} \rightarrow \mathrm{Sm}^{2+}$ conversion, and the ratio of the PL emissions of each valence could be used to monitor doses up to approximately $500 \mathrm{~Gy}$. OSL stimulation did not bleach the RPL due to the large trap depth of the $\mathrm{Sm}^{2+}$ ion, such that individual doses could be monitored destructively via OSL, and cumulative doses monitored non-destructively via RPL. The compound could be entirely optically reset upon an extended $254 \mathrm{~nm}$ bleach. Thus, the compound was first demonstrated to have potential applications as a hybrid OSL/RPL dosimeter.

The RL of the compound was unusual in that the behaviour of the $\mathrm{Sm}^{2+}$ and $\mathrm{Sm}^{3+}$ RL emissions directly contrasted the behaviour the RPL. An RL model was developed to explain this effect, consisting of individual electron and hole traps and two recombination centres corresponding to the two Sm valences. While the model certainly simplified the true number of electron and hole traps, the equations detailing the charge kinetics upon irradiation were numerically computed and found to be in excellent agreement with the experimental data. The major difference between the model presented in this chapter and historic RL models is that the recombination centres were not considered to be hole traps, but instead straightforward recombination centres, where simultaneous electron-hole recombination produced RL emissions that were constant in time. The time dependence then resulted from the presence of the hole trap considered to exist nearby $\mathrm{Sm}^{3+}$ and that produced OSL after irradiation. As free electrons could combine with the hole trap, the total emissions via $\mathrm{Sm}^{3+}$ increased over time, while those of $\mathrm{Sm}^{2+}$ necessarily decreased as the concentration of available recombination electrons was reduced. This model should be useful in describing the RL of any Ln ion in any OSL-active material and thus should find applications elsewhere, in addition to providing valuable insight into the charge kinetics of the $\mathrm{NaMgF}_{3}: \mathrm{Sm}$ system. 
The thermal quenching of the $\mathrm{Sm}^{2+}$ ion was studied in an attempt to obtain an accurate measurement of the $\mathrm{Sm}^{2+}$ trap depth. By demonstrating that X-ray produced $\mathrm{Sm}^{2+}$ could be reconverted to $\mathrm{Sm}^{3+}$ upon photothermal stimulation, it was verified that the mechanism of quenching was the photothermal ionisation of electrons from the $\mathrm{Sm}^{2+}$ ion to the conduction band. This analysis was necessary in the construction of an accurate HRBE diagram and should be of use in future studies of fluoride based compounds, as the $\mathrm{Ln}^{2+}$ trap depths are difficult to measure using standard TL techniques.

Finally, it was shown that $\mathrm{NaMgF}_{3}: \mathrm{Sm}$ can also operate as an electric dosimeter, where a measurable RIC was produced during irradiation when a voltage was applied. It was shown that the configuration functioned as a leaky capacitor in the absence of irradiation, and upon X-ray stimulation electron-hole pairs produced a RIC. The applied voltage did not affect the RL emissions, such that the RL and RIC could be monitored simultaneously. As such, it was demonstrated that $\mathrm{NaMgF}_{3}: \mathrm{Sm}$ may also function as a hybrid optical/electrical dosimeter using RL/RICs that could be detected in real-time.

In summary, the $\mathrm{NaMgF}_{3}$ :Ln compounds were shown to exhibit luminescence features suitable for radiation monitoring. In particular, $\mathrm{NaMgF}_{3}: \mathrm{Sm}$ was shown to be incredibly versatile, where the RPL and OSL could be used for passive dose detection, and the RPL, RL, and RICs could be used for active dose detection. The various luminescence features of the compound were modeled in several ways, improving our understanding of the compound, and providing bases for future studies of similar compounds. Of particular note are the novel RL model, photothermal ionisation technique, and dual electrical/optical detection methods described in this chapter, all of which should encourage similar studies on other promising dosimeter compounds. 



\section{Chapter 6}

\section{Development of a Two-dimensional Dosimeter System}

In order to better demonstrate the usefulness of the materials studied herein as dosimeters, a two-dimensional (2D) dosimeter system was developed. The system functioned as a working prototype suitable for radiotherapy dose verification in the case of the $\mathrm{NaMgF}_{3}$ materials, though could also be used for X-ray imaging. This chapter will introduce the simple system used for initial testing, describe the results of initial testing, and discuss the development of the system. The means of dose detection in all cases was OSL, and the compounds tested were $\mathrm{NaMgF}_{3}: \mathrm{Mn}$ and $\mathrm{NaMgF}_{3}: \mathrm{Eu}$.

\subsection{Description of the Initial System}

The previous Chapters 4 and 5 detailed multiple techniques through which radiation doses could be monitored using the luminescence of doped fluoroperovskites. The majority of samples showed OSL after irradiation, where optical stimulation of the sample resulted in a detectable luminescence with intensity proportional to X-ray dose. Several samples showed RPL, where changes in the photoluminescence were detected before and after irradiation. The $2 \mathrm{D}$ system was initially designed to operate using passive detection techniques such as OSL and RPL. The material of interest was externally irradiated then transferred to the readout device, whereupon it was optically stimulated and the emission studied as a function of X-ray dose.

The initial design of the $2 \mathrm{D}$ readout system is shown in figure 6.1. Optical stimulation was achieved using a single high intensity LED capable of stimulating the OSL emissions with reasonable intensities, and capable of entirely bleaching the sample within a few 


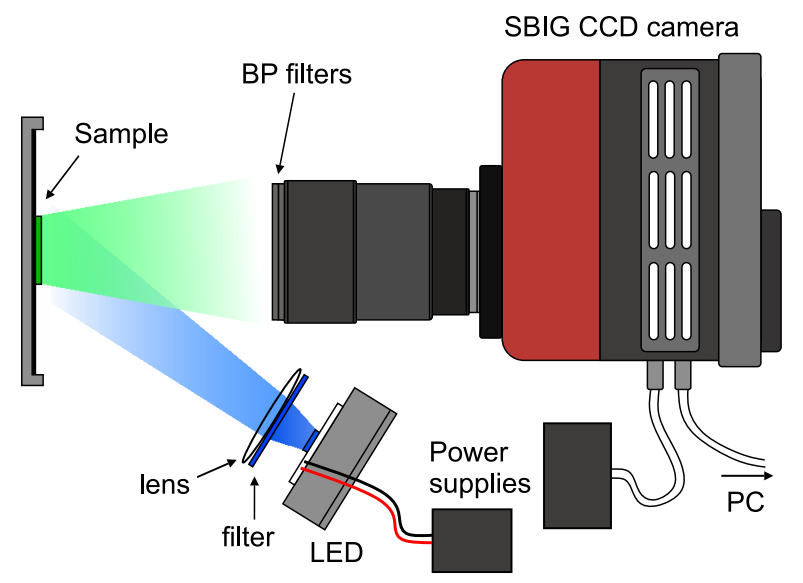

Figure 6.1: Schematic illustration of the initial 2D dosimeter prototype. The light from an LED passed through an optical filter and a focusing lens before reaching the irradiated sample. The OSL/RPL from the sample was emitted and passed through a bandpass filter before being detected by the CCD camera. The produced images were converted to .tiff format via a PC connection.

hours. When necessary, optical filters were employed between the LED and the sample and/or the sample and the detecting camera. This prevented detection of the stimulation light and improved the fraction of detected light that arose from OSL processes. Similarly, focusing lenses were employed in some cases to improve the stimulation intensity and uniformity.

The most important component of the readout system was the detector. A detector suitable for 2D dose mapping required good spectral sensitivity, high resolution, and rapid readout. The detector employed in the readout system was a SBIG STXL-11002 monochrome CCD camera that is more commonly used for astronomical imaging. The camera consisted of an $11.1 \mathrm{mp}$ KAI-11002 CCD detector with a quantum efficiency of $50 \%$ and a $9 \mu \mathrm{m}$ pixel size. It also featured an active thermoelectric cooling system that could reduce the temperature of the $\mathrm{CCD}$ down to $-60^{\circ} \mathrm{C}$ below ambient temperature. A Canon EF $35 \mathrm{~mm} \mathrm{f} / 1.4$ lens was used for image focusing, and some optical bandpass filters were attached to the lens where appropriate. Ultimately, images were converted to .tiff format via a 16 bit ADC and exported for further analysis.

Initial characterisations of $\mathrm{NaMgF}_{3}: \mathrm{Eu}$ and $\mathrm{NaMgF}_{3}: \mathrm{Mn}$ were made using the initial design as described above. Due to the unique OSL stimulation and emission properties of each sample, the LEDs and optical filters employed in each characterisation were different. For all 2D measurements discussed throughout the remainder of this chapter, the camera CCD was cooled to $-20^{\circ} \mathrm{C}$. 


\subsection{D Dosimetry with $\mathrm{NaMgF}_{3}: \mathrm{Eu}$}

The first compound to be characterised using the $2 \mathrm{D}$ dosimeter system was $\mathrm{NaMgF}_{3}: \mathrm{Eu}$. The compound has been shown to exhibit OSL post-irradiation that can be used to monitor radiation doses [28]. The OSL emission centre is $\mathrm{Eu}^{2+}$ with a peak emission at $360 \mathrm{~nm}$ [28]. The integrated OSL is linear over a very wide dose range, from $\sim 1 \mu \mathrm{Gy}$ to $\sim 100 \mathrm{~Gy}$ [28]. These properties encouraged a comprehensive characterisation of the compound regarding potential $2 \mathrm{D}$ dosimeter applications.

\subsubsection{Initial Characterisation}

The basic OSL properties of $\mathrm{NaMgF}_{3}: 0.15 \%$ Eu were characterised in one-dimension using the spectrofluorometer prior to the characterisation using the 2D system. Figure 6.2 (a) illustrates the basic PL properties of $\mathrm{NaMgF}_{3}$ :Eu with emphasis on the $\mathrm{Eu}^{2+}$ ion. The PL excitation spectrum where $\lambda_{\mathrm{em}}=359 \mathrm{~nm}$ showed a broad curve characteristic of several overlapping $4 f \rightarrow 5 d$ absorptions, as expected for the divalent $\mathrm{Ln}^{2+}$. Exciting into the peak of the $4 f^{6} 5 d^{1}$ band at $\lambda_{\text {ex }}=275 \mathrm{~nm}$ produced a line emission that peaked at $359 \mathrm{~nm}$ that was due to the zero phonon ${ }^{6} \mathrm{P}_{7 / 2} \rightarrow{ }^{8} \mathrm{~S}_{7 / 2}$ transition within the $4 f^{7}$ configuration of $\mathrm{Eu}^{2+}$. The broad band beneath the line emission that peaked about $375 \mathrm{~nm}$ was due to $5 d \rightarrow 4 f$ emission of $\mathrm{Eu}^{2+}$. The strong ${ }^{6} \mathrm{P}_{7 / 2} \rightarrow{ }^{8} \mathrm{~S}_{7 / 2}$ emission relative to the $5 d \rightarrow 4 f$ emission occurred due to the small crystal field splitting of the $5 d$ levels in the $\mathrm{NaMgF}_{3}$ host and efficient energy transfer from the $5 d$ level to the ${ }^{6} \mathrm{P}_{7 / 2}$ level [28].

The basic OSL properties of the compound are shown in figure $6.2(\mathrm{~b})$, where in this case the stimulation wavelength $\left(\lambda_{\mathrm{ex}}=445 \mathrm{~nm}\right)$ was longer than the emission wavelength $\left(\lambda_{\mathrm{em}}=359 \mathrm{~nm}\right)$ such that no PL emission would typically occur. No PL was observed prior to irradiation. After an X-ray dose of $10 \mathrm{~Gy}$ and when exciting at $\lambda_{\mathrm{ex}}=445 \mathrm{~nm}$, the ${ }^{6} \mathrm{P}_{7 / 2} \rightarrow{ }^{8} \mathrm{~S}_{7 / 2}$ and $5 d \rightarrow 4 f$ emissions of $\mathrm{Eu}^{2+}$ were observed. The excitation spectrum where $\lambda_{\mathrm{em}}=359 \mathrm{~nm}$ taken after a 10 Gy X-ray dose showed a broad contribution that peaked below $400 \mathrm{~nm}$ and extended out to approximately $550 \mathrm{~nm}$. This behaviour was representative of an OSL process, where the longer wavelength excitation energy was insufficient in exciting the $\mathrm{Eu}^{2+}$ ion directly, but was capable of releasing charges stored in the various trapping sites in the compound. The released charges recombined with trapped holes and the energy was transferred to the $\mathrm{Eu}^{2+}$ ions, producing the characteristic luminescence. The OSL stimulation curve correlated with the F-centre absorptions seen in $\mathrm{NaMgF}_{3}: \mathrm{Sm}$ and $\mathrm{NaMgF}_{3}: \mathrm{Mn}$ that peaked near $290 \mathrm{~nm}$ and extended out to approximately $500 \mathrm{~nm}$, such that the OSL-active traps were attributed to F-centres. The 
OSL-active hole traps were likely localised to $\mathrm{Eu}^{2+}$ due to the charge compensation required for the substitution $\mathrm{Eu}^{2+} \longleftrightarrow \mathrm{Na}^{+}$. It is also possible that $\mathrm{Eu}^{2+}$ itself acted as a hole trap such that $\mathrm{Eu}^{2+}+\mathrm{h}^{+} \rightarrow \mathrm{Eu}^{3+}$, as seen in other fluoroperovskites $[62,186]$.
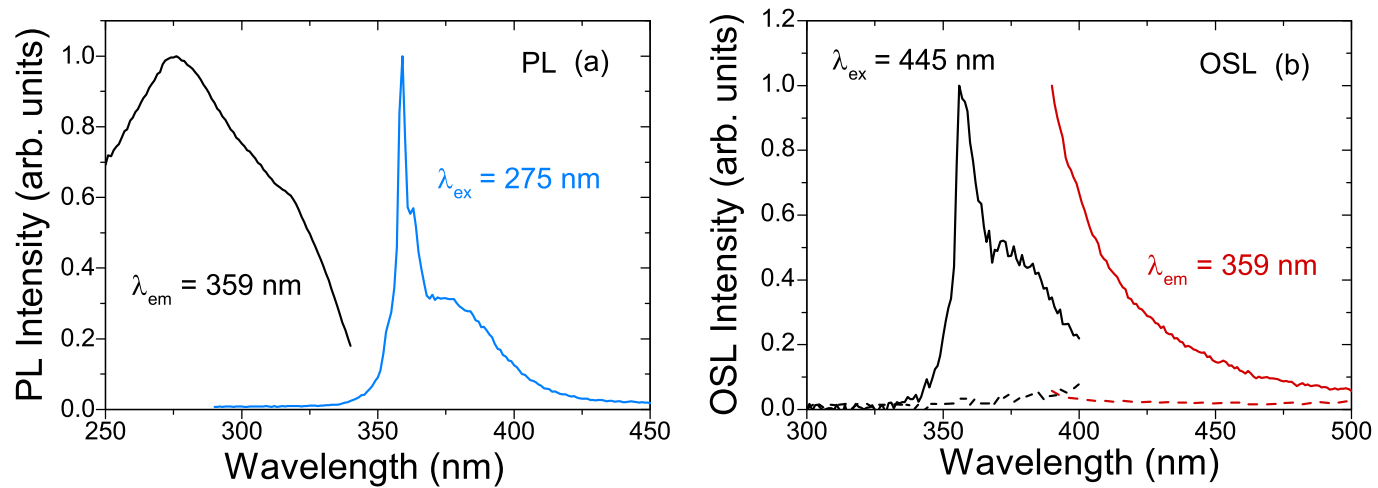

Figure 6.2: (a) PL spectra of $\mathrm{NaMgF}_{3}$ :Eu prior to irradiation where $\lambda_{\mathrm{em}}=359 \mathrm{~nm}$ (black) and $\lambda_{\text {ex }}=275 \mathrm{~nm}$ (blue). (b) OSL spectra of $\mathrm{NaMgF}_{3}:$ Eu before (dashed) and after (solid) an X-ray dose of $10 \mathrm{~Gy}$, where $\lambda_{\mathrm{ex}}=445 \mathrm{~nm}$ (black) and $\lambda_{\mathrm{em}}=359 \mathrm{~nm}$ (red).

Figures 6.3 illustrate the OSL decay characteristics of $\mathrm{NaMgF}_{3}: \mathrm{Eu}$. With reference to the previously discussed OSL stimulation spectrum, the OSL could be stimulated using a range of wavelengths from approximately $400 \mathrm{~nm}$ to $550 \mathrm{~nm}$. It was of interest to test the linearity and sensitivity for different stimulation energies. The wavelengths $405 \mathrm{~nm}$ and $445 \mathrm{~nm}$ were selected for this purpose as they are commonly encountered LED wavelengths. The OSL decays for X-ray doses ranging from $0.5 \mathrm{~Gy}$ to $120 \mathrm{~Gy}$ are shown in figure 6.3 (a) for the stimulation $\lambda_{\mathrm{ex}}=405 \mathrm{~nm}$ and where the OSL emission was detected at the $\mathrm{Eu}^{2+}$ peak at $\lambda_{\mathrm{em}}=359 \mathrm{~nm}$. Per the $\mathrm{NaMgF}_{3}: \mathrm{Mn}$ and $\mathrm{NaMgF}_{3}: \mathrm{Sm}$ samples (Sections 4.3.4 and 5.2.3), the OSL decays could be fitted to a sum of three-exponential decays (equation 4.4). The decay constants, $\tau_{i}$, were fixed for all X-ray doses and were $\tau_{1}=3.9 \mathrm{~s}, \tau_{2}=43 \mathrm{~s}$, and $\tau_{3}=250 \mathrm{~s}$. The use of three components to fit the decays concurs with those data presented for other doped $\mathrm{NaMgF}_{3}$ compounds, where multiple components are required to due to the contributions from the several F-type centres that exist in the $\mathrm{NaMgF}_{3}$ host lattice [130].

The integrated OSL intensities were calculated from the fits as $A_{\mathrm{OSL}}(D)=\sum_{i} A_{i}(D) \tau_{i}$ and are plotted in figure 6.3 (b). The dose response was linear up to approximately $20 \mathrm{~Gy}$, after which $A_{\mathrm{OSL}}(D)$ tended to sub-linearity. The noise equivalent dose was calculated using the errors in the fitted $A_{\mathrm{OSL}}(D)$, defined as the dose at which $A_{\mathrm{OSL}}(D)$ is equivalent to the uncertainty in $A_{\mathrm{OSL}}(D)$. For $\lambda_{\mathrm{ex}}=405 \mathrm{~nm}$, the noise equivalent dose was calculated to be $2.9 \mathrm{mGy}$. 

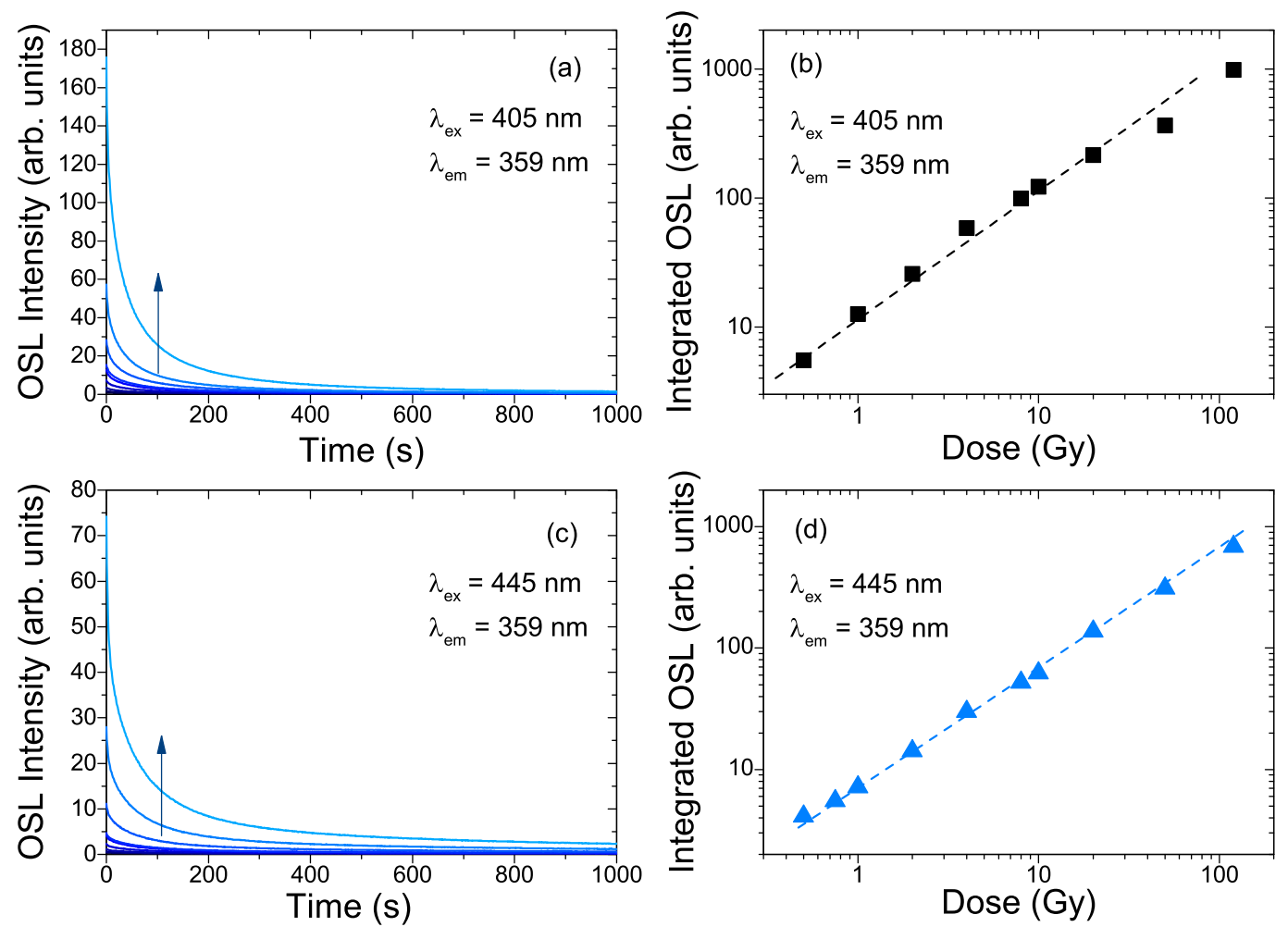

Figure 6.3: (a) OSL decay curves for $\mathrm{NaMgF}_{3}$ :Eu obtained after X-ray doses ranging from $0.5 \mathrm{~Gy}$ to $120 \mathrm{~Gy}$, where $\lambda_{\mathrm{ex}}=405 \mathrm{~nm}$ and $\lambda_{\mathrm{em}}=359 \mathrm{~nm}$. The arrow indicates the trend for higher doses. (b) Dose dependence of the integrated OSL emission intensity where $\lambda_{\mathrm{ex}}=$ $405 \mathrm{~nm}$ and $\lambda_{\mathrm{em}}=359 \mathrm{~nm}$ as determined via the fitting of the decay curves. The dashed line is a linear fit to the data. (c) OSL decay curves obtained after X-ray doses ranging from $0.5 \mathrm{~Gy}$ to $120 \mathrm{~Gy}$, where $\lambda_{\mathrm{ex}}=445 \mathrm{~nm}$ and $\lambda_{\mathrm{em}}=359 \mathrm{~nm}$. The arrow indicates the trend for higher doses. (b) Dose dependence of the integrated OSL emission intensity where $\lambda_{\mathrm{ex}}=445 \mathrm{~nm}$ and $\lambda_{\mathrm{em}}=359 \mathrm{~nm}$ as determined via the fitting of the decay curves. The dashed line is a linear fit to the data. 
The OSL decays where $\lambda_{\mathrm{ex}}=445 \mathrm{~nm}$ and $\lambda_{\mathrm{em}}=359 \mathrm{~nm}$ are shown in figure 6.3 (c). The data were fitted to the three-exponential decay function (equation 4.4) and the decay constants were $\tau_{1}=4.1 \mathrm{~s}, \tau_{2}=68 \mathrm{~s}$, and $\tau_{3}=590 \mathrm{~s}$. The OSL emission intensities decreased relative to those encountered under $405 \mathrm{~nm}$ stimulation for the same X-ray doses in accordance with the OSL stimulation spectrum, where the peak stimulation wavelength was near $400 \mathrm{~nm}$. This suggested that the absorption cross-sections of the various F-type centres that produced the OSL were lower at longer wavelengths. This property also had the effect of increasing the decay constants under $445 \mathrm{~nm}$ stimulation relative to $405 \mathrm{~nm}$ stimulation.

The integrated OSL intensities $A_{\mathrm{OSL}}(D)$ for $\lambda_{\mathrm{ex}}=445 \mathrm{~nm}$ are plotted in figure 6.3 (d). The dose response was linear up to at least $50 \mathrm{~Gy}$, indicating that lower energy stimulations could be used to expand the working dose range of the OSL dosimeter. However, the noise equivalent dose was calculated to be $5.8 \mathrm{mGy}$ in this case, suggesting that any increase in the linear response range would be coupled with a loss in sensitivity.

It should be noted that a previous study has shown that doses on the order of $\sim 100$ nGy could be detected using the OSL of $\mathrm{NaMgF}_{3}: \mathrm{Eu}$ using alternative detector optics [28]. Consequently, the limitations on the working dose range were largely driven by the spectrofluorometer.

Finally, a sample was irradiated and subsequently bleached using $445 \mathrm{~nm}$ light and an OSL readout performed. Following this, the sample was stimulated with light corresponding to the peak of the F-centre absorption band $(280 \mathrm{~nm})$. No residual OSL was detected during the $280 \mathrm{~nm}$ stimulation, demonstrating that the samples were successfully optically reset using $445 \mathrm{~nm}$ readout.

\subsubsection{D Characterisation}

A bulk sample of $\mathrm{NaMgF}_{3}: 0.1 \% \mathrm{Eu}$ was characterised using the $2 \mathrm{D}$ system described in figure 6.1. The LED was a Luxeon Star LED operated at $400 \mathrm{~mA}$ and with peak emission intensity at $448 \mathrm{~nm}$. In order to reduce the intensity of the LED emissions at shorter wavelengths the stimulating light was transmitted through two long pass filters, with cut-off wavelengths at $420 \mathrm{~nm}$ and $435 \mathrm{~nm}$. The transmitted light was focused over the sample using an OptoSigma convex lens. The SBIG CCD camera lens was outfitted with two BP365-72 (365 nm) bandpass filters in order to ensure only the $\mathrm{Eu}^{2+}$ OSL emissions were detected. The intensity of the LED light at the sample was measured using a Thorlabs PDA100A Si detector and was approximately $0.55 \mathrm{~mW} / \mathrm{cm}^{2}$.

The OSL decay curve as collected by the CCD camera is shown in figure 6.4 (a) after 

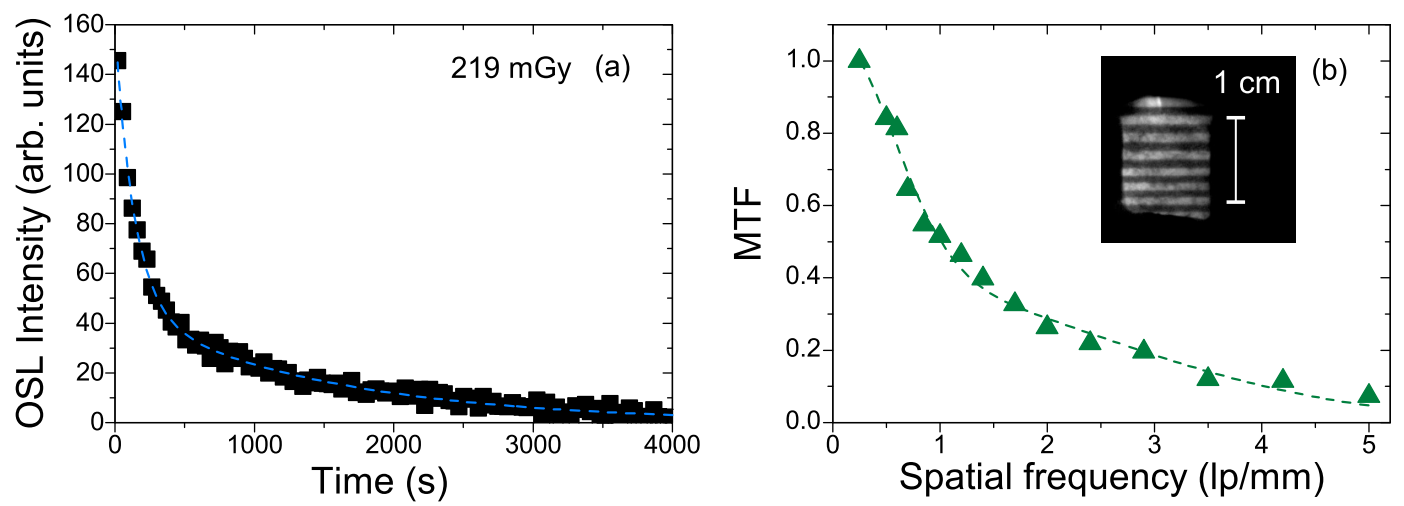

Figure 6.4: (a) 2D OSL decay of $\mathrm{NaMgF}_{3}$ :Eu where $\lambda_{\mathrm{ex}}=448 \mathrm{~nm}$ and the OSL emission was detected using the CCD camera. The X-ray dose was $219 \mathrm{mGy}$ and the dashed line is a two-exponential fit to the data. (b) MTF of bulk $\mathrm{NaMgF}_{3}$ :Eu using the CCD camera for OSL detection. The dashed line is a guide to the eye. Inset: X-ray image of the sample when masked by the $\mathrm{Pb}$ phantom.

an X-ray dose of approximately $219 \mathrm{mGy}$. The $448 \mathrm{~nm}$ stimulation was continuous and the images were collected using an exposure time of $30 \mathrm{~s}$. As such, the data in the figure correspond to integrated OSL intensities over the $30 \mathrm{~s}$ collection period. The decay could be fitted to the sum of two exponential decays,

$$
I_{\mathrm{OSL}}(t)=A_{1} \times \exp \left(-t / \tau_{1}\right)+A_{2} \times \exp \left(-t / \tau_{2}\right),
$$

and the decay constants were $\tau_{1}=140 \mathrm{~s}$ and $\tau_{2}=1480 \mathrm{~s}$. OSL decay constants are proportional to the absorption cross-sections of the OSL-active defects (in this case F-type centres) at the stimulation wavelengths of interest, and also the intensity of the stimulating light. As the LED emission spectrum was significantly broader than those stimulations used in the spectrofluorometer characterisations, and the LED also exhibited a different stimulation intensity, the variations in the decay constants relative to the previous characterisations were expected. The lack of a short decay constant was likely related to the fact that the OSL intensities were integrated over $30 \mathrm{~s}$, such that the rapid decay component could not be measured. The noise equivalent dose was calculated to be $14 \mathrm{mGy}$ using this setup. The stimulation efficiency, defined as the energy required to deplete the total OSL energy to $1 / \mathrm{e}$ of its initial value, was calculated to be $5.9 \mathrm{~mJ} / \mathrm{mm}^{2}$ for the $448 \mathrm{~nm}$ stimulation.

In order to assess the spatial resolution of the system, a X-ray test grating was used to mask the sample during irradiation and was removed prior to OSL readout. The grating was a Nuclear Associates X-ray Test Pattern phantom composed of $50 \mu \mathrm{m}$ thick $\mathrm{Pb}$ lines 
with spacings from $0.25 \mathrm{lp} / \mathrm{mm}$ to $10 \mathrm{lp} / \mathrm{mm}$. The sample was irradiated with high doses of approximately $44 \mathrm{~Gy}$. The X-ray image obtained from the first $30 \mathrm{~s}$ exposure in each case was used to estimate the modulation transfer function (MTF) of the system, and the MTF is plotted in figure 6.4 (b) normalised to the value at $0.25 \mathrm{lp} / \mathrm{mm}$. A standard figure of merit used to assess $2 \mathrm{D}$ systems is the spatial frequency at MTF $=0.2$, equivalent to $2.9 \mathrm{lp} / \mathrm{mm}$ in this system. The readout configuration was therefore capable of resolving sub-mm variations in X-ray dose.

\subsubsection{Summary and Conclusions}

The initial testing of the 2D dosimeter system using the OSL of $\mathrm{NaMgF}_{3}$ :Eu was promising. $\mathrm{NaMgF}_{3}: \mathrm{Eu}$ has the useful property where the OSL can be stimulated with wavelengths longer than the OSL emission wavelength, which acts to reduce any background PL signal and allows for efficient and effective optical filtering of the stimulation light. The OSL of the sample was shown to be linear up to at least $50 \mathrm{~Gy}$ during $445 \mathrm{~nm}$ stimulation, exceeding the lower limit of $\sim 10 \mathrm{~Gy}$ required for radiotherapy applications. The 2D system could theoretically monitor dose variations down to $14 \mathrm{mGy}$, approaching the required $10 \mathrm{mGy}$ sensitivity. Furthermore, the system was capable of resolving the OSL of a bulk sample down to at least $2.9 \mathrm{lp} / \mathrm{mm}$, below the encouraged $1 \mathrm{~mm}$ threshold on resolution. As such, it was demonstrated that near tissue-equivalent $2 \mathrm{D}$ dosimetry was possible using the OSL of fluoroperovskites.

The 2D monitoring could be improved via both optimisation of the sample and optimisation of the system. The latter will be addressed in Section 6.4, though it should be noted that a straightforward means of improving the minimum detectable dose would be to shift the stimulation wavelength to higher energies, e.g. $405 \mathrm{~nm}$. However, this would require higher quality optical filtering, as a majority of $405 \mathrm{~nm}$ LEDs have nonnegligible emission components at wavelengths approaching the $\mathrm{Eu}^{2+}$ peak. Thus, reflections of the stimulation light would likely be detected by the camera, increasing the image noise. The resolution of the system was largely limited by internal scattering and reflection of the OSL emissions within the $\mathrm{NaMgF}_{3}$ :Eu crystal. Decreasing the sample thickness would reduce these effects and improve the limiting resolution. Additionally, improving the homogeneity of the samples should improve the limiting resolution. Variations in crystallite size throughout the sample produced variations in the OSL emission intensities that increased the difficulty with which doses could be accurately resolved. 


\subsection{D Dosimetry with $\mathrm{NaMgF}_{3}: \mathrm{Mn}$}

The successful demonstration of the $2 \mathrm{D}$ system using $\mathrm{NaMgF}_{3}: \mathrm{Eu}$ motivated a subsequent study using the OSL of $\mathrm{NaMgF}_{3}: \mathrm{Mn}$. The radiation sensing properties of the compound are discussed in detail in Chapter 4 . The previous section also demonstrated that bulk crystalline samples suffer from inhomogeneity. This encouraged the development of films with improved uniformity.

A film was produced by grinding a polycrystalline sample of $\mathrm{NaMgF}_{3}: 0.4 \% \mathrm{Mn}$ into a fine powder using a mortar and pestle. The powder was subsequently mixed with a solution of Epo-Tek 301 epoxy resin. The volume fraction of $\mathrm{NaMgF}_{3}: \mathrm{Mn}$ was $35 \%$. The solution was cured into a rectangular film with dimensions $21.3 \mathrm{~mm} \times 27.3 \mathrm{~mm} \times 1 \mathrm{~mm}$. The film was characterised using the $2 \mathrm{D}$ system.

For the following characterisation, all X-ray irradiations were performed using the Faxitron X-ray source, operated at $35 \mathrm{kVp}$ and $0.3 \mathrm{~mA}$, where the dose rate was approximately $0.4 \mathrm{mGy} / \mathrm{s}$. The Faxitron could accurately deliver lower doses than the Phillips $\mathrm{X}$-ray generator and also delivered a more uniform dose across the sample. The LED used to stimulate the sample was a Thorlabs M365D2 LED with a peak emission intensity at $365 \mathrm{~nm}$. The LED intensity at the sample was measured to be $0.34 \mathrm{~mW} / \mathrm{cm}^{2}$. No optical filtering was used to narrow the broad stimulation band in this case. The LED stimulation area was sufficiently broad such that no focusing lens was required. The Canon lens was outfitted with two BP590-72 (590 nm) bandpass filters, where the 590 $\mathrm{nm}$ bandpass corresponded to the peak of the non-distorted $\mathrm{Mn}^{2+}$ OSL emission. The camera exposure time for all images was $2 \mathrm{~s}$.

\subsubsection{Initial Characterisation}

The $\mathrm{NaMgF}_{3}: \mathrm{Mn} /$ epoxy composite was initially characterised via PL. Figure 6.5 (a) shows the PL excitation spectrum where $\lambda_{\mathrm{em}}=600 \mathrm{~nm}$, corresponding to the non-distorted $\mathrm{Mn}^{2+}$ emission of $\mathrm{NaMgF}_{3}: \mathrm{Mn}$. The intraconfigurational $3 d$ transitions were clear, where the peak excitation from the ${ }^{6} A_{1}(S)$ ground state to the ${ }^{4} A_{1}(G)$ and ${ }^{4} E(G)$ excited states occurred at $395 \mathrm{~nm}$. In contrast to the PL of the bulk sample there was a broad background component to the excitation, indicating that the epoxy also showed PL that could negatively affect the 2D dose measurements. The LED used for OSL stimulation was 365 $\mathrm{nm}$, selected in accordance with the OSL excitation spectrum (figure 4.19 (a)) and the availability of high intensity LEDs in the UV spectral region. The PL emission spectrum where $\lambda_{\mathrm{ex}}=365 \mathrm{~nm}$ of the composite film is shown in figure 6.5 (b) prior to any irra- 

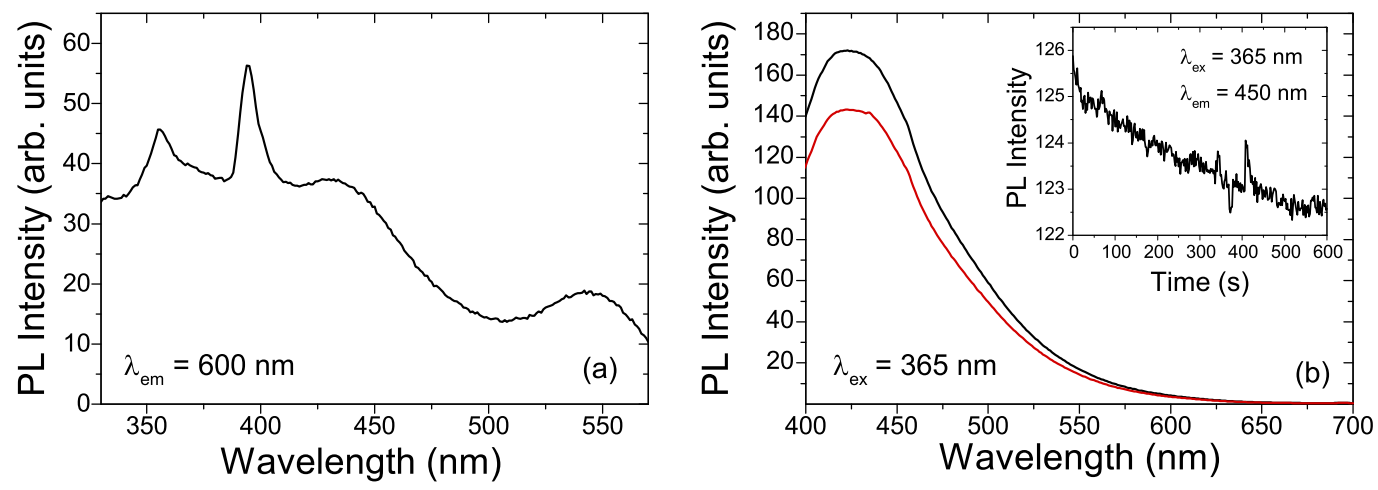

Figure 6.5: (a) PL excitation of the $\mathrm{NaMgF}_{3}: \mathrm{Mn} / \mathrm{epoxy}$ composite where $\lambda_{\mathrm{em}}=600 \mathrm{~nm}$. (b) PL emission of the composite where $\lambda_{\mathrm{ex}}=365 \mathrm{~nm}$ (black), and where $\lambda_{\mathrm{ex}}=365 \mathrm{~nm}$ after 10 minutes of $365 \mathrm{~nm}$ stimulation (red). Inset: PL decay where $\lambda_{\mathrm{ex}}=365 \mathrm{~nm}, \lambda_{\mathrm{em}}=$ $450 \mathrm{~nm}$.

diation. A broad emission peaking at approximately $425 \mathrm{~nm}$ was observed. This peak did not correspond to any expected emission from $\mathrm{NaMgF}_{3}: \mathrm{Mn}$ and was therefore attributed to the epoxy, where the excitation wavelength $365 \mathrm{~nm}$ concurs with the broad background seen in the excitation spectrum. An additional weak emission component could be seen near $500 \mathrm{~nm}$ that was due to emission from the distorted $\mathrm{Mn}^{2+}$ site. The lack of any intense non-distorted $\mathrm{Mn}^{2+}$ emissions was promising for OSL dosimeter applications, as the residual PL intensity at the wavelength of interest (590 nm) was small.

The Epo-Tek 301 resin contained bisphanol-A diglycidyl ether that, when mixed with various hardening agents, is known to fluoresce between $400 \mathrm{~nm}$ and $450 \mathrm{~nm}$ under UV stimulation $[187,188]$. It was of interest to test the temporal stability of the epoxy fluorescence due to the continuous stimulation used for 2D OSL measurements. The inset to figure 6.5 (b) shows the PL intensity at $450 \mathrm{~nm}$ under continuous $365 \mathrm{~nm}$ stimulation over 10 minutes and the PL intensity decayed over time. Figure 6.5 (b) also shows the PL emission spectrum after the $365 \mathrm{~nm}$ stimulation, where the peak intensity at $425 \mathrm{~nm}$ decreased by approximately $15 \%$. This decrease was consistent across the entire spectrum, such that a $15 \%$ decrease in emission intensity was observed at the OSL emission wavelength of $590 \mathrm{~nm}$. Thus, in order to perform accurate OSL measurements, the timedependence of the epoxy luminescence had to be collected and corrected for.

\subsubsection{D Characterisation}

The inset to figure 6.6 (a) illustrates the means by which the temporal variations of both the OSL and PL were simultaneously monitored. A piece of $1 \mathrm{~mm}$ thick $\mathrm{Pb}$ was placed 

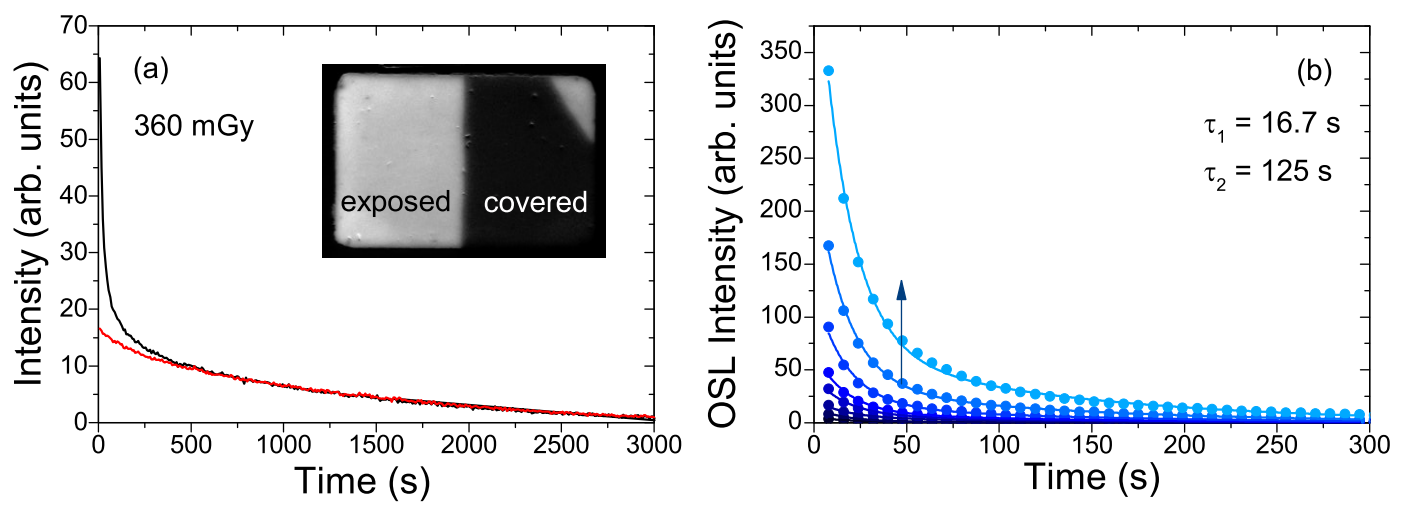

Figure 6.6: (a) PL + OSL decays measured for the irradiated segment of film after an Xray dose of $360 \mathrm{mGy}$ (black). Also shown is the PL decay as measured across the covered section of the film (red). Inset: X-ray image of the film after a dose of $360 \mathrm{mGy}$. (b) OSL decays for X-ray doses ranging from $20 \mathrm{mGy}$ to $2.88 \mathrm{~Gy}$ (symbols). The arrow indicates the trend for higher doses. Also shown are the two-exponential fits to the data (lines).

over a section of the sample during X-ray irradiation. The $\mathrm{Pb}$ attenuated $>99.99 \%$ of the $\mathrm{X}$-ray dose for each irradiation. The $\mathrm{Pb}$ piece was removed after irradiation, prior to $2 \mathrm{D}$ image collection. Thus, the uncovered section of the film effectively monitored the OSL + PL, while the covered section monitored only the PL component. The PL component was then subtracted from the OSL + PL curve, leaving only the OSL decay.

The film was characterised using the 2D dosimeter system, with $365 \mathrm{~nm}$ LED stimulation and $590 \mathrm{~nm}$ CCD camera detection. The aforementioned PL/OSL separation technique was employed in figure 6.6 (a), where the luminescence decays as a function of time during continuous stimulation are shown for both the covered and uncovered sections of the film after a $360 \mathrm{mGy}$ X-ray dose. The section of the film directly exposed to the X-rays fluoresced with approximately $4 \times$ the intensity of the $\mathrm{Pb}$ covered section immediately upon exposure to $365 \mathrm{~nm}$ light, demonstrating that the OSL component could be readily distinguished from the PL component. The luminescence from both sections decayed over the entire $3000 \mathrm{~s}$ period of stimulation, though the two decay components were equivalent after $\sim 550 \mathrm{~s}$. Therefore, the OSL component fully decayed over this initial $\sim 50 \mathrm{~s}$ stimulation period, such that longer stimulation times were unnecessary in collecting the entirety of the OSL signal.

The OSL decay curves, obtained by subtracting the luminescence decays of the covered section from the uncovered section of the film, are plotted in figure 6.6 (b) for X-ray doses ranging from $20 \mathrm{mGy}$ to $2.88 \mathrm{~Gy}$. The intensities at $t=0 \mathrm{~s}$ increased as a function of dose and the decays could be fitted to two-exponential decays (equation 6.1) for all 
doses. The decay constants as determined by fitting were $\tau_{1}=16.7 \mathrm{~s}$ and $\tau_{2}=125 \mathrm{~s}$. Similar decay constants were measured for the bulk sample when using the spectrofluorometer, though three components were observed and separated into fast, medium, and slow decay components (Section 4.3.4). The high intensity of the LED relative to that of the spectrofluorometer at $365 \mathrm{~nm}$ was expected to reduce all decay constants. Consequently, it was likely that the fast decay component could not be observed in the 2D OSL decays due to the decay constant being approximately equal to the image exposure time of $2 \mathrm{~s}$. The calculated decay constants therefore corresponded to the medium and slow components seen in the bulk compound. Using the known LED intensity and the OSL decay curves, the stimulation efficiency was calculated to be $0.1 \mathrm{~mJ} / \mathrm{mm}^{2}$. Consequently, over $65 \%$ of the OSL intensity could be collected in a single $30 \mathrm{~s}$ exposure.
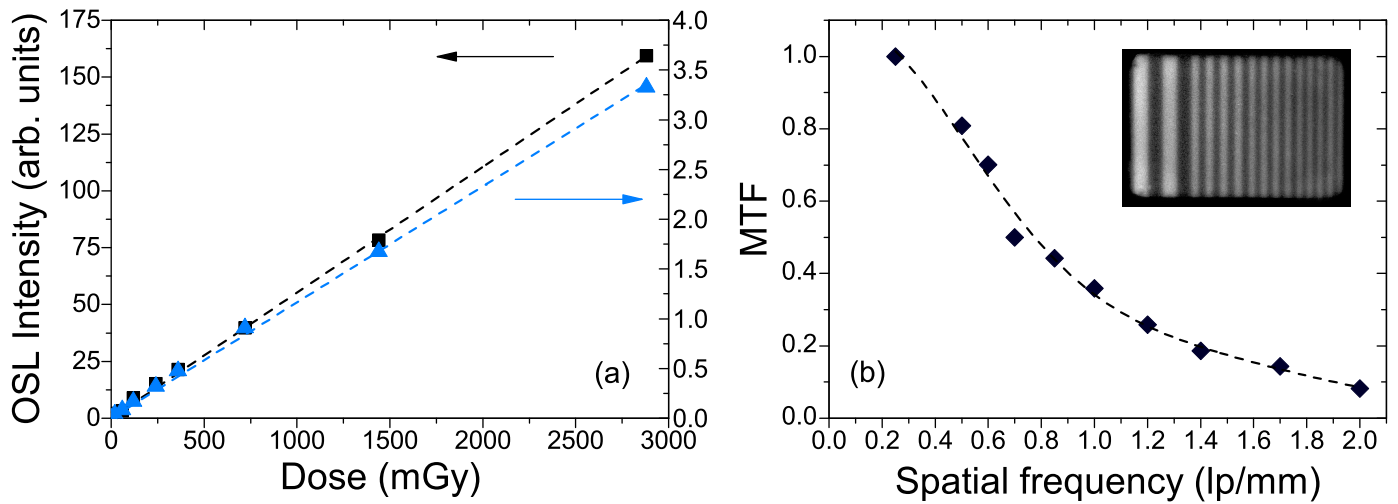

Figure 6.7: (a) Dose dependence of the integrated OSL intensity as integrated over the entire decay period and determined by fitting (black squares), and the OSL intensity obtained from the first $2 \mathrm{~s}$ exposure (blue triangles). The dashed lines are linear fits to the data. (b) MTF of the film. The dashed line is a guide to the eye. Inset: X-ray image of the film after an X-ray dose of $720 \mathrm{mGy}$ and using the $\mathrm{Pb}$ line phantom.

The dose dependence of the film OSL is shown in figure 6.7 (a) where two alternative integrations were used. In the first case, the integrated intensity of the entire OSL decay for each dose was calculated from the exponential fits. In the second case, only the intensity of the first image taken was used, corresponding to the first data point of each OSL decay curve i.e. a $2 \mathrm{~s}$ integration. In both cases the OSL intensity was linear from the low dose of $20 \mathrm{mGy}$ to the high dose of $2.88 \mathrm{~Gy}$. This demonstrated that the $2 \mathrm{D}$ system could be used for rapid 2D dose mapping, where a single readout required only $\sim 2 \mathrm{~s}$ of stimulation. However, the OSL as integrated over the entire decay increased the magnitude of the collected signal by over $40 \times$, implying a higher signal-to-noise ratio and greater measurement accuracy for longer exposure times. The two integration 
periods could be compared via noise equivalent dose calculations. Using the integrations over the entire decay, the noise equivalent dose was calculated to be $\sim 3 \mathrm{mGy}$. In contrast, using the first $2 \mathrm{~s}$ integration, the noise equivalent dose was calculated to be $\sim 7 \mathrm{mGy}$.

Finally, the spatial resolution of the $2 \mathrm{D}$ system using the $\mathrm{NaMgF}_{3}: \mathrm{Mn} / \mathrm{epoxy}$ composite films was characterised. The $\mathrm{Pb}$ phantom was used to mask the sample during X-ray irradiations where the dose was $720 \mathrm{mGy}$. The OSL images obtained after the first $2 \mathrm{~s}$ of each exposure were used to estimate the MTF of the system, and the MTF is plotted in figure 6.7 (d). At MTF = 0.2, the spatial frequency was approximately $1.4 \mathrm{lp} / \mathrm{mm}$, corresponding to sub-mm resolution.

\subsubsection{Summary and Conclusions}

This section demonstrated that the $2 \mathrm{D}$ system could be readily adapted to suit alternative OSL-active materials simply by switching out the LED stimulation source and the optical filters used in the system. It was also shown that the $\mathrm{NaMgF}_{3}$-based compounds could be mixed with an epoxy resin to produce reasonably homogeneous films useful for $2 \mathrm{D}$ imaging. In comparison to the bulk $\mathrm{NaMgF}_{3}: \mathrm{Eu}$ discussed in Section 6.2, the OSL emission of $\mathrm{NaMgF}_{3}: \mathrm{Mn}$ occurred at a longer wavelength than the stimulation, such that some PL was expected. It was shown that an additional PL contribution arose from the epoxy resin itself, and that this contribution exhibited a time-dependent intensity when continuously optically stimulated. This was corrected for by using a part of the sensing film as an internal reference, where a section of the film was covered by a $\mathrm{Pb}$ piece that effectively absorbed the entirety of the incident radiation. Via 2D imaging of the OSL + PL, the two components could be separated by subtracting the reference data, producing pure OSL decays that were characterised as a function of dose.

The key parameters of this configuration follow. The stimulation required to access $\sim 67 \%$ of the total stored OSL intensity was $0.1 \mathrm{~mJ} / \mathrm{mm}^{2}$. This is an exceptionally low stimulation efficiency that is over an order of magnitude lower than that of $\mathrm{NaMgF}_{3}: \mathrm{Eu}$ $\left(5.9 \mathrm{~mJ} / \mathrm{mm}^{2}\right)$ and of the commercial storage phosphor BaFBr:Eu $\left(16 \mathrm{~mJ} / \mathrm{mm}^{2}\right)$ [189]. A low stimulation efficiency suggests that rapid readout of the stored charges is possible, reducing the time required to take an OSL measurement and also the time required to optically reset the sample. The noise-equivalent doses were $\sim 3 \mathrm{mGy}$ for a full OSL readout and $\sim 7 \mathrm{mGy}$ for a $2 \mathrm{~s}$ readout. Both of these values satisfy the general $<10 \mathrm{mGy}$ requirement for radiotherapy quality assurance, though both could be improved via optimisation of the sensing material, particularly regarding the concentrations of defects and $\mathrm{Mn}^{2+}$, and the composition of the $\mathrm{NaMgF}_{3}: \mathrm{Mn} /$ epoxy composite. The limiting spa- 
tial resolution of the film was approximately $1.4 \mathrm{lp} / \mathrm{mm}$. Although sub-mm resolution was achieved, the spatial resolution was sharply reduced relative to the polycrystalline $\mathrm{NaMgF}_{3}:$ Eu compound, suggesting that the incorporation of epoxy may have increased the light scattering in the sample. The production of thin films with high $\mathrm{NaMgF}_{3}: \mathrm{Mn}$ loading fractions would greatly improve the imaging capabilities in terms of spatial resolution. Finally, the OSL response of the compound was linear over the dose range 20 $\mathrm{mGy}$ to $2.88 \mathrm{~Gy}$, indicating that a $\mathrm{NaMgF}_{3}: \mathrm{Mn}$ /epoxy composite could be used for radiotherapy dose verification.

\subsection{Development of the System}

In the previous sections it was demonstrated that 2D dose mapping was achievable using a relatively simple configuration, requiring only an OSL-active material (e.g. $\mathrm{NaMgF}_{3}: \mathrm{Mn}$ ), an LED source, and a CCD camera outfitted with specific optical filters. Using this simple configuration sub-mm resolutions were achieved and X-ray doses were detectable down to $<10 \mathrm{mGy}$.

It was desirable to develop the system further such that it was self-contained and portable. Per the previous discussions on the limitations of the original system, it was also encouraged that additional LEDs be incorporated into the system to increase the stimulation intensities and consequently reduce the readout times. The incorporation of several stimulating LEDs would also improve the homogeneity of the stimulating light.

The final system did not significantly differ regarding core functionality in comparison to the initial system. However, the robustness was vastly improved, as were the error-correcting capabilities such as those regarding reproducibility. Ultimately, the final system was suitable for demonstration in a medical context using the OSL-active compounds investigated throughout this work. The final version of the system is described below in its test-ready state. A majority of the custom components and electronics were designed and built by i2M labs.

An schematic diagram of the full system is shown in figure 6.8. The housing of the system was a custom built wooden box with dimensions $90 \mathrm{~cm} \times 34 \mathrm{~cm} \times 30 \mathrm{~cm}$, painted black to reduce internal reflections of the stimulation light. The lid of the box was fitted with a black foam inner lining to ensure that the system was light-tight i.e. no external lighting could enter the box and influence measurements. Some internal components were custom-designed and 3D printed using a LulzBot Mini 3D printer, where the material was $3 \mathrm{~mm}$ black ABS thermoplastic filament from Imagin Plastics. 


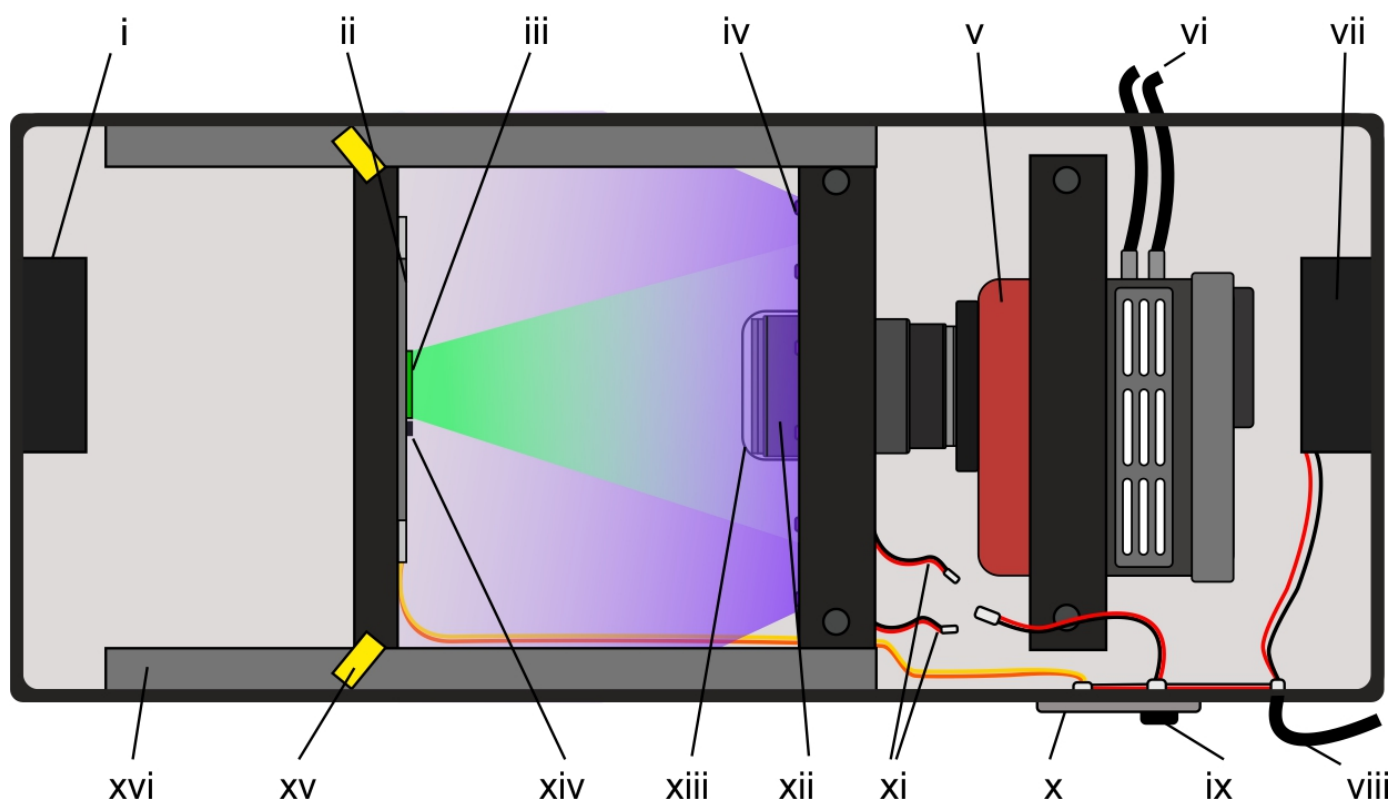

Figure 6.8: Illustration of the final 2D dosimeter system (not to scale). The view is of the internal components taken from above the system. The labeled components are discussed in the text.

Descriptions of each component as labeled in figure 6.8 follow:

i. Custom made (3D printed) vent. Featured a fold-back design in order to provide a light trap while maintaining air flow. Designed to allow for mounting of a $60 \mathrm{~mm}$ fan.

ii. Removable sample holder. The holder could be easily slotted into the system and subsequently removed. When the sample was fixed to the holder, the sample + holder could be removed for external X-ray irradiations and placed in the same position postirradiation.

iii. OSL-active sample used for dose monitoring.

iv. LED array featuring evenly spaced $6 \times 447.5 \mathrm{~nm}$ LUXEON Rebel LEDs and $6 \times$ OSRAM 368 nm LEDs.

v. SBIG STXL-11002 CCD camera.

vi. Outlet for connections from the camera to the power supply and PC.

vii. Custom made (3D printed) vent per (i). The vent was fitted with a $12 \mathrm{~V}$ DC $60 \mathrm{~mm}$ fan to ensure adequate airflow and heat ventilation throughout the system.

viii. Outlet allowing for connection of the fan, LED array, and photodiode to a power supply.

ix. Dial controlling the current supplied to the LED array.

x. Control circuits for the fan, LED array, and photodiode. 
xi. Connectors for the $447.5 \mathrm{~nm}$ LEDs and the $368 \mathrm{~nm}$ LEDs.

xii. Canon EF $35 \mathrm{~mm} \mathrm{f/1.4} \mathrm{lens} \mathrm{with} \mathrm{attachable} \mathrm{optical} \mathrm{filters.}$

xiii. Custom made (3D printed) lens cap used to mount additional optical filters.

xiv. BPW34 Si PIN photodiode.

$\mathrm{xv}$. Removable clamps used to position the modular components relative to the camera. xvi. Aluminium rails.
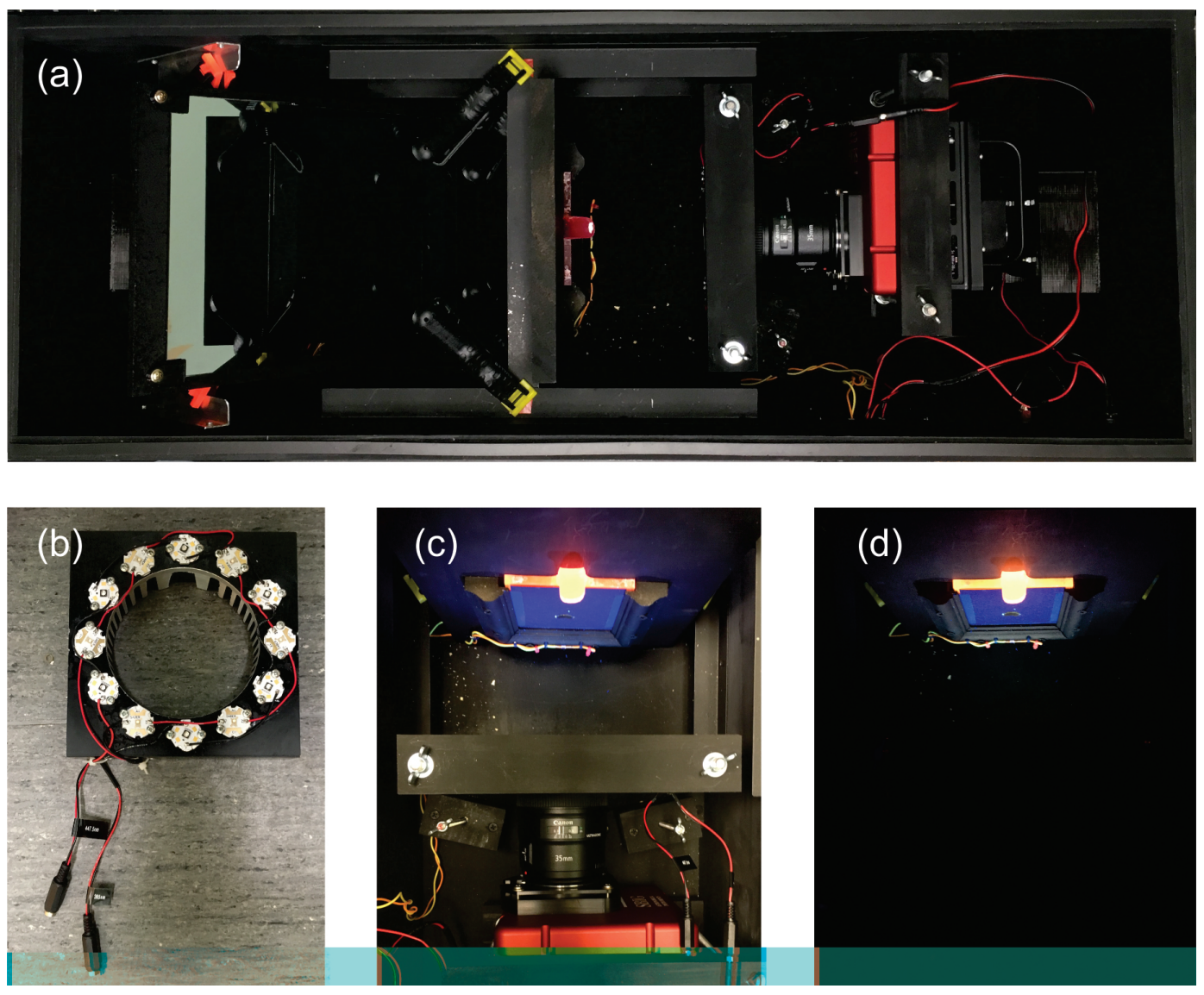

Figure 6.9: Photographs of the 2D dosimeter system. (a) Top-down view of the complete configuration per figure 6.8. A mirror was added to allow for eventual real-time inline measurements. (b) LED array composed of alternating $447.5 \mathrm{~nm}$ and $368 \mathrm{~nm}$ LEDs. (c) Image of the system and a fluoroperovskite sample under $368 \mathrm{~nm}$ stimulation with external lighting. (d) Image of the system and a fluoroperovskite sample under $368 \mathrm{~nm}$ stimulation without external lighting.

The final version was designed to be largely modular, such that individual components could be developed and improved over time without a complete overhaul of the system. The key additions to the system were the inclusion of the LED array and the incorporation of a photodiode for monitoring of the LED intensities over time. A pho- 
tograph of the completed system is shown in figure 6.9 (a), and a photograph of the LED array is shown in figure 6.9 (b). The use of multiple LEDs for stimulation increased the stimulation intensity significantly and therefore reduced the time required for OSL readout and total bleaching of the sensing materials. The photodiode was used to detect the intensity of the LED stimulation. A feedback circuit ensured the power supplied to the LEDs was proportional to the LED intensity as measured by the photodiode. The power supplied to the LEDs varied in time to satisfy the condition that the intensity was constant in time. This feature effectively negated the effects of temperature on the LED output. Finally, photographs of the active area of the system during $368 \mathrm{~nm}$ stimulation are shown in figures 6.9 (c) and (d) with and without external lighting, respectively.

\subsection{Discussion and Conclusions}

Dosimetry in 2D is necessary for radiotherapy dose verification, where high dose gradients may be encountered for specific treatment types [190,191]. Additionally, novel radiotherapy techniques such as microbeam radiation therapy [18] require highly sensitive $2 \mathrm{D}$ detectors with very high spatial resolutions. The common and conventionally used detectors are radiographic and radiochromic films, where exposure to ionising radiation permanently affects the optical properties of the film in a quantifiable way [190, 191]. As the effects are permanent, the films are single-use. Additionally, the films suffer from issues related to low signal stabilities over time and require precision handling.

The development of 2D dosimeters that operate using luminescence has largely focused on the two commercial materials $\mathrm{Al}_{2} \mathrm{O}_{3}: \mathrm{C}$ and $\mathrm{BeO}$. The most common readout technique involves a laser that raster scans the surface of a film post-irradiation, and the OSL intensity is read at each point. The laser scanning method is advantageous in that it allows for very high resolution measurements, as the size of the laser spot effectively dictates the resolution of the system [192]. However, the smaller the laser spot size the more measurements must be made in order to cover the entire sample. The time-perpoint is ultimately dictated by the fluorescence lifetime of the centre. If the laser moves to the next spot prior to the complete decay of the fluorescence from the previous point, pixel bleeding occurs that effectively reduces the resolution [59]. Ultimately, while laser scanning produces high-resolution 2D images, it is often associated with long readout times. Both $\mathrm{Al}_{2} \mathrm{O}_{3}: \mathrm{C}$ and $\mathrm{BeO}$ systems have suffered from this effect $[54,59]$.

The compounds studied in this chapter were $\mathrm{NaMgF}_{3}: \mathrm{Eu}$ and $\mathrm{NaMgF}_{3}: \mathrm{Mn}$. The PL lifetime of the main $\mathrm{Eu}^{2+}$ centre in $\mathrm{NaMgF}_{3}: \mathrm{Eu}$ is approximately $0.5 \mathrm{~ms}$, and the PL life- 
time of the non-distorted $\mathrm{Mn}^{2+}$ centre in $\mathrm{NaMgF}_{3}: \mathrm{Mn}$ is approximately $80 \mathrm{~ms}$. The use of either material in a high-resolution laser scanning system would necessitate long readout times. The use of a CCD camera system completely circumvented the influence of the PL lifetimes on the OSL readout times, where the camera exposures (i.e. integrations) were several orders of magnitude higher than the PL lifetimes. It was demonstrated that sensitive, accurate, and high-resolution images could be detected using the simple CCD camera configuration and a $\mathrm{NaMgF}_{3}: \mathrm{Mn} /$ epoxy composite in as little as $2 \mathrm{~s}$. This is a highly encouraging result and motivates additional studies into similar 2D systems using alternative OSL-active compounds.

As discussed in previous chapters, OSL is not the only luminescence mechanism by which radiation doses could be monitored using doped $\mathrm{NaMgF}_{3}$. Further development of the 2D system would also allow for simultaneous RPL measurements post-irradiation, and potentially real-time, in-line RPL and RL. For RPL measurements, a camera attachment featuring a filter wheel with different bandpass filters relevant to each RPL emission would be required. Real-time measurements are more difficult, as the system needs to be mounted in the radiation beam line. However, this could be achieved through the use of mirrors to direct the signal from an externally mounted film to the camera optics. Initial development of an in-line readout attachment is underway, where the sample is to be externally mounted and the luminescence directed to the CCD camera using mirrors.

For a material to be useful for radiotherapy dose verification it must satisfy three conditions. The first is that the material shows a well-behaved response to radiation doses ranging from several $\mathrm{mGy}$ to tens of $\mathrm{Gy}[9,190]$. Both materials tested in the 2D system, $\mathrm{NaMgF}_{3}: \mathrm{Eu}$ and $\mathrm{NaMgF}_{3}: \mathrm{Mn}$, exhibited integrated OSL responses that satisfied this requirement. The second condition is that spatial variations in the dose can be monitored to below $1 \mathrm{~mm}$. The spatial resolutions of the bulk $\mathrm{NaMgF}_{3}: \mathrm{Eu}$ and $\mathrm{NaMgF}_{3}: \mathrm{Mn} /$ epoxy film were $2.9 \mathrm{lp} / \mathrm{mm}$ and $1.4 \mathrm{lp} / \mathrm{mm}$, respectively. The final condition is that the uncertainty in the measured dose must be below 3\%, though ideally less than $1 \%$. The uncertainties in the data presented herein arose largely from uncertainties in the dose delivered from the X-ray sets, such that this parameter could not be reliably established.

All properties investigated in this chapter were based on the initial testing of nonoptimised materials and using a simple system. As the non-optimal setup met several of the key criteria required of a clinical dosimeter, it is expected that further development of the compounds and the readout system will result in a working product useful for application in radiotherapy quality assurance. Optimisation steps would include optimisation of the OSL-active compounds by controlling the defect concentrations, dopant concen- 
trations, and particle sizes. Furthermore, incorporation of the OSL-active materials into thin, uniform films would likely improve the spatial resolutions, where maximising the volume fraction of the OSL-active compound would be ideal. These steps would also improve the dose sensitivity of the system. Finally, the system requires characterisation in a medical context using the high-energy beams generated by linear accelerators. The results of the medical characterisation would likely motivate additional optimisation steps and determine the commercial viability of such a system for medical applications. 



\section{Chapter 7}

\section{Conclusions}

In the simplest sense, the intention of this work was to investigate the luminescence properties of some fluoroperovskite compounds. The luminescence properties were evaluated in terms of their potential applications with emphasis placed on applications in medical dosimetry. The main materials of interest were the doped $\mathrm{NaMgF}_{3}$ compounds, including $\mathrm{NaMgF}_{3}: \mathrm{Mn}$ and $\mathrm{NaMgF}_{3}: \mathrm{Ln}$ where $\mathrm{Ln}$ is any lanthanide. A wide range of luminescence phenomena were investigated throughout the work, most of which had some potential regarding radiation dose monitoring. These phenomena included OSL, RPL, RL, and TL.

The initial luminescence characterisations performed throughout this work were designed such that trends across compounds could be established and that systematic variations could be attributed to known physical phenomena. In doing so, the possible mechanisms responsible for the luminescence features before and after exposure to ionising radiation could be explored. Provided that the initial characterisations yielded promising results, compounds were studied in greater detail in order to gather more information on the various defects and traps, crystalline environments, and the energy levels of the luminescent ions. Finally, the most promising compounds were investigated in regards to their dosimetric properties, where the luminescence features were investigated as functions of X-ray dose.

Throughout this work, emphasis was placed on the development of novel techniques for passive dose monitoring that were not straightforward OSL or RPL measurements. The possibility of hybrid RPL/OSL dosimetry was investigated in some compounds and demonstrated to be a viable means of measuring radiation doses. The investigation of new techniques for active dose monitoring was also encouraged and real-time RPL, RL, and RIC methods were presented. An additional aspect of this work involved the devel- 
opment of a prototype dosimeter readout system that could monitor radiation doses in two-dimensions with good sensitivities and high spatial resolutions.

Presented in Chapter 4 was a comprehensive luminescence study of the four Mndoped compounds $\mathrm{NaMgF}_{3}: \mathrm{Mn}, \mathrm{KMgF}_{3}: \mathrm{Mn}, \mathrm{RbMgF}_{3}: \mathrm{Mn}$, and $\mathrm{CsCdF}_{3}: \mathrm{Mn}$. In all compounds $\mathrm{Mn}^{2+}$ luminescence was observed upon stimulation into the various crystal field split $3 d^{5}$ levels via a single radiative transition, namely ${ }^{4} \mathrm{~T}_{1 \mathrm{~g}}(\mathrm{G}) \rightarrow{ }^{6} \mathrm{~A}_{1 \mathrm{~g}}(\mathrm{~S})$. The energy levels of the $\mathrm{Mn}^{2+}$ ion were shown to correlate with the crystal fields of the host compounds, such that the crystal field splitting energies were described by $10 D q \propto R_{\mathrm{Mn}-\mathrm{F}}^{-4.48}$ where $R_{\mathrm{Mn}-\mathrm{F}}$ were the approximate $\mathrm{Mn}^{2+}-\mathrm{F}^{-}$bond lengths. Both oxygen-related and Ftype centre defects were observed in all samples and literature references were used to argue that the oxygen-related centres were likely $\mathrm{O}^{2-}-\mathrm{V}_{\mathrm{F}}$ dipoles. OSL was observed in all samples and the F-type centres were the traps responsible for the OSL in most cases. F-centre/Mn complexes were seen after irradiation in all $A M g F_{3}: M n$ compounds and the complexes could be used for RPL dosimetry. The energy levels of the complexes were experimentally determined and a systematic analysis of the structures for the different compounds yielded information that encourages renewed theoretical efforts in describing the physics of the complexes. Near tissue-equivalent $\mathrm{NaMgF}_{3}: \mathrm{Mn}$ was characterised regarding its dosimetric properties. It was demonstrated that $\mathrm{NaMgF}_{3}: \mathrm{Mn}$ could function as a hybrid OSL/RPL dosimeter, where cumulative doses could be non-destructively monitored up to at least $5 \mathrm{kGy}$ using RPL, and total doses up to approximately $180 \mathrm{~Gy}$ using OSL. Additionally, $\mathrm{CsCdF}_{3}: \mathrm{Mn}$ was shown to be a potential UV + X-ray dosimeter, where the charge kinetics for the two distinct stimulations were similar in that defects were produced that were OSL-active.

Chapter 5 presented a study into various $\mathrm{NaMgF}_{3}: \mathrm{Ln}$ compounds, where $\mathrm{Ln}=\mathrm{Sm}$, $\mathrm{Eu}$, Dy, or $\mathrm{Yb}$. The PL characterisations revealed RPL in the form of a radiation-induced valence conversion for Sm, Dy, and $\mathrm{Yb}$, where $\mathrm{X}$-ray irradiation resulted in the reduction of the ions from the trivalent to divalent states. The relative concentrations of each valence could be probed using PL spectroscopy and the concentrations were proportional to the radiation doses delivered. As the divalent $\mathrm{Dy}^{2+}$ ion is relatively rare in nature, a complete characterisation of the various $4 f^{9}$ and $4 f^{8} 5 d^{1}$ levels was presented and should serve as a valuable reference in future studies of the ion. The observation of $\mathrm{O}^{2-} \rightarrow \mathrm{Ln}^{3+}$ charge transfer bands for $\mathrm{Ln}=\mathrm{Sm}, \mathrm{Eu}$, and $\mathrm{Yb}$ motivated an attempt to establish the ground and excited state energies for all $\mathrm{Ln}^{3+}$ and $\mathrm{Ln}^{2+}$ ions relative to the host bands of $\mathrm{NaMgF}_{3}$ using the host-referred binding energy technique. This was ultimately successful and represents a novel means of calculating the binding energies for each Ln dopant 
that should be particularly useful in the study of fluoride compounds with large band gap energies. $\mathrm{NaMgF}_{3}: \mathrm{Sm}$ was selected as the most promising dosimeter compound and was characterised regarding its dosimetric properties. The compound was shown to have potential applications as an OSL/RPL hybrid dosimeter, though where the two phenomena were largely independent. Destructive OSL measurements could be made for individual doses, while the cumulative dose could be non-destructively monitored via the highlystable RPL. F-type centres were responsible for the OSL and emission occurred only via $\mathrm{Sm}^{3+}$ centres. The unusual dose-dependence of the RL was successively described using an adapted RL model that provides a basis for future modeling of similar OSL-active compounds featuring Ln dopants. It was experimentally demonstrated that the thermal quenching of the $\mathrm{Sm}^{2+}$ luminescence in the compound was related to the excitation of $\mathrm{Sm}^{2+}$ electrons to the conduction band. Thus, thermal quenching measurements can provide a new method with which one can determine the trap depths of $\mathrm{Ln}^{2+}$ ions. This method should be incredibly valuable in the assessment of RPL materials that operate using the valence conversion of $\mathrm{Ln}$ ions. Finally, it was demonstrated that $\mathrm{NaMgF}_{3}: \mathrm{Sm}_{\text {can }}$ also operate as an electronic dosimeter via the detection of radiation-induced currents. An experiment was constructed such that the RL and RIC were monitored simultaneously, demonstrating that the compound could be useful for hybrid optical/electrical dosimetry.

Chapter 6 summarised the demonstration and development of a 2D dosimeter readout system. The materials $\mathrm{NaMgF}_{3}: \mathrm{Eu}$ and $\mathrm{NaMgF}_{3}: \mathrm{Mn}$ were selected for 2D OSL characterisations. Both materials when integrated into the system showed good dose sensitivities and high spatial resolutions. The system used LEDs for OSL stimulation and a CCD camera for detection of the OSL emission. Further development of the system into a fully integrated, modular, and portable device was discussed, and it was ultimately concluded that the system presents several advantages over alternative 2D systems. Perhaps most strikingly, the system was capable of producing accurate 2D dose maps after only several seconds of readout.

In the introduction to this work, three main intentions were stated. The first was that various techniques should be established that could quantifiably monitor radiation doses. This was achieved in several novel ways, for example using the hybrid OSL + F/Mn complex RPL in $\mathrm{NaMgF}_{3}: \mathrm{Mn}$, the hybrid OSL $+\mathrm{Sm}^{3+} / \mathrm{Sm}^{2+} \mathrm{RPL}$ in $\mathrm{NaMgF}_{3}: \mathrm{Sm}$, or the hybrid RL + RIC in $\mathrm{NaMgF}_{3}: \mathrm{Sm}$. The second intention was to gain insight into the mechanisms driving the radiation sensing capabilities of the compounds. This was achieved where possible. A systematic analysis of the $A \mathrm{MgF}_{3}: \mathrm{Mn}$ compounds regarding 
the F/Mn complex energy levels was performed, a method of calculating HRBEs using $\mathrm{O}^{2-}$ impurities was demonstrated, and a method of calculating trap depths using the thermal quenching of divalent lanthanides was given. The third intention was to show that the compounds investigated herein are capable of 2D dose monitoring and are viable candidates for further development into commercial medical devices. This was achieved, as it was shown that the OSL of the compounds $\mathrm{NaMgF}_{3}: \mathrm{Eu}$ and $\mathrm{NaMgF}_{3}: \mathrm{Mn}$ could be used to map radiation in 2D with good accuracy in terms of both radiation dose and spatial variations. The work contained herein provides a solid foundation upon which further work optimising and developing both the compounds and the readout device may be built.

\section{Future Work}

The data and discussions throughout this work encourage further studies of the doped fluoroperovskites regarding both the luminescence features and the radiation sensing properties. Some possible directions of future research are summarised below.

The samples studied in this work were not optimised regarding dopant or defect concentrations. Both concentrations could be controlled via specific synthesis techniques, where the intentional addition of alternative dopant ions such as $\mathrm{K}^{+}, \mathrm{Li}^{+}$, or $\mathrm{O}^{2-}$ could affect the F-type centre concentrations and consequently the OSL sensitivities in the $\mathrm{NaMgF}_{3}$-based compounds. In the case of Ln dopants, the use of reducing or oxidising agents during synthesis, or the inclusion of dopant precursors in their oxide forms $\left(\mathrm{Ln}_{2} \mathrm{O}_{3}\right)$, could potentially alter the ratio of $\mathrm{Ln}^{2+} / \mathrm{Ln}^{3+}$ dopants in the samples, affecting the RPL sensitivities. The introduction of different defects and their influence on the local symmetries of dopant ions may also improve the stabilities of the structures responsible for the RPL, and increase the rate at which charge trapping occurs. It would therefore be of great interest to perform a study where the stoichiometry of the precursor fluorides and the concentrations of the dopants were systematically varied for the most promising compounds.

Some structures observed in the different compounds warrant further experimental and theoretical analyses. The oxygen-related defects could be investigated in greater detail experimentally by deliberately introducing different oxygen concentrations into the lattice. Electron spin resonance and extended X-ray absorption fine structure techniques could be employed to better understand the structures of the centres. Likewise, the nature of the site distortion observed for one $\mathrm{Mn}^{2+}$ site in $\mathrm{NaMgF}_{3}: \mathrm{Mn}$ could be further investigated using similar techniques. The experimental analysis of the F/Mn 
complexes inspires renewed theoretical efforts to describe the electronic structures of the complexes, and also encourages a search for similar transition metal + F-centre complexes in different materials.

Some analysis techniques developed throughout this work should be useful in the analysis of other compounds. The model employed to describe the $\mathrm{Sm}^{2+} / \mathrm{Sm}^{3+} \mathrm{RL}$ should be tested using alternative $\mathrm{Ln}^{2+} / \mathrm{Ln}^{3+}$ dopants in $\mathrm{NaMgF}_{3}: \mathrm{Ln}$ and other host compounds. The thermal quenching technique should be used for $\mathrm{Ln}^{2+}$ ions other than $\mathrm{Sm}^{2+}$. Additionally, it would be of interest to establish a HRBE diagram for $\mathrm{NaMgF}_{3}: \mathrm{Ln}$ using the $\mathrm{F}^{-}$ $\rightarrow \mathrm{Ln}^{3+}$ charge transfer bands and compare the results to those obtained from the $\mathrm{O}^{2-} \rightarrow$ $\mathrm{Ln}^{3+}$ charge transfer bands. Such a study would require access to a versatile VUV source coupled to a spectrometer.

Finally, to be deemed truly suitable for applications in medical dosimetry, selected compounds require characterisations using the MV X-rays used for radiotherapy. While the qualitative effects of the irradiations should not significantly differ from those discussed herein, it is possible that higher X-ray energies could produce additional defects in the compounds and/or affect the dose ranges over which the luminescence features are linear. Similarly, the compounds and films used for 2D dosimetry should be characterised using MV X-rays and using the 2D system for readout. In performing these characterisations, techniques specific to the materials of interest will be developed, such that the compounds may eventually be employed in a clinical dosimetry context. 



\section{References}

[1] H. Fraga, Firefly luminescence: A historical perspective and recent developments, Photochem. Photobiol. Sci 7 (2008) 146-158.

[2] R. Boyle, Experiments and considerations touching colours, Transactions of the Royal Society (1664).

[3] G. Blasse, B. C. Grabmaier, A General Introduction to Luminescent Materials. In: Luminescent Materials, Springer, Berlin, Heidelberg, 1994.

[4] T. Li, Z. Wang, P. Li, S. Xu, Q. Bai, Z. Yang, A novel red emitting phosphor $\mathrm{LiBaB}_{9} \mathrm{O}_{15}: \mathrm{Sm}^{2+} / \mathrm{Sm}^{3+}, \mathrm{Li}^{+}$with broad excitation band for white LEDs, Luminescence 33 (2017) 438-442.

[5] A. Ikesue, Polycrystalline Nd:YAG ceramics lasers, Opt. Mater. 19 (2002) 183-187.

[6] S. Schweizer, U. Rogulis, S. Assman, K. J. M. Spaeth, RbBr and CsBr doped with $\mathrm{Eu}^{2+}$ as new competitive X-ray storage phosphors, Radiat. Meas. 33 (2001) 483486.

[7] Y. Zhuang, J. Ueda, S. Tanabe, Multi-color persistent luminescence in transparent glass ceramics containing spinel nano-crystals with $\mathrm{Mn}^{2+}$ ions, Appl. Phys. Lett. 105 (2014) 191904.

[8] E. G. Yukihara, S. W. S. McKeever, Optically Stimulated Luminescence Fundamentals and Applications, John Wiley and Sons, UK, 2011.

[9] S. W. S. McKeever, M. Moscovitch, P. D. Townsend, Thermoluminescence dosimetry materials: properties and uses, Nuclear Technology Publishing, Ashford, U.K., 1995.

[10] W. C. Röntgen, On a new kind of rays, Nature 53 (1896) 274-276. 
[11] H. Becquerel, Sur les radiations èmises par phosphorescence, Compt. Ren. 122 (1896) 420.

[12] P. Curie, M. Curie, G. Bèmont, Sur une nouvelle substance fortement radio-active, contenue dans la pechblende, Compt. Ren. 127 (1898) 1215.

[13] M. Akatsuka, Y. Usui, D. Nakauchi, T. Kato, N. Kawano, G. Okada, N. Kawaguchi, T. Yanagida, Scintillation properties of $\mathrm{YAlO}_{3}$ doped with $\mathrm{Lu}$ and $\mathrm{Nd}$ perovskite single crystals, Opt. Mater. 79 (2018) 428-434.

[14] F. A. Mettler, P. W. Wiest, J. A. Locken, C. A. Kelsey, CT scanning: patterns of use and dose, J. Radiol. Prot. 20 (2000) 353-359.

[15] G. V. M. Williams, S. G. Raymond, Fiber-optic-coupled $\mathrm{RbMgF}_{3}: \mathrm{Eu}^{2+}$ for remote radiation dosimetry, Radiat. Meas. 46 (2011) 1099-1102.

[16] B. G. Haffty, L. D. Wilson, Handbook of Radiation Oncology, Jones and Bartlett Publishers, 2009.

[17] S. Derreumaux, C. Etard, C. Huet, F. Trompier, I. Clairand, J. F. Bottollier-Depois, B. Aubert, P. Gourmelon, Lessons from recent accidents in radiation therapy in France, Radiat. Prot. Dosim. 131 (2008) 130-135.

[18] G. Okada, B. Morell, C. Koughia, A. Edgar, C. Varoy, G. Belev, T. Wysokinski, D. Chapman, S. Kasap, Spatially resolved measurement of high doses in microbeam radiation therapy using samarium doped fluorophosphate glasses, Appl. Phys. Lett. 99 (2011) 121105.

[19] F. Daniels, C. A. Boyd, D. F. Saunders, Thermoluminescence as a research tool, Science 117 (1953) 343-349.

[20] J. Zimmerman, The radiation-induced increase of thermoluminescence sensitivity of the dosimetry phosphor LiF(TLD-100), J. Phys. C: Solid St. Phys. 4 (1971) 32773291.

[21] R. Gaza, S. McKeever, M. Akselrod, Near-real-time radiotherapy dosimetry using optically stimulated luminescence of $\mathrm{Al}_{2} \mathrm{O}_{3}: \mathrm{C}$ : Mathematical models and preliminary results, Med. Phys. 32 (2005) 1094-1102. 
[22] A. V. Dmitryuk, S. E. Paramzina, A. S. Perminov, N. D. Solov'eva, N. T. Timofeev, The influence of glass composition on the properties of silver-doped radiophotoluminescent phosphate glasees, J. Non-cryst. Solids 202 (1996) 173-177.

[23] J. C. Gacon, A. Gros, H. Bill, J. P. Wicky, New Measurements of the Emission Spectra of $\mathrm{Sm}^{2+}$ in $\mathrm{KMgF}_{3}$ and $\mathrm{NaMgF}_{3}$ Crystals, J. Phys. Chem. Solids 42 (1981) 587-593.

[24] C. Furetta, G. Ramogida, A. Scacco, M. Martini, S. Paravisi, Spectroscopy of complex defects in crystals of $\mathrm{KMgF}_{3}: \mathrm{Tl}^{+}$, J. Phys. Chem. Solids 55 (1994) 1337-1345.

[25] D. K. Sardar, W. A. Sibley, R. Alcala, Optical absorption and emission from irradiated $\mathrm{RbMgF}_{3}: \mathrm{Eu}^{2+}$ and $\mathrm{KMgF}_{3}: \mathrm{Eu}^{2+}$, J. Lumin. 27 (1982) 401-411.

[26] W. A. Sibley, S. I. Yun, W. E. Vehse, Colour centre luminescence in $\mathrm{KMgF}_{3}: \mathrm{Mn}$ crystals, J. Phys. C: Solid State Phys. 6 (1973) 1105-1115.

[27] G. V. M. Williams, S. Janssens, C. Gaedtke, S. G. Raymond, D. Clarke, Observation of photoluminescence and radioluminescence in $\mathrm{Eu}$ and $\mathrm{Mn}$ doped $\mathrm{NaMgF}_{3}$ nanoparticles, J. Lumin. 143 (2013) 219-225.

[28] C. Dotzler, G. V. M. Williams, U. Rieser, A. Edgar, Optically stimulated luminescence in $\mathrm{NaMgF}_{3}: \mathrm{Eu}^{2+}$, Appl. Phys. Lett. 91 (2007) 121910.

[29] C. J. Dotzler, G. V. M. Williams, A. Edgar, Thermoluminescence, photoluminescence and optically stimulated luminescence properties of X-ray irradiated $\mathrm{RbMgF}_{3}: \mathrm{Mn}^{2+}$, Phys. Stat. Sol. C 4 (2007) 992-995.

[30] J. Donaldson, G. V. M. Williams, Photoluminescence, radioluminescence, and thermoluminescence in $\mathrm{NaMgF}_{3}$ activated with $\mathrm{Ni}^{2+}$ and $\mathrm{Er}^{3+}$, J. Lumin. 173 (2016) 279-285.

[31] E. B. Podgorsak, Radiation Physics for Medical Physicists, Springer, London, 2010.

[32] F. H. Attix, Introduction to radiological physics and radiation dosimetry, John Wiley and Sons, UK, 1986.

[33] J. H. Hubbell, S. M. Seltzer, Tables of X-ray mass attenuation coefficients and mass energy-absorption coefficients from $1 \mathrm{keV}$ to $20 \mathrm{MeV}$ for elements $\mathrm{Z}=1$ to 92 and 48 additional substances of dosimetric interest (version 1.4), National Institute of Standards and Technology, Gaithersburg, MD, 2004. 
[34] F. A. Mettler, Medical effects and risks of exposure to ionising radiation, J. Radiol. Prot. 32 (2012) N9-N19.

[35] N. E. Bolus, Basic Review of Radiation Biology and Terminology, J. Nucl. Med. Technol. 29 (2001) 67-73.

[36] M. I. Nunez, T. J. McMillan, M. T. Valenzuela, J. M. R. de Almodovar, V. Pedraza, Relationship between DNA damage, rejoining and cell killing by radiation in mammalian cells, Radiotherapy and Oncology 39 (1996) 155-165.

[37] N. Schreuder, D. Koopman, P. L. Jager, J. G. W. Kosterink, E. van Puijenbroek, Adverse Events of Diagnostic Radiopharmaceuticals: A Systematic Review, Semin. Nucl. Med. 49 (2019) 382-410.

[38] W. P. M. Mayles, R. Lake, A. McKenzie, E. M. Macaulay, H. M. Morgan, T. J. Jordan, S. K. Powley, Physics Aspects of Quality Control in Radiotherapy, The Institute of Physics and Engineering in Medicine, York, 1999.

[39] A. Jahn, M. Sommer, W. Ullrich, M. Wickert, J. Henniger, The BeOmax system - Dosimetry using OSL of BeO for several applications, Radiat. Meas. 56 (2013) 324-327.

[40] J. M. Havercroft, S. C. Klevenhagen, Ion recombination corrections for planeparallel and thimble chambers in electron and photon radiation, Phys. Med. Biol. 38 (1993) 25-38.

[41] M. Lavallee, L. Gingras, L. Beaulieu, Energy and integrated dose dependence of MOSFET dosimeter sensitivity for irradiation energies between $30 \mathrm{kV}$ and ${ }^{60} \mathrm{Co}$, Med. Phys. 33 (2006) 3683-3689.

[42] J. J. Schuyt, G. V. M. Williams, The effect of ionizing radiation on the optical properties of $\mathrm{NaMgF}_{3}(\mathrm{Mn})$ : Observation of an F-center Mn complex, J. Appl. Phys. 122 (2017) 063107.

[43] J. J. Schuyt, G. V. M. Williams, Radiation-induced changes in the optical properties of $\mathrm{NaMgF}_{3}(\mathrm{Sm})$ : Observation of resettable Sm radio-photoluminescence, Mater. Res. Bull. 106 (2018) 455-458.

[44] J. J. Schuyt, G. V. M. Williams, Development of a 2D dosimeter using the optically stimulated luminescence of $\mathrm{NaMgF}_{3}: \mathrm{Eu}$ with $\mathrm{CCD}$ camera readout, Radiat. Meas. 121 (2019) 99-102. 
[45] L. C. Oliviera, E. G. Yukihara, O. Baffa, MgO:Li,Ce,Sm as a high-sensitivity material for Optically Stimulated Luminescence dosimetry, Nature Scientific Reports (2016).

[46] P. Molina, M. Prokic, J. Marcazzo, M. Santiago, Characterization of a fiberoptic radiotherapy dosimetry probe based on $\mathrm{Mg}_{2} \mathrm{SiO}_{4}: \mathrm{Tb}$, Radiation Measurements 45 (2010) 78-82.

[47] P. Molina, M. Santiago, J. Marcasso, E. Caselli, M. Prokic, N. Khaidukov, C. Furetta, Characterisation of new materials for fiberoptic dosimetry, J. Phys. Conference Series 313 (2011) 012007.

[48] D. J. Daniel, A. Raja, U. Madhusoodanan, O. Annalakshmi, P. Ramasamy, OSL studies of alkali fluoroperovskite single crystals for radiation dosimetry, Opt. Mater. 58 (2016) 497-503.

[49] F. X. Masse, Film Badge Dosimetry in Atmospheric Nuclear Tests, National Academy Press, Washington, D.C., 1989.

[50] F. Marsolat, D. Tromson, N. Tranchant, M. Pomorski, M. L. Roy, M. Donois, F. Moignau, A. Ostrowsky, L. D. Carlan, C. Bassinet, C. Huet, S. Derreumaux, M. Chea, K. Cristina, G. Boisserie, P. Bergonzo, A new single crystal diamond dosimeter for small beam: comparison with different commercial active detectors, Phys. Med. Biol. 58 (2013) 7647-7660.

[51] B. Gorka, B. Nilsson, R. Svensson, A. Brahme, P. Ascarelli, D. M. Trucchi, G. Conte, R. Kalish, Design and characterization of a tissue-equivalent CVD-diamond detector for clinical dosimetry in high-energy photon beams, Physica Medica 24 (2008) 159.

[52] A. Fidanzio, L. Azario, C. Venanzi, F. Pinzari, A. Piermattei, Production and testing of a synthetic diamond film radiation dosimeter for radiotherapy, Nucl. Instrum. Meth. Phys. Res. A 479 (2002) 661-667.

[53] M. Ptaszkiewicz, E. Braurer-Kirsch, M. Klosowski, L. Czopyk, P. Olko, TLD dosimetry for microbeam radiation therapy at the European Synchrotron Radiation Facility, Radiat. Meas. 43 (2008) 990-993.

[54] A. Jahn, M. Sommer, M. Liebmann, J. Henniger, Progress in 2D-OSL-dosimetry with beryllium oxide, Radiat. Meas. (46) (2011) 1908-1911. 
[55] B. Henderson, G. Imbusch, Optical Spectroscopy of Inorganic Solids, Oxford University Press, New York, 2006.

[56] S. Gasiorowicz, Quantum Physics, John Wiley and Sons, New York, 1974.

[57] W. A. Sibley, N. Koumvakalis, Low-temperature radiation damage in Mn-doped $\mathrm{RbMgF}_{3}$, J. Phys. C: Solid State Phys. 10 (1977) 4909-4919.

[58] Y. Miyamoto, Y. Takei, H. Nanto, T. Kurobori, A. Konnai, T. Yanagida, A. Yoshikawa, Y. Shimotsuma, M. Sakakura, K. Miura, J. Hirao, Y. Nagashima, T. Yamamoto, Radiophotoluminescence from silver-doped phosphate glass, Radiat. Meas. 46 (2011) 1480-1483.

[59] M. F. Ahmed, S. A. Eller, E. Schnell, S. Ahmad, M. S. Akselrod, O. D. Hanson, E. G. Yukihara, Development of a 2D dosimetry system based on the optically stimulated luminescence of $\mathrm{Al}_{2} \mathrm{O}_{3}$, Radiat. Meas. 71 (2014) 187-192.

[60] V. Pagonis, J. Lawless, R. Chen, C. Andersen, Radioluminescence in $\mathrm{Al}_{2} \mathrm{O}_{3}: \mathrm{C}$ - analytical and numerical simulation results, J. Phys. D: Appl. Phys. 42 (2009) 175107.

[61] J. S. Souris, S.-H. Cheng, C. Pelizzari, N.-T. Chen, P. L. Riviere, C.-T. Chen, L.-W. Lo, Radioluminescence characterization of in situ x-ray nanodosimeters: potential real-time monitors and modulators of external beam radiation therapy, Appl. Phys. Lett. 105 (2014) 203110.

[62] J. J. Schuyt, G. V. M. Williams, Photoluminescence, radioluminescence and optically stimulated luminescence in nanoparticle and bulk $\mathrm{KMgF}_{3}(\mathrm{Eu})$, J. Lumin. 204 (2018) 472-479.

[63] R. Gaza, S. W. S. McKeever, M. S. Akselrod, A. Akselrod, T. Underwood, C. Yoder, C. E. Andersen, M. C. Aznar, C. Marckmann, L. Botter-Jensen, A fiber-dosimetry method based on OSL from $\mathrm{Al}_{2} \mathrm{O}_{3}: \mathrm{C}$ for radiotherapy applications, Radiat. Meas. 38 (2004) 809-812.

[64] E. A. Burgemeister, Dosimetry with a diamond operating as a resistor, Phys. Med. Biol. 26 (2) (1981) 269.

[65] Y. Tanabe, S. Sugano, On the absorption spectra of complex ions II, J. Phys. Soc. Jap. 9 (1954) 766-779. 
[66] N. M. Winch, A. Edgar, X-ray imaging using a consumer-grade digital camera, Nucl. Instrum. Meth. Phys. Res. A 654 (2011) 308-313.

[67] P. Dorenbos, $\mathrm{Ce}^{3+} 5 \mathrm{~d}$-centroid shift and vacuum referred 4 f-electron binding energies of all lanthanide impurities in 150 different compounds, J. Lumin. 135 (2013) 93-104.

[68] P. Dorenbos, The electronic level structure of lanthanide impurities in $\mathrm{REPO}_{4}$, $\mathrm{REBO}_{3}, \mathrm{REAlO}_{3}$, and $\mathrm{RE}_{2} \mathrm{O}_{3}(\mathrm{RE}=\mathrm{La}, \mathrm{Gd}, \mathrm{Y}, \mathrm{Lu}, \mathrm{Sc}$ ) compounds, J. Phys.: Condens. Matter 25 (2013) 225501.

[69] P. Dorenbos, The $5 \mathrm{~d}$ level positions of the trivalent lanthanides in inorganic compounds, J. Lumin. 91 (2000) 155-176.

[70] P. Dorenbos, Locating lanthanide impurity levels in the forbidden band of host crystals, J. Lumin. 108 (2004) 301-305.

[71] G. H. Dieke, Spectra and energy levels of rare earth ions in crystals, Interscience Publishers, New York, 1968.

[72] M. Suta, C. Wickleder, Synthesis, spectroscopic properties and applications of divalent lanthanides apart from $\mathrm{Eu}^{2+}$, J. Lumin. 210 (2019) 210-238.

[73] G. Horsch, H. J. Paus, A new color center laser on the basis of lead doped $\mathrm{KMgF}_{3}$, Opt. Comm. $60(1,2)(1986) 69$.

[74] E. Song, Z. Chen, M. Wu, S. Ding, S. Ye, S. Zhao, Q. Zhang, Room-temperature wavelength-tunable single-band upconversion luminescence from $\mathrm{Yb}^{3+} / \mathrm{Mn}^{2+}$ codoped fluoride perovskites $\mathrm{ABF}_{3}$, Adv. Opt. Mater. 4 (2016) 798-806.

[75] W. A. Sibley, S. I. Yun, L. N. Feuerhelm, Radiation defect and 3d impurity absorption in $\mathrm{MgF}_{2}$ and $\mathrm{KMgF}_{3}$ crystals, J. Physique 34 (1973) C9-503-506.

[76] W. E. Vehse, W. A. Sibley, Radiation damage in $\mathrm{KMgF}_{3}: \mathrm{KMnF}_{3}$, Phys. Rev. B 6 (1972) 2443-2453.

[77] C. Dotzler, G. V. M. Williams, A. Edgar, $\mathrm{RbCdF}_{3}: \mathrm{Mn}^{2+}$ : A potential ultraviolet dosimeter material, Appl. Phys. Lett. 91 (2007) 181909. 
[78] J. Donaldson, G. V. M. Williams, A luminescence study of $\mathrm{NaMgF}_{3}: \mathrm{Dy}^{3+}$ and $\mathrm{NaMgF}_{3}: \mathrm{Nd}^{3+}$ for applications in radiation dosimetry, Int. J. Nanotechnol. 14 (2017) 558-565.

[79] H. Kronmuller, S. Parkin, Handbook of magnetism and advanced magnetic materials: Spintronics and magnetoelectronics, John Wiley and Sons, New York, 2007.

[80] T. Nishimatsu, N. Terakubo, H. Mizuseki, Y. Kawazoe, D. A. Pawlak, K. Shimamura, T. Fukuda, Band structures of perovskite-like fluorides for vacuum-ultraviolettransparent lens materials, Jpn. J. Appl. Phys. 41 (2002) L365-L367.

[81] R. D. Shannon, Revised Effective Ionic Radii and Systematic Studies of Interatomie Distances in Halides and Chaleogenides, Acta Cryst. 32 (1976) 751.

[82] R. H. Mitchell, M. Alexander, L. M. D. Cranswick, I. P. Swainson, A powder neutron diffraction study of the crystal structure of the fluoroperovskite $\mathrm{NaMgF}_{3}$ (neighbourite) from 300 to 3.6 K, Phys. Chem. Minerals 34 (2007) 705.

[83] R. B. Hughes-Currie, K. V. Ivanovskikh, M. F. Reid, J. R. Wells, R. J. Reeves, A. Meijerink, Vacuum ultraviolet synchrotron measurements of excitons in $\mathrm{NaMgF}_{3}: \mathrm{Yb}^{2+}$, J. Lumin. 169 (2016) 419-421.

[84] N. J. M. L. Masson, A. P. Vink, P. Dorenbos, A. J. J. Bos, C. W. E. V. Eijk, J. P. Chaminade, $\mathrm{Ce}^{3+}$ and $\mathrm{Pr}^{3+} 5$ d-energy levels in the (pseudo) perovskites $\mathrm{KMgF}_{3}$ and $\mathrm{NaMgF}_{3}$, J. Lumin. 101 (2003) 175-183.

[85] B. Trieman, N. Kristianpoller, Defects induced in $\mathrm{RbMgF}_{3}$ crystals by X- and UVirradiation, Phys. Stat. Sol. B 105 (1981) 739-746.

[86] M. Springis, A. Sharakovsky, I. Tale, U. Rogulis, X-irradiation induced photo- and thermostimulated luminescence of $\mathrm{CsCdF}_{3}: \mathrm{Mn}$ crystals, Phys. Stat. Sol. C 2 (2005) 511-514.

[87] E. Ronnebro, D. Noreus, K. Kadir, A. Reiser, B. Bogdanovic, Investigation of the perovskite related structures of $\mathrm{NaMgH}_{3}, \mathrm{NaMgF}_{3}$ and $\mathrm{Na}_{3} \mathrm{AlH}_{6}$, J. Alloys Compd. 299 (2000) 101-106.

[88] M. L. Falin, V. A. Latypov, A. M. Leushin, G. M. Safiullin, EPR and optical spectroscopy of $\mathrm{Yb}^{3+}$ ions in hexagonal perovskite $\mathrm{RbMgF}_{3}$ crystals, J. Alloy Compd. 735 (2018) 23-28. 
[89] G. Vaitheeswaran, V. Kanchana, R. S. Kumar, A. L. Cornelius, M. F. Nicol, A. Svane, N. E. Christensen, O. Eriksson, High-pressure structural study of fluoro-perovskite $\mathrm{CsCdF}_{3}$ up to $60 \mathrm{GPa}$ : A combined experimental and theoretical study, Phys. Rev. B 81 (2010) 075105.

[90] J. Donaldson, Radioluminescence of $\mathrm{NaMgF}_{3}$ : characterisation for radiotherapy, Thesis (2017).

[91] J. Punnoose, J. Xu, A. Sisniega, W. Zbijewski, J. H. Siewerdsen, Technical Note: SPEKTR 3.0 - A computational tool for x-ray spectrum modeling and analysis, Med. Phys. 43 (2016) 4711-4717.

[92] C. Dotzler, Optically stimulated luminescence in fluoroperovskites for dosimetric applications, Thesis (2008).

[93] S. Corporation, Shimadzu UV-Visible Spectrophotometer UV-2600/2700 Instruction Manual (2011).

[94] H. Scientific, Fluorolog-3 Operation Manual rev. G (2014).

[95] J. W. Quilty, J. Robinson, G. A. Appleby, A. Edgar, Thermoluminescence apparatus using PT100 resistors as the heating and sensing elements, Rev. Sci. Instrum. 78 (2007) 083905.

[96] E. van der Kolk, P. Dorenbos, C. W. E. van Eijk, A. P. Vink, M. Weil, J. P. Chaminade, Luminescence excitation study of the higher energy states of $\mathrm{Pr}^{3+}$ and $\mathrm{Mn}^{2+}$ in $\mathrm{SrAlF}_{5}, \mathrm{CaAlF}_{5}$, and $\mathrm{NaMgF}_{3}$, J. Appl. Phys. 95 (2004) 7867-7872.

[97] A. K. Kole, C. S. Tiwary, P. Kumbhakar, Room temperature synthesis of $\mathrm{Mn}_{2+}$ doped $\mathrm{ZnS}$ d-dots and observation of tunable dual emission: Effects of doping concentration, temperature, and ultraviolet light illumination, J. Appl. Phys. 113 (2013) 114308.

[98] J. Wang, Y. Li, Q. Shen, T. Izuishi, Z. Pan, K. Zhao, X. Zhong, Mn doped quantum dot sensitized solar celss with power conversion efficiency exceeding 9

[99] Y. Katayama, J. Ueda, S. Tanabe, Effect of $\mathrm{Bi}_{2} \mathrm{O}_{3}$ doping on persistent lumienscence of $\mathrm{MgGeO}_{3}: \mathrm{Mn}^{2+}$ phosphor, Opt. Mater. Express 4 (2014) 613-623. 
[100] G. V. M. Williams, C. Dotzler, A. Edgar, S. G. Raymond, Ultraviolet induced absorption and Bragg grating inscription in $\mathrm{RbCdF}_{3}: \mathrm{Mn}^{2+}$, J. Appl. Phys. 102 (2007) 113106.

[101] C. Dotzler, G. V. M. Williams, A. Edgar, S. Schweizer, B. Henke, J. M. Spaeth, A. Bittar, J. Hamlin, C. Dunford, The effect of x-ray, $\gamma$-ray, and UV radiations on the optical properties of $\mathrm{RbCdF}_{3}: \mathrm{Mn}^{2+}$, J. Appl. Phys. 100 (2006) 033102.

[102] H. Luo, J. Cao, X. Li, X. Wang, M. Peng, Topological tailoring of structure and defects to enhance red to near-infrared afterglow from $\mathrm{Mn}^{2+}$-doped germanate photonic glasses, J. Mater. Chem. C 6 (2018) 11525.

[103] M. C. M. de Lucas, F. Rodriguez, M. Moreno, Zero-phonon transitions and the Stokes shift of $\mathrm{Mn}^{2+}$-doped perovskites: Dependence on the metal-ligand distance, Phys. Rev. B 50 (1994) 2760-2765.

[104] H. Su, Z. Jia, C. Shi, Study of Color Centers and Trace Oxygen in $\mathrm{KMgF}_{3}: \mathrm{Eu}$ Single Crystal, Chem. Mater. 14 (2002) 310.

[105] T. P. P. Hall, A. Leggeat, Defect centres in $\mathrm{KMgF}_{3}$ produced by X-irradiation at room temperature, Solid State Comm. 7 (1969) 1657-1659.

[106] C. R. Riley, S. I. Yun, W. A. Sibley, Luminescence from color centers in $\mathrm{KMgF}_{3}$, Phys. Rev. B 5 (1972) 3285-3292.

[107] G. V. M. Williams, C. Dotzler, J. Robinson, Radiation-induced optically stimulated luminescence in $\mathrm{Mn}^{2+}$ and $\mathrm{Eu}^{2+}$ doped $\mathrm{RbMgF}_{3}$, Phys. Stat. Sol. C 6 (2009) S198S201.

[108] A. I. Popov, E. A. Kotomin, J. Maier, Basic properties of the F-type centers in halides, oxides and perovskites, Nucl. Instrum. Meth. Phys. Res. B 268 (2010) 3084.

[109] S. Kuck, I. Sokolska, Spectroscopic investigation of $\mathrm{Mn}^{2+}, \mathrm{Pr}^{3+}$ codoped $\mathrm{KMgF}_{3}$ under vacuum-ultraviolet excitation, J. Phys.: Condens. Matter 18 (2006) 54475457.

[110] P. Dorenbos, Lanthanide charge transfer energies and related luminescence, charge carrier trapping, and redox phenomena, J. Alloys Compd. 488 (2009) 568573. 
[111] W. Shuen, Z. Li, Algebraic method of determining bond length of perovskite compounds doped with $\mathrm{Mn}^{2+}$, Physica B 270 (1999) 244-248.

[112] H. Qiao, T. Liu, Q. Zhang, F. Cheng, X. Zhou, Study of the electronic structures of oxygen doped in $\mathrm{LiBaF}_{3}$ crystal, Nucl. Instrum. Meth. Phys. Res. B 267 (2009) $2467-2470$.

[113] N. Shiran, V. Voronova, Luminescence of oxygen and magnesium containing $\mathrm{LiBaF}_{3}$ crystals, J. Lumin 87-89 (2000) 561-563.

[114] L. Qi, M. Guo, L. Han, H. Gu, Electrolytic coloration and spectral properties of $\mathrm{O}^{2-}$ and $\mathrm{Mg}^{2+}$-codoped LiF crystals, Spectrochim. Acta A 152 (2016) 438-442.

[115] A. V. Gektin, I. M. Krasovitskaya, N. V. Shiran, UV-dosimetric material based on $\mathrm{KMgF}_{3}$ perovskite, Radiat. Meas. 29 (1998) 337-340.

[116] A. V. Gektin, I. M. Krasovitskaya, N. V. Shiran, High-temperature thermoluminescence of $\mathrm{KMgF}_{3}$-based crystals, Radiat. Meas. 72-74 (1997) 664-666.

[117] E. Radzhabov, P. Figura, Optical properties of oxygen-vacancy centers in fluorite, Phys. Stat. Sol. B 136 (1986) K55.

[118] E. Radzhabov, P. Figura, Optical properties of oxygen-vacancy defects in alkalineearth fluoride crystals, Phys. Stat. Sol. B 186 (1994) K37.

[119] E. Radzhabov, Time-resolved luminescence of oxygen-vacancy centres in alkalineearth fluoride and barium fluorohalide crystals, J. Phys.: Condens. Matter 6 (1994) 9807-9815.

[120] E. Radzhabov, V. Otroshok, Optical spectra of oxygen defects in $\mathrm{BaFCl}$ and $\mathrm{BaFBr}$ crystals, J. Phys. Chem. Solids 56 (1995) 1-7.

[121] A. Dauletbekova, L. Lisitsyna, V. Korepanov, V. Lisitsyn, L. Trefilova, R. Kassymkanova, Radiation transformation of the oxygen-containing impurity in LiF crystals doped with different polyvalent cations, Phys. Stat. Sol. C 10 (2013) 263-267.

[122] D. K. Sardar, M. D. Shinn, W. A. Sibley, Radiation-defect-perturbed $\mathrm{Er}^{3+}$ and $\mathrm{Mn}^{2+}$ optical transitions in $\mathrm{RbMgF}_{3}$, Phys. Rev. B 26 (1982) 2382-2389. 
[123] W. A. Sibley, N. Koumvakalis, Perturbed $\mathrm{Mn}^{2+}$ transitions in irradiated $\mathrm{RbMgF}_{3}: \mathrm{Mn}$, Phys. Rev. B 14 (1976) 35-40.

[124] D. K. Sardar, Electron irradiation damage in pure and impurity-doped $\mathrm{RbMgF}_{3}$ crystals, Phys. Stat. Sol. B 171 (1992) 39-50.

[125] S. I. Yun, K. H. Lee, W. A. Sibley, W. E. Vehse, Use of 3d-impurity-ion absorption to study the distribution of radiation damage in crystals, Phys. Rev. B 10 (1974) $1665-1672$.

[126] K. H. Lee, W. A. Sibley, Exchange enhancement of $\mathrm{Co}^{2+}$ and $\mathrm{Mn}^{2+}$ transitions due to radiation defects, Phys. Rev. B 12 (1975) 3392-3399.

[127] S. I. Yun, L. A. Kappers, W. A. Sibley, Enhancement of impurity-ion absorption due to radiation-produced defects, Phys. Rev. B (1973) 773-779.

[128] S. W. S. McKeever, B. Jassemnejad, J. Landreth, Manganese absorption in $\mathrm{CaF}_{2}: \mathrm{Mn}$; I, J. Appl. Phys. 60 (1986) 1124.

[129] A. C. Lewandowski, T. M. Wilson, Lattice-embedded multiconfigurational selfconsistent-field calculations of the Mn-perturbed F-center defect in $\mathrm{CaF}_{2}: \mathrm{Mn}$, Phys. Rev. B 52 (1995) 100-109.

[130] J. R. Seretlo, J. J. Martin, E. Sonder, Optical absorption of radiation-produced defects in $\mathrm{NaMgF}_{3}$ and $\mathrm{RbCaF}_{3}$, Phys. Rev. B 14 (12) (1976) 5404-5412.

[131] F. Cheng, T. Liu, Q. Zhang, H. Qiao, X. Zhou, Computer simulation of the electronic structures and absorption spectra for a $\mathrm{KMgF}_{3}$ crystal containing a potassium vacancy, Chin. Phys. Lett. 28 (2011) 036106.

[132] N. Kristianpoller, B. Trieman, Defects induced in $\mathrm{NaMgF}_{3}$ crystals by X- and VUVirradiation, J. Phys. Colloques 41 (1980) 109-111.

[133] J. J. Schuyt, J. Donaldson, G. V. M. Williams, S. V. Chong, Modelling the radioluminescence of $\mathrm{Sm}^{2+}$ and $\mathrm{Sm}^{3+}$ in the dosimeter material $\mathrm{NaMgF}_{3}: \mathrm{Sm}$, J. Phys. Condens. Matt. 32 (2020) 025703.

[134] E. G. Yukihara, V. H. Whitley, S. W. S. McKeever, A. E. Akselrod, M. S. Akselrod, Effect of high-dose irradiation on the optically stimulated luminescence of $\mathrm{Al}_{2} \mathrm{O}_{3}: \mathrm{C}$, Radiat. Meas. 38 (2004) 317. 
[135] C. G. Ma, M. G. Brik, Hybrid density-functional calculations of structural, elastic and electronic properties for a series of cubic perovskites $\mathrm{CsMF}_{3}(\mathrm{M}=\mathrm{Ca}, \mathrm{Cd}, \mathrm{Hg}$, and $\mathrm{Pb}$ ), Comp. Mater. Sci. 58 (2012) 101-112.

[136] A. J. J. Bos, Theory of thermoluminescence, Radiat. Meas. 41 (2007) S45-S56.

[137] V. Pagonis, G. Kitis, Prevalence of first-order kinetics in thermoluminescence materials: An explanation based on multiple competition processes, Phys. Stat. Sol. B 249 (2012) 1590-1601.

[138] M. S. Akselrod, S. S. Orlov, G. M. Akselrod, Bit-Wise Volumetric Optical Memory Utilizing Two-Photon Absorption in Aluminium Oxide Medium, Jpn. J. Appl. Phys. 43 (2004) 4908-4911.

[139] R. H. J. Fastenau, E. J. van Loenen, Applications of rare earth permanent magnets, J. Magnetism Magnetic Mater. 157/158 (1996) 1-6.

[140] H. Riesen, W. A. Kaczmarek, Efficient X-ray Generation of $\mathrm{Sm}^{2+}$ in Nanocrystalline $\mathrm{BaFCl} / \mathrm{Sm}^{3+}$ : a Photoluminescent X-ray Storage Phosphor, Inorg. Chem. 46 (2007) 7235-7237.

[141] G. Okada, S. Vahedi, B. Morrell, C. Koughia, G. Belev, T. Wysokinsk, D. Chapman, C. Varoy, A. Edgar, S. Kasap, Examination of the dynamic range of Sm-doped glasses for high-dose and high-resolution dosimetric applications in microbeam radiation therapy at the Canadian synchrotron, Opt. Mater. 35 (2013) 1976-1980.

[142] N. Chiodini, A. Vedda, I. Veronese, Rare earth doped silica optical fibre sensors for dosimetry in medical and technical applications, Adv. Opt. 2014 (2014) 1-9.

[143] V. S. K. Kumar, B. S. V. S. R. Acharyulu, S. B. S. Sastry, Redox reaction and thermoluminescence in dysprosium-doped potassium sulphate phosphor, J. Lumin. 55 (1993) 42-48.

[144] I. Gerard, J. C. Krupa, E. Simoni, P. Martin, Investigation of charge transfer $\mathrm{O}^{2-}$ - $\mathrm{Ln}^{3+}$ and $\mathrm{F}^{-}-\mathrm{Ln}^{3+}$ in $\mathrm{LaF}_{3}:\left(\mathrm{Ln}^{3+}, \mathrm{O}^{2-}\right)$ and $\mathrm{YF}_{3}\left(\mathrm{Ln}^{3+}, \mathrm{O}^{2-}\right)$ systems, J. Alloys Compd. 207/208 (1994) 120-127.

[145] P. Dorenbos, Charge transfer bands in optical materials and related defect level location, Opt. Mater. 69 (2017) 8-22. 
[146] G. Wakefield, E. Holland, P. J. Dobson, J. L. Hutchison, Luminescence properties of nanocrystalline $\mathrm{Y}_{2} \mathrm{O}_{3}: \mathrm{Eu}, \mathrm{Adv}$. Mater. 13 (2001) 1557-1560.

[147] X. Huang, S. Han, W. Huang, X. Liu, Enhancing solar cell efficiency: the search for luminescent materials as spectral converters, Chem. Soc. Rev. 42 (2013) 173-201.

[148] P. Dorenbos, Systematic behaviour in trivalent lanthanide charge transfer energies, J. Phys.: Condens. Matter 15 (2003) 8417-8434.

[149] P. Dorenbos, Energy of the $\mathrm{Eu}^{2+} 5 \mathrm{~d}$ state relative to the conduction band in compounds, J. Lumin. 128 (2008) 578-582.

[150] P. Dorenbos, Modeling the chemical shift of lanthanide $4 \mathrm{f}$ electron binding energies, Phys. Rev. B 85 (2012) 165107.

[151] B. Morell, G. Okada, S. Vahedi, C. Koughia, A. Edgar, C. Varoy, G. Belev, T. Wysokinski, R. Sammynaiken, S. O. Kasap, Optically erasable samarium-doped fluorophosphate glasses for high-dose measurements in microbeam radiation therapy, J. Appl. Phys. 115 (2014) 063107.

[152] J. Qiu, K. Miura, T. Suzuki, T. Mitsuyu, K. Hirao, Permanent photoreduction of $\mathrm{Sm}^{3+}$ to $\mathrm{Sm}^{2+}$ inside a sodium aluminoborate glass by an infrared femtosecond pulsed laser, Appl. Phys. Lett. 74 (1999) 10-12.

[153] A. Z. M. S. Rahman, X. Cao, L. Wei, B. Wang, R. Yu, Z. Chen, G. An, A. Sidike, Irradiation-induced valence conversion of samarium ions in $\mathrm{Na}_{2} \mathrm{SO}_{4}$, Appl. Phys. A 111 (2013) 587-591.

[154] K. Binnemans, Interpretation of europium(III) spectra, Coord. Chem. Rev. 295 (2015) 1-45.

[155] X. Y. Chen, G. K. Liu, The standard and anomalous crystal-field spectra of $\mathrm{Eu}^{3+}$, J. Solid State Chem. 178 (2005) 419-428.

[156] D. C. Yu, X. Y. Huang, S. Ye, Q. Y. Zhang, J. Wang, A sequential two-step nearinfrared quantum splitting in $\mathrm{Ho}^{3+}$ singly doped $\mathrm{NaYF}_{4}$, AIP Advances 1 (2011) 042161.

[157] M. Malinowski, Z. Fukacz, M. Szuflinska, A. Wnuk, M. Kaczkan, Optical transitions of $\mathrm{Ho}^{3+}$ in YAG, J. Alloys Compd. 300-301 (2000) 389-394. 
[158] R. B. Hughes-Currie, P. S. Senanayake, J. R. Wells, M. F. Reid, G. Berden, R. J. Reeves, A. Meijerink, Site-selective transient photoluminescence enhancement of impurity-trapped excitons in NaMgF3:Yb ${ }^{2+}$, Phys. Rev. B 88 (2013) 104304.

[159] R. B. Hughes-Currie, K. V. Ivanovskikh, J. R. Wells, M. F. Reid, A. Meijerink, Intrinsic electronic excitations and impurity luminescent centres in $\mathrm{NaMgF}_{3}$ and $\mathrm{MgF}_{2}$ doped with $\mathrm{Yb}^{2+}$, Opt. Mater. 99 (2019) 109553.

[160] R. B. Hughes-Currie, K. V. Ivanovskikh, J. P. R. Wells, M. F. Reid, R. A. Gordon, The determination of dopant ion valence distributions in insulating crystals using XANES measurements, J. Phys.: Condens. Matter 28 (2016) 135502.

[161] N. Guerassimova, N. Garnier, C. Dujardin, A. G. Petrosyan, C. Pedrini, X-ray excited charge transfer luminescence of ytterbium-containing aluminium garnets, Chem. Phys. Lett. 339 (2001) 197-202.

[162] R. K. Sharma, A.-V. Mudring, P. Ghosh, Recent trends in binary and ternary rareearth fluoride nanophosphors: how structural and physical properties influence optical behavior, J. Lumin. 189 (2017) 44-63.

[163] J. H. V. Vleck, The theory of electric and magnetic susceptibilities, Oxford University Press, Oxford, United Kingdom, 1932.

[164] K. K. Rao, M. Vithal, D. Ravinder, Preparation, infrared and magnetic susceptibility studies of $\mathrm{LnB}_{3} \mathrm{O}_{6}(\mathrm{Ln}=\mathrm{Gd}, \mathrm{Eu}$, and Sm), J. Magnetism Magnetic Mater. 253 (2002) 65-71.

[165] G. A. Bain, J. F. Berry, Diamagnetic corrections and pascal's constants, J. Chem. Education 85 (2008) 532-536.

[166] S. Vahedi, G. Okada, B. Morell, E. Muzar, C. Koughia, A. Edgar, C. Varoy, G. Belev, T. Wysokinski, D. Chapman, S. Kasap, X-ray induced $\mathrm{Sm}^{3+}$ to $\mathrm{Sm}^{2+}$ conversion in fluorophosphate and fluoroaluminate glasses for the monitoring of high-doses in microbeam radiation therapy, J. Appl. Phys. 112 (2012) 073108.

[167] R. Chen, P. L. Leung, The decay of OSL signals as stretched-exponential functions, Radiat. Meas. 37 (2003) 519.

[168] A. F. van Driel, I. S. Nikolaev, P. Vergeer, P. Lodahl, D. Vanmaekelbergh, W. L. Vos, Statistical analysis of time-resolved emission from ensembles of semiconductor 
quantum dots: Interpretation of exponential decay models, Phys. Rev. B 75 (2007) 035329.

[169] P. Dorenbos, Thermal quenching of $\mathrm{Eu}^{2+} 5 \mathrm{~d}-4 \mathrm{f}$ luminscence in inorganic compounds, J. Phys.: Condens. Matter 17 (2005) 8103-8111.

[170] T. M. Tolhurst, S. Schmiechen, P. Pust, P. J. Schmidt, W. Schnick, A. Moewes, Electronic structure, bandgap, and thermal quenching of $\mathrm{Sr}\left[\mathrm{Mg}_{3} \mathrm{SiN}_{4}\right]: \mathrm{Eu}^{2+}$ in comparison to $\mathrm{Sr}\left[\mathrm{LiAl}_{3} \mathrm{~N}_{4}\right]: \mathrm{Eu}^{2+}$, Adv. Opt. Mater. 4 (2016) 584-591.

[171] U. Happek, S. A. Basun, J. Choi, J. K. Krebs, M. Raukas, Electron transfer processes in rare earth doped insulators, J. Alloys Compd. 303-304 (2000) 198-206.

[172] N. R. J. Poolton, A. J. J. Bos, P. Dorenbos, Luminescence emission from metastable $\mathrm{Sm}^{2+}$ defects in $\mathrm{YPO}_{4}: \mathrm{Ce}, \mathrm{Sm}, \mathrm{J}$. Phys.: Condens. Matter 24 (2012) 225502.

[173] A. Mainwood, Recent developments of diamond detectors for particles and UV radiation, Semicond. Sci. Technol. 15 (2000) R55-R63.

[174] W. Pan, H. Wu, J. Luo, Z. Deng, C. Ge, C. Chen, X. Jiang, W. Yin, G. Niu, L. Zhu, L. Yin, Y. Zhou, Q. Xie, X. Ke, M. Sui, J. Tang, $\mathrm{Cs}_{2} \mathrm{AgBiBr}_{6}$ single-crystal X-ray detectors with a low detection limit, Nature Photonics 11 (2017) 726-734.

[175] R. S. Lee, Radiation induced currents across $\mathrm{Al}_{2} \mathrm{O}_{3}$ films, IEEE Transactions on Nuclear Science 17 (1970) 47-51.

[176] B. Nagaraj, S. Aggarwal, R. Ramesh, Influence of contact electrodes on leakage characteristics in ferroelectric thin films, J. Appl. Phys. 90 (2001) 375-382.

[177] R. W. Klaffky, B. H. Rose, A. N. Goland, G. J. Dienes, Radiation-induced conductivity of $\mathrm{Al}_{2} \mathrm{O}_{3}$ : Experiment and theory, Phys. Rev. B 21 (1980) 3610-3634.

[178] Y. He, L. Matei, H. J. Jung, K. M. McCall, M. Chen, C. C. Stoumpos, Z. Liu, J. A. Peters, D. Y. Chung, B. W. Wessels, M. R. Wasielewski, V. P. Dravid, A. Burger, M. G. Kanatzidis, High spectral resolution of gamma-rays at room temperature by perovskite $\mathrm{CsPbBr}_{3}$ single crystals, Nature Communications 9 (2018) 1609.

[179] S. Yakunin, D. N. Dirin, Y. Shynkarenko, V. Morad, I. Cherniukh, O. Nazarenko, D. Kreil, T. Nauser, M. V. Kovalenko, Detection of gamma photons using solutiongrown single crystals of hybrid lead halide perovskites, Nature Photonics 10 (2016) 585-590. 
[180] G. Conte, M. C. Rossi, S. Salvatori, P. Ascarelli, D. Trucchi, Thin polycrystalline diamond for low-energy x-ray detection, J. Appl. Phys. 96 (2004) 6415-6420.

[181] H. Wei, D. DeSantis, W. Wei, Y. Deng, D. Guo, T. J. Savenije, L. Cao, J. Huang, Dopant compensation in alloyed $\mathrm{CH}_{3} \mathrm{NH}_{3} \mathrm{PbBr}_{3-x} \mathrm{Cl}_{x}$ perovskite single crystals for gamma-ray spectroscopy, Nature Materials 16 (2017) 826-834.

[182] H. Wei, Y. Fang, P. Mulligan, W. Chuirazzi, H. Fang, C. Wang, B. R. Ecker, Y. Gao, M. A. Loi, L. Cao, J. Huang, Sensitive X-ray detectors made of methylammonium lead tribromide perovskite single crystals, Nature Photonics 10 (2016) 333-340.

[183] H. Wei, J. Huang, Halide lead perovskites for ionizing radiation detection, Nature Communications 10 (2019) 1066.

[184] T. Wulz, W. Gerding, N. lavrik, D. Briggs, B. Srijanto, K. Lester, D. Hensley, S. Spanier, E. Lukosi, Realization of deep 3D metal electrodes in diamond radiation detectors, Appl. Phys. Lett. 112 (2018) 222101.

[185] J. Isberg, J. Hammersberg, H. Bernhoff, D. J. Twitchen, A. J. Whitehead, Charge collection distance measurements in single and polycrystalline CVD diamond, Diamond and Related Materials 13 (2004) 872-875.

[186] C. Dotzler, G. V. M. Williams, U. Rieser, J. Robinson, Photoluminescence, optically stimulated luminescence, and thermoluminescence study of $\mathrm{RbMgF}_{3}: \mathrm{Eu}^{2+}$, J. Appl. Phys. 105 (2009) 023107.

[187] O. Gallot-lavallee, G. Teyssedre, C. Laurent, S. Rowe, Identification of photoluminescence features of an epoxy resin based on components features and curing effects, Polymer 46 (2005) 2722-2731.

[188] V. Griseri, L. A. Dissado, J. C. Fothergill, C. Laurent, G. Teyssedre, Photoluminescence, recombination induced luminescence and electroluminescence in epoxy resin, J. Phys. D: Appl. Phys. 34 (2001) 2534-2540.

[189] A. Edgar, G. V. M. Williams, J. Hamlin, M. Secu, S. Schweizer, J. M. Spaeth, New materials for glass-ceramic X-ray storage phosphors, Current Appl. Phys. 4 (2004) 193-196.

[190] C. G. Soares, Radiochromic film dosimetry, Radiat. Meas. 41 (2007) S100-S116. 
[191] S. Devic, Radiochromic film dosimetry: past, present and future, Physica Medica 27 (2011) 122-134.

[192] G. V. M. Williams, A. Bittar, C. Dotzler, A. Beaudin, C. Varoy, C. Dunford, Glassceramics and epoxy-composites for radiation imaging, Radiat. Meas. 42 (2007) 899-902. 


\section{Appendix A}

\section{List of Publications}

The following publications were produced during the course of this research.

J. J. Schuyt and G. V. M. Williams, The effect of ionizing radiation on the optical properties of $\mathrm{NaMgF}_{3}(\mathrm{Mn})$ : Observation of an F-center Mn complex, Journal of Applied Physics 122, 063107 (2017).

J. J. Schuyt and G. V. M. Williams, Radiation-induced changes in the optical properties of $\mathrm{NaMgF}_{3}(\mathrm{Sm})$ : Observation of resettable Sm radio-photoluminescence, Materials Research Bulletin 106, 455-458 (2018).

G. V. M. Williams, J. J. Schuyt, A. S. Madathiparambil, The effect of Mn concentration on the luminescence properties of $\mathrm{NaMgF}_{3}: \mathrm{Mn}$ : Defect/Mn complex photoluminescence, radioluminescence, and optically stimulated luminescence for radiation dose monitoring, Optical Materials 84, 763-770 (2018).

J. J. Schuyt and G. V. M. Williams, Photoluminescence, radioluminescence and optically stimulated luminescence in nanoparticle and bulk $\mathrm{KMgF}_{3}(\mathrm{Eu})$, Journal of Luminescence 204, 472-479 (2018).

J. J. Schuyt and G. V. M. Williams, $2 D$ dosimetry using the optically stimulated luminescence of $\mathrm{NaMgF}_{3}:$ Eu with CCD camera readout, Radiation Measurements 121, 99-102 (2019).

J. J. Schuyt and G. V. M. Williams, F-centre/Mn complex photoluminescence in the fluoroperovskites $A M g F_{3}: M n(A=N a, K$, or $R b)$, Optical Materials: X 1, 100010, (2019). 
J. J. Schuyt and G. V. M. Williams, Oxygen-impurity charge transfer in $\mathrm{NaMgF}_{3}: \mathrm{Ln}$ $(\mathrm{Ln}=\mathrm{Yb}, \mathrm{Sm}$, or Eu): Establishing the lanthanide energy levels in $\mathrm{NaMgF}$, Journal of Luminescence 211, 413-417 (2019).

J. J. Schuyt and G. V. M. Williams, Optical properties of $\mathrm{Mn}^{2+}$ doped $C s C d F_{3}$ : A potential real-time and retrospective $U V$ and $X$-ray dosimeter material, Journal of Applied Physics 125, 233102 (2019).

J. J. Schuyt and G. V. M. Williams, Quenching of the $\mathrm{Sm}^{2+}$ luminescence in $\mathrm{NaMgF}_{3}: \mathrm{Sm}$ via photo-thermal ionization: Alternative method to determine divalent lanthanide trap depths, Applied Physics Letters 115, 181104 (2019).

J. J. Schuyt, J. Donaldson, G. V. M. Williams, S. V. Chong, Modelling the radioluminescence of $\mathrm{Sm}^{2+}$ and $\mathrm{Sm}^{3+}$ in the dosimeter material $\mathrm{NaMgF}_{3}: \mathrm{Sm}$, Journal of Physics: Condensed Matter 32, 025703 (2020).

J. J. Schuyt and G. V. M. Williams, Photoluminescence of $\mathrm{Dy}^{3+}$ and $\mathrm{Dy}^{2+}$ in $\mathrm{NaMgF}_{3}: \mathrm{Dy}$ : A potential infrared radiophotoluminescence dosimeter, Radiation Measurements 134, 106326 (2020). 